\title{
Motivation von \\ Seniorinnen und Senioren \\ zur sportlichen Betätigung: \\ Eine empirische Untersuchung \\ mittels qualitativer Interviews \\ in und um Göttingen
}

\author{
Dissertation \\ zur Erlangung des Doktorgrades \\ der Sozialwissenschaftlichen Fakultät \\ der Georg-August-Universität Göttingen
}

vorgelegt von

Rita Spiller

geboren in Bremen

Göttingen 2015 
Erstgutachter: Prof. Dr. Arnd Krüger

Zweitgutachter: Dr. Arne Göring

Drittgutachterin: Prof. Dr. llona Ostner

Tag der mündlichen Prüfung: 30.06.2015 


\section{Inhaltsverzeichnis}

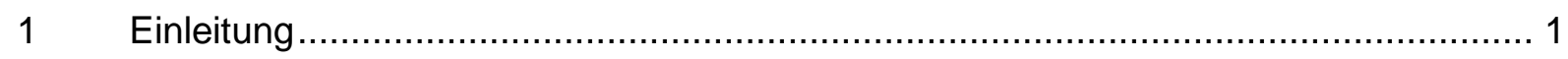

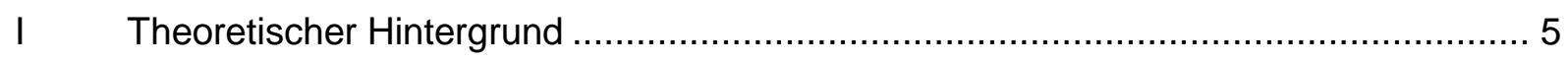

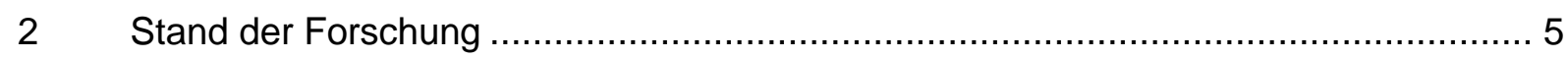

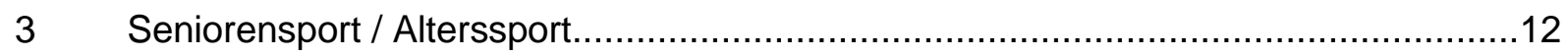

3.1 Begriffsbestimmung ,Seniorenalter' ............................................................ 12

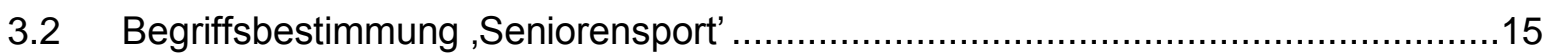

3.3 Der Begriff der Salutogenese nach Antonovsky (1997) ….................................20

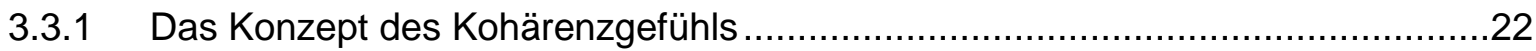

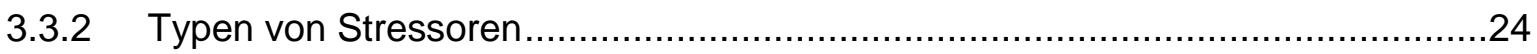

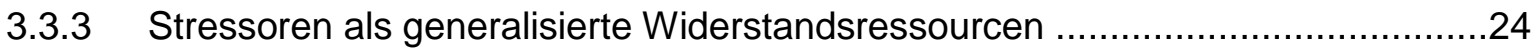

3.3.4 Kontrollstudien zu Antonovskys Theorie der Salutogenese ..............................26

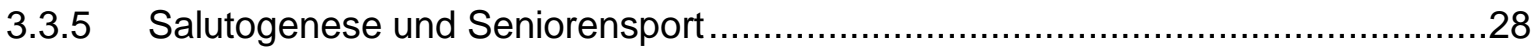

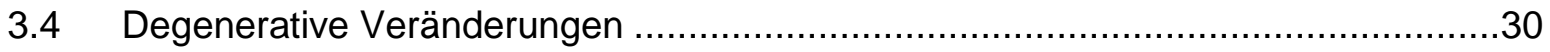

3.5 Konditionelle Fähigkeiten - Trainierbarkeit und Bedingungen im Alter ..................32

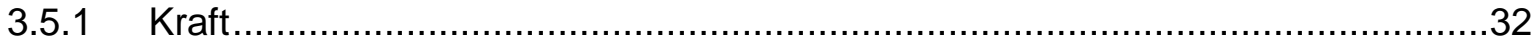

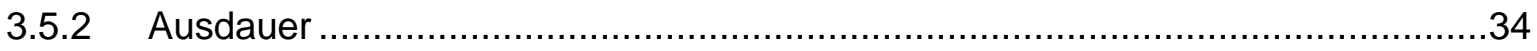

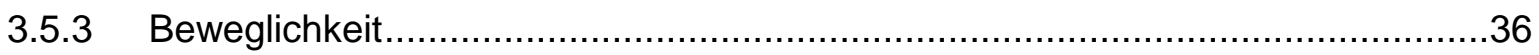

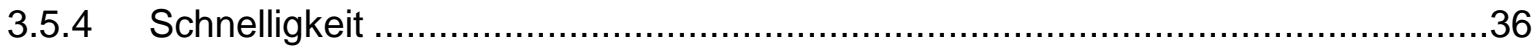

3.6 Koordinative Fähigkeiten - Trainierbarkeit und Bedingungen im Alter ...................37

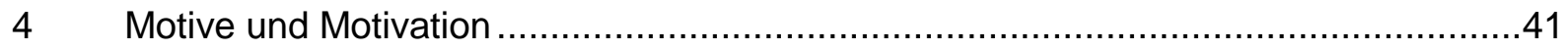

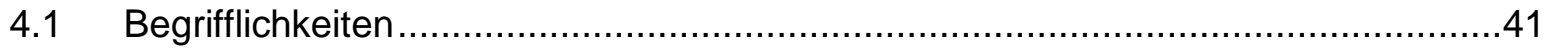

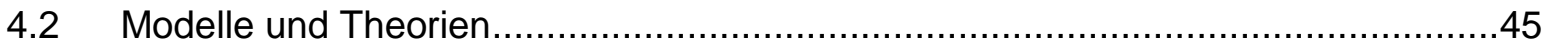

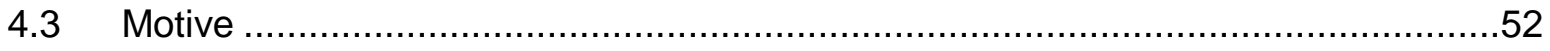

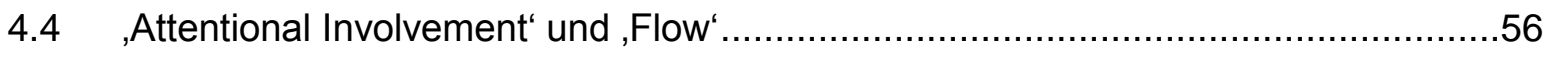

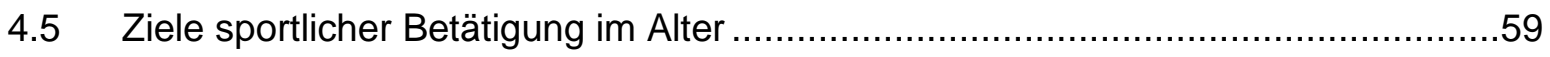

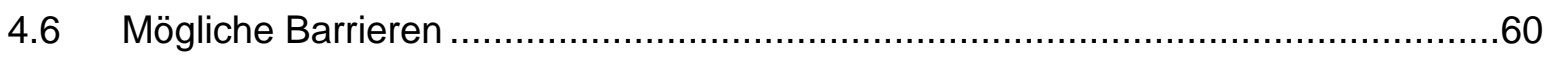

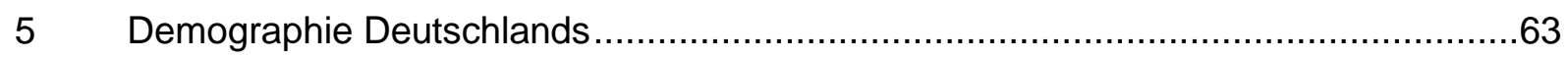

5.1 Bevölkerungsentwicklung in Deutschland...................................................63

5.2 Voraussichtliche Konsequenzen für die Entwicklung des Seniorensports...............73

5.3 Altersstatistiken des Deutschen Olympischen Sportbundes (DOSB) ......................74

5.4 Förderungsprogramme für Seniorensport des Deutschen Olympischen Sportbundes

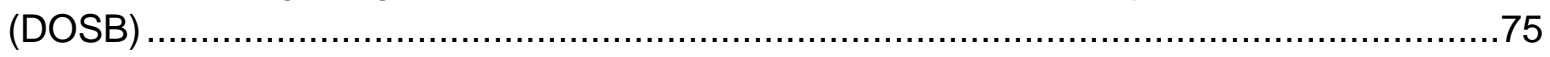

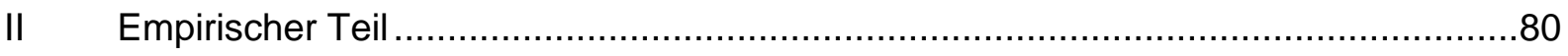

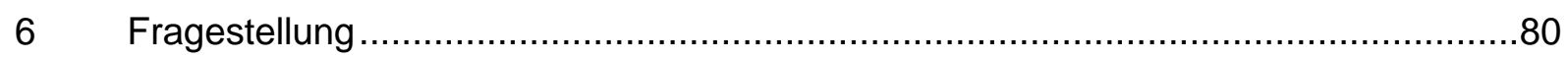

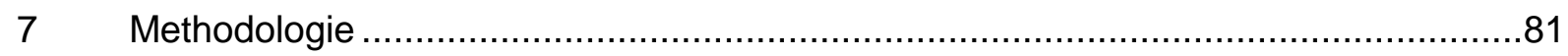




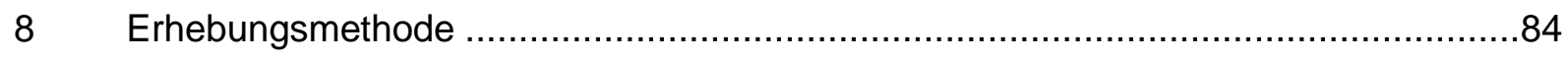

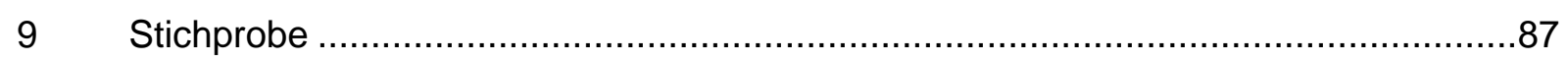

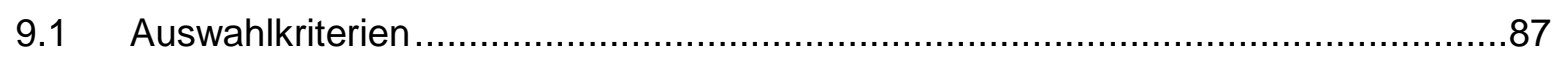

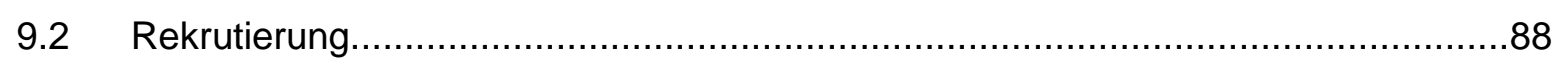

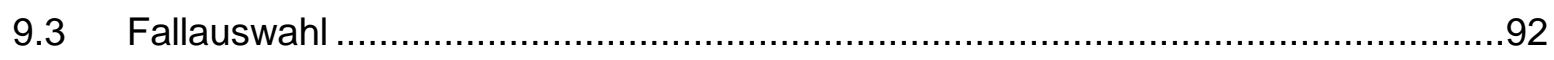

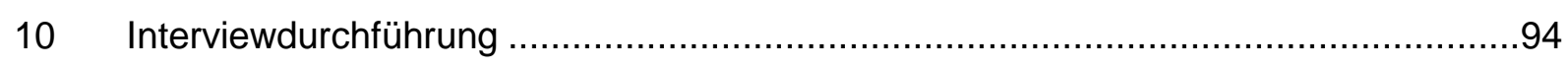

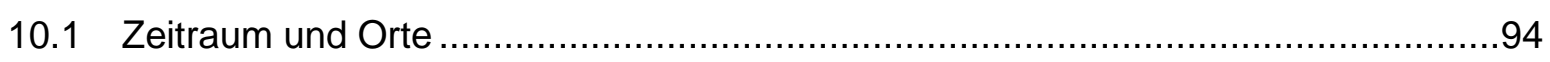

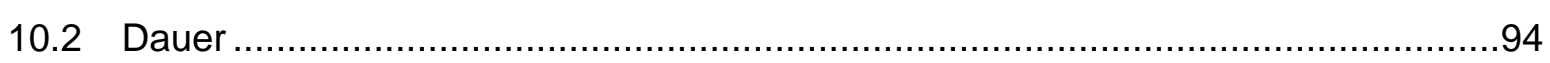

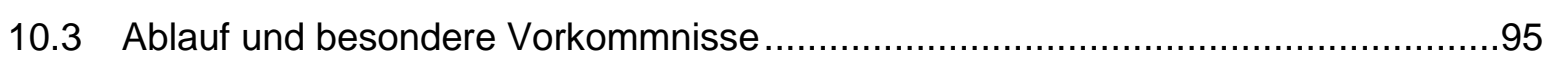

10.4 Ablauf und Begründung des Migrantinnen-Interviews.....................................97

10.5 Teilnahmebereitschaft der Befragten.........................................................

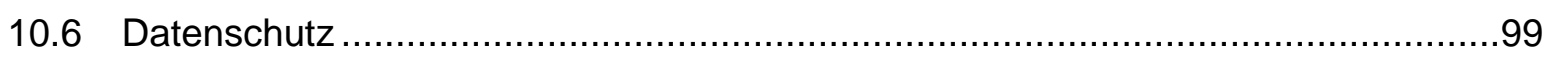

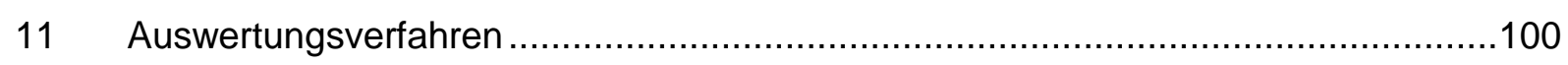

11.1 Qualitative Inhaltsanalyse nach Mayring .................................................. 100

11.2 Beispiel der Auswertung mithilfe der qualitativen Inhaltsanalyse .......................105

11.3 Texthermeneutische Auswertungsverfahren.................................................107

11.4 Beispiel der Auswertung mithilfe eines hermeneutischen Verfahrens ..................109

11.5 Kritische Reflexion................................................................................... 111

12 Ergebnisse der Interviewauswertungen ..................................................... 113

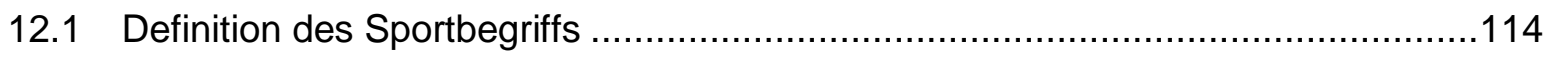

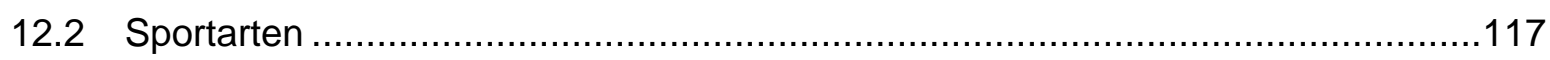

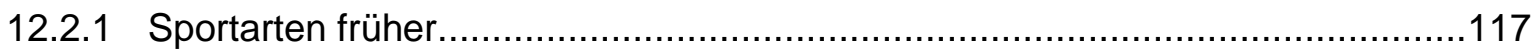

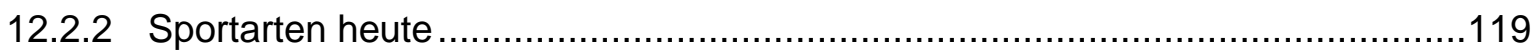

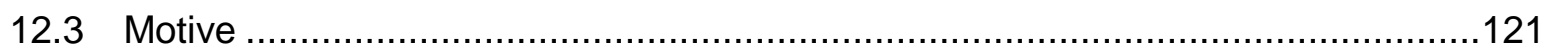

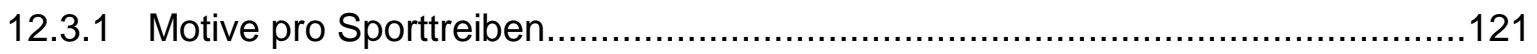

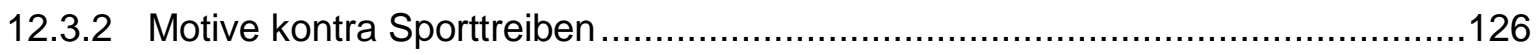

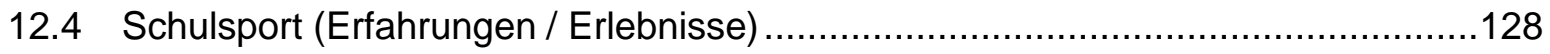

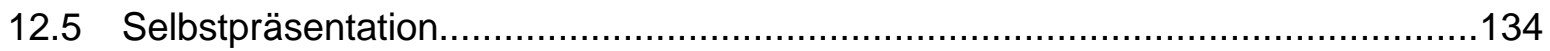

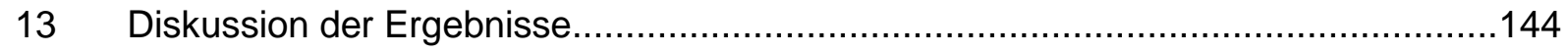

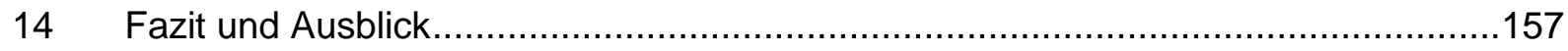

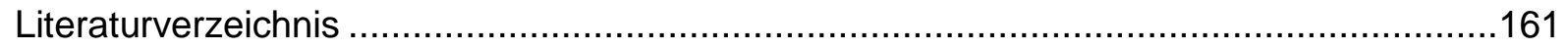

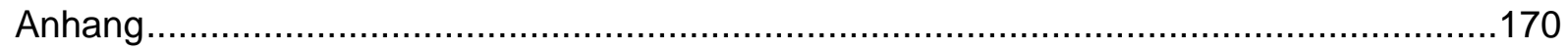

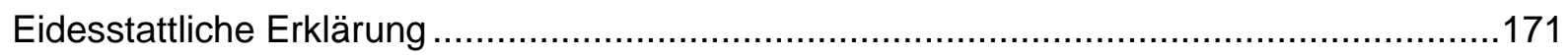




\section{Abbildungsverzeichnis}

Abbildung 1: Schaubild zur Erläuterung der GRRs und GRDs (Rita Spiller)

Abbildung 2: Rubikon-Modell nach Heckhausen 1989 (Sudeck 2007: 46)

48

Abbildung 3: Bevölkerung nach Altersgruppen 2008 und 2030 (Variante der

Vorausberechnung mit der mittleren Bevölkerung von drei möglichen Varianten) in \% der

Gesamtbevölkerung (Statistisches Bundesamt 2011: 25).

Abbildung 4: Lebenserwartung ab 60 Jahre für Männer und Frauen, 1871 bis 2060

(Statistisches Bundesamt 2011)

Abbildung 5: Wanderungssaldo 1954 bis 2060 (Statistisches Bundesamt 2011)

Abbildung 6: Altersaufbau der Bevölkerung in Deutschland (Statistisches Bundesamt 2011).

Abbildung 7: Pflegequoten 2005 (Statistisches Bundesamt 2011)

Abbildung 8: Altersverteilung der Stichprobe.....

Abbildung 9: Ausgeübte Sporttätigkeiten

Abbildung 10: Interviewdauer .95 



\section{$1 \quad$ Einleitung}

In unserer heutigen Gesellschaft liegt - aufgrund des demographischen Wandels - der Fokus auf den Seniorinnen und Senioren (vgl. Statistisches Bundesamt 2012). Deshalb ist die intensive Betrachtung dieser Zielgruppe unabdingbar geworden. Insbesondere Faktoren, wie ein idealerweise langer und unabhängiger, möglichst gesunder Lebensabend und die hiermit verbundenen geringen Betreuungskosten, spielen in der aktuellen Zeit eine große Rolle. Hier liegt es daher nahe, Möglichkeiten hervorzuheben, welche eine langfristigere Unabhängigkeit im Alter evozieren können. Dass Sport ein solches Mittel sein kann und sogar sein sollte, ist heutzutage längst nicht mehr umstritten. Lediglich die Intensität und Art des Sporttreibens wird, insbesondere für den Seniorinnen- und Seniorenbereich, noch diskutiert.

Die meisten Studien über Seniorensport befassen sich mit dem Leistungssport bzw. Wettkampfsport, also mit den physisch Aktiven, welche im hohen Alter immer noch an Wettkämpfen und Leistungsvergleichen teilnehmen (vgl. z.B. Keller 2015; Leigey et al. 2009; Feland, Hager \& Merrill 2005). Hierbei wird unterschieden zwischen Seniorinnen und Senioren, die bereits in ihrer Biographie lebenslanges Sporttreiben vorweisen können und jenen, die erst im fortgeschrittenen Alter mit dem Leistungssport beginnen. Außerdem beschäftigen sich viele Studien, die den Seniorensport thematisieren, mit physischen Veränderungen, das heißt körperlichen Adaptationen, welche resultierend aus dem Sporttreiben entstehen können (vgl. hierzu z.B. Willimczik, K. \& Singer, R. 2009; Dean, R.N. et al. 2007). Darüber hinaus existieren Studien über Breitensport meist aus quantitativer Forschungssicht, wobei Daten mithilfe von Fragebögen bezüglich der Sportpartizipation der Seniorinnen und Senioren ausgewertet werden (vgl. z.B. Guay, Vallerand \& Blanchard 2000; Brand 2006).

Analog zu diesen beschriebenen Studien soll diese Dissertation zunächst zum einen das Phänomen der Motivation von Seniorinnen und Senioren zur sportlichen Betätigung aufzeigen. Hierbei handelt es sich bei allen Fällen um 
Freizeitsport, da die Gründe nicht so offensichtlich erscheinen wie im Leistungssport (z.B. Ruhm, Preisgeld, Berühmtheit): „Exploring motivation in the context of leisure seems to be the ideal way of understanding why people engage or not in an activity and whether they are avid or occasional participants" (Lapointe \& Perreault 2013: 136).

Obwohl es sicher eine Vielzahl an Gründen für ein Nichtsportlerleben gibt, ist ein Mangel an Motivation oftmals ein häufiger Faktor für diese Inaktivität (vgl. Hepler, Wang \& Albarracin 2012). Somit lautet die Hauptfragestellung dieser Arbeit:

Wie kommt es, dass manche Menschen im hohen Alter mit dem Sporttreiben beginnen, während andere inaktiv bleiben?

Des Weiteren werden folgende Fragen untersucht und beleuchtet: Was bewegt diese Menschen zu einer Veränderung ihrer bisher geführten inaktiven Lebensweise? Welche Motive entstehen und wie werden diese in die Tat umgesetzt? Warum geschieht diese Veränderung nur bei einer gewissen Anzahl von Personen und nicht grundsätzlich? Welche Art von Erlebnissen ist ausschlaggebend für das Sportverhalten einer Person? Diese Fragestellungen und noch viele andere sollen mit Hilfe von qualitativen (teilnarrativen) Interviews erforscht und analysiert werden.

Zum anderen erscheint eine empirische Untersuchung einer inaktiven Gruppe von hoher Bedeutung, um Vergleiche und Rückschlüsse ziehen zu können. Die Methode des Interviews verspricht eine hohe Anzahl an individuellen Interpretationsmöglichkeiten und ermöglicht eine spezielle Einsicht in die Psyche der Sportlerinnen und Sportler dank der direkten Interaktion von Angesicht zu Angesicht. Warum ist die Gruppe der inaktiven Seniorinnen und Senioren nicht motiviert, Sport zu treiben? Was unterscheidet sie von den gleichaltrigen Sportlerinnen und Sportlern? Wie könnte man dieser Gruppe von Nicht-Sportlern die sportliche Betätigung näherbringen? Gibt es „hoffnungslose Fälle"? Auch diese Fragestellungen sind Kern der qualitativen Untersuchung. 
In dieser Arbeit wird versucht, eine Differenzierung zu leisten zwischen Wiederanfängern, das heißt Seniorinnen und Senioren, die erst im Seniorenalter wieder mit dem Sporttreiben begannen, nachdem sie seit der Schulzeit inaktiv gewesen waren, und Nicht-Sportlern, also Seniorinnen und Senioren, welche seit dem Schulsport keine regelmäßigen sportlichen Aktivitäten mehr betrieben haben.

Darüber hinaus wäre es wünschenswert, eine Strategie zu entwickeln, welche auch die Nicht-Sportlerinnen und -Sportler zum Sporttreiben motiviert. Hier wird aufgezeigt, ob die Motive, die sich bei den Wiederanfängern herauskristallisiert haben, auch auf die Nicht-Sportlerinnen und -Sportler übertragbar wären.

Im theoretischen Teil dieser Arbeit geht es nach dem aktuellen Stand der Forschung um die Klärung von Begrifflichkeiten, wie etwa ,Seniorenalter', ,Seniorensport' oder ,Salutogenese', welche im Verlauf der Arbeit immer wieder eine der Kernrollen spielen und daher im Vorfeld klar definiert werden müssen. Das dritte Kapitel des Theorieteils handelt von degenerativen Veränderungen sowohl im koordinativen als auch im konditionellen Bereich, also um den altersbedingten Rückgang gewisser körperlicher Funktionen und seine Auswirkungen bzw. Möglichkeiten zur Prävention.

Im vierten Kapitel des Theorieteils folgt ein ausführlicher Überblick über die Konzepte und Theorien der Motivation inklusive einer Vorstellung von ausgewählten Studien zu dieser Thematik. Das Kapitel schließt mit Zielen sportlicher Betätigung im Alter sowie möglichen Barrieren der Sportausübung.

Das letzte große Kapitel des Theorieteils handelt von dem demographischen Wandel innerhalb Deutschlands. Hier wird aufgezeigt, inwieweit sich die Bevölkerung voraussichtlich während der nächsten Jahrzehnte verändern wird und welche Rolle eine gute gesundheitliche Verfassung, insbesondere im hohen Alter, spielt. Das Kapitel endet mit einer Vorstellung von ausgewählten Sportförderungsprogrammen für Seniorinnen und Senioren vom Deutschen Olympischen Sportbund (DOSB). 
Im anschließenden Empirieteil, dem praktischen Teil dieser Doktorarbeit, folgt zunächst eine theoretische Klärung der Methodologie und der Erhebungsinstrumente. Hier wird unter anderem begründet, warum eine qualitative Erhebungsmethode gewählt wurde im Vergleich zu einer möglichen quantitativen Forschungssystematik.

Diesen Kapiteln folgt die Beschreibung, Rekrutierung und genaue Fallzahl der interviewten Stichprobe. Dies beinhaltet zum einen die Kategorisierung der Interviewpartnerinnen und -partner in beispielsweise Individualsportlerinnen und -sportler, Fitnessstudiobesucherinnen und -besucher, Vereinsmitglieder oder Nicht-Sportlerinnen und -sportler. Zum anderen wird aufgezeigt, mit welchen Mitteln die Interviewpartnerinnen und -partner rekrutiert werden konnten und welche Probleme dabei auftraten.

Im Anschluss geht es um die Interviewdurchführung, also um die praktische Arbeit dieser Dissertation. Hier werden sowohl Zeitraum und Ort der Interviews, Dauer, besondere Vorkommnisse, Teilnahmebereitschaft der Befragten sowie die Thematik des Datenschutzes aufgegriffen. Hierauf folgt ein Kapitel bezüglich der Auswertungsverfahren, mit denen die qualitativen Interviews analysiert wurden. Auch hier wird eine Begründung für die gewählten Verfahren dargelegt. Nachdem konkrete Textstellen beispielhaft analysiert wurden, liegt das umfangreiche Kapitel der Ergebnisdarstellung vor.

Im Ergebniskapitel werden die Inhalte der Interviews nach Kategorien sortiert, welche innerhalb des Ergebniskapitels die einzelnen Unterkapitel bilden. Integriert sind in die Kapitel stets Zitate und Ausschnitte aus den unterschiedlichen Interviews zur besseren Verdeutlichung der Situationen und Gründe des Gesagten.

Im Anschluss folgen die Diskussion der Ergebnisse sowie das Fazit inklusive eines Ausblicks auf mögliche Folgestudien. 


\section{Theoretischer Hintergrund}

\section{$2 \quad$ Stand der Forschung}

Oftmals klingt die Motivation zum Sporttreiben bei Wiedereinsteigern nach einer Anfangseuphorie wieder ab. Vallerand et al. (2003) entwickelten ein Modell der Leidenschaft zum Sporttreiben, welches erklären soll, aus welchen Gründen manche Menschen regelmäßig Sport treiben und andere oftmals nur gelegentlich. In ihrer Studie schildern sie zwei Typen der Leidenschaft: zum einen die harmonische Leidenschaft, welche dem Individuum die gänzliche Kontrolle über die Betätigung lässt, zum anderen die zwanghafte Leidenschaft, welche einen derartigen Druck auf das Individuum ausübt, dass dieses die Kontrolle über den eigenen Willen verliert. Demzufolge sind zwanghafte Leidenschaften eher negativ konnotiert, während harmonische Leidenschaften eher positive Assoziationen hervorrufen. Sportliche Betätigung sollte also, um ein positives Wohlbefinden zu erhalten und zu fördern, stets mit harmonischer Leidenschaft behaftet sein und niemals zwanghaft ausarten (vgl. Lapointe \& Perreault 2013). Diese Studie präsentiert einen guten Ansatz, welcher bei der individuellen Sportauswahl von Nicht-Sportlerinnen und -Sportlern sowie Wiederanfängern eine wichtige Rolle spielt: Anbieter sollten immer das Ziel verfolgen, harmonische Leidenschaften hervorzurufen und dementsprechend positive Gefühle in Bezug zum Sporttreiben zu evozieren.

Um die einzelnen Komponenten der SDT (Self-Determination Theory, im weiteren Verlauf des Theorieteils genauer erläutert) greifbar machen zu können, wurde eine Skala entwickelt, welche diese misst (SIMS = Situational Motivation Scale). "The SIMS represents a brief and versatile self-report measure of situational intrinsic motivation, identified regulation, external regulation, and amotivation" (Guay, Vallerand \& Blanchard 2000: 175). Bei dieser Skala handelt es sich um eine quantitative 7-Punkt-Likert-Skala, welche von ,trifft überhaupt nicht zu' bis hin zu ,trifft voll und ganz zu' rangiert. Da es 
sich bei der Untersuchung um die aktuelle situationelle Motivation handelt, beziehen sich alle Frage-Items auf die momentane Betätigungsausführung. Die anzukreuzenden Aussagen beziehen sich entweder auf ,intrinsic motivation;, ,identified regulation', external regulation' oder ,amotivation', welches dem / der Befragten jedoch verborgen bleibt (vgl. Guay, Vallerand \& Blanchard 2000). Kritisch zu betrachten ist bei dieser Messskala die Tatsache, dass alle Aussagen bereits vorgegeben sind und die oder der Befragte keine eigenen Gedanken oder Gefühle in die Auswertung einbringen kann (siehe zum Vergleich auch Kritik an quantitativer Forschung, Kapitel 7).

Hier fügt sich eine weitere quantitative Studie in die Reihe der vorzustellenden Vergleichsstudien zum Thema Motivation ein. Brand (2006) untersucht in seinem Artikel in der Zeitschrift für Sportpsychologie die „affektive Einstellungskomponente und ihren Beitrag zur Erklärung von Sportpartizipation“ im Vergleich zur kognitiven Einstellungskomponente. Er belegt unter anderem, dass die affektive, also die gefühlsbetonte Einstellungskomponente eher für eine Sportpartizipation im Gesundheitssport verantwortlich ist als die kognitive Einstellungskomponente. Als einer der wenigen Forscher untersucht Brand hier zusätzlich auch inaktive Probanden (allerdings im Alter von 40-54 Jahren, also jünger als die Zielgruppe dieser Arbeit) mittels eines Fragebogens. Zwar findet er heraus, dass "sowohl die kognitive als auch die affektive Einstellungskomponente [...] bei sportlich aktiven Personen positiver als bei sportlich Inaktiven ausgeprägt [ist]" (Brand 2006: 152), jedoch bleiben Emotionen und Hintergründe aufgrund der Art der Befragung (schriftlicher Fragebogen) verborgen. Erschwerend kommt die Tatsache hinzu, dass Probanden lediglich eine positiv-affektive (emotionale) Einstellung dem Sporttreiben gegenüber vorweisen können, wenn sie in der Vergangenheit bereits ähnliche Vergleichserfahrungen sammeln konnten. Daher sind Nichtsportler mit negativen Schulsporterfahrungen hier deutlich im Nachteil, was die emotionale affektive Einstellungskomponente bezüglich des Sporttreibens angeht. 
Grundsätzlich erfordert ein Wiederanfang beim Sporttreiben im Seniorenalter, insbesondere von neuen und unbekannten Bewegungsausführungen, oftmals viel Geduld und Hingabe, da „ältere Erwachsene [im Vergleich zu jüngeren] geringere Fortschritte beim Erlernen neuer Fertigkeiten aufweisen" (Seidler \& Schott 2013: 19). Im Umkehrschluss fällt es älteren Erwachsenen jedoch leichter, verwandte oder ähnliche der von innen bereits bekannten und erlernten Sportarten auszuführen, da gewisse Strukturen schon im Gehirn verankert sind, auf die in solchen Fällen zurückgegriffen werden kann.

Ferner wird in einer Studie von Kolt, Driver und Giles (2004) untersucht, warum ältere Australier/innen Sport treiben. Diese Studie ähnelt dem empirischen Teil dieser Arbeit, was die Durchführung und den Inhalt betrifft. 815 durchschnittlich knapp 64-Jährige werden bezüglich der Gründe ihres Sporttreibens, bezüglich ihrer Sportarten und zu einigen demographischen Daten befragt. Der Fragebogen (Participation Motivation Questionnaire for Older Adults) beinhaltet 30 Items zu der Frage nach der Motivation und wird auf einer 3-Punkte-Skala erzeugt. Außerdem wird nach dem Alter, dem Beruf, dem Schulabschluss und den betriebenen Sportarten gefragt. Die am häufigsten genannten Sportarten sind Wandern, Golf, Schwimmen, Tennisspielen, Radfahren und Aqua-Fitness. Die wichtigsten Gründe für das Sporttreiben finden sich im sozialen und gesundheitlichen Bereich. Anerkennung und Leistungssteigerung zählen zu den unwichtigsten Gründen. Ein Vergleich mit dieser Arbeit ist inhaltlich jedoch schwierig, da der Schwerpunkt im australischen Sportsystem ein anderer ist, was beispielsweise an den genannten Sportarten zu belegen ist.

Eine weitere Studie befasst sich ebenfalls mit den Motiven zum Sporttreiben; in diesem Falle in den Vereinigten Staaten von Amerika. In ihrer Studie untersuchen Reed und Cox (2007) 981 Athleten und Athletinnen mit einem Durchschnittsalter von rund 62 Jahren. Hier muss berücksichtigt werden, dass 82\% der Befragten männlich sind. Zusätzlich zu den obligatorischen demographischen Angaben wird eine Sport-Motivations-Skala (,Sport Motivation Scale") aufgeführt, um mithilfe einer 7-Punkte-Skala eine allgemeine 
Motivation für Sport herauszufinden. (Für weiterführende Informationen zu dieser Skala inklusive einer Erweiterung bzw. Überarbeitung, siehe z.B. Mallett et al. 2007). Hinzu kommt eine weitere Skala zur spezifischen Klärung der einzelnen Gründe des Sporttreibens. Diese besteht aus 30 Items und beinhaltet ebenfalls eine 7-Punkte-Skala. Die Ergebnisse dieser Studie lassen sich, wie folgt, zusammenfassen: Frauen treiben eher Sport, um fit zu bleiben, als Männer. Außerdem lässt sich die ältere Gruppe der Befragten leichter demotivieren als die jüngere. Zudem ist den älteren Teilnehmenden die soziale Interaktion als Motiv wichtiger als den jüngeren. Ältere Frauen haben grundsätzlich mehr Spaß am Sporttreiben als ältere Männer. Ein Vergleich dieser Resultate mit den Ergebnissen aus dem noch folgenden empirischen Teil ist aufgrund der unterschiedlichen Befragungsstile sowie der anderen Zielgruppe nicht sinnvoll.

Eine andere Studie benennt weitere wichtige Faktoren, die in Bezug auf Motivation eine relevante Rolle spielen. So untersuchen Stiggelbout, HopmanRock und van Mechelen (2008) nicht nur persönliche Faktoren, wie etwa Alter, Schichtzugehörigkeit oder Sportverhalten, sondern auch kulturelle Faktoren, wie etwa den Einfluss des Trainers oder der Familie, und umweltbezogene Faktoren, wie etwa die Qualität der Sportstätten, Kosten oder Qualität der Aktivitäten an sich. Diese Studie erfasst 2.020 niederländische Seniorinnen und Senioren, welche ein Durchschnittsalter von etwa 61 Jahren aufweisen.

Außerdem werden weitere Befragungen zur Gesundheit und zum Alltagsleben erhoben, wie etwa der allgemeine Gesundheitszustand, gemessen anhand von Angaben bezüglich des Konsums von Alkohol und / oder Zigaretten. Diejenigen Sportlerinnen und Sportler, die Radfahren als Hauptsportart angeben, erzielen die höchsten Werte auf der vorgegebenen Gesundheitsskala.

Als Ergebnis werden drei verschiedene Motivationstypen von Seniorensportlerinnen und -sportlern herausgearbeitet: die Entspannen-und-GenießenGruppe („Relax and Enjoy“ (Stiggelbout, Hopman-Rock und Van Mechelen 2008: 350), die Fürsorge-und-Heil-Gruppe („Care and Cure“) und die 
Wettbewerbsgruppe („Competition“). Letztere wird lediglich von den jüngeren Teilnehmenden genannt.

In einer weiteren Studie wird die so genannte Sport Motivation Scale (Brière et al. \& Pelletier et al. 1995), ein Fragebogen zur Definition der Motivation von sporttreibenden Athleten, konzipiert. Auch hier stellt sich heraus, dass der soziale Aspekt bezogen auf die Sportmotivation eine sehr große Rolle spielt.

Obwohl es mittlerweile so viele positive Gründe für den Alterssport und einige Fördermaßnahmen gibt, wird das Angebot von einer Vielzahl an Personen immer noch nicht angenommen:

\footnotetext{
„Es besteht eine unübersehbare eklatante Diskrepanz zwischen den Idealen des auch in der Öffentlichkeit vorherrschenden sportwissenschaftlichen Alter(n)sparadigmas, seinen attraktiven Versprechen, wie Gesundheit, längeres Leben, mehr Lebensgenuss, und den erkennbaren relativ geringen Erfolgen der Aktivierung älterer und alter Menschen, insbesondere für aktiven Sport“ (Backes 2001: 296-97).
}

Oftmals sind die Gründe für die Inaktivität in der Gewohnheit zu sehen. Sind ältere Menschen es nicht gewohnt, sportlich aktiv zu sein, so bedeutet ihnen die Bewegung nicht viel, da sie die positiven Auswirkungen entweder noch gar nicht erlebt haben oder sich nicht mehr an sie erinnern können. Zudem beginnen viele ältere Menschen erst mit einer sportlichen Betätigung, wenn diese ihnen von ihrem Arzt ausdrücklich als immens wichtig nahegelegt wird und sie an erheblichen gesundheitlichen Einschränkungen leiden (vgl. Tomasch 1997).

Eine sehr aktuelle und für die zukünftige Motivationsforschung im Sport richtungsweisende Studie stammt von Sudeck, Lehnert \& Conzelmann (2011). Die Autoren befassen sich mit motivbasierten Sporttypen und stellen die so genannte ,Personorientierung' im Breitensport in den Vordergrund. Ihr konkretes Ziel ist, eine individualisierte sportliche Intervention für bestimmte Bevölkerungsgruppen zu kreieren. Ihre These deutet darauf hin, dass individuelle Gründe, warum Sport getrieben werden könnte, über den 
gesellschaftlichen Vorsätzen stehen sollten, um ein Sportprogramm so individuell ansprechend wie möglich zu gestalten. Das Ziel ihrer Studie ist die „Berücksichtigung von individuellen Beweggründen mit Blick auf die Konzeption von Sportinterventionen im Freizeit- und Gesundheitssport" von Menschen im „mittleren Erwachsenenalter“ (Sudeck, Lehnert \& Conzelmann 2011: 2).

Sie kritisieren innerhalb ihres Beitrages, dass bereits durchgeführte Studien ähnlicher Art lediglich auf Durchschnittswerten basieren und das Individuum an sich wenig Beachtung erfährt.

Methodisch gehen sie mithilfe einer so genannten Clusteranalyse (Gruppenbildung) zur Erforschung spezifischer Profile vor. Diese hat den Anspruch, zunächst stark zu individualisieren, um anschließend wieder zu verallgemeinern, damit mehrere Individuen mit ähnlichen Merkmalen gefunden werden können, welche in eine Gruppierung (Cluster) passen. Voreinschränkungen, wie z.B. körperliche Fehlfunktionen, oder auch sportliche Vorerfahrungen sowie gesundheitliche Aspekte und Sportengagement sollten stets zusätzlich abgefragt bzw. beachtet werden.

Die Zielgruppe besteht in ihrer Studie aus Angestellten einer Hochschule im Alter von 35 Jahren und älter (Durchschnittsalter = ca. 48 Jahre), wovon 19,6\% der Befragten Nichtsportler sind. Die Untersuchung ist sowohl quantitativ (Fragebogen) als auch qualitativ (Leitfadeninterview) durchgeführt worden und umfasst zusätzlich einen praktischen Sporttest zu den Themen Ausdauer, Kraft, Beweglichkeit und Koordination, aus denen schließlich mithilfe einer statistischen Auswertung die finalen Gruppierungen (Cluster) gebildet werden.

Der Fokus der Clusteranalyse liegt hier auf der "Identifikation von Zielgruppensegmenten auf Basis typischer Motiv- und Zielprofile" (Sudeck, Lehnert \& Conzelmann 2011: 6), anders als bei Gabler (2002), welcher bereits von absoluten Motivausprägungen ausgeht und nicht personorientiert erhebt.

Als Ergebnis präsentieren Sudeck, Lehnert \& Conzelmann (2011) insgesamt neun Cluster, welche die einzelnen Profile charakterisieren: 
- Cluster 1: „Kontaktfreudige Sportler/-innen“ (10)

- Cluster 2: „Figurbewusste Ästheten/-innen“ (10)

- Cluster 3: „Aktiv-Erholer/-innen“ (10)

- Cluster 4: „Erholungssuchende Fitnessorientierte“ (10)

- Cluster 5: „Sportbegeisterte“ (10)

- Cluster 6: „Gesundheits- und Figurorientierte“ (10-11)

- Cluster 7: „Figurbewusste Gesellige“ (11)

- Cluster 8: „Figurorientierte Stressregulierer/-innen“ (11)

- Cluster 9: „Erholungssuchende Sportler/-innen“ (11)

Diese geben nun Auskunft über die individuellen Wünsche und Bedürfnisse der Befragten und mithilfe dieser Auflistung ist ein individuell zugeschnittenes Trainingsprogramm leichter möglich als ohne jegliche Informationen bezüglich der Zielgruppe.

Während in der früheren Literatur oftmals lediglich gesellschaftlich relevante Motivationsgründe, wie z.B. Gewichtsreduktion, Gesundheit oder Geselligkeit, im Zentrum der Motivationsforschung standen (siehe Kapitel 4.3 bei Singer (1981) oder Fuchs (1997)), wird dank dieser neuen Studie deutlich, dass Motive (hier als Profile bezeichnet) oftmals nicht eingleisig zu definieren sind, sondern in den meisten Fällen sich mit weiteren Motivblöcken überlappen, sodass die oben genannten Mischformen entstehen. Demnach ist von hoher Bedeutung, dass der Fokus auf das Individuum gelegt wird, um die einzelnen Bedürfnisse beim Thema Sporttreiben herausfiltern zu können. Daher „lassen sich substanziell höhere Steigerungen des Wohlbefindens erkennen, wenn Personen an einem auf ihren Sport-Typ zugeschnittenen Sportprogramm teilnehmen, welches seine Inhalte und Maßnahmen besonders auf die individuellen Motive und Ziele abstimmt" (Sudeck, Lehnert \& Conzelmann 2011: 16). (Inwieweit dies in der Praxis in größeren Gruppen möglich wäre, ist bisher als unerforscht einzustufen.)

Des Weiteren wird ebenfalls deutlich, dass frühe positive Sporterfahrungen in den meisten Fällen zum sportlichen Grundbedürfnis im Alltag beitragen, sodass anzunehmen ist, dass Menschen mit schönen Schulsporterinnerungen grundsätzlich eher motiviert sind, auch noch im höheren Alter Sport zu treiben. 
Aus den hier vorgestellten aktuellen Studien ergibt sich die Begründung für die methodische Vorgehensweise in dieser Dissertation: Zum einen bedarf es bei der Wahl der Forschungsmethode eines Schwerpunktes auf qualitativer Sozialforschung, da die veröffentlichten Studien oftmals ausschließlich auf quantitativen Daten basieren und nur in Einzelfällen qualitative Forschungsmethoden ergänzend durchgeführt werden. Zum anderen ist bei der Wahl der Stichprobe der Vergleich von Wiedereinsteigern und NichtSportlerinnen und -Sportlern in einer Altersklasse von 60 Jahren und mehr ein Novum in der Literatur. Kombiniert mit dem Fokus auf der Selbstpräsentation der Probandinnen und Probanden sowie ihrem individuellen Verständnis vom Sporttreiben ergibt sich die Kernthematik dieser Arbeit, welche im empirischen Teil vertieft wird (siehe Seite $80 \mathrm{ff}$.).

Um die hier vorgestellten aktuellen Studien zu verstehen und auch die weiteren Inhalte dieser Arbeit nachvollziehen und einordnen zu können, bedarf es der Erläuterungen bestimmter Begrifflichkeiten, welche im folgenden Kapitel dargelegt werden.

\section{Seniorensport / Alterssport}

\subsection{Begriffsbestimmung ,Seniorenalter'}

Die so genannte ,Gerontologie', ein 1929 eingeführter Begriff, befasst sich mit der Lehre des Alterns beziehungsweise mit der Alternsforschung (vgl. Singer 1981). Während der Begriff des Alterns einen prozessartigen Vorgang bzw. eine Entwicklung beschreibt, ist das Alter selbst „dessen Ergebnis [...], jener Zustand, der durch das Altern erreicht wird“" (Meusel et al. 1980: 15). Während des Alterns werden Menschen weniger adaptationsfähig auf Reize, sowohl auf interne als auch auf externe (vgl. Denk, Pache \& Schaller 2003). Die Voraussetzungen für ein langes Leben hängen von unterschiedlichen Faktoren ab. So spielen beispielsweise genetische, biologische, soziale und ökologische 
Faktoren eine Rolle, wie auch Bildung, Ernährung, körperliche Betätigung und Hygiene (vgl. Werle, Woll \& Tittlbach 2006).

Der exakte Eintritt in das Seniorenalter ist in der Literatur unterschiedlich definiert. In einigen Werken wird der Beginn des Seniorenalters bereits mit 50 Jahren, in anderen Werken mit 60 Jahren oder mit Eintritt in den Ruhestand angegeben (vgl. Dahlhaus 2007). Hier wird bereits deutlich, dass eine dynamisierte Herangehensweise an das Alter in der heutigen Zeit sinnvoll erscheint, was im Kapitel 5 zum demographischen Wandel erneut aufgegriffen wird.

Bezüglich des Seniorenalters im Sportbereich gibt es wieder unterschiedliche Werte. So beschrieb Lorentz (1937) damals die Altersspanne von 35-55 Jahren als Seniorenalter im Sport und die Gruppe mit der Altersspanne von 55-75 Jahren bereits als Altsenioren und Altseniorinnen (vgl. Singer 1981).

Grundsätzlich ist es wichtig, einen Unterschied zwischen den verschiedenen Definitionen des Seniorenalters zu machen, je nachdem, ob es sich um den Leistungssport oder um den Freizeit- und Gesundheitssport handelt. Zur Vereinfachung wird im Folgenden das kalendarische Alter der Seniorinnen und Senioren, die am Alterssport teilnehmen, auf jene, „die das 60. Lebensjahr erreicht haben bzw. in den Ruhestand eingetreten sind“, (Tomasch 1997:37) festgelegt.

Zusätzlich kann der Begriff des Alters in weitere Subkategorien unterteilt werden. Somit bezieht sich das biologische Alter hauptsächlich auf den „Zustand des Organismus“ (Tomasch 1997: 7), während das psychologische Alter eine subjektive Einschätzung des aktuell gefühlten Alters beschreibt und das soziologische Alter eher eine Widerspiegelung der Sicht der Gesellschaft auf das Individuum darstellt (vgl. Tomasch 1997). Das kalendarische Alter ist jedoch nicht alleinig ausreichend, um beispielsweise die Leistungsfähigkeit eines Trainierten oder Nichttrainierten einschätzen zu können. Hinzu kommt die Tatsache, dass viele Menschen ab einem gewissen Alter (durchschnittlich ab 25 Jahren) die Tendenz aufweisen, ihr subjektives Alter deutlich geringer einzuschätzen als ihr eigentliches kalendarisches Alter (vgl. Staudinger 2012). 
Um Aussagen über einen Fitnesszustand eines Menschen zu machen, reicht daher die Angabe über das kalendarische Alter in keiner Weise aus - im Gegenteil: Trainierte Seniorinnen und Senioren weisen weit höhere Fitnesswerte beispielsweise im Ausdauerbereich auf als untrainierte Seniorinnen und Senioren und können in vielen Fällen mit untrainierten Jüngeren verglichen werden (vgl. Schlicht \& Schott 2013).

Nichtsdestotrotz ist das Altern neben den biologischen und gesellschaftlichen Aspekten „im Wesentlichen individuelle Gestaltung und Selbstbestimmung“ (Perrig-Chiello 2008: 41). Dies bedeutet, dass Altern zwar ein irreversibler biologischer Prozess ist, welcher jedoch durch den eigenen Umgang auf- oder abgewertet werden kann. Dass diese Prozedur nicht ohne Überwindungen und Barrieren durchlebt und geschafft werden kann, ist ohne Zweifel als selbstverständlich einzustufen, dennoch hilft eine positive Lebenseinstellung im Umgang mit dem Älterwerden.

Da das Altern unumgänglich ist und mit Charakteristika wie beginnenden Falten, grauen Haaren, veränderten Bewegungsmustern, einem Abfall der körperlichen Leistungsfähigkeit und einer Reduzierung einiger Sinne beschrieben wird, ruft dieser Funktionsverlust oftmals eine negative Konnotation hervor: „Das eigene Altern wird zunächst durch den alternden Leib wahrgenommen, der gängigen Schönheitsnormen nicht mehr entspricht, abverlangte Leistungen versagt und zum Teil Schmerzen bereitet" (Kolb 1999: 263). Die Gesellschaft erschafft zum einen ein Bild vom unglücklichen Altern (,Anti-Aging-Bilder') und existierende Altersstereotypen erschweren einen unbeschwerten Umgang mit dem Älterwerden (vgl. Wollny 2002 \& PerrigChiello 2008). Zum anderen präsentieren die Medien immer häufiger Werbung mit jung gebliebenen Alten, welche meist faltenlos in die Kamera lächeln und ebenfalls ein übertriebenes und nicht realistisches Bild projizieren (vgl. Schlicht \& Schott 2013).

Daher erscheint es umso wichtiger, im Alter einen Weg zu finden, um diesen Klischees entgegenzuwirken. Noch vor drei Jahrzehnten waren einige ältere Menschen der Meinung, „Sport stärke die Gesundheit; man bleibe fit, jung, 
schlank; komme an frische Luft; bleibe elastisch“ (Singer 1981: 86). Ob und inwiefern dieser Gedanke noch heute aktuell ist, wird sich im Verlauf dieser Arbeit zeigen. Jedoch bleibt wichtig zu erwähnen, dass eine Veränderung im Verhalten nicht auftritt, „weil jemand älter wird, sondern weil Ereignisse eintreten, die dann Verhaltensveränderungen bewirken“ (Wollny 2002: 78). Somit bleibt die Bewältigung und das Umgehen mit dem Älterwerden letztendlich die Aufgabe eines jeden (gesunden) Individuums.

Des Weiteren können die Älteren anhand ihres aktiven Leistungsniveaus genauer kategorisiert werden. So unterteilt Spirduso (1995: 375) die Älteren in folgende Kategorien: „Die körperlich Abhängigen“, „die Gebrechlichen“, „die Unabhängigen“, „die Fitten“ und „die körperliche Elite“. Im nächsten Unterkapitel geht es um die Begriffsbestimmungen rund um den Senioren- bzw. Alterssport. Die teilnehmenden Seniorinnen und Senioren sind laut Spirduso die Fitten, denn sie trainieren mehrmals in der Woche „für ihre Gesundheit, für ihr Vergnügen und Wohlbefinden“ (Spirduso 1995: 375).

\subsection{Begriffsbestimmung, Seniorensport'}

Der Begriff des Seniorensports ist ein in der Literatur oftmals unterschiedlich verwendeter Ausdruck, welcher verschiedene Interpretationsmöglichkeiten zulässt. Im Wettkampfbereich beginnt der Seniorensport bereits ab einem Alter von 30 Jahren (vgl. www.leichtathletik.de). Insbesondere im Leichtathletikbereich werden sowohl nationale als auch internationale Meisterschaften im Seniorensport durchgeführt. Der so genannte SeniorenLeichtathletik-Weltverband (World Masters Athletics $=$ WMA) wurde im Jahr 1977 gegründet und ist zuständig für die „Organisation, Leitung und Verwaltung der Leichtathletik für Frauen ab deren 35. und für Männer ab deren 35. Lebensjahr“(http://www.world-mastersathletics.org/files/laws_rules/satzung.pdf).

Jüngste Ergebnisse verdeutlichen, dass Seniorinnen und Senioren bis ins hohe Alter an den Weltmeisterschaften teilnehmen: Im Kurzstreckenlauf über 60 Meter der Damen nahm eine 95-jährige Kanadierin teil und bei den Herren 
nahm bei gleicher Disziplin ein 97-jähriger Italiener teil (vgl. http://www.worldmasters-athletics.org/files/results/2014budapest/results.pdf).

Auch im nationalen Deutschen Leichtathletikverband (DLV) existiert eine Senioren-Bestenliste, welche von Jörg Reckemeier zusammengestellt wurde. Hier lassen sich ebenfalls Rekordhalterinnen von über 80 Jahren und Rekordhalter von über 95 Jahren in sämtlichen Disziplinen finden (vgl. http://www.leichtathletik.de/image.php?AID $=43286 \& V I D=0)$. Darüber hinaus ist das Durchführen des Sportabzeichens bis ins hohe Alter eine Herausforderung, welche von vielen Seniorensportlern jährlich genutzt wird (vgl. http://www.dosb.de/de/sportentwicklung/sportabzeichen/). So erwarben im Jahr 2013 insgesamt 52.071 Erwachsene im Alter von 60 und 60+ das Sportabzeichen in Deutschland (vgl. http://www.deutsches-sportabzeichen.de).

Der Begriff des Seniorensports wird in dieser Arbeit von nun an synonym mit dem Begriff des Alterssports verwandt. Da es beim Alterssport vornehmlich um die Erhaltung der Gesundheit geht, anstatt um die Erbringung einer bestimmten Leistung, wird auch häufig der Begriff des Gesundheitssports gewählt. Die Gesundheit an sich wird als „prozesshaftes Geschehen aufgefasst, das sich im aktuellen Bezug herausbildet" (Werle, Woll \& Tittlbach 2006: 27). Die Definition von Gesundheit nach der WHO, in der Gesundheit als ein „Zustand des vollkommenen körperlichen, geistigen und sozialen Wohlbefindens [...]" (Tomasch 1997: 8) charakterisiert wird, gilt heutzutage als veraltet beziehungsweise ungenau und zu einseitig. Stattdessen kristallisiert sich das Gesundheitsmodell von Antonovsky (1997), welches die starre kurative Medizin als alleiniges Heilmittel in Frage stellt, als angemessen und sinnvoll heraus, auf das im nächsten Unterpunkt genauer eingegangen wird (vgl. Fuchs 2003).

Grundsätzlich gilt, dass "Gesundheit kein Besitz, sondern ein sich stets verändernder Zustand ist, der nur aktiv zu erhalten oder wieder herzustellen ist" (Dahlhaus 1997: 41). Der Gesundheitsbegriff umfasst also nicht nur ein persönliches Wohlbefinden, sondern auch eine Selbständigkeit im Alltag, Offenheit für neue Erfahrungen, die Fähigkeit zum Umgang mit Konflikten sowie 
die Fähigkeit zur Kompensation von Verlusten oder körperlichen Einschränkungen (vgl. u.a. Daugs et al. 2001; Fuchs 2003).

Der Alterssport als solcher existiert zwar beispielsweise in Sportvereinen, Volkshochschulen, Altenheimen oder Kirchengemeinden, jedoch ist seine Bedeutung für die Menschheit erst innerhalb der letzten Jahrzehnte als immens wichtig erkannt worden (vgl. Singer 1981). Diese deutliche Entwicklung spiegelt sich in der aktuellen Beliebtheit und Notwendigkeit des Seniorensports wider. Dies ist der Grund, warum es mittlerweile auch nicht mehr als „exotisch [gilt], in fortgeschrittenem Alter Sport zu treiben; ein Alterssportler wird kaum noch belächelt, so wie das noch vor etwa 20 Jahren häufig der Fall war“ (Dahlhaus 2007: 10). Dennoch gilt Alterssport immer noch nicht als Selbstverständlichkeit und die propagierte sportliche Lebensweise im Alter gehört nicht in jedem Seniorenkreis zum Programm (vgl. Kolb 1999).

Eine eindeutige Definition des Begriffes des Sports ist nahezu unmöglich: „Seit Beginn des 20. Jahrhunderts hat sich Sport zu einem umgangssprachlichen, weltweit gebrauchten Begriff entwickelt. Eine präzise oder gar eindeutige begriffliche Abgrenzung läßt sich deshalb nicht vornehmen" (Röthig \& Prohl 2003:149). Dennoch soll versucht werden, im Folgenden den Sportbegriff, bezogen auf Seniorinnen und Senioren, klarer einzugrenzen und zumindest einen Versuch der Definition, wie er für diese Arbeit von Belang sein wird, vorzunehmen.

Heutzutage wird der Fokus innerhalb des Seniorensports auf die körperlichen Veränderungen im Alter gelegt, bei denen besonders die „im Sport angelegten Möglichkeiten zur Bekämpfung der mit dem Alter einhergehenden Defizite genutzt und Abbauprozesse ausgeglichen werden sollen“ (Kolb 1999: 157).

Das wohl größte Ziel des Seniorensports ist die lange körperliche Unabhängigkeit im Gegensatz zur Hilfsbedürftigkeit. Dazu gehört eine gute Fitness, also die „Fähigkeit, die Anforderungen des Alltags ohne stärkere Ermüdung zu 
erfüllen, die Freizeitinteressen wahrzunehmen und auf unvorhergesehene Situationen erfolgreich zu reagieren" (Israel 1995: 221 zit. nach Kolb 1999: 165). Neben den körperlichen Zielen des Alterssports existieren psychische Ziele, wie beispielsweise ein verstärktes Selbstvertrauen, und soziale Ziele, wie beispielsweise die Zugehörigkeit zu einer Gruppe (vgl. Kolb 1999).

Im Vordergrund des Alterssports steht immer der präventive Gedanke, also die „Krankheitsvermeidung beziehungsweise -verhütung“ (Werle, Woll \& Tittlbach 2006: 30). Hierbei wird die sportliche Betätigung als eines der wirksamsten Mittel bezeichnet, um genau diesen präventiven Charakter zu erfüllen und das Risiko vor Krankheiten oder Unfällen zu reduzieren (vgl. Meusel et al. 1980). Daher wird der Sportbegriff in dieser Arbeit niemals im klassischen Sinne als Wettkampf interpretiert, sondern der Fokus wird auf die aktive körperliche Betätigung bzw. sportliche Aktivität gelegt, das heißt auf die "geplanten, bewusst aus dem Alltag herausgenommenen körperlichen Aktivitäten in der Freizeit, welche die Handlungsmuster des Sports übernehmen [...]" (Schlicht \& Schott 2013: 16). Hier ist explizit hervorzuheben, dass ein aktiver Lebensstil, wie das Zurücklegen bestimmter Strecken mit dem Fahrrad oder zu Fuß zum Zweck einer anderen Tätigkeit, wie beispielsweise einkaufen, in dieser Arbeit nicht unter den Sportbegriff fällt.

Als präventives Mittel kann das Sporttreiben laut einer Studie von DeFina et al. (2013) auch vor demenziellen Erkrankungen schützen. Die beteiligten Forscherinnen und Forscher untersuchten in ihrer Studie den Zusammenhang von Fitness-Level und späteren Demenzerkrankungen mit 19.458 Erwachsenen über durchschnittlich 25 Jahre. Schlussfolgernd stellen sie fest, dass die Wahrscheinlichkeit, im höheren Alter an Demenz zu erkranken bei Menschen mit geringerem Fitness-Level höher ist als bei Menschen mit höherem FitnessLevel. Sporttreiben als Primärprävention von Alzheimer und anderen Demenzerkrankungen erscheint hier als durchaus sinnvoll und lohnenswert. Unklar bleibt jedoch, wie viel wöchentlich trainiert werden muss und wie ein 
Training genau aussehen soll, damit ein ausreichend hohes Fitness-Level erreicht wird, um schließlich wirksam präventiv Erfolge zu erzielen.

Generell muss festgehalten werden, dass der Seniorensport den Alterungsprozess selbstverständlich nicht komplett aufhalten, inn jedoch nach hinten verschieben kann und im Normalfall für ein besseres persönliches Wohlbefinden sorgt (vgl. Dahlhaus 1997). Dieses führt mit der Aufrechterhaltung des physischen und psychischen Wohlbefindens zu einem erfolgreichen Altern (vgl. Schlicht \& Schott 2013).

Da allerdings durchschnittlich „bei Erwachsenen die Beliebtheit des Sports als Freizeitbeschäftigung mit zunehmendem Alter ebenso abnimmt wie die Häufigkeit des Sporttreibens, der Sportvereinsmitgliedschaft und des Besuches von Sportveranstaltungen“ (Singer 1981: 76), sind die Bedürfnisse und Wünsche der Älteren zu berücksichtigen, wenn es um die Planung und Durchführung des Alterssports geht. So existieren neben den sozialen Aspekten weitere Bedürfnisse, wie zum Beispiel ausreichend Erholung, genügend Informationen und ein strukturierter Ablauf und dementsprechend eine möglichst eigenständige Gestaltung der Zeit (vgl. Dahlhaus 1997).

Sind diese Aspekte nicht gegeben, ist die Wahrscheinlichkeit groß, dass die Seniorinnen und Senioren sich gegen das Sporttreiben entscheiden und sich dadurch in einen so genannten Teufelskreis begeben (vgl. auch Schlicht \& Schott 2013):

"As people age, they become less active. The less active they are, the less physical ability and endurance they have. The less physical ability they have, the less inclined they are to be physically active. And the less active they are, the more physical capacity they lose. The less they move, the less they can move. [...] The result of this vicious cycle is a steady deterioration of function" (Spirduso 1995: 355f.).

Ein gesundheitsbewusstes Verhalten ist daher unumgänglich für ein erfolgreiches Altern (vgl. Kolb 1999). Kruse (2001: 555f.) beschreibt die vier 
wichtigsten Punkte in Bezug auf das Gesundheitsverhalten: „eine aktive Lebensführung“, „das gesundheitliche Wohlbefinden“, sowohl objektiv als auch subjektiv, „die Vermeidung von Risikofaktoren“, wie etwa Rauchen, Alkohol oder Übergewicht, sowie „eine positive Lebenseinstellung“ inklusive freudiger Erwartung im Hinblick auf die Zukunft.

Indirekt finden sich diese Aspekte in Kombination mit dem Nennen von sportlicher Betätigung in einem Zitat aus Müller, Rösch \& Wischmann (1979: 107), welches trotz des frühen Erscheinungsdatums heute nicht an Aktualität verloren hat:

„Durch Sport kann man [...] nicht nur dem Leben Jahre, sondern den Jahren Leben hinzufügen.“

Wie wörtlich diese Aussage zu nehmen ist, wird in den folgenden Unterpunkten näher untersucht, nachdem das Gesundheitsmodell von Antonovsky (1997) genauer beschrieben worden ist, welches notwendig ist, um das Thema Gesundheit besser zu verstehen und zu betrachten.

\subsection{Der Begriff der Salutogenese nach Antonovsky (1997)}

Grundsätzlich gibt es, was das Verständnis von Gesundheit bzw. Krankheit angeht, zwei gegensätzliche Sichtweisen: zum einen das pathogenetische Grundverständnis, nämlich die Behandlung von Kranken, um diese vor Chronifizierung oder Tod zu schützen, und zum anderen die Salutogenese, welche versucht, die Menschen so gesund wie möglich zu halten und sie vor möglichen Krankheiten zu bewahren.

Die Salutogenese plädiert erstens für eine Abschaffung der strikten Unterteilung von ,gesund' und ,krank' und sieht den Menschen vielmehr auf einem „Gesundheits-Krankheits-Kontinuum“ (Antonovsky 1997: 27). Dies bedeutet, 
dass sich jedes Individuum auf einem unterschiedlichen Punkt auf diesem Kontinuum befindet, welcher jederzeit untersuchbar ist. Hier ist also wichtig, dass der Mensch nicht ,gesund' oder ,krank' ist, sondern sich eher in Richtung Gesundheit oder eher in Richtung Krankheit auf einer Ebene befindet und niemals komplett ,krank' oder komplett ,gesund' ist.

Überdies versucht sich der salutogenetische Ansatz von einer starren Untersuchung der Ursachen von Krankheiten abzuwenden. Hier soll also nicht sofort und in jedem Fall überprüft werden, welches Krankheitsbild vorliegt, sondern vielmehr nach folgendem Grundsatz gehandelt werden: „Welche Faktoren sind daran beteiligt, daß man seine Position auf dem Kontinuum zumindest beibehalten oder aber auf den gesunden Pol hin bewegen kann?" (Antonovsky 1997: 29)

Um dieses Ziel zu erreichen, werden die so genannten ,Coping-Ressourcen' in den Mittelpunkt gerückt, also die Mittel, durch die mit Problemen erfolgreich umgegangen werden kann.

Ein weiterer und letzter wichtiger Faktor im Zusammenhang mit Salutogenese ist die Bewertung von Stressoren, also Auslösern, die für Stress verantwortlich gemacht werden. Diese sind alltäglich, jedoch nicht unbedingt nachteilig und werden von jedem Individuum als unterschiedlich erfasst.

Um die salutogenetische Vorstellung für den Verlauf des Lebens deutlicher veranschaulichen zu können, stellt Antonovsky (1997) eine aussagekräftige Metapher vor, nämlich die so genannte „Flussabwärts-Perspektive“ (91).

Diese erläutert er folgendermaßen: Der Fluss dieser Perspektive ist als Strom des Lebens zu interpretieren. Nun stellen sich einige Fragen. Was passiert flussabwärts oder wer stößt hinzu? Wahrscheinlich gibt es am Ufer keine Sicherheit. Oftmals ist der Fluss verschmutzt und nicht immer sauber und gereinigt. Was passiert an Gabelungen oder Abzweigungen und bei leichter Strömung oder Stromschnellen? 
Unter Berücksichtigung all dieser Überlegungen und Unsicherheiten stellt sich nun die in Bezug auf die Salutogenese alles entscheidende Frage:

„Wie wird man, wo immer man sich in dem Fluß befindet, dessen Natur von historischen, soziokulturellen und physikalischen Umweltbedingungen bestimmt wird, ein guter Schwimmer?" (Antonovsky 1997: 92).

Dies bedeutet also, dass der Mensch trotz diverser Unannehmlichkeiten und Rückschläge stets seinen positiven Weg durch das Leben finden und meistern kann, abhängig vom sozialen Umfeld, der individuellen Willenskraft und dem Selbstbewusstsein. Um genau diese Aspekte geht es im nächsten Kapitel, nämlich um das Konzept des Kohärenzgefühls, da die Leistung des ,Schwimmers' genau von diesem Konzept abhängt.

\subsubsection{Das Konzept des Kohärenzgefühls}

Das Kohärenzgefühl, im englischen Original als "sense of coherence" (Antonovsky 1997: 36) bezeichnet und daher auch mit SOC abgekürzt, hängt davon ab, in welchem Ausmaß ein Mensch das Leben emotional als sinnvoll empfindet.

Ein starkes SOC liegt dann vor, wenn Probleme und Unannehmlichkeiten als Herausforderung gesehen und akzeptiert werden. Diese sind stets mit Emotionen verbunden. Ein schwaches SOC liegt vor, wenn ein Mensch glaubt, wenig Wichtiges und Schönes im Leben zu haben, sondern eher Unwichtiges und Mühsal, auf die er gern verzichten würde.

Das Kohärenzgefühl wird zudem mit Hilfe von drei weiteren Charakteristika näher definiert.

Das SOC ist die Fähigkeit, Zusammenhänge des Lebens zu verstehen. Es ist die Überzeugung, dass man das eigene Leben gestalten kann und es ist der Glaube, dass das Leben einen Sinn hat (vgl. Antonovsky 1997). 
Diese drei Aspekte überschreibt Antonovsky (1997) mit jeweils einem Wort. Erstens nennt er die Verstehbarkeit, also das Auftreten von Stimuli im Verlauf des Lebens, welche strukturiert, vorhersehbar und erklärbar sind. Diese Stimuli stammen aus der inneren und äußeren Umgebung und umfassen Stationen des Lebens, wie zum Beispiel den Besuch der Schule und eventuell der Universität. Hier werden also Zusammenhänge des Lebens vorausgesagt und verstanden.

Zweitens erläutert Antonovsky die Handhabbarkeit, also ein ausreichendes Ausmaß an zur Verfügung stehenden Ressourcen, um den Anforderungen, die diese Stimuli stellen, zu begegnen. Hier ist als Beispiel der Umgang mit Stresssituationen zu nennen, und zwar in unterschiedlichem Maße und mit verschiedenen Methoden, je nach vorhandenen Ressourcen.

Als dritten Begriff beschreibt der Autor die Bedeutsamkeit, also die Bedeutung von Anforderungen, welche Herausforderungen sind, für die sich Anstrengungen und Engagement lohnen. Hier wird auf die Bedeutung in gewissen Situationen abgezielt und im Großen und Ganzen auf den Glauben an den Sinn des Lebens.

Die Grundziele des SOC sind zum einen der Umgang mit Stress, ohne sich dabei negativ beeinflussen zu lassen. Zum anderen liegt der Schwerpunkt erneut auf der Gesundheitsförderung im Gegensatz zum Hervorrufen von Krankheiten. Ein weiteres Ziel sind die unterschiedlichen Reaktionen, die abgerufen werden können, um eine Situation angemessen bewältigen zu können. Das Kohärenzgefühl kann also grob mit Lebenseinstellung gleichgesetzt werden, wobei es sicherlich sehr viel komplexer ist.

Je weiter sich eine Person auf dem positiven Teil des Gesundheits-KrankheitsKontinuums befindet, desto wahrscheinlicher werden Lebenserfahrungen gemacht, die das SOC stark machen und umgekehrt. 


\subsubsection{Typen von Stressoren}

Es gibt laut Antonovsky drei Hauptgruppen von Stressoren, die für das Auftreten von Stresssituationen verantwortlich sind.

Einerseits sind die so genannten chronischen Stressoren zu nennen, welche langlebig sind, zum Beispiel Depressionen und andere psychische Krankheiten.

Andererseits gibt es Stressoren, die aus wichtigen Lebensereignissen resultieren, also zum Beispiel ein Todesfall in der Familie, eine Scheidung oder eine Kündigung.

Als letzten Typ von Stressoren bezeichnet Antonovsky akute tägliche Widrigkeiten, wie zum Beispiel Unfälle, Beleidigungen oder das Durchfallen bei Prüfungen. Die Relevanz für das SOC nimmt $a b$, d.h. die chronischen Stressoren haben die größte Einwirkung auf das SOC im Vergleich zu den akuten täglichen Widrigkeiten. Dazwischen befinden sich die wichtigen Lebensereignisse.

\subsubsection{Stressoren als generalisierte Widerstandsressourcen}

Um mit Stress umgehen zu können, gibt es verschiedene Möglichkeiten, die individuell stets unterschiedlich sind. Die Hauptauswirkung von Stressoren ist die Erzeugung eines Spannungszustandes, welcher erst einmal als neutral betrachtet werden soll.

Hinzu kommt nun ein weiteres Element der Salutogenese, nämlich die generalisierten Widerstandsressourcen, auch mit GRR (Generalized Resistance Ressources) abgekürzt. Hierbei gibt es zwei Faktoren, die individuellen und die sozialen Faktoren. Die individuellen Faktoren sind beispielsweise Intelligenz oder Bewältigungsstrategien. Die sozialen Faktoren setzen sich aus dem sozialen Umfeld und den finanziellen Möglichkeiten zusammen. All diese Ressourcen stehen jedem Menschen zur Verfügung und sollen idealerweise die Widerstandsfähigkeit einer Person erhöhen. 
Es gibt zwei Hauptaufgaben der generalisierten Widerstandsressourcen: erstens das Schaffen eines starken Kohärenzgefühls und zweitens das Herstellen eines direkten Umgangs mit Stressoren, also das so genannte ,Coping'.

Das negative Gegenteil der GRRs sind die generalisierten Widerstandsdefizite, abgekürzt mit GRD (Generalized Resistance Deficits). Diese schwächen das SOC und verhindern das erfolgreiche Umgehen mit Stresssituationen. Stress wirkt sich hier also negativ aus.

Ein Stressor ist überdies ein Merkmal, welches Entropie in ein System bringt. Hiermit ist eine Lebenserfahrung gemeint, welche durch Inkonsistenz, Unteroder Überforderung und fehlende Teilhabe an Entscheidungsprozessen gekennzeichnet ist. Die Entropie beschreibt demzufolge eine Art Unsicherheit eines Einzelnen.

Ähnlich wie bei dem Gesundheits-Krankheits-Kontinuum ergibt sich auch hier eine entsprechende Darstellung. Ein Stressor bringt Entropie in das System und sorgt für einen Spannungszustand. Dieser Stressor wirkt ein auf das Kontinuum, also auf die Achse von GRDs hin zu GRRs. Befindet sich die Person nah am positiven Ende des Kontinuums, also stärker in Richtung der generalisierten Widerstandsressourcen, wird der Stressor als nicht schädlich aufgefasst und $z u$ einem positiven Stressor umgewandelt. Dies prägt erneut das positive SOC und bietet keine weiteren Probleme mit dem zuvor erzeugten Spannungszustand. 


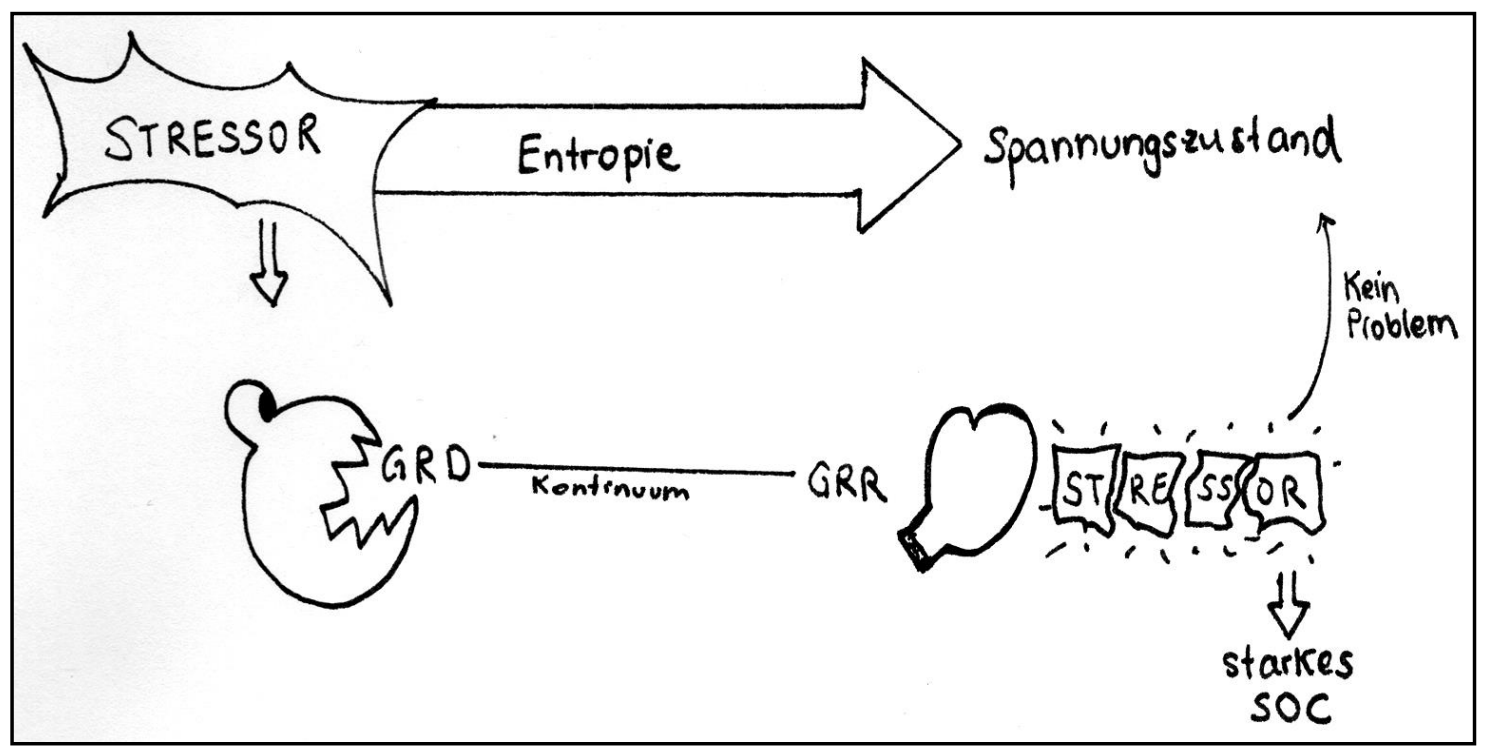

Abbildung 1: Schaubild zur Erläuterung der GRRs und GRDs (Rita Spiller)

\subsubsection{Kontrollstudien zu Antonovskys Theorie der Salutogenese}

Das Konzept der Salutogenese und die von Antonovsky entwickelten Fragebögen zur Erfassung der Lebensorientierung durch das Kohärenzgefühl (SOC) eignen sich als interkulturelles Instrument zur Erforschung des SOC. Die beiden Varianten des Fragebogens (mit 29 Items und 13 Items) wurden in viele Sprachen übersetzt und in unterschiedlichen Ländern angewandt (vgl. Lindström \& Eriksson 2005). Darüber hinaus existieren einige weitere, leicht abgewandelte Versionen, die auf der Grundlage von Antonovskys Fragebögen basieren und für bestimmte Studienzwecke modifiziert wurden (vgl. Lindström \& Eriksson 2005).

Lindström und Eriksson (2005) untersuchen eine Vielzahl an Studien, welche mit den Original-Fragebögen oder modifizierten Fragebögen zur Erfassung des SOC arbeiten, um zu testen, ob Antonovskys Hypothesen auch in der empirischen Anwendung verifiziert werden können. Unter anderem beschreibt Antonovsky das SOC als weitestgehend stabil über die Lebensjahre, wenn es 
anfänglich einen hohen Wert verzeichnet. Werden die Werte des SOC jedoch innerhalb verschiedener Altersgruppen verglichen, fällt auf, dass ältere Menschen tendenziell einen erhöhten Wert des SOC aufweisen im Vergleich zu jüngeren Forschungsteilnehmerinnen oder -teilnehmern. Die Autoren vermuten, dass dies aufgrund der positiven Entwicklung und Reifung des SOC über viele Jahre des Wohlbefindens entsteht. Im Zuge dessen erlernen Menschen, mit stressigen Situationen umzugehen und sich dementsprechend wohlzufühlen.

Das persönliche Wohlbefinden ist eng verknüpft mit der Lebensqualität, welche wiederum als relevant für das Gesundheitswesen (Public Health) einzustufen ist. In einem anderen Aufsatz vermuten Eriksson und Lindström (2007), dass das SOC eine Auswirkung auf die Lebensqualität hat. Die Lebensqualität wird als ein Konzept verstanden, welches durch einige Merkmale charakterisiert wird: „personal well-being, relations with other people, social, communal, civic activities, personal development and fulfilment, positive mental health [...]" (Eriksson \& Lindström 2007: 939). Hier wird deutlich, dass die Lebensqualität zwar viel durch das eigene Empfinden geprägt wird, jedoch auch durch den Einfluss von anderen im sozialen Kontext beeinflusst wird. Zur Überprüfung der eingangs gestellten Hypothese werden Studien mit gesundheitlich eingeschränkten Personen (z.B. durch Herzkrankheiten) verglichen. Grundsätzlich lässt sich festhalten, dass das SOC die Lebensqualität beeinflusst: Je stärker das SOC, desto höher die Lebensqualität (für junge wie ältere Probanden). Als kritisch zu bemerken ist allerdings, dass nicht in allen Studien die Lebensqualität klar definiert wurde und so die Ergebnisse in diesen Fällen mit Vorsicht und evtl. limitiert betrachtet werden sollten (vgl. Eriksson \& Lindström 2007). Nichtsdestotrotz gilt als sicher, dass das SOC eine Wirkungsvariable für die Lebensqualität verkörpert, sodass ein starkes $\mathrm{SOC}$ eng verknüpft ist mit einer guten Lebensqualität.

Überdies behauptet Antonovsky in einer anderen Hypothese, dass ein starkes SOC Einfluss auf die physiologische Gesundheit von Menschen hat. Zur Überprüfung dieser Hypothese entwickelten Flensborg-Madsen, Ventegodt und 
Merrick (2006) in einer Pilotstudie mit 100 Teilnehmerinnen und Teilnehmern eine neue SOC-Skala mit lediglich 9 Items. Während die meisten Studien einen Fokus auf die Psyche in Kombination mit dem SOC legen, wird hier explizit auf die Physis geblickt. Nach Auswertung der Pilotstudie kommt es zu dem von Antonovsky abweichenden Ergebnis, da die Hypothese falsifiziert wurde. Es herrscht demnach eine niedrige Korrelation zwischen physischer Gesundheit und dem SOC. Als Erklärung schlagen die Autoren vor, dass in ihrem Fragebogen lediglich Fragen zu Kognitionen und das Bewusstsein betreffende Einstellungen gestellt wurden, nicht aber Fragen, welche emotionale Aspekte beinhalten. In Antonovskys Originalfragebogen sind jedoch Fragen dieser Art enthalten, welche auf die Gefühle und Emotionen der Befragten abzielen. Hieraus entnehmen die Autoren, dass emotional basierte Fragetypen einen größeren Bezug zur physischen Gesundheit darstellen können. Aus diesem Grund (nämlich aufgrund der Abstinenz solcher Fragetypen in der eigenen Studie) ist diese Korrelation nicht deutlich zu erkennen: „Our hypothesis also explains why the various studies investigating associations between the 29-item SOC scale and physical health have found modest associations at most, since the 29-item SOC scale mix[es] a few emotional aspects into an otherwise mentally dominated list of items" (Flensborg-Madsen, Ventegodt \& Merrick 2006: 2217).

Hier wird deutlich, dass es auch kritische Stimmen und Untersuchungen bezüglich Antonovskys Konzept gibt. Grundsätzlich ist das Konzept der Salutogenese jedoch als Gesundheitsverständnis heutzutage fest verankert. Lediglich einzelne Hypothesen bezüglich der Fragebögen werden von manchen Autoren hinterfragt und z.T. widerlegt (vgl. Flensborg-Madsen, Ventegodt \& Merrick 2006).

\subsubsection{Salutogenese und Seniorensport}

„Ausgehend von einem salutogenetischen Gesundheitsverständnis, ist jeder Mensch in der Lage, durch Eigenaktivität positiv auf eigene [...] Risiken Einfluss zu nehmen“ (Wollesen 2009: 14). 
Die salutogenetische Ausgangsfrage lässt sich folgendermaßen formulieren:

„Wie kommt es, dass Menschen auch unter Bedingungen, unter denen viele erkranken, gesund bleiben?“ (Antonovsky 1997:190)

Zum einen ist die Auswahl eines gesundheitsfördernden Verhaltens notwendig und das damit eingeschlossene Erfassen und Ausbilden von ,CopingStrategien'. Zum anderen ist ein erfolgreicher Umgang mit Stressoren vonnöten, also das richtige ,Coping' zur richtigen Zeit. Beide Aspekte prägen das Kohärenzgefühl, welches wiederum die Grundeinstellung des gesundheitsorientierten Denkens fördert und dem Menschen eine positive Position auf dem Gesundheits-Krankheits-Kontinuum ermöglicht.

Außerdem kann grundsätzlich festgehalten werden, dass Menschen, die sich auf der positiven Seite des Kontinuums befinden, im Hinblick auf körperliche Betätigung eine dem Sport zugewandte Lebenseinstellung mitbringen, während Menschen mit vielen, besonders subjektiven Leiden eher als unsportlich und generell nicht an Sport interessiert einzustufen sind (vgl. Dahlhaus 2007). Ist jedoch die erste Hürde des Einstiegs in die sportliche Betätigung einmal genommen, zeichnet sich ebenfalls bei den gesundheitlich stark eingeschränkten Menschen eine positive Entwicklung ab. Daher ist es umso wichtiger, das Sporttreiben mit dem Konzept von Antonovsky in Verbindung zu setzen und die Relevanz von körperlicher Aktivität in Bezug auf ein positives Kohärenzgefühl aufzuzeigen. Eine grundsätzliche Tendenz dieses Trends findet sich in der qualitativen Erhebung im methodischen Teil dieser Arbeit wieder.

Somit ist die Metapher des ,guten Schwimmers' direkt auf die sportliche Aktivität der Seniorinnen und Senioren zu beziehen und nicht nur im übertragenen Sinne zu verstehen, sondern durchaus wörtlich zu nehmen.

Des Weiteren spielt der Aspekt der Motivation, auf welchen in einem späteren Kapitel verstärkt eingegangen wird, in Verbindung mit dem SalutogeneseKonzept eine wichtige Rolle. Gerade weil das Alter als eine „Phase der eingeschränkten Leistungsfähigkeit, als Phase, in der ein Mensch nicht mehr mit den anderen mithalten kann, als Zeitpunkt, an dem er auf dem Abstellgleis 
der Gesellschaft landet [, gilt]" (Radzik-Bolt 2006: 12), ist es nötig, genau diesem Prozess entgegenzuwirken, negativen Einflüssen vorzubeugen und jene ggf. aufzuhalten. Gelingt dies, erleben Menschen im hohen Alter eine gute Lebensqualität und weisen eine grundsätzliche Altagszufriedenheit auf (vgl. Radzik-Bolt 2006).

\subsection{Degenerative Veränderungen}

Im Laufe der Zeit und über einige Jahre hinweg verändert sich der Körper des alternden Menschen zunehmend und in den meisten Fällen negativ. Dabei gibt es neben typischen Krankheiten, wie koronarer Herzkrankheit, Schlaganfall, peripherer arterieller Durchblutungsstörung in Kombination mit Diabetes Mellitus und Hypertonie, zusätzliche Organveränderungen sowie Funktionsveränderungen. Zu den Organ- und Gewebeveränderungen gehören beispielsweise ein gekrümmtes Rückgrat, ein Zuwachs an Fettgewebe, eine Abnahme der Muskelmasse sowie des Gehirns und der Elastizität der Haut.

$\mathrm{Zu}$ den altersbedingten Funktionsveränderungen können die Abnahme des Herzzeitvolumens (weniger Blut wird vom Herzen in den Blutkreislauf gepumpt) sowie der Lungenfunktion und der eventuelle Anstieg des Blutdrucks gezählt werden (vgl. Singer 1981).

Hinzu kommen häufige Krankheiten wie zum Beispiel Arthrose oder Osteoporose (vorwiegend bei Frauen), also eine Abnahme der Knochenmasse (vgl. Meusel et al. 1980).

Des Weiteren sind das Nervensystem inklusive einer verminderten Durchblutung im Gehirn, das Immunsystem inklusive einer erhöhten Anfälligkeit in Bezug auf Krankheiten bei Nichtaktivität und die Psyche, zum Beispiel durch Verluste des Ehepartners und langjähriger Freunde betroffen (vgl. Tomasch 1997). 
Generell bleibt festzuhalten, dass der Bewegungsapparat einschließlich seiner Belastbarkeit oftmals „den limitierenden Faktor [...] dar[stellt]“ (Meusel et al. 1980: 41), was die sportliche Betätigung angeht.

Hiermit sind sowohl akute als auch chronische Krankheiten einbezogen. Somit wird erneut in Bezug auf den Teufelskreis ${ }^{1}$ deutlich, dass lediglich regelmäßige Bewegung Degenerationen vorbeugen kann und daher „Übung stärkt und Untätigkeit schwächt“ (Dahlhaus 2007: 43).

All diese degenerativen Veränderungen führen in einigen Fällen zu Einschränkungen in der Lebensqualität der älteren Menschen, da diese oftmals nicht mehr komplett selbständig agieren können und auf (kostenintensive) Hilfe angewiesen sind. Es gibt eine hohe Sturzrate von Menschen im Seniorenalter. So stürzen statistisch gesehen etwa ein Drittel der Senioren ab 65 Jahren einmal im Jahr oder häufiger, während „Frauen (40\%) häufiger stürzen als Männer (28\%)“ (Schlicht \& Schott 2013: 139). „Etwa jeder zehnte Sturz im Alter geht mit einer Fraktur und den daraus entstehenden Folgen und Kosten einher“ (Wollesen 2009: 12). Aus genau diesem Grund zeigen Krankenkassen ein erhöhtes Interesse an der Aufklärung über präventive Maßnahmen speziell für ältere Menschen in der Gesellschaft.

Die wichtigsten physiologischen Sturzrisikofaktoren im Alter sind einerseits der Muskelabbau, insbesondere in den Beinen, jedoch auch Gleichgewichtsstörungen sowie eine eingeschränkte Funktionsfähigkeit des HerzKreislauf-Systems und Beeinträchtigungen des visuellen Sinns (vgl. Wollesen 2009). Zudem können externe Sturzrisikofaktoren entstehen, welche durch Umweltbedingungen beeinflusst werden, wie beispielsweise unebenes Terrain, rutschige Oberflächen oder plötzliche Hindernisse (Schlicht \& Schott 2013).

\footnotetext{
${ }^{1}$ Vgl. hierfür auch englisches Zitat auf Seite 19
} 


\subsection{Konditionelle Fähigkeiten - Trainierbarkeit und Bedingungen im Alter}

Die konditionellen Fähigkeiten, nämlich Kraft, Ausdauer, Beweglichkeit und Schnelligkeit, können und sollten im Alter unterschiedlich gut trainiert werden. Bei allen sportlichen Betätigungen gilt der Grundsatz, dass ein gutes Aufwärmen unumgänglich ist. Auf die einzelnen konditionellen Fähigkeiten und ihre Trainierbarkeit im Alter soll in den folgenden Unterpunkten kurz und präzise eingegangen werden. Als Trainierbarkeit wird folgende Definition zur Verständnisklärung vorausgesetzt: „Trainierbarkeit wird allgemein definiert als die organismische Eigenschaft eines Menschen, auf sporttypische Trainingsbelastungen und Trainingsanforderungen zielgerichtet mit spezifischen Anpassungsprozessen physischer und psychischer Funktionssysteme zu reagieren" (Wollny 2002: 58). Vereinfacht ausgedrückt geht es bei der Trainierbarkeit des Menschen um die mögliche Spanne zwischen völlig untrainiert bis hin zu gut trainiert (vgl. Conzelmann 1997).

\subsubsection{Kraft}

Kraft beinhaltet „die Fähigkeit eines Muskels, Bewegungswiderstände zu überwinden (dynamischer Aspekt) oder äußeren Kräften entgegenzuwirken (statischer Aspekt)“ (Oschütz \& Belinová 2003: 170).

Da der Rückgang der Kraft „sich ab dem 45. bis 50. Lebensjahr bis zum 80. Lebensjahr auf ca. 10 bis 15\% pro Dekade verstärkt" (Werle, Woll \& Tittlbach 2006: 76) und ab dem 70. bis 80. Lebensjahr noch deutlicher ansteigt, wird deutlich, welche Rolle ein Krafttraining im Alter spielt (vgl. Willimczik 2009, Eichberg \& Mechling 2009).

Grundsätzlich lässt sich ein gewisser Rückgang der Kraftwerte nicht aufhalten. Dies zeigen Studien, welche belegen, dass die Gesamtmuskelmasse in 
Kilogramm von durchschnittlich etwa 38-40 kg abnimmt auf ca. $26 \mathrm{~kg}$ bei einem 70-jährigen Menschen (vgl. Schmidtbleicher 2009). Zwar weisen sportlich trainierte Senioren höhere Maximalwerte auf, doch unterliegt „die Abnahme der Kraft [...] in gleichem Maße dem Altersprozess [...] wie bei inaktiven Personen" (Eichberg \& Mechling 2009: 344-345). Dies ist in den meisten Fällen sowohl auf die grundlegende Verkleinerung des Muskelquerschnitts als auch auf eine degenerierte Koordinations- und Innervationsfähigkeit zurückzuführen (vgl. Stemper 2001).

Die konditionelle Fähigkeit der Kraft ist im höheren Alter generell gut zu trainieren - zum einen, um einer weiteren Verschlechterung der Kraftwerte präventiv entgegenzuwirken und zum anderen, um eben diese teilweise sogar zu erhöhen (vgl. Stemper 2001). Dies wird deutlich am messbaren Zuwachs von Kraft, wie in einigen Trainingsstudien belegt (vgl. Werle, Woll \& Tittlbach 2006). Der hierbei wichtigste Punkt ist die Dosierung der Gewichte, sodass eine so genannte Pressatmung vermieden wird. Dieses heißt, dass das Gerätetraining moderat gewählt werden sollte und Gewichte, die an die Maximalkraft grenzen, ausgeschlossen werden müssen (vgl. Singer 1981).

In einer Studie, welche mit 20 Frauen (über 50) mithilfe von Übungen mit dem Theraband durchgeführt wurde, ergaben Messungen von Delshad et al. (2013) ebenfalls einen Zuwachs der Kraftwerte sowie gleichzeitig einen reduzierten Körperfettanteil. Dies zeigt, dass nicht ausschließlich Gerätetraining in Fitnessstudios wirksame Effekte erzielen kann, sondern auch freie Übungen mit beispielsweise Therabändern oder Hanteln. Lediglich die Gefahr, Übungen falsch auszuführen, ist beim Training ohne geführte Geräte größer, sodass zum einen viel geübt werden muss, ein gutes eigenes Körpergefühl zu entwickeln und zum anderen auf Seiten der Übungsleiter eine gute Betreuung und Korrektur gegeben sein muss.

Darüber hinaus wurde in weiteren Studien der Zusammenhang von Krafttraining und Gleichgewichtsfähigkeit untersucht. Als Beispiele können die Studien von Lee \& Park (2013) sowie von Joshua et al. (2014) genannt werden. In der 
Studie von Lee \& Park (2013) wird die Hypothese aufgestellt, dass ein Rückgang der Muskelmasse der unteren Extremitäten zu einem erhöhten Risiko des Stürzens und des sitzenden Lebensstils (,sedentary lifestyle') führe. Um dies zu belegen, führten sie ein Krafttraining der unteren Extremitäten mit 50 Probanden im Alter von 65-82 Jahren durch. Hier wurde festgestellt, dass durch die Kräftigung eine niedrigere Sturzrate erreicht wurde und somit ein Krafttraining durchaus positiven Einfluss auf die Gleichgewichtsfähigkeit bei älteren Menschen hat.

Gleiche Schlussfolgerungen wurden auch in der Studie von Joshua et al. (2014) gezogen: Ein spezifisches Krafttraining der unteren Extremitäten bewirkt sogar noch bessere Resultate als ein herkömmliches Gleichgewichtstraining. So kann ein ausgewähltes Krafttraining demnach nicht nur zu einem Kraftzuwachs in der Muskulatur beitragen, sondern ebenfalls einen Rückgang des Sturzpotenzials zur Folge haben.

\subsubsection{Ausdauer}

Die Ausdauer ist eine „komplexe motorisch-konditionelle Fähigkeit, einer sportlichen Belastung physisch und psychisch möglichst lange widerstehen zu können und sich nach sportlichen Belastungen möglichst rasch zu erholen“ (Oschütz \& Belinová 2003: 156, zit. nach Grosser et al. 1993: 97).

Die allgemeine aerobe dynamische Ausdauer ist die geeignetste Form für den Seniorensport, da sie sportartunspezifisch trainiert werden kann. Da die durchschnittliche Abnahme der Ausdauerleistungsfähigkeit ab dem 50. Lebensjahr etwa $10 \%$ bis $15 \%$ pro Lebensdekade entspricht, ist auch dieses Training ein wichtiger Baustein im Alterssport (vgl. Werle, Woll \& Tittlbach 2006, Willimczik 2009, Eichberg \& Mechling 2009). Im Alter von 60 Jahren verzeichnen Männer bereits einen Verlust ihrer maximalen Sauerstoffaufnahme von 25-33\%, während Frauen mit 20-25\% etwas unter den Werten der Männer einzuordnen sind (vgl. Conzelmann \& Blank 2009). 
Trainierte Senioren zeigen zwar deutlich höhere Gesamtwerte in puncto Ausdauerleistungsfähigkeit, verzeichnen jedoch ebenso einen Abfall mit erhöhtem Lebensalter, welcher im Vergleich zu Untrainierten als nahezu parallel laufend anzusiedeln ist (vgl. Eichberg \& Mechling 2009). Die Ursachen für den Rückgang liegen im physiologischen Bereich: Das zu erreichende Herzschlagvolumen ist im Alter geringer sowie die Herzfrequenz im Allgemeinen (vgl. Stemper 2001). Der oftmals angenommene Mythos, dass sportlicher Leistungsabfall genetisch bedingt sei, wird durch physiologische Tests und deren Messdaten widerlegt, welche Auskunft über die sportliche Aktivität der Probanden geben (vgl. Conzelmann \& Blank 2009).

Ein wohlüberlegtes, dem Alter angemessenes Ausdauertraining sorgt im Idealfall für ein „ökonomischer arbeitendes Herz, eine bessere Sauerstoffversorgung des gesamten Organismus und eine höhere Resistenz gegenüber pathologischen Veränderungen des Herz-Kreislauf-Systems“ (Oschütz \& Belinová 2003: 159-160). In bereits vorliegenden Studien zur Trainierbarkeit der Ausdauer im höheren Alter lassen sich Verbesserungen der Ausdauerleistungsfähigkeit von durchschnittlich bis zu 22\% feststellen (vgl. Eichberg \& Mechling 2009). [Hierbei handelt es sich um kurz- und langfristige Interventionen von 16 Wochen bis 12 Monaten.]

Dabei sollte immer ausreichend Zeit zur Regeneration zwischen den Phasen der Belastung und Entlastung berücksichtigt werden, außerdem sollte das Training den Ansprüchen des individuellen Sportlers gerecht werden.

Ein grundsätzlicher Vorteil beim Ausdauertraining ist der potenzielle Einsatz von Pulsuhren, mit deren Hilfe die Herzfrequenz stets beobachtet werden kann und das Erreichen zu hoher Frequenzen vermeidbar wird. 


\subsubsection{Beweglichkeit}

Die Beweglichkeit ist die „Fähigkeit des Menschen, Bewegungen mit großer Amplitude selbst oder mit unterstützendem Einfluß äußerer Kräfte in einem oder mehreren Gelenken auszuführen“ (Oschütz \& Belinová 2003: 182).

Zudem ist die Beweglichkeit abhängig von der "Gelenkstruktur der Dehnfähigkeit, der Elastizität, dem Tonus, dem Umfang und der Länge der Muskeln, der Anordnung der Sehnen und Bänder und der Dehnfähigkeit der Sehnen, Gelenkkapseln und der Haut" (Willimczik 2009: 341).

Da die Beweglichkeit bereits ab dem 25. Lebensjahr bei Nichtaktivität nachlässt, ist regelmäßiges Dehnen zur Erhaltung der Beweglichkeit bis ins hohe Alter unumgänglich. Durch Studien lässt sich feststellen, dass die Beweglichkeit im Seniorenalter bei entsprechendem Training mindestens zu stabilisieren, wenn nicht zu verbessern ist (vgl. Werle, Woll \& Tittlbach 2006). Darüber hinaus gilt als belegt, dass die Beweglichkeit bei Frauen zwar grundsätzlich höhere Verluste einzubüßen scheint, Frauen durchschnittlich dennoch beweglicher sind als Männer (vgl. Eichberg \& Mechling 2009).

Für die Bewegungsausführung an sich sollte sich das Halten der Dehnung mit der Zeit steigern genau wie die Dynamik der Dehnung selbst (vgl. Denk, Pache \& Schaller 2003). Einige Gelenke scheinen sich leichter bezüglich erhöhter Beweglichkeit trainieren zu lassen, wie zum Beispiel die Wirbelsäule und das Hüftgelenk, während bei Knie- und Fußgelenken in Untersuchungen geringere Verbesserungen der Beweglichkeit gemessen werden (vgl. Eichberg \& Mechling 2009).

\subsubsection{Schnelligkeit}

Schnelligkeit im Sport ist die „Fähigkeit, aufgrund kognitiver Prozesse, maximaler Willenskraft und der Funktionalität des Nerv-Muskel-Systems 
höchstmögliche Reaktions- und Bewegungsgeschwindigkeiten unter bestimmten gegebenen Bedingungen zu erzielen“ (Oschütz \& Belinová 2003: 190, zit. nach Grosser 1991: 315).

Grundsätzlich kann festgehalten werden, dass lediglich die Handlungsschnelligkeit bzw. Reaktionsschnelligkeit im Altag eine wichtige Rolle für Senioren spielt. Andere Formen der Schnelligkeit, wie beispielsweise die Sprintschnelligkeit, stehen im Alterssport nicht mehr im Vordergrund wegen der hohen Verletzungsgefahr, die bei schnellkräftigen Bewegungen vorherrscht.

Da die Reaktionsschnelligkeit im Alter abnimmt (etwa $13 \%$ in den Jahren von 20 bis 60 ), sind Seniorinnen und Senioren, gerade in gemischten Gruppen, in denen einige jüngere Teilnehmer anwesend sind, oftmals frustriert, weil sie trotz enormer Willensstärke nicht mehr die Zeiten und Reaktionen, von früher' erreichen können (vgl. Willimczik 2009). Hier ist eine hohe Sensibilität auf Seiten der Übungsleiter gefragt, um großen Enttäuschungen vorzubeugen.

Des Weiteren spielt die Reaktionsschnelligkeit eine wichtige Rolle, wenn es darum geht, Stürzen oder unvorhergesehenen Geschehnissen im Alltag vorzubeugen und/oder ihnen schnell handelnd gegenüberzutreten (vgl. Schmidtbleicher 2009).

\subsection{Koordinative Fähigkeiten - Trainierbarkeit und Bedingungen im Alter}

Als Voraussetzung zur Entwicklung der so genannten koordinativen Fähigkeiten bedarf es zunächst einer begrifflichen Erklärung der motorischen Entwicklung. Bei der motorischen Entwicklung handelt es sich um "eine Reihe von miteinander zusammenhängenden, auf den motorischen Persönlichkeitsbereich bezogenen Veränderungen, die bestimmten Orten eines zeitlichen Kontinuums eines individuellen Lebenslaufs, vorzugsweise über das kalendarische Alter, zuzuordnen sind“" (Willimczik 2009: 21). Die motorische Trainierbarkeit erweist 
sich ab dem 30. Lebensjahr als aufwendiger im Vergleich zu Übungen in früheren Jahren (vgl. Carius 2012).

Im Verlauf der motorischen Entwicklung verändern sich zudem die konditionellen und koordinativen Fähigkeiten. Die koordinativen Fähigkeiten sind die entscheidenden Parameter, welche für die Qualität einer Bewegungsregulation und -ausführung mitbestimmend sind (vgl. Denk, Pache \& Schaller 2003).

Zusätzlich lassen sich die koordinativen Fähigkeiten nach Hirtz (1994) in weitere Subkategorien unterteilen:

- Gleichgewichtsfähigkeit

- Antizipationsfähigkeit

- Kienästhetische Differenzierungsfähigkeit

- Kopplungsfähigkeit

- Orientierungsfähigkeit

- Reaktionsfähigkeit

- Rhythmusfähigkeit

- Umstellungsfähigkeit

Die Wichtigkeit der einzelnen koordinativen Fähigkeiten kristallisiert sich bereits im Alltag heraus: So sorgt ein gutes Gleichgewichtsvermögen beispielsweise für eine Prävention vor Stürzen; Alltagsfertigkeiten, wie zum Beispiel das Aufstehen aus dem Bett oder das Überqueren von Straßen, können besser bewältigt werden und durch das erfolgreichere Durchführen von Mehrfachhandlungen steigert sich das persönliche Wohlbefinden und Selbstwertgefühl (vgl. Schaller \& Wernz 2000). Grundsätzlich muss die Rolle der Gleichgewichtsfähigkeit insbesondere bei Seniorinnen und Senioren mit Vorsicht behandelt werden, da im höheren Alter einige Faktoren hinzukommen, welche die Gleichgewichtsfähigkeit möglicherweise einschränken. Ein Risikofaktor für Stürze im Alter ist beispielsweise eingeschränktes Sehen: Die Gleichgewichtsfähigkeit ist bei schlechter Sehkraft deutlich beeinträchtigt (vgl. 
Rao 2005). Die niedrige Reaktionszeit, welche in vielen Fällen zu Stürzen führt, ist oftmals gekoppelt mit Einschränkungen des visuellen Sinns. Um diese Hypothese zu bestätigen, untersuchten Reed-Jones et al. (2012) mithilfe von drei verschiedenen Trainingsgruppen, welchen Effekt visuelles Training im Vergleich mit herkömmlichem Gleichgewichts- oder Fitnesstraining bewirkt. Die Gruppe, welche das visuelle Training (,visual training') durchführte, übte mithilfe einer Nintendo® Wii Fit Plus auf einem Balance-Board durch Gewichtsverlagerung störenden Gegenständen auszuweichen (,obstacle avoidance ), welche auf dem Bildschirm erschienen.

Im abschließenden finalen Test nach 12 Wochen führte die visuelle Trainingsgruppe die Aufgabe, möglichst schnell und fehlerfrei durch einen Hindernisparcours zu gehen, am besten durch. Hier wird deutlich, wie viel Einfluss der visuelle Sinn auf die Gleichgewichtsfähigkeit hat und wie eng er mit der Thematik des Stürzens im höheren Alter verwandt ist.

Spätestens ab dem 50. Lebensjahr kommt es zu verstärkten Einbußen im Bereich der koordinativen Fähigkeiten, wird diesen nicht mit entsprechendem Training entgegengewirkt (vgl. Roth \& Roth 2009). Nichtsdestotrotz existieren wenige Forschungsarbeiten zur Thematik der Trainierbarkeit der motorischen Fähigkeiten, insbesondere im späteren Erwachsenenalter (vgl. Willimczik 2009).

Ein Forschungsfeld, welches insbesondere in den letzten Jahren zunehmend an Bedeutung gewonnen hat, ist die Untersuchung des Bewegungsverhaltens nach Schlaganfällen (vgl. u.a. DeFina et al. 2013). Speziell die Koordination und das Gleichgewichtsvermögen sind nach Schlaganfällen beeinträchtigt, sodass in vielen Fällen bereits das Gehen über eine bestimmte Distanz schwerfällt (vgl. Gordon, Wilks \& Mc Caw-Binns 2013). Zur beispielhaften Verdeutlichung der Forschungsarbeiten bezüglich dieser Thematik werden zwei Studien kurz vorgestellt: In einer Arbeit über aerobes Gehen in Verbindung mit einem Zuwachs an Lebensqualität untersuchen Gordon, Wilks \& Mc Caw-Binns (2013) den Effekt von aerobem Gehtraining im Vergleich zu Massagen der unteren Extremitäten. Zur Erfassung der subjektiven gesundheitlichen Befindlichkeit 
mussten die Probanden einen Fragebogen beantworten und der Fitnesszustand wurde zudem mit einem 6-minütigen Ausdauertest gemessen. Als Resultat kommen die Autoren zu dem Schluss: „Aerobic walking improves the physical health component qualitiy of life and endurance in persons with chronic stroke." (1180). Laut der Autoren hilft demnach ein moderates Ausdauertraining nach Schlaganfällen, eine verbesserte subjektive Lebensqualität zu erreichen.

Darüber hinaus untersuchten Tang, Eng \& Rand (2012) den Zusammenhang von gefühlter und gemessener Veränderung im Bewegungsverhalten nach Schlaganfällen. Sie führten einen so genannten 6-minute walk test (6 MWT) mit 22 Probanden vor Beginn und im Anschluss an eine 3-monatige Trainingsintervention durch. Die eine Untersuchung beinhaltet die absolvierte Distanz, welche in den sechs Minuten zurückgelegt wurde und die andere Untersuchung beleuchtet die subjektive Wahrnehmung der Veränderung der Leistungsfähigkeit der Individuen auf einer 15-Punkte-Likert-Skala (,deutlich verschlechtert' über ,keine Veränderung' bis hin zu ,deutlich verbessert'). Bei niedrig erreichten Distanzmessungen wird auch subjektiv keine große Veränderung wahrgenommen, während bei weiter zurückgelegten Strecken automatisch eine subjektive Verbesserung assoziiert wird, auch wenn bereits im Pretest weite Strecken absolviert wurden. Hier zeichnet sich ab, dass das subjektive Wohlbefinden zunimmt, wenn weitere Strecken zurückgelegt werden können und implizit eine größere Selbständigkeit erreicht wird im Anschluss an einen Schlaganfall.

Der Rückgang der koordinativen Fähigkeiten hängt (abgesehen von akuten Schicksalsschlägen) hauptsächlich ab von Schwächungen der Sinnesorgane, Muskulatur und Gelenkbeweglichkeit. Jedoch sollte ebenfalls eine mangelnde Vorerfahrung im früheren Kindes- und Jugendalter nicht unterschätzt werden (vgl. Schaller \& Wernz 20103). Diese Thematik wird im empirischen Teil dieser Arbeit aufgegriffen und stärker beleuchtet werden. Im Folgenden werden die Bedeutungen des Trainings der koordinativen Fähigkeiten (besonders) im Seniorenalter zusammengefasst: 
- „Kraft-, Schnelligkeits- und Ausdauerdefizite werden bis zu einem gewissen Grad kompensiert.

- Komplizierte Fertigkeiten werden sicher ausgeführt.

- Bewegungsfertigkeiten werden schneller erlernt.

- Bewegungsfertigkeiten werden zweckentsprechender eingesetzt.

- Bewegungsfertigkeiten werden bei Situationsänderungen zweckmäßiger variiert.

- Die Umstellung auf ungewohnte Bedingungen gelingt leichter.

- Durch schnelles Reagieren auf Stolpern und Ausrutschen wird das Verletzungsrisiko gemindert.

- Die Ermüdung setzt später ein.

- Ein gutes Niveau koordinativer Fähigkeiten steigert die Freude an sportlicher Betätigung“ (Schaller \& Wernz 2010²: 24).

\section{$4 \quad$ Motive und Motivation}

„Die Motivation zur körperlich-sportlichen Aktivität ist ein lebenslanger Prozess, d.h. sie muss - vom ersten Kindersport bis zur Seniorengymnastik - immer wieder neu hergestellt und aufrechterhalten werden“ (Fuchs 2003: 237).

\subsection{Begrifflichkeiten}

Um die unterschiedlichen Gründe darlegen zu können, warum Seniorinnen und Senioren bis ins hohe Alter Sport treiben sollten, bedarf es zunächst einer Klärung der Begrifflichkeiten Motive versus Motivation.

Motive werden laut Heckhausen (1989) als „überdauernde und relativ konstante Wertungsdispositionen, die sich auf definierte Inhaltsklassen von Handlungszielen beziehen" (Fuchs 1997: 22), bezeichnet und müssen stets in gewissen Umständen aktiviert werden, um das Verhalten des Individuums zu 
beeinflussen. Die von Denk \& Pache (2003) beschriebene Definition des Motivs als „eine [...] relativ überdauernde latente Verhaltensdisposition“ (76) deckt sich weitestgehend mit der zuvor genannten Begriffsbeschreibung. Außerdem ist das Motiv ein "hypothetisches Konstrukt“ (Gabler 2002: 14), da man es nicht direkt beobachten kann, sondern es vielmehr für Verständnis der Handlungen bestimmter Individuen sorgt (vgl. Rheinberg 2002).

Die Motivation hingegen wird als „die akute Verhaltensbereitschaft [bezeichnet], während das Motiv die im Hintergrund wirkende chronische Verhaltenspräferenz darstellt“ (Fuchs 1997: 22). Resultierend aus Motiv und Situation ergibt sich die Motivation, spezifisch zu handeln. Es geht hierbei also um eine Art Wechselwirkung zwischen Situationen und Motiven. Bei Denk \& Pache (2003) ist die Motivation als „[die] durch situative Faktoren aktivierte [...] Handlungsbereitschaft" (76) gekennzeichnet. Die Motivation führt also zu der Klärung beziehungsweise Antwort, warum Menschen im hohen Alter Sport treiben. Einfacher ausgedrückt heißt das: Ist eine ausreichende Motivation vorhanden, führt diese zur Sportpartizipation (vgl. Dahlhaus 2007). Der Begriff der Motivation kommt ursprünglich aus dem Lateinischen (,movere = bewegen, in Bewegung setzen" (Gabler 2002: 12)) und beschreibt daher im übertragenen Sinne, was Menschen dazu bewegt, Sport zu treiben.

Grundsätzlich sollte jedoch beachtet werden, dass es sich bei der Motivation stets um einen abstrakten Begriff handelt, welcher nicht als universell und für alle Lebenssituationen gleich zu behandeln ist. Im allgemeinen Sinne geht es idealerweise dennoch darum, dass eine Person „(1) ein Ziel hat, daß [sie] (2) sich anstrengt und daß [sie] (3) ablenkungsfrei bei der Sache bleibt" (Rheinberg 2002: 16). Sind die letzten beiden Punkte in der Ausführung eingeschränkt bzw. nicht gegeben, kommt es in den meisten Fällen zu Misserfolgen und das eigentliche Ursprungsziel kann lediglich unzureichend oder gar nicht erreicht werden. 
Des Weiteren wird unterschieden zwischen extrinsischer und intrinsischer Motivation. Während die extrinsische Motivation Ziele über die eigentliche Zielintention hinaus beschreibt (in dieser Arbeit z.B. über das Sporttreiben hinaus, wie etwa im sozialen oder gesundheitlichen Bereich), befasst sich die intrinsische Motivation mit der Durchführung oder dem Erreichen der Handlung selbst (vgl. u.a. Gabler 2002, Zarotis 1999 und Vallerand \& Loisier 1999). Resultiert das Sporttreiben also aus der intrinsischen Motivation, wird die Handlung „um [ihrer] Selbstwillen“ vollzogen (Gabler 2002: 160). Das Ziel der Tätigkeit ist demnach nicht ein vorab definierter Endzustand, sondern vielmehr das intensive Betreiben einer Handlung, „weil der Verrichtungszustand in sich hoch belohnend ist" (Rheinberg 2006: 332). Bei der intrinsischen Motivation handelt es sich daher eher um das Erlebnis des Prozesses an sich inklusive der Gefühle, welche alle Sinne aufnehmen können, und eher um das vermittelte Wissen als um das Ergebnis, welches am Ende aus der Betätigung resultiert (vgl. Lapointe \& Perreault 2013).

Bei der extrinsischen Motivation stimmen Ziel und Zweck der Handlung nicht unbedingt überein, sondern die Intentionen gehen weit über das eigentliche Ziel, in diesem Fall des Sporttreibens, hinaus. Oftmals sind die Ziele der extrinsischen Motivation monetärer Art, wie beispielsweise ein Preisgeld oder einen Pokal zu erhalten, doch im Gesundheitssport handelt es sich bei den Zielen eher um eine langfristige Verbesserung des Wohlbefindens (vgl. Lapointe \& Perreault 2013). Um etwaige Missverständnisse in der Begriffszuweisung zu vermeiden, beschreibt Rheinberg (2006) die intrinsische Motivation als tätigkeitszentriert und die extrinsische Motivation als zweckzentriert. Des Weiteren sollte nicht ausgeschlossen werden, dass sich beide Arten von Motivation überlappen bzw. die ursprüngliche Motivation umschwenkt in eine andere: „So könne eine Tätigkeit durchaus aufgrund extrinsischer Motive gestartet werden, dann aber beim Tätigkeitsvollzug aufgrund intrinsischer Anreize weitergeführt werden" (Rheinberg 2006: 333).

Dieses Beispiel lässt sich auf beliebige Situationen im Sportbereich übertragen. Wenn eine Person beispielsweise auf Anraten eines Arztes mit dem Sporttreiben beginnt, mag diese anfangs sehr skeptisch sein und im Vorfeld 
keine Freude bei dem Gedanken an die Aktivität empfinden. Entpuppt sich diese Aktivität jedoch im Verlauf der Durchführung als positiv und gemütssteigernd, so ändert sich die Art der Motivation für das zukünftige Ausüben dieser Tätigkeit (vgl. Sebire, Standage \& Vansteenkiste 2009).

Bezogen auf die gegenwärtige Motivationspsychologie ist als entscheidend hinzuzufügen, dass diese bestimmte Verhaltensweisen oder gewisse Tätigkeiten „weder aus Merkmalen der Person (Triebe, Instinkte etc.) noch allein aus Merkmalen der Situation (Reize, objektive Stimuli etc.) zu verstehen versucht" (Rheinberg 2002: 44). Die folgenden Ansätze können also nicht per se als isoliert betrachtet, sondern in vielen Fällen als ergänzend angesehen werden.

Laut Gabler (2002), welcher sich sprachlich von extrinsischer und intrinsischer Motivation distanziert, dominieren sechs verschiedene Ansätze die Erklärung eines motivierten Verhaltens: Der biologisch-physiologische Ansatz beschreibt das eigenständige Regulieren des Organismus, also eine Art Befriedigung der Grundbedürfnisse eines jeden einzelnen Menschen. Der ethologisch-instinkttheoretische Ansatz reguliert eine festgelegte Definition eines Instinktes und überträgt diesen auf das jeweilige Motivationsgebiet (z.B. Sport). Der tiefenpsychologisch-triebtheoretische Ansatz beschreibt ein Verhalten, welches insbesondere durch „triebhafte Impulse beeinflusst [wird], deren Herkunft im Unbewussten verborgen bleibt" (Gabler 2002: 40). Hierzu gehört etwa der Aggressionsabbau mittels Sporttreiben. Der behavioristisch-lerntheoretische Ansatz basiert auf Erfahrungen und Gewohnheiten. Im persönlichkeitstheoretischen Ansatz legt das Individuum seine Ziele selbst fest, indem es beispielsweise Leistung oder Geselligkeit anstrebt. Der kognitiv-handlungstheoretische Ansatz beschreibt den Menschen als "handelndes Wesen" (Gabler 2002: 44), welches Ziele vor Augen hat und diese mithilfe von Handlungen erreicht. Die getroffenen Entscheidungen sind individuell zu verantworten und zu reflektieren (vgl. Gabler 2000). 
Grundsätzlich kann festgehalten werden, dass jeder Sportler und jede Sportlerin bestimmte Absichten verfolgen, also gewisse Intentionen haben, um ihre Ziele zu verwirklichen. Dies geschieht allerdings nie ohne eine „aktive [...] Auseinandersetzung mit der Umwelt“ (Nitsch 2000: 55), welche immer in Wechselwirkung zwischen einer Situation und einem Individuum steht (vgl. Rheinberg 2002).

\subsection{Modelle und Theorien}

Zur exakteren Erläuterung der bisher aufgeführten verschiedenen Motivationstheorien gibt es eine Vielzahl von Modellen, von denen im Folgenden eine Auswahl kurz beschrieben wird.

Eines der frühesten Modelle, das Health-Belief-Modell (HBM) von Rosenstock (1974), beschreibt die Bereitschaft von Personen, ein gesundheitsförderliches Verhalten zu erlernen, wenn bereits eine akute Gefahr einer Erkrankung besteht (vgl. Pfeffer 2010). Wird also eine Art Bedrohung oder Angst in Form von einer Erkrankung bzw. gesundheitlicher Einschränkung empfunden, müssen gewisse Barrieren überwunden werden, um positive Resultate erzielen zu können: „Je höher die eigene Anfälligkeit für eine Erkrankung eingeschätzt wird und je höher die Barrieren für die Ausführung einer Sportaktivität beurteilt werden desto weniger sind die betreffenden Personen körperlich aktiv" (Pfeffer 2010: 16). Sind die Barrieren jedoch einmal überwunden und erscheinen positive Resultate in Form von einem verbesserten Gesundheitszustand, ist das gesundheitsförderliche Verhalten erlernt und als notwendig und helfend anerkannt.

Dieses heutzutage nicht mehr als aktuell angesehene Modell wurde von weiteren Folgemodellen überholt, wie beispielsweise die Theorie der Schutzmotivation $($ PMT $=$ Protection Motivation Theory) von Rogers (1975 \& 1985). Hierbei geht es um zwei parallel ablaufende Prozesse; zum einen um die subjektive Einschätzung, inwieweit die Gesundheit als bedroht angesehen wird 
und zum anderen um die subjektive Einschätzung, inwiefern eigene Fähigkeiten zur Bewältigung dieser Bedrohung vorherrschen (vgl. Pfeffer 2010). Ein entscheidender Faktor ist demnach die eigene Fähigkeitswahrnehmung, insbesondere mögliche Schutzhandlungen zur Bekämpfung bedrohlicher Situationen. Auch in diesem Modell ist jede Verhaltensweise von Individuum zu Individuum unterschiedlich, je nachdem, wie eine Situation und die dazugehörige eigene Fähigkeit wahrgenommen werden.

Eine weitere der früh entstandenen Theorien ist die so genannte SozialKognitive Theorie nach Bandura (1977 \& 1986). Diese besagt, dass das Verhalten abhängig von Beurteilungen der individuellen Fähigkeiten und Fertigkeiten inklusive der Erwartungen an diese ist. Dadurch entstehen unterschiedliche Zielsetzungen und verschiedene Ebenen des Erreichens dieser Ziele. Die Theorie ist gekennzeichnet durch zwei Konstrukte - zum einen die Selbstwirksamkeit, also die „Überzeugung, ein bestimmtes Verhalten ausführen zu können" und zum anderen die Konsequenzerwartungen, also die „Einschätzung darüber, zu welchem Ergebnis und welchen Konsequenzen ein bestimmtes Verhalten führt" (Pfeffer 2010: 20).

Ferner existiert das so genannte Konzept der Selbstmotivation nach Dishman (1980). Hierbei ist am wichtigsten, dass die Persönlichkeitsmerkmale der Sportler Einfluss auf die eigentliche Motivation zur sportlichen Betätigung haben. Daher werden Entscheidungen, die als beschlossen feststehen, generell nicht zurückgenommen, da die Motivation an sich stärker ist als der bekannte ,innere Schweinehund”: „Je höher die Selbstmotivation, so die Leithypothese der meisten Studien, um so größer die Wahrscheinlichkeit, daß ein Sportprogramm oder eine selbst-organisierte Sportaktivität über längere Zeit aufrechterhalten wird" (Fuchs 1997: 18). Hier wird deutlich, dass die Selbstmotivation der intrinsischen Motivation sehr nah ist.

Ein weiteres Modell zur Begründung von Motivation ist das ,Sensation Seeking' nach Zuckermann (1983), welches aus vier Dimensionen besteht, in denen es jedoch lediglich kleinere Abweichungen gibt. In der ersten Dimension geht es 
um die "Suche nach Spannung und Abenteuer" (Fuchs 1997: 14), also um den Wunsch nach Risiko, gepaart mit Abenteuerlust. Die zweite Dimension beschreibt die "Suche nach neuen Erfahrungen“ (Fuchs 1997: 14), also den Wunsch nach Abwechslung und Neuerleben. In der dritten Dimension geht es um die „Enthemmung“ (Fuchs 1997: 14), also das losgelöste Auftreten des Individuums ohne jegliches Schamgefühl oder übertriebene Zurückhaltung. Die vierte und letzte Dimension ist mit „Anfälligkeit für Langeweile“ (Fuchs 1997: 14) benannt und beschreibt damit, ähnlich wie die zweite Dimension, den Wunsch nach Abwechslung und Beschäftigung. In diesem Modell spielt also in allen vier Dimensionen der Wunsch nach Risiko und Abenteuer eine wichtige Rolle, was die Motivation angeht.

Das wohl früheste ausführliche Modell der motivationalen Handlungsphasen ist das so genannte Rubikon-Modell nach Heckhausen (1989).

„Die entscheidende Innovation des Rubikon-Modells liegt in der Identifikation der diskreten Wechsel zwischen motivationalen und volitionalen Handlungsphasen [...]" (Achtziger \& Gollwitzer 2006: 278). Es betrachtet nicht nur die individuelle Zielsetzung einer Person (hier als Motivation beschrieben), sondern behandelt anschließend gleichermaßen den (erhofften) Prozess des Erreichens dieser Zielsetzung (im Folgenden als Volition beschrieben).

Das Rubikon-Modell, im Allgemeinen auch als strukturfunktionelles Modell bezeichnet, besteht aus vier aufeinander folgenden Handlungsphasen (vgl. Achtziger \& Gollwitzer 2006). Die prädezisionale Motivationsphase beschreibt den Prozess des Abwägens der Wünsche seitens der Akteure. Hier entstehen ggf. mehrere Handlungsalternativen (vgl. Backmann, Fröhlich \& Elbe 2009). Den Abschluss dieser Phase bildet eine genau definierte Zielabsicht, bildlich gesprochen die ,Überquerung des Rubikon', die in die nächste Phase übergeht. Nachdem eine individuelle Intention gebildet wurde, wird in der präaktionalen Volitionsphase überlegt, wie das Geplante realisiert werden kann, indem aktuelle Gegebenheiten beobachtet und ausgewertet werden. Anschließend, nach erfolgreicher Beobachtung und Abwägung, herrscht in der aktionalen 
Volitionsphase eine Initiierung der Handlung. Ist die Handlung erfolgreich abgeschlossen, wird in der postaktionalen Motivationsphase auf die Handlung in Verbindung mit dem Ziel zurückgeblickt und sie analysiert. Fällt die Bewertung des Ergebnisses positiv aus, ist das Ziel erreicht, wird deaktiviert und neue Ziele können gewählt werden. Fällt die Bewertung des Ergebnisses allerdings negativ aus, müssen neue oder im Niveau gesenkte Handlungen geplant werden, die zum gewünschten Resultat führen (vgl. Achtziger \& Gollwitzer 2006).

Ist dieser gedankliche Entscheidungsschritt jedoch misslungen, wird das Ziel weiterhin als aktiviert angesehen (vgl. Beckmann, Fröhlich \& Elbe 2009). In Phase 1 und 4 des Rubikon-Modells ist die Motivation aktiv, in Phase 2 und 3 die Volition (vgl. u.a. Heckhausen \& Heckhausen 2006, Gabler 2002, Achtziger \& Gollwitzer 2006).

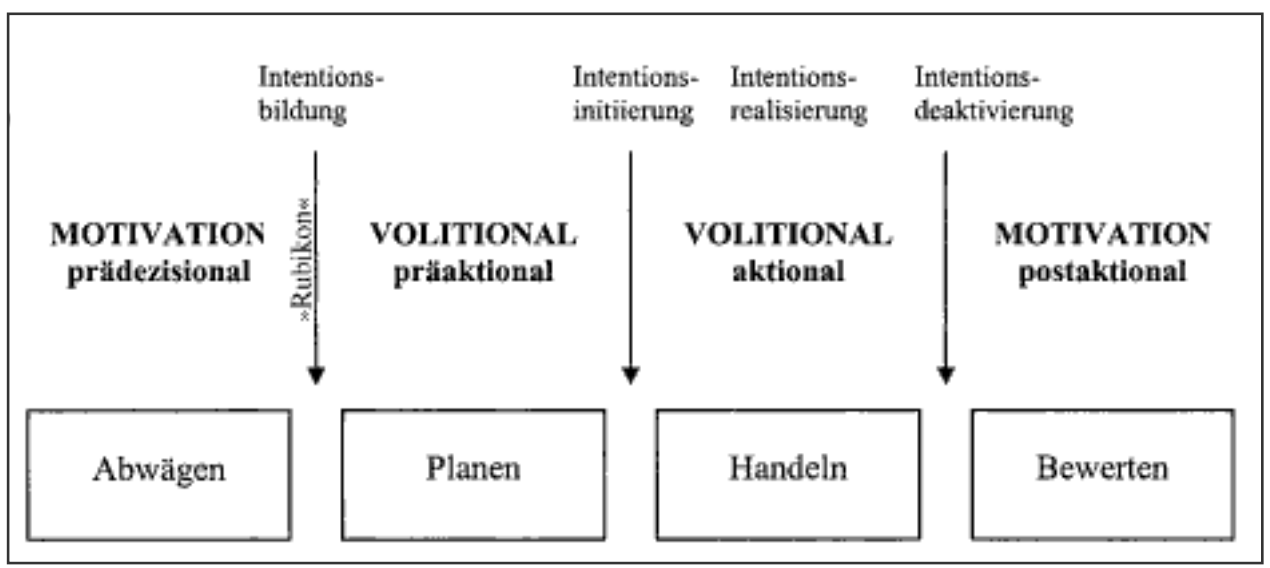

Abbildung 2: Rubikon-Modell nach Heckhausen 1989 (Sudeck 2007: 46)

Laut Beckmann, Fröhlich und Elbe (2009) ist das Rubikon-Modell von Heckhausen (1989) „gut geeignet [...], vielfältige sportliche Handlungen zu erklären“ (547). Übertragen auf eine sportliche Betätigung könnte in der ersten Handlungsphase beispielsweise der Vorsatz bzw. das Ziel lauten, gesünder leben zu wollen. Nun überlegt sich eine Person z.B. in der zweiten Handlungsphase regelmäßig Sport treiben zu wollen. Um die Intention zu 
realisieren, müsste die Person jetzt selbst aktiv werden, also beispielsweise sich eine Sportgruppe suchen, einem Verein beitreten oder individuell feste Termine für sportliche Aktivitäten bestimmen. Klappt dies ohne Einwände, wird das ursprüngliche Ziel als erreicht anerkannt, da nun regelmäßig Sport getrieben wird. Klappt dies lediglich mit Einschränkungen oder gar nicht, müssen entweder die Ansprüche gesenkt werden, also die regelmäßige sportliche Tätigkeit auf wenige Male pro Woche reduziert werden, oder aber komplett neue Strategien entwickelt werden.

Da die Zielbildung einen zentralen Punkt in der Motivationsforschung darstellt, soll auf diese Thematik nun etwas genauer eingegangen werden. Grundsätzlich werden für die Erreichung unterschiedlicher Ziele auch unterschiedliche Verhaltensweisen nachgewiesen (vgl. Hepler et al. 2012). So behaupten Hepler et al. (2012) in ihrer Studie zum Thema Selbstkontrolle, dass Ziele für Untätigkeit eher Selbstkontrolle hervorrufen, während Ziele für Aktivität oder Handlung eher impulsives Verhalten evozieren. Mit Untätigkeit ist hier beispielsweise Verzicht oder Vernunft in gewissen Situationen (z.B. Diäten) gemeint. Sie belegen in ihrer Veröffentlichung, dass eine verzichtorientierte Motivation Selbstkontrolle vereinfacht, wohingegen eine handlungsorientierte Motivation Selbstkontrolle hemmt. Dies impliziert jedoch nicht automatisch, dass jede Handlung abgebrochen wird, weil die eigene Selbstkontrolle nicht wirksam genug aktiviert ist. Es bedeutet lediglich, dass insbesondere bei handlungsorientierten Motivationszielen eine besonders starke Disziplin vorherrschen muss, um die Kontrolle über die Vorhaben während eines längeren Zeitraums haben zu können.

Das wohl simpelste Modell in Bezug auf Motivation ist das so genannte Grundmodell der klassischen Motivationspsychologie nach Rheinberg (2002: 72). Dort wirken sowohl die individuellen Ziele einer Person bzw. eines Sportlers als auch die potenziellen Anreize einer bestimmten Situation auf die aktuelle Motivation ein, welche ausschlaggebend für das folgende Verhalten der Person ist. Eine Person ist also genau dann motiviert, wenn bedeutsame Motive 
in einer bestimmten Situation auftreten und aktiviert werden können (vgl. Dahlhaus 2007). Hier existiert eine deutliche Parallele zum Modell von Heckhausen (siehe Seite 48).

Ein weiteres überarbeitetes Modell einer Motivationstheorie ist die SelfDetermination Theory (SDT) nach Ryan \& Deci (u.a. 2007), welche sich zur Erläuterung von Motivation zum Sporttreiben und dem dahinterstehenden Verhalten eignet (vgl. Hagger \& Chatzisarantis 2008).

"Self-Determination Theory (SDT) is a theory of motivation. It is concerned with supporting our natural or intrinsic tendencies to behave in effective and healthy ways" (http://www.selfdeterminationtheory.org).

Die SDT unterscheidet drei verschiedene Bedürfnisse, nämlich zum einen das Bedürfnis nach Kompetenz, zum anderen nach Autonomie und schließlich nach Verbundenheit (vgl. Stoll, Pfeffer \& Alfermann 2010). „When these three needs are fulfilled, individuals feel that their motivation is ,self-determined': they are engaging in an activity out of choice and pleasure or because it reflects their values“" (Lapointe \& Perreault 2013: 137).

Neben der extrinsischen und intrinsischen Motivation beschreiben die gleichen Autoren Ryan \& Deci (2007) die so genannte Amotivation als einen „Zustand, bei dem entweder die Intention zum Handeln fehlt oder aber die Energie zu deren Umsetzung nicht aufgebracht werden kann“ (Stoll, Pfeffer \& Alfermann 2010: 225). Individuen nehmen daher im Gefühlsstatus der Amotivation nicht die möglichen Folgen von potenziellem Handeln wahr und bleiben deshalb passiv in ihrem Verhalten (vgl. Gillet, Vallerand \& Rosnet 2009). Darüber hinaus kann Amotivation zu einem ähnlichen Gefühl wie Inkompetenz führen (vgl. Guay, Vallerand \& Blanchard 2000). Auf den Zustand der Amotivation wird im weiteren Verlauf der Arbeit erneut eingegangen (siehe Seite 61). 
Der Grad der Selbstbestimmung (Self-Determination) fällt auf einem Kontinuum von intrinsischer Motivation über extrinsische bis hin zur Amotivation, bei welcher der Grad der Selbstbestimmung am geringsten nachzuweisen ist (vgl. Guay, Vallerand \& Blanchard 2000). Die erfolgreichsten Resultate werden daher bei intrinsisch motivierten Personen erwartet, die am wenigsten zufriedenstellenden Resultate folglich bei amotivierten Personen (vgl. Gillet et al. 2013). In ihrer neuesten Studie bestätigen Gillet et al. (2013) diese Theorie: „Indeed, SDT posits that autonomous [intrinsic] forms of motivation should faciliate performance as one is fully engaged on the task as engagement reflects a personal decision to partake in the activity" (477).

Umgekehrt gilt diese Aussage ebenfalls: Probanden, welche in einem direkten Vergleich bei einer Aufgabe gewinnen, sind höher intrinsisch motiviert als jene, welche verlieren. Auch wenn die intrinsische Motivation bei Verlierern geringer ist als bei Gewinnern, entwickelt sich in einigen Fällen ein besonderer Ehrgeiz, den Gegner das nächste Mal zu besiegen oder die Aufgabe besser auszuführen. Um bei Verlierern oder Misserfolgen auch die intrinsische Motivation wieder stärker zu fördern, hilft oftmals bereits eine positive Rückmeldung (vgl. Vansteenkiste \& Deci 2003).

Dies ist auch auf den nicht-wettkampforientierten Sport übertragbar: Kleine Rückschläge können durch positives Feedback oder ein einfaches Lob wieder wettgemacht werden, sodass sich auch kleine Erfolgserlebnisse wie ein Sieg für das eigene Selbstbewusstsein anfühlen und die intrinsische Motivation gesteigert wird.

Auch die extrinsische Motivation wird in der SDT noch in weitere SubKategorien unterteilt. Zum einen beschreibt die Steuerung von außen (external regulation) den Willen, eine Belohnung (sowohl finanzieller als auch psychischer Art) zu erhalten oder gegenteilig, Bestrafungen zu vermeiden. Des Weiteren existiert die Art von extrinsischer Motivation, welche zum aktiven Verhalten drängt, um Schuld und internen Druck zu vermeiden. Diese Art von Motivation ist oftmals durch bereits erlebte, vergleichbare Situationen geprägt. 
Eine dritte Komponente der extrinsischen Motivation beschreibt die freiwillige Teilnahme an einer Betätigung (identified regulation), obwohl diese auf Anhieb nicht notwendigerweise als attraktiv eingestuft wird (vgl. Guay, Vallerand \& Blanchard 2000). Zuletzt existiert die integrierte innere Steuerung, welche Verhaltensweisen des Individuums beschreibt, die im Wertesystem verankert sind, obwohl sie nicht zwingend mit Freude oder Spaß assoziiert werden (vgl. Gillet, Vallerand \& Rosnet 2009). Die SDT stellt einen effektiven Erklärungsversuch bezüglich dreier Schlüsselbereiche von Motivation und Handeln zur Verfügung, die im folgenden Zitat zusammengefasst werden:

\begin{abstract}
"First, it charts the antecedents and predictors of exercise behaviour, including factors in the environment (e.g. rewards, informational feedback, instruction style), and in the person (e.g. basic psychological need satisfaction) that affect motivational style or regulation in exercise contexts, exercise behaviour, and key psychological outcomes, such as intentions and perceived competence. Second, it provides some explanation of the mechanisms by which the antecedent constructs influence behaviour and other key outcomes; these include mediation and moderation effects. Finally, it provides useful guidelines on the exact constructs that psychologists and interventionists in the field of exercise promotion can target in order to change behaviour and motivate individuals to engage in more exercise" (Hagger \& Chatzisarantis 2008: 83).
\end{abstract}

\title{
4.3 Motive
}

Bei genauerer Betrachtung der Zielgruppe der Senioren wird schnell deutlich, dass es dieser nicht primär um das Abenteuer geht, sondern eher um gesundheitsfördernde Maßnahmen, auf die nun eingegangen wird. Im Folgenden werden Motive aus verschiedenen Studien konkret benannt und verglichen.

So ordnete Singer (1981) damals die Motive beispielsweise in fünf Kategorien ein, beginnend mit dem wichtigsten Motiv der Senioren zur sportlichen Betätigung:

- Gesundheit / Fitness

- Ausgleich / Abwechslung

- Freude / Spaß

- Geselligkeit / Kommunikation

- Leistung / Sportlicher Erfolg (85) 
Abele und Brehm (1990) beschreiben hingegen 10 Bereiche der Motivation zum Sporttreiben allgemein:

- Gesundheit und Fitness

- Wohlbefinden (Spaß/Wohlbefinden; Entspannung/Stressausgleich)

- Aussehen (sportliche Figur; Gewichtsreduktion)

- Leistung (Anstrengung/Belasten; Leistungsverbesserung; Vergleich/Wettkampf)

- Körpererfahrung

- Gemeinschaftserleben

- Kontakte (Bekanntschaften pflegen; neue Bekanntschaften)

- Spannung und Neues beim Sport erleben

- Ästhetik der sportlichen Aktivität

- Selbstpräsentation (Beckmann, Fröhlich \& Elbe 2009: 518)

Hier treten bereits neue Aspekte in den Vordergrund, welche die Entwicklung des Sports und seine Ziele beleuchten. Dennoch spielt der Leistungsaspekt bei Senioren sicher eine eher untergeordnete Rolle.

In einer jüngeren Studie kategorisiert Fuchs (1997) den Schwerpunkt der Antworten der Seniorensportler in drei Unterpunkte:

- Fitness / Gewichtskontrolle

- Aufrechterhaltung der Gesundheit / Sich-Besser-Fühlen

- Körperliches Wohlbefinden / Gesundheit (24)

Das Thema der Gewichtskontrolle scheint mehr und mehr an Bedeutung zu gewinnen, während das natürliche Sporttreiben rückläufig ist. In einem Zeitschriftenartikel behauptet Huber (2011), dass sich das natürliche Sporttreiben in Form von jagen oder Nahrung suchen innerhalb der letzten 100 Jahre drastisch reduziert habe und daher eine fehlende Motivation in Bezug auf das Sporttreiben möglicherweise sogar evolutionär bedingt sein könne. Daraus 
folgert er, dass der „evolutionär betrachtet urplötzliche Wechsel zu einem sitzenden Lebensstil [...] sich als fatal für unsere Gesundheit“ (109) erweise und es somit vermehrt zu auftretendem Übergewicht oder Adipositas komme.

Grundsätzlich ist es dennoch wichtig zu beachten, dass die Art der Ziele, die im Sport verfolgt werden, stark vom Alter der Zielgruppe abhängt. So sind Ziele, wie beispielsweise Sieg, Anerkennung und Perfektion, eher Ziele der jüngeren Altersgruppen, während sich der Alterssport deutlich an sozialen Einflüssen, Emotionen, Erwartungen und Selbstkontrolle orientiert (vgl. Fuchs 1997).

Soziale Einflüsse sind allerdings vom Alter unabhängig, wenn es um den Zugang zur sportlichen Aktivität geht. Bei Betrachtung der jüngeren Bevölkerung ist zunächst das Sportverhalten der Eltern von großer Bedeutung, da die Wahrscheinlichkeit, dass die Kinder auch Sport treiben bei sporttreibenden Eltern höher ist als bei nicht-sporttreibenden Eltern. Im weiteren Verlauf des Lebens gibt es unterschiedliche Arten der sozialen Unterstützung: Zunächst einmal existiert die informationelle Unterstützung, in der es hauptsächlich um sportmedizinisches Fachwissen seitens der Aktiven sowie sinnvolle Hintergrunderklärungen geht. Zweitens gibt es die instrumentelle Unterstützung, welche sich mit der Organisation von Sportevents und der Technik beziehungsweise dem Transport zu Sportstätten befasst. Als dritte Kategorie spielt die (verbale) Unterstützung die vermutlich wichtigste Rolle, die durch Lob und Kritik charakterisiert ist.

Daher wird das Sporttreiben aus Sicht der Unterstützungsforschung auch als „ein Mediator [bezeichnet], der den Zusammenhang zwischen Rückhalt und Gesundheit vermitteln kann“ (Fuchs 1997: 53).

Die wichtigste personelle Art der Unterstützung kommt meist von Seiten der Partner, Freunde, Kollegen oder Trainer. 
Am deutlichsten kommt die gesellige Komponente während des Sports in Gruppen oder Mannschaften zur Geltung. Hierbei sorgt bereits die Tatsache, dass andere Gruppenmitglieder den Sporttermin wahrnehmen, für eine positive Einstellung und Motivation dem Training gegenüber, damit die anderen Teilnehmer nicht im Stich gelassen werden. Des Weiteren kommt es innerhalb von Gruppen häufig zu einer bestimmten Dynamik, da die Teilnehmer im Normalfall die gleichen Ziele verfolgen. Das so genannte „Anschlussmotiv“ ist charakterisiert durch den Wunsch nach Anschluss in einer Gruppe. Es hat sowohl eine „aufsuchende (Hoffnung auf Anschluss) [als auch] eine meidende (Furcht vor Zurückweisung) Komponente“ (Beckmann, Fröhlich \& Elbe 2009: 532). Klappt die Integration in eine Gruppe schnell und effektiv, ist dieses Motiv positiv geprägt, gibt es allerdings Anschlussschwierigkeiten, kann dieses Motiv auch negativ geprägt und als Drucksituation interpretiert werden.

Ein anderer Aspekt, der auch als bedeutend für die Motivation beziehungsweise Teilnahme am Sport gilt, ist die Emotion, welche als Voraussetzung für das Sporttreiben charakterisiert wird (vgl. Gabler 2000).

„Emotionen sind als Prozesse zu verstehen, die verschiedene Reaktionskomponenten beinhalten. Dazu gehören die kognitive Komponente, die physiologische Komponente (Erregung), die subjektive Komponente (Gefühl), die motorische Komponente (Verhaltenstendenz) und die Ausdruckskomponente (Mimik, Haltung)“ (Dahlhaus 2007: 229).

Hier wird deutlich, dass Motivation und Emotion in engem Zusammenhang stehen. So charakterisiert das Motiv ,Spaß' beispielsweise die Emotionen der Freude an Bewegung, Glück bei Erfolgen oder sportlich angenehme Erschöpfung (vgl. Gabler 2002). Ein Verhalten wird daher häufig durch eine gewisse Stimmungslage ausgelöst, welche als Reaktionsmuster agiert. Konkrete Beispiele für Emotionen sind etwa „Ärger, Anspannung, Befriedigung, Entspannung, Enttäuschung, Freude, Furcht, Hoffnung, Langeweile, Missbefinden, Lust, Stolz, Stress, Unlust, Wohlbefinden und Zufriedenheit“ (Gabler 2002: 170). Lazarus $(1991,2000)$ fügt diesen noch folgende hinzu: „Schuld, Scham, Trauer, Abscheu, Angst, Erschrecken, Neid, Eifersucht, Glück, Erleichterung [und] Mitgefühl“ (Reicherts \& Horn 2009: 576). Die direkte Bewertung eines Geschehens drückt sich mit der jeweiligen Emotion aus. 
Einige dieser oben genannten Emotionen beziehen sich vornehmlich auf den Wettkampf- und Leistungssport und verlieren im gesundheitsorientierten Sport an Bedeutung.

Zudem verstärken Emotionen, wie Hoffnung oder Furcht, die allgemeine Erwartungshaltung. Emotionen sind jedoch nicht nur Voraussetzungen für sportliche Betätigungen, sondern ebenso Begleiterscheinungen oder Folgeerscheinungen (vgl. Gabler 2002).

Außerdem, und dies ist ein weiterer Aspekt, spielen die Erwartungen an ein Sportprogramm eine wichtige Rolle. Hierbei wird erneut je nach Situation unterschieden. Handelt es sich bei den Seniorensportlern um Neuanfänger, herrschen eher generalisierte Erwartungen vor; sind die Sportler schon lange aktiv, spricht man eher von situationsspezifischen Erwartungen, da die groben Abläufe bereits bekannt sind.

Bei einer Erwartung, also der "gedanklichen Antizipation zukünftiger Ereignisse oder Zustände" (Fuchs 1997: 76), sind immer auch die Konsequenzen mit zu berücksichtigen. Diese lassen sich nach Banduras (1986) in drei verschiedene Unterpunkte gliedern: die körperlichen, sozialen und selbst-evaluierten Konsequenzen. Die körperlichen Konsequenzen können sowohl positiv, wie zum Beispiel bei einem angenehmen Erschöpfungsgefühl, als auch negativ, wie zum Beispiel bei einer Verletzung, ausfallen. Auch bei den sozialen Konsequenzen lässt sich zwischen positivem Lob oder negativer Kritik anderer unterscheiden. Die selbst-evaluierten Konsequenzen beschreiben die allgemeine persönliche Gefühlslage nach der sportlichen Betätigung, welche oftmals ebenfalls abhängig von sozialen oder physischen Konsequenzen ist.

\section{4 ,Attentional Involvement' und ,Flow‘}

Die Stärke der Aufmerksamkeit (,attentional involvement') ist ein weiterer Faktor, welcher im Rahmen der Motivationsforschung während der 
Durchführung einer Aufgabe empirisch erfasst werden kann. Wenn die Aufmerksamkeit besonders stark auf der Durchführung der Aufgabe liegt, so ist der Proband oder die Probandin so stark involviert, dass bestenfalls keine Störgrößen oder Ablenkungen wahrgenommen werden. Der Spaßfaktor ist bei stark fokussierter Aufmerksamkeit am höchsten (vgl. Abuhamdeh \& Csikszentmihalyi 2012).

Zusätzlich lässt sich mit hoher Wahrscheinlichkeit sagen, dass das ,attentional involvement' eine Komponente von intrinsischer Motivation oder zielorientiertem Verhalten ist. Dies ist wiederum erneut lediglich durch quantitative Studien, wie z.B. jene von Abuhamdeh \& Csikszentmihalyi (2012), zu belegen. Hier wird unter anderem das ,attentional involvement' mithilfe eines computergestützten Fragebogens abgefragt, indem die Befragten auf einer Skala von 1 (kaum) bis 6 (sehr) angeben müssen, wie gut sie sich bei ihrer Aufgabe konzentrieren können und wie stark sie in die Aufgabe involviert sind (vgl. Abuhamdeh \& Csikszentmihalyi 2012). Es lässt sich feststellen, dass eine hohe Aufmerksamkeitsspanne grundsätzlich eine intensivere Übungsausführung ermöglicht. Nichtsdestotrotz kann eine Aufmerksamkeitslenkung auf andere Tätigkeiten (abseits von der Hauptaufgabe) ebenso einen hohen Spaßfaktor generieren.

Übertragen auf ein sportliches Umfeld ist diese Erkenntnis von Interesse, da manchmal eben gerade nicht das strikte Trainieren einer expliziten Übung Freude bereitet, sondern eher Abwandlungen, welche für Abwechslung und neue Anreize sorgen.

Dennoch kann eine starke Aufmerksamkeitslenkung auf eine Aufgabe in extremen Fällen zum so genannten „Flow-Erleben“ führen (vgl. Abuhamdeh \& Csikszentmihalyi 2012). Dieser Zustand ist von Csikszentmihalyi (1975) definiert als "das selbstreflexionsfreie, gänzliche Aufgehen in einer glatt laufenden Tätigkeit, bei der man trotz voller Kapazitätsauslastung das Gefühl hat, den Geschehensablauf noch gut unter Kontrolle zu haben" (Rheinberg 2006: 345). Dieser Zustand tritt in einigen Fällen auch bei nicht leistungsorientierten Aktivitäten auf und unterstützt das Gefühl, eine Tätigkeit 
ihrer selbst willen durchzuführen, um das Gefühl wahrnehmen zu können, die Bewegung an sich zu genießen (z.B. Tanzen) und nicht auf eine externe Belohnung hinzuarbeiten (vgl. Rheinberg, Vollmeyer \& Engeser 2003, Rheinberg 2006). Des Weiteren ist das ,Flow-Erleben' gekennzeichnet durch Freude und Glück, welche sich lediglich in ihrem Umfang unterscheiden: "Während Glück Zufriedenheit umfasst, kann Freude zu Ekstase werden“ (Reicherts \& Horn 2009: 601).

Folgende Komponenten des ,Flow-Erlebens' werden von Rheinberg, Vollmeyer \& Engeser (2003) zusammengefasst:

1. „Man fühlt sich optimal beansprucht und hat trotz hoher Anforderung das sichere Gefühl, das Geschehen noch unter Kontrolle zu haben (Balance zwischen Anforderung und Fähigkeit).

2. Handlungsanforderungen und Rückmeldungen werden als klar und interpretationsfrei erlebt, sodass man jederzeit und ohne nachzudenken weiß, was jetzt als richtig zu tun ist.

3. Der Handlungsablauf wird als glatt erlebt. Ein Schritt geht flüssig in den nächsten über, als liefe das Geschehen gleitend wie aus einer inneren Logik. (Aus dieser Komponente rührt wohl die Bezeichnung „Flow“.)

4. Man muss sich nicht willentlich konzentrieren, vielmehr kommt die Konzentration wie von selbst, ganz so wie die Atmung. Es kommt zur Ausblendung aller Kognitionen, die nicht unmittelbar auf die jetzige Ausführungsregulation gerichtet sind.

5. Das Zeiterleben ist stark beeinträchtigt; man vergisst die Zeit und weiß nicht, wie lange man schon dabei ist. Stunden vergehen wie Minuten.

6. Man erlebt sich selbst nicht mehr abgehoben von der Tätigkeit, man geht vielmehr gänzlich in der eigenen Aktivität auf (sog. „Verschmelzen“ von Selbst und Tätigkeit). Es kommt zum Verlust von Reflexivität und Selbstbewusstheit." (263)

So gut das ,Flow-Erleben' auch aus ggf. eigenen Erfahrungen nachzuvollziehen ist, umso schwieriger erweist sich ein Messen oder Darstellen dieses Gefühls in 
Zahlen, Graphiken oder Worten (vgl. Rheinberg 2002). So wurde zwar bisher versucht, dieses Phänomen mittels Angaben auf Skalen über aktuelle Gefühlszustände zu messen, jedoch führen alle Probanden verschiedene Aufgaben durch, sodass Anforderung und Herausforderung nicht klar voneinander getrennt werden. Auch die Flow-Kurzskala, auf der zehn Items auf einer 7-Punkte-Skala bewertet werden sollen, ist kritisch zu betrachten, da alle Probanden ihre Aufgaben kurzzeitig unterbrechen müssen, um die Skala auszufüllen. Allein die kurze Unterbrechung führt zu einem abrupten Ende eines möglichen Flow-Zustandes, welcher daher in der Skala gar nicht hundertprozentig wiedergegeben werden kann (vgl. Rheinberg, Vollmeyer \& Engeser 2003).

\subsection{Ziele sportlicher Betätigung im Alter}

Da die Ziele der sportlichen Betätigung im Alter in dieser Arbeit eine besonders wichtige Rolle spielen, sollen sie hier gesondert aufgeführt werden. Das übergeordnete Ziel des Seniorensports ist und bleibt die Erhaltung bzw. Förderung der Gesundheit und die damit verbundenen Verbesserungen des persönlichen Wohlbefindens und der erhöhten Lebensqualität. Um diese Ziele erreichen zu können, ist ein starker Wille notwendig sowie eine positive Einstellung dem Altern gegenüber, damit degenerativen Veränderungen im Alter entgegengewirkt werden kann (vgl. Tomasch 1997).

Gesundheitlich gesehen wird versucht, mithilfe der sportlichen Betätigung Risikofaktoren zu vermeiden, also präventiv zu agieren. Der Begriff der Prävention bezieht sich entweder primär auf die „Vorbeugung von krankhaften Zuständen, die in der Altersphase aufgrund statistischer Erfahrungen zu erwarten sind [...]" (Müller, Rösch \& Wischmann 1979: 129) oder sekundär auf die „Vorbeugung gegenüber Komplikationen oder [...] [Vorbeugung der] Weiterentwicklung von Krankheiten nach deren Erstmanifestation" (Müller, Rösch \& Wischmann 1979: 129). 
Das körperliche Wohlbefinden an sich umfasst jedoch mehr als lediglich die Abwesenheit von Krankheiten. Es beinhaltet unter anderem die „Zufriedenheit mit dem momentanen Körperzustand“, das "Gefühl von Ruhe und Muße“, das „Empfinden von Vitalität und Lebensfreude“ und „Konzentration und Reaktionsbereitschaft“" (Dahlhaus 2007: 57).

Doch nicht nur der Gesundheitsaspekt steht auf der Liste der Ziele im Alterssport. Hinzu kommen weitere Faktoren, wie beispielsweise der Aspekt der Geselligkeit, also die soziale Komponente (vgl. Werle, Woll \& Tittlbach 2006). Gerade mit dem Eintritt in das Rentenalter können potenzielle soziale Kontakte, z.B. zu ehemaligen Arbeitskollegen, verloren gehen und somit kann der Sport alternativ neue Kontaktmöglichkeiten bieten (vgl. Kolb 1999). Hier leistet der Sportverein eine wichtige Aufgabe, indem er durch das Gruppenerleben und die familiäre Atmosphäre dazu beiträgt, soziale Isolation zu vermeiden. Häufig hilft auch das übliche Duzen innerhalb der Gruppe, schneller Anschluss zu finden. Obwohl diese Thematik als immens wichtig zu bewerten ist, gibt es bisher kaum Studien zu den positiven Effekten des Sports auf das soziale Wohlbefinden (vgl. Fuchs 2003).

Das erfolgreiche Altern, welches als grundsätzliches Ziel der älteren Generation anzunehmen ist, beschreibt das Gelingen des Übergangs von beruflicher Aktivität bis hin zum Rentenalter. Sport spielt hierbei eine entscheidende Rolle und verhindert oftmals das Auftreten von altersbedingten Depressionen, welche durch den Wegfall der beruflichen Tätigkeit in einigen Fällen auftreten können (vgl. Eichberg \& Mechling 2009).

\subsection{Mögliche Barrieren}

Mögliche Barrieren in Bezug zur aktiven sportlichen Betätigung sind in mehreren Bereichen zu finden. Zum einen existieren psychologische Barrieren, 
wie etwa ein Mangel an Selbstmotivation oder ein selbstbestimmter Zeitmangel, welcher gerade im Rentenalter oftmals als Ausrede oder Entschuldigung benutzt wird. Diese Barriere ist auch für jene relevant, die nicht regelmäßig Sport treiben, das heißt, dass diese zwar hin und wieder sportlich aktiv sind, die Kontinuität und der dauerhafte Wille jedoch fehlen und somit werden sie zu Gelegenheitssportlern (vgl. Fuchs 2003). Weiterhin gibt es psychische Barrieren, also der Glaube, dass es dem eigenen Körper gut gehe und Sport nicht vonnöten sei.

Zum anderen existieren sozioökologische Faktoren, wie zum Beispiel eine fehlende soziale Unterstützung, also ein sportlich inaktiver Freundeskreis oder ein fehlender Zugang zu Sportstätten, d.h. es gibt keine Sportstätten in Wohnortnähe. Oftmals werden aber auch die Gründe in der Ausstattung und in anderen Gegebenheiten der Sportstätten vor Ort bemängelt, beim Schwimmbad zum Beispiel „zu kaltes Wasser, fehlende Sicherheitsgriffe und Sicherheitsvorkehrungen beim Einstieg, steile Treppe [etc.]" (Müller, Rösch \& Wischmann 1979: 103).

Vielfach spielt sogar das Wetter eine entscheidende Rolle, ob ältere Leute sich sportlich betätigen oder nicht (vgl. Werle, Woll \& Tittlbach 2006). Hinzu kommen weitere, die Persönlichkeit des Individuums betreffende Argumente zum Nichtsporttreiben, wie beispielsweise das Gefühl, zu alt für Sport zu sein oder sich schonen zu müssen aus Angst vor Verletzungen oder weiteren degenerativen Veränderungen (vgl. Dahlhaus 2007).

Als weitere Gründe geben Nichtaktive ein simples Desinteresse an Sport oder andere Hobbys an, nur in seltenen Fällen spielt der finanzielle Aspekt eine Rolle (vgl. Denk, Pache \& Schaller 2003).

Neben der Studie von Ryan \& Deci (2007) (vgl. Seite 50) herrscht auch in einigen englischen Studien der Begriff der „Amotivation“ (vgl. z.B. Vlachopoulos \& Gigoudi 2008: 316 f. oder De Pero et al. 2009: 61 f.) vor, welcher das 
Gegenteil des Prozesses der Motivation umschreibt. Der Begriff der Amotivation ist im englischsprachigen Raum folgendermaßen definiert: „Amotivation refers to a lack of intentionality and thus the relative absence of motivation" (Vlachopoulos \& Gigoudi 2008: 317). Es mangelt den Seniorinnen und Senioren demnach, bezogen auf die Sportthematik, an dem Wunsch bzw. der Absicht, Sport zu treiben.

Eine Studie von Cohen-Mansfield, Marx und Guralnik (2003), welche im Journal of Aging and Physical Activity publiziert wurde, handelt von genau dieser Thematik. Dort werden 324 Seniorinnen und Senioren im Alter von 74 bis 85 Jahren mithilfe eines Fragebogens befragt, ob sie Sport treiben und im Falle einer negativen Antwort wird nach Gründen oder Barrieren geforscht, welche für das Nichtsporttreiben sorgen. Innerhalb des Fragebogens wird einerseits nach der allgemeinen Gesundheit („general health“: 246) auf einer 5-stufigen Skala gefragt. Andererseits wird das Gebiet des Schmerzes („pain“: 246) auf einer Skala von 1 bis 10 untersucht. Außerdem werden durch offene Fragen mögliche Barrieren sowie Motive zum Sporttreiben erfasst.

Hierbei sind die am häufigsten genannten Barrieren Gesundheitsprobleme bzw. Schmerzen. Zudem werden Gründe wie beispielsweise fehlender Anreiz, fehlende Zeit, andauernde Müdigkeit oder schlechtes Wetter angegeben.

Bei den Motiven zum Sporttreiben werden überwiegend das gesteigerte persönliche Wohlbefinden und der gesundheitliche Aspekt genannt (,feeling better / improved health": 249).

Somit herrscht eine enge Korrelation zwischen Barrieren und Motiven, da es sich in den meisten Fällen um gegensätzliche Aspekte handelt. So ist der gesundheitliche Faktor einerseits ein Barrieregrund, andererseits auch ein motivierender Aspekt. 
Fest steht, dass in allen Fällen probiert werden sollte, eine Überwindung der Barrieren zu ermöglichen. Inwiefern das als realistisch einzustufen und realisierbar ist, wird im praktischen Teil dieser Arbeit analysiert (siehe S. 80 ff.).

\section{Demographie Deutschlands}

\subsection{Bevölkerungsentwicklung in Deutschland}

Die demographische Entwicklung ist eine weltweite Angelegenheit, welche hohe Aufmerksamkeit verlangt und welcher laut Kirkwood (2007) sogar mehr Bedeutung beigemessen werden sollte „als dem Klimawandel, der Globalisierung und dem Terrorismus [...]" (Rott 2008: 9). Der demographische Wandel wird in der Literatur als „Veränderungen der Bevölkerungsentwicklung, der Alters- und Geschlechtsstruktur, der ethnischen Zusammensetzung und der regionalen Verteilung der Bevölkerung sowie der Lebensformen" beschrieben und ist bestimmt durch „die Entwicklung der Anzahl der Geburten, der Sterbefälle und der Wanderungsbilanz" (Bundesministerium des Innern 2011: $11)$.

„Der Umgang mit den Auswirkungen der Alterung wird eine der wichtigsten politischen und gesellschaftlichen Herausforderungen der nächsten Jahrzehnte sein" (http://www.destatis.de). Diese Worte von Roderich Egeler, Präsident des Statistischen Bundesamtes, vom 18.11.2009 führten unter anderen in die Thematik zur 12. koordinierten Bevölkerungsvorausberechnung ein. Bei einer Bevölkerungsvorausberechnung handelt es sich um eine hypothetische Berechnung der Population unter Berücksichtigung unterschiedlicher Aspekte bzw. Annahmen. 
Grundsätzlich muss festgehalten werden, dass eine weiterhin gleich bleibend niedrige Geburtenrate über einen Zeitraum mehrerer Jahre in Kombination mit einer ansteigenden Lebenserwartung zu einer Alterung führt. Trotz der niedrigen Sterberate kommt es aufgrund der noch niedrigeren Geburtenrate zu einem Rückgang der Gesamtbevölkerung in Deutschland. Die Alterung beschreibt das „Phänomen [...], daß in einer Bevölkerung der Anteil an Menschen oberhalb eines bestimmten Alters, meist 60 oder 65 Jahre, stärker wächst, als der Anteil unterhalb dieser Grenze" (Kolb 1999: 23). Das folgende Balkendiagramm zeigt genau diese Entwicklung im Jahr 2008 und zum Vergleich im Jahr 2030:

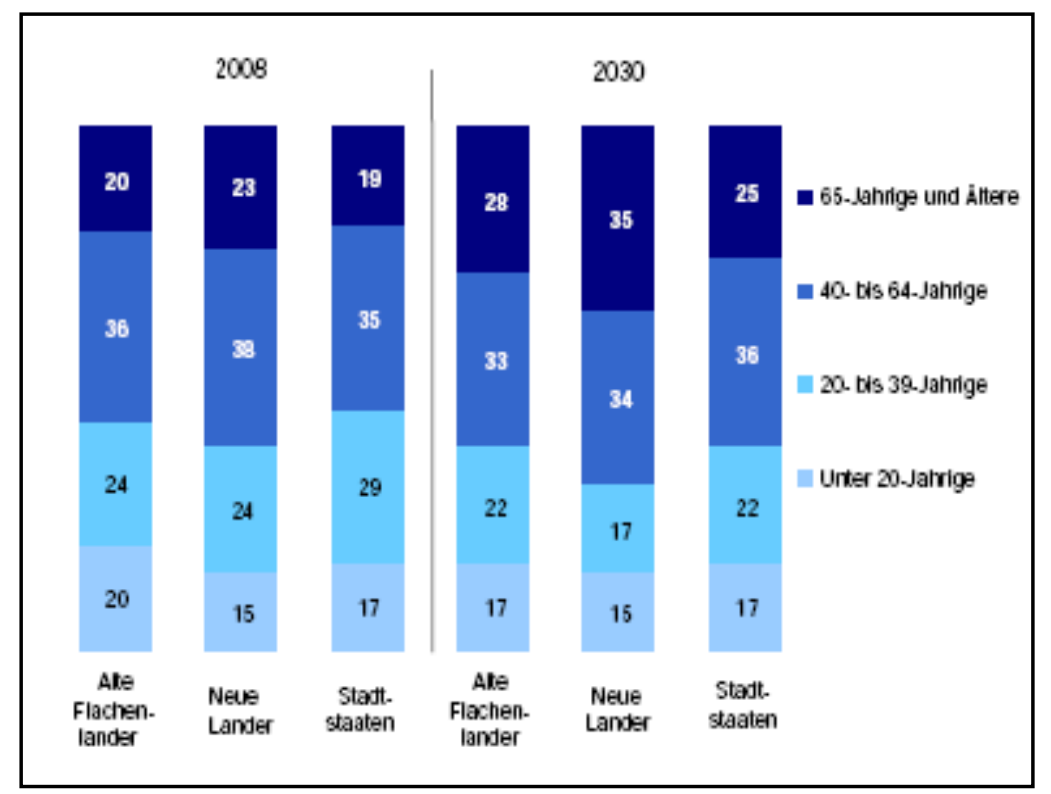

Abbildung 3: Bevölkerung nach Altersgruppen 2008 und 2030 (Variante der Vorausberechnung mit der mittleren Bevölkerung von drei möglichen Varianten) in \% der Gesamtbevölkerung (Statistisches Bundesamt 2011: 25).

Die Gründe für die Langlebigkeit der Bevölkerung liegen in einem „Zusammenspiel von steigendem Wohlstand, Bildung, gesunder Ernährung, humanen Arbeitsbedingungen mit geringerem körperlichem Verschleiß, verbesserter Hygiene, sozialer Fürsorge und medizinischer Vorsorge" (Rott 2008: 11) inklusive körperlicher Betätigung und präventiver sportlicher Aktivität. 
In seinem Statement auf der Pressekonferenz in Berlin erläuterte Präsident Roderich Egeler die Annahmen, die als Voraussetzung für die 12. Bevölkerungsvorausberechnung gesetzt wurden und eine Berechnung bis zum Jahr 2060 ermöglichen. Die Annahmen zur Geburtenhäufigkeit beziehen sich auf durchschnittlich 1,4 Kinder pro Frau, entsprechend dem aktuellen Durchschnitt. Die untere Grenze umfasst eine Durchschnittszahl von 1,2 Kindern pro Frau bis 2060 und die obere Grenze eine Zahl von 1,6 Kindern pro Frau bis 2025. Die Annahmen zur Lebenserwartung, der zweite Aspekt, lauten einmal für das Jahr 2060 für neugeborene Jungen 85,0 Jahre und für neugeborene Mädchen 89,2 Jahre. Als obere Grenze und zweite Annahme wird eine Lebenserwartung für das Jahr 2060 von 87,7 Jahre für neugeborene Jungen und 91,2 Jahre für neugeborene Mädchen vorausgesehen.

Zum besseren Vergleich: Die Werte zur Lebenserwartung betrugen im Jahr 2008 77,2 Jahre für neugeborene Jungen und 82,4 Jahre für neugeborene Mädchen. Generell gilt als sichere Aussage, dass die Lebenserwartung der Männer proportional stärker als die der Frauen ansteigen wird. Gründe hierfür sind beispielsweise die sich angleichenden beruflichen Tätigkeiten aufgrund der Emanzipation der Frau. Die folgende Graphik zeigt die noch bleibende Lebenserwartung ab einem Alter von 60 Jahren für Männer und Frauen bis zum Jahre 2060. Hier wird deutlich, dass die Lebenserwartung der Männer voraussichtlich auch im Jahr 2060 noch nicht an die der Frau heranreicht. Dieser Prozess wird demnach als längerfristig und sich langsam entwickelnd eingestuft. 


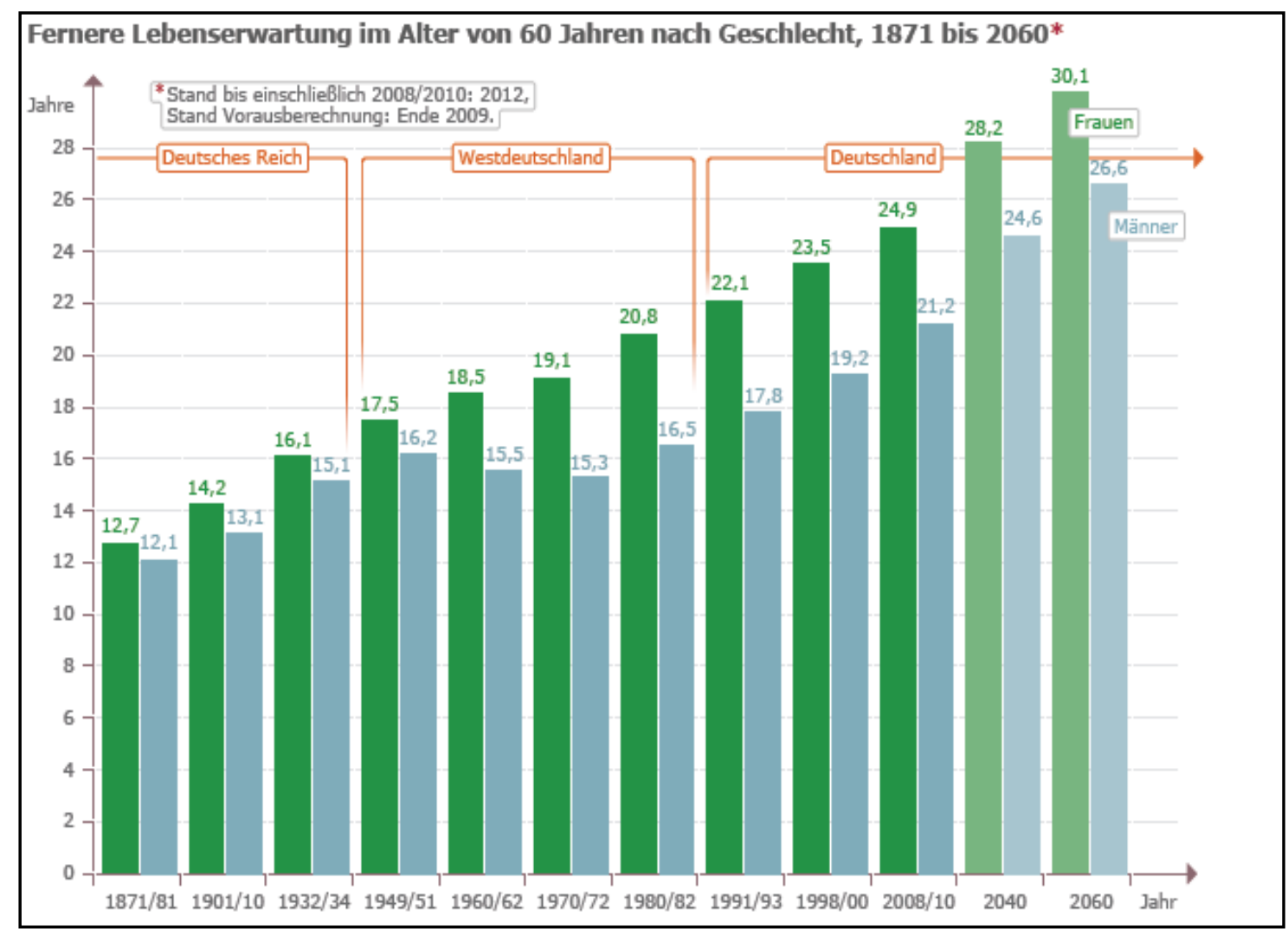

Abbildung 4: Lebenserwartung ab 60 Jahre für Männer und Frauen, 1871 bis 2060 (Statistisches Bundesamt 2011)

Als dritte und letzte Annahmekategorie wird das Wanderungssaldo genannt, also die Zuzüge in der Bundesrepublik Deutschland und die Abwanderungen aus dem Land. Die erste Annahme beschreibt einen Anstieg der Zahl auf 100.000 Menschen bis Ende 2014 mit anschließender Konstanz, während die zweite Annahme einen Anstieg auf 200.000 Menschen bis 2020 prognostiziert mit anschließender Konstanz. Selbstverständlich handelt es sich bei diesen Zahlen um Durchschnittswerte, da exakte Zahlen nur sehr schwer vorausgesagt werden können. 


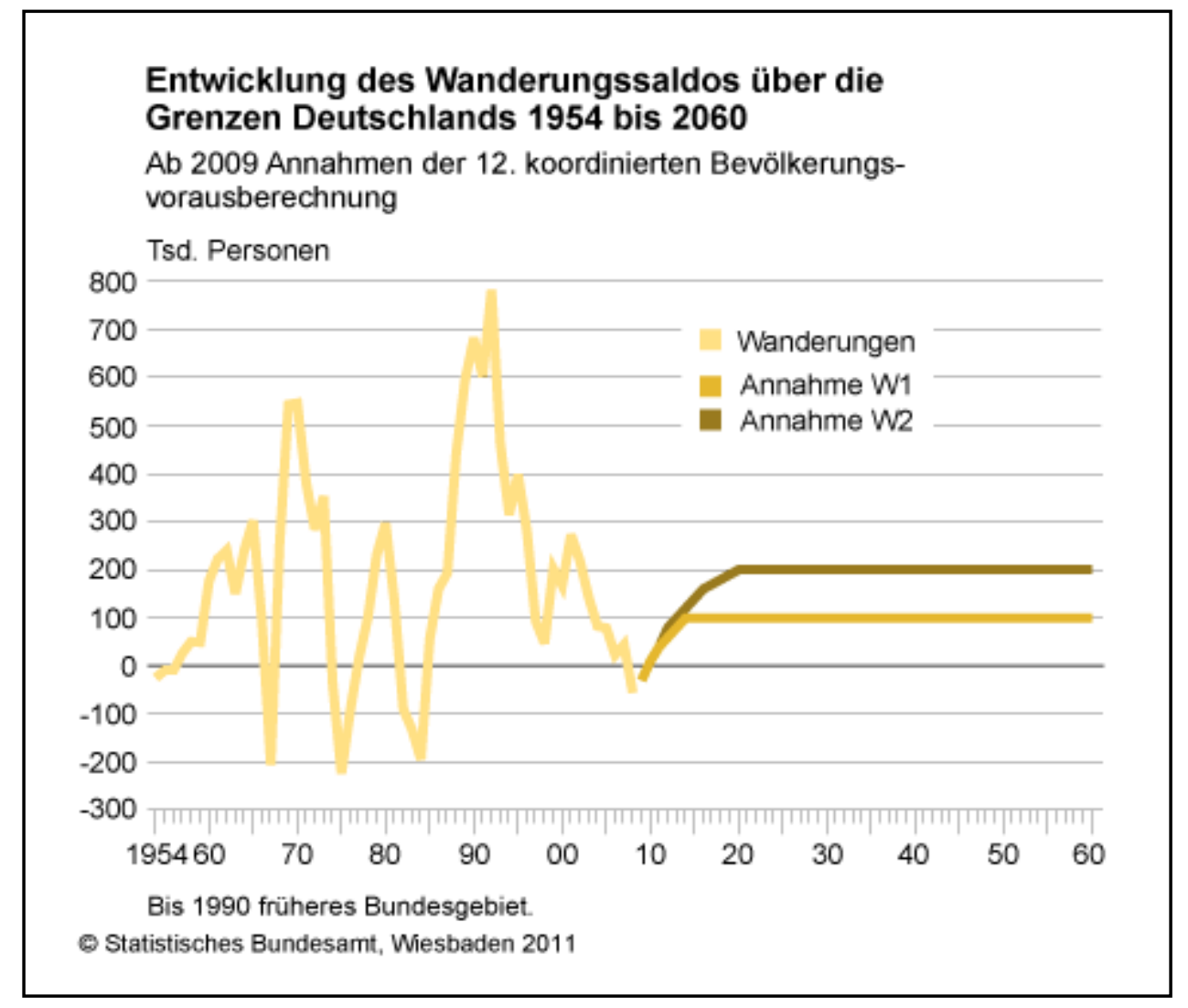

Abbildung 5: Wanderungssaldo 1954 bis 2060 (Statistisches Bundesamt 2011)

Die Bevölkerung ist in der Bundesrepublik über die letzten Jahre deutlich heterogener geworden: Im Moment verzeichnet die Bundesrepublik einen Anteil an Menschen mit Migrationshintergrund von 15,7 Millionen (vgl. Demografiebericht des Bundesministeriums des Innern 2011). Die Zuwanderung in den 50er und 60er Jahren ist hauptsächlich durch Arbeitsmigranten gekennzeichnet. In den 70er und 80er Jahren folgen die Familien jener, die zuvor eingewandert sind. Erst seit den 90er Jahren ändert sich die Hauptzuwanderungsstruktur, indem viele Asylsuchende, Flüchtlinge oder Saisonarbeiter zuwandern. Die meisten Zuwanderer ab den 90er Jahren stammen aus Europa, nämlich aus Polen, Rumänien und Bulgarien (vgl. Bundesministerium des Innern 2011). Als Migranten werden laut der Definition der Vereinten Nationen Personen betitelt, „die nicht in dem Land leben, in dem sie geboren wurden oder dessen Staatsbürgerschaft sie besitzen“ (Bundesministerium des Innern 2011: 76). 
Aus all diesen genannten Annahmen ergeben sich insgesamt zwölf Varianten zur Berechnung der Bevölkerungsentwicklung in Deutschland. Werden davon die Mittelwerte genommen, kommt es zu folgenden Ergebnissen: Während die Gesamtpopulation in Deutschland im Jahr 2008 etwa 82 Millionen Menschen betrug, wird sich diese Zahl im Jahr 2060 voraussichtlich auf etwa 65-70 Millionen reduzieren. Der Grund, warum die Bevölkerung schrumpft, liegt in der Tatsache, dass die Anzahl der Gestorbenen die Zahl der Neugeburten deutlich übersteigt, also die Sterberate höher ist als die Geburtenrate.

Was die Alterung innerhalb der Gesellschaft angeht, werden ebenfalls erhebliche Änderungen erwartet: Im Jahr 2060 wird prognostiziert, dass rund 60 Prozent der Bevölkerung 65 Jahre und älter sein wird. Des Weiteren wird für das Jahr 2060 ein Anstieg von 5 Prozent (Jahr 2008) auf 14 Prozent 80-jähriger und noch älterer Menschen vorausgesagt.

Zudem werden für das Jahr 2060 lediglich 36 Millionen Menschen im Berufsleben erwartet, was 27 Prozent weniger wären als heutzutage (vgl. http://www.destatis.de).

Das Phänomen der Bevölkerungsentwicklung in Deutschland wird auch als "dreifaches Altern“ (Tews 2001: 29) bezeichnet. Zunächst wird es durch die Zunahme des absoluten Alters älterer Menschen gekennzeichnet. Außerdem wird die Entwicklung des relativen Anteils an Seniorinnen und Senioren im Vergleich zum Anteil der jungen Generation wichtiger: „Wenn der Anteil der nachrückenden jüngeren Altersgruppe sinkt [(Rückgang der Geburtenrate)], so nimmt der relative Anteil der Älteren zu, auch wenn deren absolute Zahl gleich bleibt“" (Kolb 1999: 29).

Als letzten Punkt geht es um einen noch deutlicheren Zuwachs der Hochaltrigen, also der Menschen, die mindestens das achtzigste Lebensjahr erreicht haben. So ist zu erwarten, dass laut Sterbetafel 2004-2006 68 Prozent der weiblichen Senioren und 49 Prozent der männlichen Senioren davon ausgehen können, 80 Jahre alt zu werden (vgl. Rott 2008). 
Folgendes Schaubild zur Entwicklung der Altersstruktur verdeutlicht erneut den Zuwachs von älteren Menschen an der Gesamtpopulation. Hier wird die Bevölkerung aus dem Jahr 2008 mit der voraussichtlichen Bevölkerung von 2060 verglichen. Es zeigt sich auf den ersten Blick, dass das Durchschnittsalter deutlich steigen wird und auch die Anzahl der Hochaltrigen insgesamt ansteigen wird. Während im Jahr 2008 kaum Frauen, geschweige denn ein Mann das Alter von 100 Jahren erreicht, sieht dies im Jahr 2060 voraussichtlich deutlich anders aus:

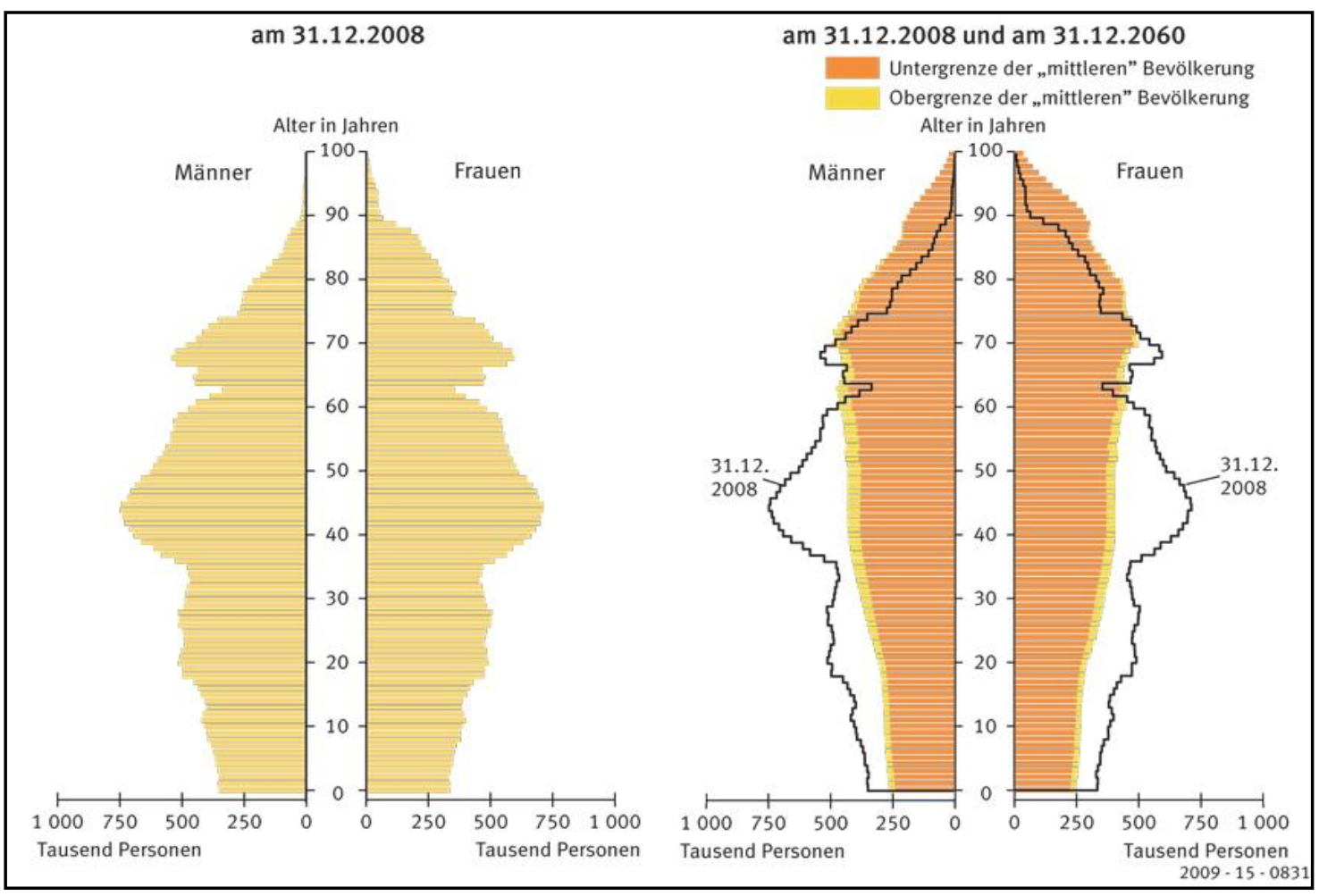

Abbildung 6: Altersaufbau der Bevölkerung in Deutschland (Statistisches Bundesamt 2011).

Aufgrund dieser Entwicklung rückt der Fokus auf die Gesundheit der Menschen, gerade jener im hohen Alter, sodass die Forderung nun nicht mehr ausschließlich „Add Years to Life!', sondern vielmehr „Add Life to Years“ [lautet], oder wie es noch deutlicher Junge (1978) formuliert: ,[...], dass es weniger darum ginge, länger zu leben, sondern gesünder zu sterben“" (Stemper 2001: 4). Diesbezüglich existieren zwei Thesen, welche das Thema Langlebigkeit diskutieren. Zum einen herrscht die Befürchtung, dass die 
Langlebigkeit den Hochaltrigen keine gewonnenen Lebensjahre beschert, sondern lediglich den Tod hinauszögert, ohne den Menschen ein schönes Altern zu ermöglichen. Zum anderen wird jedoch postuliert, dass Hochaltrige heutzutage gesünder altern und Krankheiten verhältnismäßig erst später im Lebenslauf auftreten (vgl. Perrig-Chiello 2008). Das folgende Diagramm zeigt die Pflegebedürftigkeit nach Alter und Geschlecht über die Lebensjahre aus dem Jahr 2005. Hier wird deutlich, dass die Pflegebedürftigkeit in sehr hohem Alter nicht zu ignorieren ist:

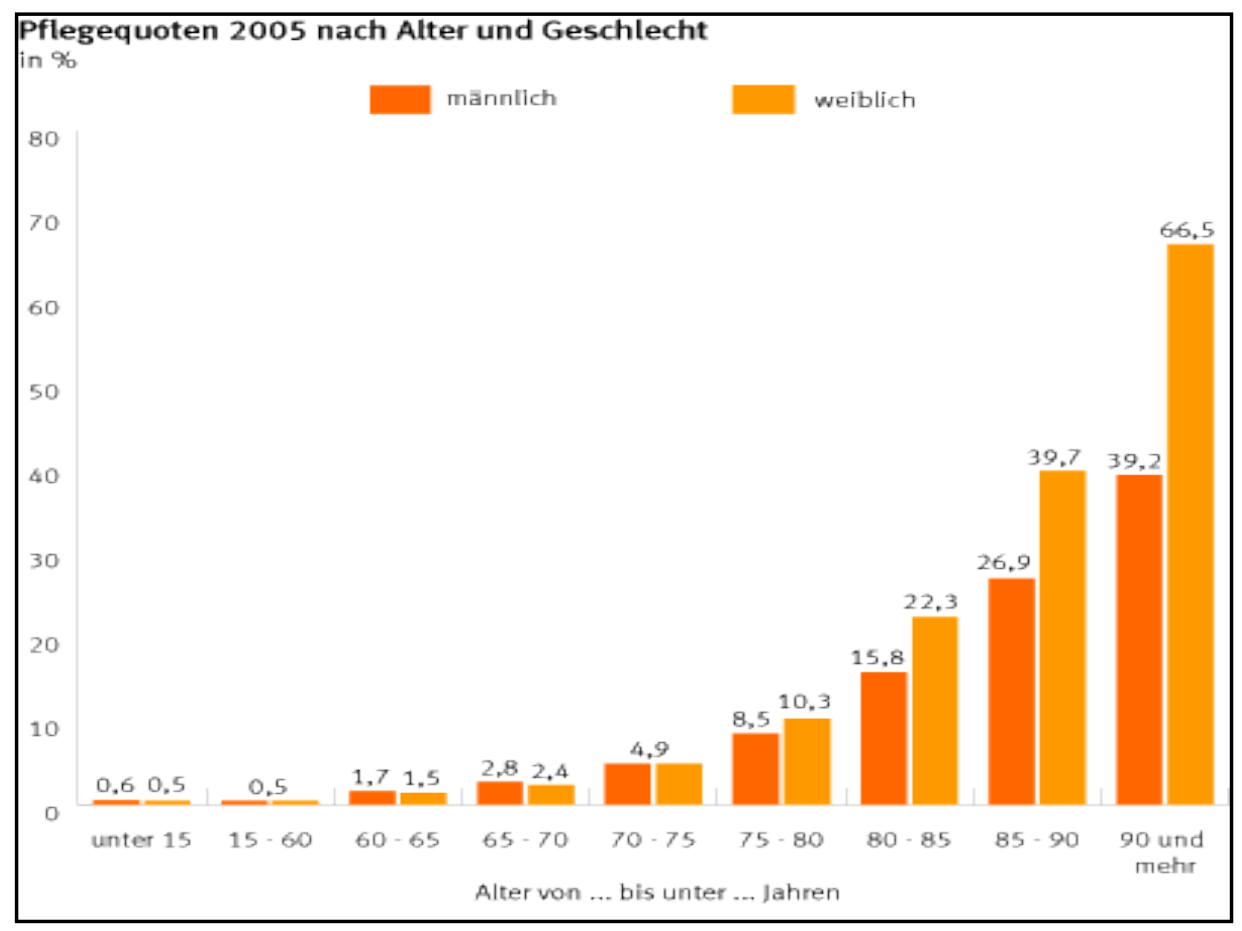

Abbildung 7: Pflegequoten 2005 (Statistisches Bundesamt 2011)

Werden entsprechende vorbeugende Maßnahmen unternommen, so rückt die zweite These in den Vordergrund und beschreibt ein wichtiges Ziel im Bereich der Gerontologie. Hier existieren Studien, welche belegen, dass die „heutigen Alten [...] durchschnittlich vitaler und aktiver [sind] als die der Väter- und Großvätergeneration [...]" (Schlicht \& Schott 2013: 9). Zudem ist heutzutage der durchschnittliche Gesundheitszustand eines 80-Jährigen zu vergleichen mit dem Gesundheitszustand eines 70-Jährigen von vor etwa 20 Jahren (vgl. Staudinger 2012). 
Tews (2001) beschreibt darüber hinaus weitere Aspekte des Strukturwandels im Alter generell. Dort wird erstens die Verjüngung des Alters genannt, welche sich auf die Entwicklung der Schönheitschirurgie und der Kosmetikindustrie bezieht, da ältere Menschen versuchen sich optisch mit allen möglichen Mitteln zu verjüngen. Zweitens geht es um die so genannte „Entberuflichung des Alters" (Tews 2001: 36), die das Herabsetzen des Renteneintrittsalters durch Frührente oder Altersteilzeit beschreibt und daher in Kombination mit der erhöhten Lebenserwartung eine längere Zeit ohne berufliche Aktivität voraussagt (vgl. Kolb 1999).

Der zweite Punkt gilt nur noch sehr eingeschränkt, denn es gibt seit 2007 ein neues Gesetz zum Renteneintrittsalter. Aufgrund der sinkenden Geburtenrate in Kombination mit der höheren Lebenserwartung wurde ein Gesetz zur Anhebung des Rentenalters auf langfristig 67 Jahre verabschiedet. Da die Unterstützung der arbeitenden Bevölkerung für die Rentenbezieher laut des Generationenvertrages langfristig nicht mehr gewährleistet werden kann, wird das Renteneintrittsalter ab dem Jahr 2012 schrittweise von 65 auf 67 Jahre erhöht, beginnend mit dem Geburtsjahrgang 1947. Ab 2012 soll in monatlichen Schritten erhöht werden, ab 2024 in zweimonatlichen Schritten für den Jahrgang 1959 und jünger. Daraus ergibt sich, dass die Geburtsjahrgänge ab 1964 komplette zwei Jahre länger arbeiten müssen als heute. Ausnahmen sind hierbei in Einzelfällen möglich ( $\mathrm{vgl}$. Bundesministerium des Innern 2012). Eine dieser Ausnahmen ist momentan aktuell (Stand Mai 2014), da durch die SPD ein Gesetz in Kraft getreten ist, das Menschen bestimmter Jahrgänge, die bereits 45 Jahre gearbeitet haben, erlaubt, in Rente zu gehen. Diese Rente mit 63 ist zwar nun beschlossen, wird aber immer noch diskutiert (vgl. Süddeutsche Zeitung 19.05.2014). So gibt es auch hier eine stufenweise Erhöhung des Alters: „Für ab 1953 Geborene steigt die Altersgrenze mit jedem Jahrgang um zwei Monate. Für den Jahrgang 1964 liegt sie somit bei 65 Jahren“ (Süddeutsche Zeitung 19.05.2014). 
Drittens wird die Feminisierung des Alters genannt, welche den momentan noch deutlich größeren Anteil der weiblichen Bevölkerung im hohen Alter beschreibt. Dieses Phänomen geht allerdings, wenn auch sehr langsam, nach und nach zurück (vgl. Schlicht \& Schott 2013). Viertens wird der Punkt der Singularisierung beschrieben, welcher eng mit der Feminisierung verwandt ist, da er die Alleinstehenden im hohen Alter beschreibt, die größtenteils Witwen sind. Auch hier ist ein Wandel über die Jahre zu sehen, sodass dieser Punkt langfristig an Aktualität verlieren wird. Als fünften und letzten Punkt nennt Tews (2001) die Hochaltrigkeit an sich, die in einigen Fällen geprägt wird von Krankheiten, sozialer Isolation und Pflegebedürftigkeit: „Bei den 75-Jährigen sind über $25 \%$ multimorbide; das Gros der Krebsneuerkrankungen entfällt auf Personen jenseits des 65. Lebensjahres und bei den über 80-Jährigen leiden etwa $40 \%$ an einer Form der Demenz (Alzheimer oder kardiovaskuläre Demenz)“ (Schlicht \& Schott 2013: 9).

Hier rückt die evolutionäre Entwicklung in den Vordergrund, da der Mensch ursprünglich lediglich eine wichtige Aufgabe hatte, nämlich für Nachkommen zu sorgen und diese großzuziehen. Mit Beginn der Hochaltrigkeit lassen sich also die sich entwickelnden Funktionsbeeinträchtigungen und gesundheitlichen Einschränkungen als logische Konsequenz einstufen, da der menschliche Körper für dieses hohe Alter ohne präventive Maßnahmen evolutionär schlichtweg nicht ausgelegt ist (vgl. Rott 2008). Aus diesem Grund verabschiedete die Bundesregierung im März 2013 einen Gesetzentwurf, welcher einen spezifischen Fokus auf die Primärprävention legt, und deshalb sollen die Krankenkassen diesen Bereich von nun an verstärkt fördern. Auch der betrieblichen Gesundheitsförderung wird ein zukunftsweisender Schwerpunkt zugesprochen (vgl. Bundesministerium des Innern 2013). Durch diese Maßnahmen wird erhofft, eine möglichst lange Selbständigkeit bis ins hohe Alter gewährleisten zu können. 


\subsection{Voraussichtliche Konsequenzen für die Entwicklung des Seniorensports}

Die aktuelle Bevölkerungsentwicklung wird als der entscheidende Grund für die positive Entwicklung des Alterssports angesehen (vgl. Meusel et al. 1980). Durch die einfache Tatsache, dass der Teil der älteren Bevölkerung innerhalb der nächsten Jahrzehnte kontinuierlich wachsen wird, ist das Thema des Alterssports unumgänglich geworden in der heutigen Zeit. Mit dem Eintritt in das Seniorenalter ergeben sich einige Einschränkungen, die zuvor bereits in Kapitel 3.4 beschrieben wurden. Gerade bei den Hochaltrigen herrscht oftmals eine Multimorbidität vor, also ein vermehrtes und gleichzeitiges Auftreten von altersspezifischen Krankheiten bei einem Individuum (vgl. Werle, Woll \& Tittlbach 2006).

Umso wichtiger wird die Bedeutung des Alterssports, welcher gerade diesen Krankheiten präventiv entgegenwirken soll. Ziele sind hierbei nicht nur die möglichst lange Selbständigkeit der Senioren, sondern auch eine Senkung von Betreuungs-, Pflege- und Gesundheitskosten im Falle einer guten Prävention und positiver Auswirkungen des Alterssports (vgl. Bundesministerium des Innern 2013). Außerdem herrscht das Ziel der Sensibilisierung der Seniorinnen und Senioren für eine größere Selbstverantwortung bezüglich der eigenen Verfassung vor, gerade weil die Älteren einen hohen Anteil der Gesellschaft ausmachen und diese repräsentieren (vgl. Denk, Pache \& Schaller 2003).

Bereits kurzweilige moderate Aktivitäten, welche jedoch regelmäßig ausgeführt werden sollten, können in vielen Fällen für eine längere Selbständigkeit im Alter sorgen - ein Ziel, welches nicht nur für die Gesellschaft, sondern auch für jedes Individuum von hohem Interesse sein sollte (vgl. Rott 2008). So hat sich der Deutsche Olympische Sportbund beispielsweise als Ziel vorgenommen, Sportangebote spezifisch auf ältere Menschen abzustimmen und zu entwickeln (vgl. Bundesministerium des Innern 2012). 


\subsection{Altersstatistiken des Deutschen Olympischen Sportbundes (DOSB)}

Um die Wichtigkeit des Sporttreibens - insbesondere im Seniorenalter - noch konkreter anhand von Zahlen zu belegen, werden im Folgenden die Mitgliederzahlen der Landessportbünde im Deutschen Olympischen Sportbund (DOSB) aufgezeigt und analysiert.

Die aktuelle Gesamtmitgliederzahl in allen Landessportbünden und Landessportverbänden des DOSB beträgt 23.691.030. Es gibt deutschlandweit 90.802 Sportvereine ( $v g l$. www.dosb.de). Vergleicht man die Mitgliederzahlen der Landessportbünde aus dem Jahr 2004 mit den aktuellen Zahlen aus dem Jahr 2014, fällt auf, dass die Mitgliederzahlen in der Bevölkerungsgruppe von 60 Jahren und älter deutlich zugenommen haben innerhalb der letzten zehn Jahre. Während im Jahr 2004 insgesamt 2.021.634 Männer ab 60 Jahren und 1.200.213 Frauen ab 60 Jahren unter den registrierten Mitgliedern der Landessportbünde des DOSB waren, sind es in der jüngsten Bestandserhebung aus dem Jahr 2014 bereits insgesamt 2.497.852 Männer ab 60 Jahren und 1.685.924 Frauen. Dies ist ein Zuwachs von knapp $4 \%$, gemessen an der Gesamtbevölkerung bei den Männern und ein Zuwachs von etwas über $4 \%$ bei den Frauen, ebenfalls gemessen an den Anteilen der Gesamtbevölkerung in Deutschland (vgl.www.dosb.de).

Diese Zahlen belegen zwar den grundsätzlichen Zuspruch, den Seniorensport erhält, doch die Zahlen geben weder Informationen über mögliche passive Mitglieder noch Informationen über die Sportarten, die auf Breitensportniveau betrieben werden. Umso wichtiger erscheint daher eine genauere Analyse der Gründe, warum manche Senioren im Alter mit dem Sporttreiben beginnen und manche nicht. Auch die Gruppe der Nicht-Mitglieder sollte genauer beleuchtet werden. 


\subsection{Förderungsprogramme für Seniorensport des Deutschen Olympischen Sportbundes (DOSB)}

Die Orientierung des DOSB in Richtung einer gezielten Förderung des Seniorensports beginnt mit einem Rahmenplan vom Mai 1998. Dort werden hauptsächlich drei Kernfragen behandelt, welche die zu planenden weiteren Programme oder Projekte spezifizieren sollen. Diese Vorabfragen lauten folgendermaßen:

- „Welche Vorerfahrungen werden von den Teilnehmern/Teilnehmerinnen erwartet?"

- „Welches Teilnahmemotiv steht bei dem Angebot im Vordergrund?“

- „An welche Altersgruppen wendet sich das Angebot vorwiegend?“ (www.dosb.de: Rahmenplan: 4)

Außerdem sind im Rahmenplan weitere mögliche Ideen zur Förderung des Seniorensports aufgelistet, wie z.B. Informations-Veranstaltungen, Wettbewerbe, Seniorenfreizeiten oder -reisen, Schnupperkurse, Werbekampagnen oder spezielle Seniorentage.

Es wird versucht, diese bisher sehr allgemein formulierten Ziele oder Möglichkeiten zur Förderung des Seniorensports in den folgenden Jahren innerhalb differenzierter Projekte auf unterschiedliche Art und Weise umzusetzen.

Das erste langfristige, zielgerichtete Projekt zum Thema Seniorensport ist das Programm „Richtig fit ab 50“ (www.dosb.de), welches im Zeitraum von Juli 2003 bis Juli 2006 anzusiedeln ist. Die Zielgruppe sind 50- bis 60-jährige Wiedereinsteiger oder Neuanfänger. Als Kernfrage wird folgendes Ziel 
formuliert: Wie können Ältere (ab 50 Jahren) zu (mehr) sportlicher Betätigung motiviert werden?

Als Annäherung zur Beantwortung genau dieser Frage werden zwölf Strategien entwickelt:

1.) „Die richtige Ansprache" $=>$ Seniorensport anders betiteln, damit der Name nicht direkt abschreckend wirkt und sich die Teilnehmenden nicht automatisch alt fühlen

2.) "Inhaltlich vielfältig und abwechslungsreich“ $\Rightarrow>$ eine Mischung aus klassischen Sportarten wie laufen oder schwimmen in Kombination mit neuartigen sportlichen Betätigungen wie bspw. Yoga, Zumba oder Entspannung

3.) „Kompetent betreut“ => gute qualifizierte Übungsleiter und Übungsleiterinnen, Fortbildungen, Lizenzerwerb

4.) "Das Motiv Gesundheit" => starkes Motiv, da die eigene Gesundheit den meisten Menschen am Herzen liegt

5.) „Das Motiv der Leistungssteigerung“ $=>$ auch im Seniorensport ist der Wettkampfaspekt bzw. das Erreichen einer bestimmten Leistung noch wünschenswert

6.) „Neue Erkenntnisse und Erfahrungen ermöglichen“ => geistige Fitness, Informationsveranstaltungen zum Thema Ernährung, Alterserscheinungen etc.

7.) „Spaß und Geselligkeit im Vordergrund“ => Soziale Erlebnisse im Gruppensport (z.B. Essen gehen, Weihnachtsfeier etc.)

8.) "Sport vor der Haustür" => Je kürzer der Weg zum Sport, desto eher wird das Angebot wahrgenommen (z.B. in Kindergärten, Gemeinden, VHS)

9.) „Ein schönes Ambiente“ => Wohlfühlfaktor in den Räumlichkeiten 
10.) „Zeitlich flexibel“ => flexible Trainingszeiten, z.B. personal trainer

11.) „Erst schnuppern, dann binden“ => Schnupperwochen, Kurzmitgliedschaften, keine direkte Vereinsbindung zum Einstieg

12.) "Netzwerken“ => Kooperationen zwischen Sportvereinen, Gemeinden etc.

(www.dosb.de: Broschüre ,Richtig fit ab 50^)

Weitere Projekte, welche jedoch grundsätzlich ähnliche Ziele implizieren und lediglich unterschiedliche Teilaspekte betonen, sind beispielsweise die Initiativen zur ,Sturzprävention' oder ,Bewegungsangebote 70+'. Bei der Bundesinitiative zur Sturzprävention geht es, wie der Name bereits vermuten lässt, darum, die Anzahl der Stürze (mit Verletzungsfolgen) zu reduzieren, indem ein Programm zur Sturzprävention entwickelt wird. Pro Jahr stürzen ca. vier bis fünf Millionen ältere Menschen, woraus etwa 200.000 bis 250.000 Knochenbrüche und damit verbundene Krankenhausaufenthalte resultieren (vgl. Broschüre vom DOSB unter www.richtigfitab50.de). Die Notwendigkeit dieser Initiative wird also durch diese Zahlen deutlich.

Bei den Bewegungsangeboten für die Zielgruppe der Älteren von 70 Jahren und aufwärts ist der Grundgedanke sehr ähnlich zu den Projekten für jüngere Senioren. Der Unterschied liegt hauptsächlich in der Logistik, d.h. wenn die Hochaltrigen nicht mobil genug sind, um zu einer Sportstätte zu gelangen, so sollte es stets die Möglichkeit geben, sportliche Betätigung und Bewegung an Ort und Stelle, wie beispielsweise im Altenheim oder zu Hause, durchzuführen. Die Notwendigkeit für solche Maßnahmen zeigt sich in den Zahlen der Hochaltrigen im weltweiten Vergleich: Deutschland ist das Land mit dem vierthöchsten Durchschnittsalter nach Japan, Italien und der Schweiz (vgl. Broschüre vom DOSB unter www.richtigfitab50.de).

Zwei weitere jüngere Projekte spezialisieren sich auf noch deutlicher definierte Zielgruppen. Im ,Bewegungsnetzwerk 50plus', welches von Januar 2010 bis März 2012 lief, geht es insbesondere um die Vernetzung einzelner Sportanbieter, wie z.B. von Vereinen, Kommunen, Kirchengemeinden, Gesundheitsorganisationen oder anderer sozialer Einrichtungen. Unter 
anderem resultiert aus dieser Organisation auch ein Projekt, welches durch den Badischen Sportbund Nord ins Leben gerufen wurde und welches zum Ziel hat, ältere Migrantinnen und Migranten für das Sporttreiben zu gewinnen. Hierbei handelt es sich um die Eingliederung Älterer mit Migrationshintergrund in Schwimmkurse (mit gesondertem Frauenschwimmen) oder andere Sportgruppen. Darüber hinaus werden von den Migrantinnen und Migranten ebenfalls soziale Angebote angenommen, welche über die Sportkurse hinausgehen, wie z.B. Deutschkurse, geselliges Beisammensitzen bei Kaffee und Kuchen oder Ausflüge in die Umgebung. Der Vorteil dieser Eingliederung in die deutschsprachigen Sportkurse ist eine schnelle Zunahme an Vokabular durch die Möglichkeit, die deutsche Sprache auch in der Freizeit zu sprechen anders als in der vertrauten Gruppe mit gleichsprachigen Migrantinnen und Migranten (vgl. www.richtigfitab50.de).

Das jüngste, aktuell noch laufende Projekt des DOSB lautet ,AUF (Aktiv Und Fit) Leben' und ist für eine Laufzeit von Januar 2013 bis Juni 2015 geplant. Es besteht aus zwei Teilprojekten, zum einen realisiert durch den Deutschen Turnerbund (DTB) und zum anderen durch den Landessportbund Berlin (LSB). Die Zielgruppen des DTB sind einerseits hochaltrige Menschen und andererseits deren Angehörige oder andere pflegende Personen. Die Zielgruppe des LSB umfasst Menschen in der Übergangsphase von der Berufstätigkeit in den Ruhestand.

Der DTB legt seinen Schwerpunkt auf die Arbeit mit Menschen, die an Demenz erkrankt sind, und deren Angehörige. Die pflegenden Angehörigen sollen mithilfe von Bewegungsangeboten die Möglichkeit zum Erfahrungsaustausch bekommen und einerseits durch Bewegung Entlastung erfahren, andererseits durch Gespräche psychischen Rückhalt erleben.

Ein Kernziel des Projekts des LTB ist zunächst die Kontaktaufnahme zu älteren Beschäftigten in Betrieben, um diese gezielt an Sportangebote heranzuführen, sollten diese noch sportlich inaktiv sein. Zudem gilt es als wünschenswert, Sportvereine mit Betrieben zu vernetzen, damit Betriebsangehörige schon vor dem Ruhestand auf mögliche sportliche Betätigungen aufmerksam werden. So 
wird auch die Gesundheitskompetenz der älteren Beschäftigten angesprochen und verändert (vgl. Flyer vom DOSB unter www.richtigfitab50.de).

Trotz der zahlreichen Seniorensportprogramme, die sich in den letzten Jahren entwickelt haben und sich in Zukunft noch entwickeln werden, gibt es immer noch eine Vielzahl von Seniorinnen und Senioren in Deutschland, welche sich nicht sportlich betätigen. Auch das theoretische Wissen über Motivationskonzepte und die demographische Veränderung produzieren nicht automatisch einen Masterplan für die Sportentwicklung in Deutschland. Vielmehr muss individuell beleuchtet werden, was für eine Sportpartizipation ausschlaggebend sein könnte und wie diese Ergebnisse umgesetzt werden können. Außerdem ist die richtige Vermarktung des Konzeptes des Seniorensports von hoher Bedeutung, denn nur wenn die Seniorinnen und Senioren erfahren, dass es Seniorensportgruppen gibt und welches ihre Inhalte sind, können sie den ersten Schritt wagen und Präsenz vor Ort zeigen.

Um genau diesen Aspekt der individuellen Vorstellungen von sportlicher Betätigung anhand qualitativer Interviews mit Seniorinnen und Senioren geht es im folgenden empirischen Teil dieser Arbeit. 


\section{Empirischer Teil}

\section{$6 \quad$ Fragestellung}

Im vorliegenden empirischen Teil folgt nun die Beschreibung der empirischen Erhebung, die, im Hinblick auf die zuvor beschriebene Theorie, neue Erkenntnisse bzw. Abweichungen oder Bestätigungen respektive bestehender Theorien darlegen soll. Wie bereits in der Einleitung dieser Arbeit erwähnt, zielt die empirische Erhebung darauf ab, spezifische Antworten auf die gegebenen Fragestellungen herauszuarbeiten.

Die allgemeine Fragestellung ergibt sich zum einen aufgrund der aktuellen demographischen Entwicklung (vgl. Kapitel 5), welche deutlich widerspiegelt, dass die Bevölkerung immer älter wird. Außerdem fehlt eine konkrete Aufschlüsselung der Mitgliederzahlen innerhalb der Landessportbünde im DOSB. Diese Zahlen berücksichtigen weder inaktive bzw. passive Mitglieder, noch geben sie Auskunft über die Motive, welche die Aktiven zum Sporttreiben motiviert. Zudem fehlen Informationen über Nicht-Mitglieder, also ihre Gründe, wieso sie nicht Teil einer wachsenden Zielgruppe sind, welche im Seniorenalter Sport treibt. Zum anderen legen die meisten Studien, die sich mit Seniorensport befassen, ein besonderes Augenmerk auf aktive Seniorensportler/innen, welche bereits den Großteil ihres Lebens mit aktivem Sporttreiben verbracht haben (vgl. Kapitel 4.5). Das Erkenntnisinteresse dieser Arbeit liegt aber in folgender Hauptfragestellung: Wie kommt es, dass manche Menschen im Seniorenalter (60 Jahre und älter ${ }^{2}$ ) mit dem Sporttreiben beginnen, während andere Menschen ähnlichen Alters inaktiv bleiben?

Des Weiteren sind folgende Fragestellungen von Interesse: Was bewegt Menschen zu der Entscheidung, Sport zu treiben oder umgekehrt, keinen Sport zu treiben? Wie begründen sie ihr Sporttreiben oder ihr Desinteresse an Sport? Welches sind die Motive der Sporttreibenden für einen aktiven Alltag? Wie

\footnotetext{
${ }^{2}$ Die Begründung für die Festlegung der Stichprobe ab 60 Jahren wurde bereits im Theorieteil (S. 13) dargelegt.
} 
präsentieren die Befragten sich selbst in Interviews und welche Definition von Sport setzen sie voraus?

Die Beantwortung dieser und weiterer Fragen soll im Folgenden transparent gemacht werden.

\section{$7 \quad$ Methodologie}

In diesem Kapitel wird die Wahl der Methodologie der empirischen Erhebung vorgestellt. Hier stellt sich die Frage, ob die Erhebung quantitativ oder qualitativ durchgeführt werden soll. Die Entscheidung fällt zugunsten eines qualitativen Verfahrens, welche im Rahmen dieses Kapitels begründet wird.

Auf eine detaillierte theoretische Auseinandersetzung mit qualitativer und quantitativer Sozialforschung wird mangels Relevanz für diese Arbeit jedoch verzichtet und es wird lediglich auf die behandelnden Textpassagen in Diekmanns Werk „Empirische Sozialforschung“ $\left(2010^{4}\right)$ verwiesen.

In den Vorüberlegungen spricht alles für eine qualitative Vorgehensweise: Während in der quantitativen Forschung Hypothesen an einer umfangreichen Stichprobe überprüft werden, wirkt die qualitative Forschung „hypothesengenerierend“ (Engels 2004: 77) und ermöglicht dank einer kleineren Stichprobe zusätzlich einen Fokus auf den Einzelfall (vgl. Rosenthal 2005).

Flick (1996) nennt drei Hauptziele qualitativer Forschung: „Erstens die Erfassung subjektiver Sichtweisen, zweitens die Erforschung der interaktiven Herstellung sozialer Wirklichkeiten und drittens die Identifikation der kulturellen Rahmungen sozialer Wirklichkeiten“ (19). Dies bedeutet, dass versucht wird, die Interaktion von Menschen im sozialen Kontext und deren Handlungsweisen und -auswirkungen zu untersuchen und zu verstehen. Daraus ergibt sich eine sinnhafte Struktur menschlichen sozialen Handelns (vgl. Froschauer \& Lueger 
2003). Im Laufe des Interviews entsteht die so genannte ,narrative Identität'. Diese wird laut Lucius-Hoene \& Deppermann (2002) als „motiviertes kommunikatives Produkt" (11) verstanden. Des Weiteren wird die ,narrative Identität' als die Art und Weise charakterisiert, „wie ein Mensch in konkreten Interaktionen Identitätsarbeit als narrative Darstellung und Herstellung von jeweils situativ relevanten Aspekten seiner Identität leistet" (Lucius-Hoene \& Deppermann 2002: 75).

Die Generierung der Hypothesen erfolgt grundsätzlich erst am Ende der Forschung, um möglichst unvoreingenommen in den Forschungsprozess zu starten. Aufgrund der deutlich kleineren Stichprobe im Vergleich zur quantitativen Sozialforschung wird der Fokus stark auf das Subjekt gelegt, welches im Rahmen von Interviews die Möglichkeit bekommt, einen langen Erzählfluss zu produzieren. Somit kann bei der Subjektbezogenheit „stärker in die Tiefe" gegangen werden und das statistische Material rückt somit in den Hintergrund (Diekmann 20104: 532). In der qualitativen Forschung wird grundsätzlich davon ausgegangen, „dass die zu Grunde liegenden latenten [im Hintergrund ablaufenden] Sinnstrukturen bereits anhand des Einzelfalls bestimmt werden können und eine objektive Gültigkeit über diesen Fall hinaus haben" (Helfferich 20052: 153).

Außerdem wird in der qualitativen Sozialforschung ein zusätzliches Augenmerk auf das Verständnis sozialen Handelns und sozialer Milieus gelegt. Dies spiegelt sich treffend in der Grundannahme interpretativer Sozialforschung wider: „Menschen [handeln] auf der Grundlage ihrer Deutungen der sozialen Wirklichkeit [...] und [stellen] diese Wirklichkeit nach bestimmten sozialen Regeln immer wieder neu interaktiv handelnd her [...]" (Rosenthal 2005: 39).

Qualitative Forschung geht also sehr viel mehr in die Tiefe als quantitative Sozialforschung, bei der das Augenmerk deutlich auf „Aussagen über Verteilungen und Repräsentativität ihrer Ergebnisse" sowie „Verallgemeinerungen basierend auf Häufigkeiten“ gelegt wird (Rosenthal 2005: 26). 
Konkret heißt dies für die durchgeführte Erhebung, dass statt Aussagen über Motive oder subjektive Entscheidungsprozesse, wie sie mithilfe der qualitativen Forschung erhoben werden können, im Rahmen einer quantitativen Analyse Aussagen über Häufigkeiten, also die Anzahl der Sporttreibenden und ähnliche vorgegebene statistische Werte getroffen werden könnten. Dies ist jedoch nicht das Anliegen der gewählten Fragestellung, weshalb der Fokus auf die qualitative Sozialforschung gelegt wird.

Aufgrund des Prinzips der Offenheit in der qualitativen Sozialforschung wird die Stichprobe, also die zu interviewende Anzahl der Personen, nicht vorab festgelegt. Erst im Laufe des Forschungsprozesses, wenn durch weitere Interviews keine neuen Erkenntnisse mehr gewonnen werden können, gilt die Stichprobe als „saturiert" oder „gesättigt" (Helfferich 2005: 155). Je mehr Fälle untersucht werden müssen, desto weniger umfangreich gestaltet sich deren Analyse, sodass eine hohe Stichprobe in der qualitativen Sozialforschung eher nachteilig wirkt. Die spätere Hypothesengenerierung sowie die offene Forschungsfrage tragen ebenfalls zum Prinzip der Offenheit bei. Hiermit erklären sich der Forscher oder die Forscherin automatisch bereit, sich auf das empirische Feld einzulassen, diesem offen zu begegnen, um dadurch neue Erkenntnisse zu erlangen und ggf. ihr vorhergehendes Wissen in den Hintergrund zu stellen bzw. zu modifizieren (vgl. Rosenthal 2005: 48). Demnach erwartet das Prinzip der Offenheit von den Forschenden, „beobachtete Tatbestände nicht vorschnell unter bekannte Kategorien zu subsumieren“ (Gläser \& Laudel 2006: 27-28).

Als negativen Aspekt bezüglich der Anforderungen an qualitative Interviews kritisiert Helfferich $\left(2005^{2}\right)$ die Tatsache, dass die Interviewer/innen all diese Dinge gleichzeitig leisten müssen: „Vertrautheit herstellen und gleichzeitig Fremdheit annehmen, offen sein und gleichzeitig strukturieren, sich als ,naiv' und als ,kundig' darstellen etc. - das können sie [die Interviewer] natürlich nicht" (Helfferich 20052: 9). Hier ist für die Interviews in dieser Arbeit als kritisch anzumerken, dass ein Teil des Vorwissens zu theoretischen Hintergründen nie komplett ausgeblendet werden kann. Dennoch ist der vorangehende Theorieteil in seiner Gesamtgestaltung erst im Anschluss an die qualitative Erhebung 
verfasst worden, um mit einem möglichst unvoreingenommenen theoretischen Wissen in das Forschungsfeld zu starten. Der kleine Anteil an bereits vorhandener Theoriekenntnis wurde im Forschungsverlauf in den Hintergrund gestellt und, so gut es ging, ausgeblendet.

\section{Erhebungsmethode}

Nachdem die Entscheidung für die qualitative Forschungsmethode gefallen ist, muss die Art der Erhebung spezifiziert werden. Dazu werden drei Optionen geprüft: ein Interview (mit Leitfaden oder narrativ, also ohne Leitfaden), eine Gruppendiskussion oder eine teilnehmende Beobachtung. Letztere wird als nicht geeignet angesehen, da dort nicht ausreichend Informationen vom Individuum selbst herausgefiltert werden können, da keine direkte Gesprächsinteraktion stattfinden würde.

Die Entscheidung fällt primär zugunsten der Durchführung narrativer Interviews:

„Das narrative Interview ist eine aus wissenschaftlichem Interesse herbeigeführte und methodisch restringierte Kommunikation, die der Datengewinnung dient. Sie fällt in dieser Form aus dem gewohnten Rahmen alltäglicher Verständigungsformen heraus und bildet ein eigenständiges kommunikatives Verfahren mit besonderen Regeln, Bedingungen, Möglichkeiten und Rollen“ (Lucius-Hoene \& Deppermann 2002: 78).

Die Vorteile des narrativen Interviews gegenüber dem Leitfadeninterview sind hauptsächlich der Grad der Offenheit und das Ausbleiben von festgelegten zeitlichen Limitationen. Im narrativen Interview bleibt den Interviewten selbst überlassen, über welche Dinge sie sprechen möchten und wie sie ihre autonome Erzählung gestalten, also für sie individuell Relevantes ausdrücken wollen (vgl. Lucius-Hoene \& Deppermann 2002). Nach der vom Forscher oder der Forscherin gestellten erzählgenerierenden Eingangsfrage folgt ein autonom gestalteter Hauptteil seitens der Befragten. Hier erzählen diese frei und ohne jegliche Zwischenfragen (vgl. Küsters 2006). 
Im Rahmen dieser Erhebung wird ebenfalls ein Interview in Form einer Gruppendiskussion geführt und bildet damit eine Ausnahme in der Wahl der Erhebungsmethode. Die Gründe hierfür werden im Verlauf der Arbeit dargelegt (vgl. Kapitel 9.3.1).

Bevor die ersten Interviews geführt werden können, bedarf es einer intensiven Vorbereitung, sowohl auf das thematische Feld bezogen als auch auf die Interviewführung an sich. Daher sind Übungen zur Durchführung eines narrativen Interviews unumgänglich und sollten bereits vor der ersten Kontaktaufnahme erfolgt sein. Die erste Kontaktaufnahme beinhaltet in den meisten Fällen bereits die Vereinbarung des Interviewtermins und des -ortes (vgl. Rosenthal 2005).

Im Verlauf des Interviews sind die Befragten drei Arten von Zwängen ausgesetzt: dem Kondensierungszwang, dem Detaillierungszwang und dem Gestaltschließungszwang (vgl. Lucius-Hoene \& Deppermann 2002: 36).

Der Kondensierungszwang beschreibt das interne Dilemma der Interviewten, was die Auswahl der zu erzählenden Geschichten, Themen oder Aspekte angeht. Die Interviewten selektieren also die für sie relevanten Punkte vor, zum einen bewusst, zum anderen unbewusst. Oftmals unterbrechen die Interviewten den Erzählfluss, um spontan erinnerte Geschichten einfließen zu lassen.

Der Detaillierungszwang beschreibt das notwendige Vermitteln von ausreichend Informationen seitens der Interviewten, damit ein Verständnis für den Forschenden ermöglicht wird. So kommt es häufig vor, dass die Interviewten etwas weiter ausholen müssen, um ihre Geschichten verständlich und nachvollziehbar zu übermitteln.

Der Gestaltschließungszwang beschreibt die Notwendigkeit der abgerundeten Erzählung, um einen Gesamtzusammenhang herstellen und die „Bedeutung des Gesamtereignisses sichtbar machen“ zu können (Lucius-Hoene \& Deppermann 2002: 36).

Mit Ausnahme von Gestik, Mimik und unterstützenden Kommunikationsfloskeln des aktiven Zuhörens konzentriert sich der Forscher oder die Forscherin nebenbei auf das Verfassen von Stichpunkten zu angesprochenen Themen der 
Befragten. Diese dienen dem erzählgenerierenden Nachfrageteil im Anschluss an die Haupterzählung. Hier stellt der Forscher oder die Forscherin den Interviewten Fragen zu bereits erwähnten Themen, um noch detailliertere Erzählungen zu provozieren (vgl. Rosenthal 2005). Im Hauptteil des Interviews haben also die Befragten das so genannte "monologische Rederecht" (Helfferich 20052: 24). Nach Schütze (1977) sind die Rollen des Interviewers und der Interviewten in genau diesem Teil daher auch „asymmetrisch, [denn] die Interviewenden haben nur den Part, die Erzählperson zu stützen, und halten sich zurück. Die strikte Asymmetrie dämpft die Kommunikation, [vielmehr die Interaktion], die Erzählperson könnte auch ein Selbstgespräch halten. Sie ist mehr eine Produzentin als eine Partnerin im Interview" (Helfferich 2005²: 31).

Während des Interviews sollte seitens der Befragenden stets davon abgesehen werden, die Interviewten bereits in denkbare Kategorien zu katalogisieren, um so vorschnellen Vereinfachungen aus dem Wege zu gehen. Wichtig für das gelungene Interview ist zudem eine angenehme Gesprächssituation: „Nicht die Fragetechnik läßt ein Gespräch gelingen, sondern die Herstellung einer konstruktiven und vertrauensvollen Gesprächsatmosphäre" (Froschauer \& Lueger: 75-76).

Im Anschluss an das Interview, welches stets aufgezeichnet werden sollte, erfolgt die Transkription - in diesem Fall nach den Regeln von Gabriele Rosenthal (2005), (siehe Anhang, Seite 170). „Für die Tonbandaufzeichnung spricht vor allem, dass jede handschriftliche Protokollierung mit gewaltigen Informationsverlusten und -veränderungen verbunden ist" (Gläser \& Laudel: 152). Nichtsdestotrotz sollte auf handschriftliche Spiegelstriche während des Interviews nicht verzichtet werden, um einen Überblick für das spätere erzählgenerierende Nachfragen zu behalten (vgl. Rosenthal 2005). Eine Transkription dient der leichteren Auswertung und Interpretation der Daten. "Difficult and timeconsuming though transcription is, there really is no satisfactory alternative to recording and fully transcribing qualitative research interviews“ (King 1994: 25, in: Gläser \& Laudel: 188). 


\section{$9 \quad$ Stichprobe}

Im folgenden Kapitel geht es um die exakte Festlegung und Definition der Stichprobe, das heißt der zu interviewenden Seniorinnen und Senioren. Nach der Beschreibung der Auswahlkriterien wird auf die Rekrutierung der Zielgruppe eingegangen und abschließend werden die Interviewpartner im Unterkapitel ,Fallauswahl‘ kurz skizziert.

\subsection{Auswahlkriterien}

Für die Durchführung der qualitativen Interviews muss vorab die Stichprobe definiert und festgelegt werden. Vor Durchführung des ersten Interviews werden die ersten Kriterien bzw. Rahmenbedingungen für die Auswahl der Teilnehmerinnen und Teilnehmer formuliert und im Laufe des Forschungsprozesses werden weitere Kriterien bzw. Zielgruppen festgelegt. Die Festlegung auf die Kriterien erfolgt anhand des Anspruchs, eine möglichst ausgeglichene Befragung zu ermöglichen, in der die Verteilungen der Kriterien relativ gleichmäßig ausfallen.

Das erste, wichtigste Kriterium ist das Alter: Die Befragten müssen wenigstens 60 Jahre alt sein, wobei es nach oben keine Grenze gibt. Des Weiteren ist es von hoher Bedeutung, die Stichprobe relativ ausgeglichen zu gestalten, was die Repräsentativität der Geschlechter angeht. Auch die Verteilung der Wohnorte ist von Interesse. Daher werden sowohl Interviews mit Seniorinnen und Senioren aus dem städtischen Bereich Göttingen geführt als auch Menschen aus der ländlichen Region rund um Göttingen befragt.

Nachdem die Fragestellung der Arbeit unter Vorbehalt festgelegt und die Unterscheidung von Nicht-Sportlern und Wiederanfängern ${ }^{3}$ getroffen worden ist, stellt sich nach einigen Interviews heraus, dass es, insbesondere bei den

\footnotetext{
${ }^{3}$ Hier werden die Seniorinnen und Senioren, welche zwar einen aktiven Lebensstil verkörpern, jedoch nicht nach der in dieser Arbeit gegebenen Sportdefinition körperlich aktiv sind, zu den Nicht-Sportlern gezählt, um eine deutliche Abgrenzung zwischen Wiederanfängern und Nicht-Sportlern vorzunehmen.
} 
Wiederanfängern, eine Vielzahl an Untergruppen geben würde. So scheint es sinnvoll zu sein, nicht nur Vereinsmitglieder, sondern auch FitnessstudioBesucherinnen und -Besucher und Individualsportlerinnen und -sportler zu interviewen, um je nach Organisationszugehörigkeit eine breitere Stichprobe erheben zu können. Auch die Vereinsmitglieder können in weitere Untergruppen, je nach Kursbelegung, unterschieden werden.

\section{$9.2 \quad$ Rekrutierung}

Die erste und auf den ersten Blick einfachste Variante der Kontaktaufnahme zu Interviewpartnerinnen und -partnern, welche sportlich aktiv sind, ist der Weg über Vereine. Hier muss kritisch angemerkt werden, dass Vereine lediglich eine, in ihrer Organisationsform ähnliche Möglichkeit des Sporttreibens anbieten, nämlich die Form des organisierten Gruppenerlebnisses. Aufgrund der lokalen Bedingungen wird zunächst nach Vereinen in der Stadt Göttingen gesucht, welche explizit Seniorensport als eigene Sparte ausweisen.

Hier kristallisieren sich drei Vereine heraus - zwei eher kleinere und ein großer Verein in Göttingen. Zunächst erfolgt eine erste Kontaktaufnahme via E-Mail, adressiert an die jeweilig auf der Internetseite angegebenen Gruppenleiterinnen und -leiter der Seniorensportsparte oder direkt an Kursleitende der jeweiligen Gruppen. Hier wird in den meisten Fällen ein Termin vereinbart, an dem der Kurs stattfindet und daher eine direkte Kontaktaufnahme zu den Seniorinnen und Senioren möglich ist. Lediglich in einem Verein findet ein Treffen mit der Verantwortlichen für Seniorensport statt, bevor es die offizielle Einwilligung des Vereins im Hinblick auf die Studie geben wird.

Nachdem die Formalitäten erfolgreich abgeschlossen sind, besteht die Möglichkeit des direkten Kontaktes zu den jeweiligen Seniorensportgruppen. Die Kontaktaufnahme findet stets vor Beginn der entsprechenden Kurse statt und beinhaltet eine kurze Vorstellung meinerseits sowie eine kurze Einführung in die Thematik des Forschungsvorhabens. Wenn die Kriterien für die Zielgruppe der zu interviewenden Freiwilligen eingegrenzt sind, spaltet sich die 
Gruppe in potenzielle Zielpersonen und Seniorinnen und Senioren, die nicht in Frage kommen, da sie entweder noch nicht über 60 Jahre alt sind (sehr selten) oder keine Wiederanfänger sind, sondern mehr oder weniger ihr ganzes Leben lang Sport getrieben haben.

Unter den Seniorinnen und Senioren, die sich eigenständig als passend in die Zielgruppe einstufen, erklären sich fast alle zu einem Interview bereit. Zwar zeigen sich einige skeptischer und vorsichtiger als andere, im Großen und Ganzen jedoch ist ein hohes $\mathrm{Maß}$ an Hilfsbereitschaft und Offenheit zu erkennen.

Die Kooperationsbereitschaft des Großteils der Interviewpartnerinnen und partner ist als sehr positiv hervorzuheben. Nach Beendigung des Vorstellungsteils werden entweder direkt Interviewtermine vereinbart oder zunächst Telefonnummern ausgetauscht, um einen Termin nach einer Bedenkzeit von ein paar Tagen per Telefon zu klären. Der Anruf erfolgt im Schnitt etwa drei Tage nach dem ersten persönlichen Kontakt. So wird einerseits eine Bedenkzeit gewährleistet, andererseits geraten der erste Eindruck und das Forschungsvorhaben nicht in Vergessenheit.

Alle Seniorinnen und Senioren, welche eine Telefonnummer hinterlassen haben, erklären sich zu einem Interview bereit. Inhalte der Telefongespräche sind hauptsächlich die Terminfindung sowie die Vereinbarung eines Ortes. In den meisten Fällen wird das eigene Domizil der Interviewten als Ort angeboten, in wenigen anderen Fällen werden entweder Cafés oder Räume der GeorgAugust-Universität Göttingen ausgewählt. Kleine Unsicherheiten oder Zweifel werden bereits in den Telefongesprächen diskutiert und weitestgehend eliminiert.

Um die Gruppe der Nicht-Sportler zu rekrutieren, bedarf es interessanterweise ähnlicher Strategien: Auch hier wird der erste Kontakt über einen Verein hergestellt, der nicht nur sportliche, sondern auch kulturelle Angebote, wie etwa das Singen in einem Chor oder schauspielerische Tätigkeiten in einer Theatergruppe offeriert. Zwar sind die meisten Teilnehmerinnen und Teilnehmer dieser kulturellen und sozialen Angebote zudem in sportlichen 
Gruppen aktiv, doch eine kleine Gruppe kristallisiert sich als sportlich inaktiv heraus und ist schnell bereit für ein narratives Interview.

Lediglich ein einziges Interview findet nicht statt, weil der Interviewpartner den Interviewtermin vergessen hat. Aufgrund der hohen Anzahl an geführten Interviews mit Nicht-Sportlern wird auf eine Neuterminierung verzichtet und alleinig eine kurze Notiz hinterlegt.

Die Kontaktaufnahme in den Fitnessstudios erfolgt letztlich auf einem ähnlichen Weg, wobei hier ein erstes Treffen mit den verantwortlichen Trainern vonnöten ist. Nachdem auf eine erste E-Mail, gerichtet an fünf Fitnessstudios in Göttingen, nicht reagiert worden ist, scheint der persönliche Kontakt die beste Lösung zu sein. Die Fitnessstudios werden anhand ihres Internetauftrittes ausgewählt, welcher in allen Fällen entweder die Rubrik ,Rehasport' oder ,Seniorensport' beinhaltet. Den Trainerinnen und Trainern gefällt das Forschungsprojekt und daher zeigen sie sich schließlich sehr hilfsbereit und kooperativ.

In einem Fitnessstudio wird der direkte Kontakt der Forscherin mit den potenziellen Probanden erlaubt. Es erfolgt ein kurzes Vorstellen meiner Person und meines Forschungsvorhabens. Hier passt nur ein Herr in meine Zielgruppe, da alle anderen schon seit vielen Jahren Sport treiben und nicht erst im Seniorenalter wieder damit begonnen haben.

In zwei weiteren Fitnessstudios wird der direkte Kontakt zu den möglichen Seniorinnen und Senioren nicht gestattet, sondern mittels der Trainerin auf das Forschungsprojekt aufmerksam gemacht. Bei Interesse werden meine Telefonnummer und E-Mail-Adresse weitergegeben, sodass sich Interessierte theoretisch jederzeit melden können. In der Praxis klappt dies jedoch nicht, obwohl die Trainerinnen sehr ambitioniert und befürwortend dem Projekt gegenüber erscheinen.

Eine weitere Gruppe von Interviewpartnerinnen und -partnern wird anhand des ,Schneeballsystems' rekrutiert. „Beim Schneeballsystem werden Personen, die man kennt, gefragt, ob sie Personen kennen, die bestimmte Kriterien für die Interviewteilnahme erfüllen, oder ob sie Personen kennen, die wiederum Personen kennen etc." (Helfferich 2005²: 156). Dies bedeutet, dass Freunde 
und Bekannte von dem Forschungsprojekt erfahren und somit auch Seniorinnen und Senioren aus ländlicheren Regionen aus der Umgebung von Göttingen erfasst werden können.

Hier wird nach Absprache die Telefonnummer der möglichen Interviewpartnerinnen und -partner direkt an die Forscherin weitergegeben und daraufhin folgen die telefonischen Terminabsprachen und das Beantworten eventueller Rückfragen.

Darüber hinaus besteht die Möglichkeit, das Forschungsvorhaben in einer lokalen Vereinszeitschrift abdrucken zu können, woraufhin sich ebenfalls ein paar Freiwillige melden. Auch hier folgt eine Kontaktaufnahme per Telefon und Interviewtermine und Orte werden festgelegt.

Im Großen und Ganzen ist das Rekrutieren der Interviewpartnerinnen und Interviewpartner zwar einerseits ein aufwendiges Vorgehen, das jedoch andererseits in den meisten Fällen direkt mit Zusagen der Interessierten belohnt wird. Nur wenige Seniorinnen und Senioren, welche potenziell zur Zielgruppe gehören, sind nicht bereit, sich für das Projekt zur Verfügung zu stellen, sodass die Teilnahmebereitschaft insgesamt als sehr positiv hervorgehoben werden kann.

Eine Differenzierung der Interviewten nach Klassen- bzw. Schichtenzugehörigkeit soll in dieser Arbeit nicht vorgenommen werden, denn die Kriterien für eine Bestimmung der Klasse bzw. Schicht in der Sozialforschung unterliegen spätestens seit den 80er Jahren des 20. Jahrhunderts einer kontroversen Diskussion, die einerseits bis zur völligen Negierung von Klassen und Schichten in der heutigen Zeit reicht und andererseits mithilfe geänderter Kriterien eine Schichtstruktur auch in der modernen Gesellschaft konstatiert (vgl. Geißler 2004).

Vor einer Bestimmung der Schichtenzugehörigkeit müssten also die mannigfaltigen Kriterien dafür genau festgelegt werden, zu denen z. B. auch das Konzept des Lebensstils gehört (vgl. Becker \& Schneider 1995).

Vor allem würde aber eine solche Bestimmung voraussetzen, dass die Interviewerin viele persönliche Angaben der Interviewten einfordern müsste, um eine Schichteinteilung möglich zu machen. Doch diesbezügliche Fragen 
könnten die Interviewten abschrecken und in ihrer Offenheit blockieren, weil sie sich möglicherweise in "Schubladen“ gesteckt fühlen würden. Zudem widerspräche diese Vorgehensweise der gewählten Methode des qualitativen Interviews.

\subsection{Fallauswahl}

Die Gesamtstichprobengröße umfasst am Ende 20 Interviewpartnerinnen und Interviewpartner. Davon werden zwölf Interviews mit Frauen und acht Interviews mit Männern geführt.

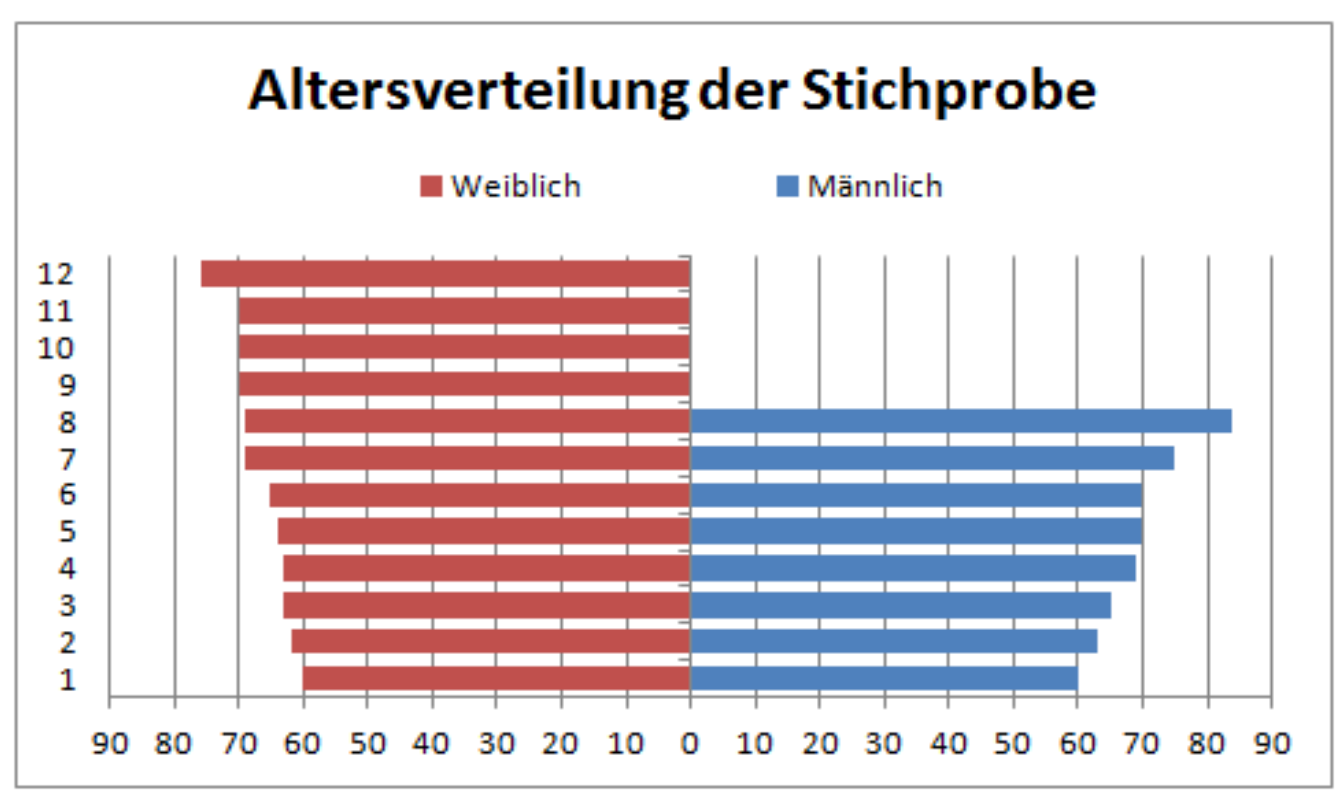

Abbildung 8: Altersverteilung der Stichprobe

Insgesamt werden 13 Sportler und Sportlerinnen und sieben Nicht-Sportler bzw. Nicht-Sportlerinnen interviewt. Unter den 13 sportlich Aktiven gibt es acht Frauen und fünf Männer. Bei den Nicht-Sportlerinnen und Nicht-Sportlern ist die Stichprobe auf drei Herren und vier Frauen begrenzt.

Insgesamt werden 14 Interviews mit Seniorinnen und Senioren aus dem städtischen Bereich Göttingen geführt und folglich sechs Interviews mit 
Menschen aus der ländlichen Region rund um Göttingen. Darüber hinaus können die Sportlerinnen und Sportler in weitere Untergruppen unterteilt werden, welche bei der Rekrutierung zu berücksichtigen sind. So werden acht Seniorinnen und Senioren befragt, die Gymnastik in einem Verein machen, vier Seniorinnen und Senioren, welche im Fitnessstudio aktiv sind, und vier Sportlerinnen und Sportler, die sich individuell sportlich betätigen, nämlich schwimmen, Rad fahren oder walken. In Einzelfällen gibt es zusätzliche Überschneidungen, bei denen die Befragten beispielsweise sowohl im Verein Gymnastik machen, als auch individuell walken oder schwimmen gehen.

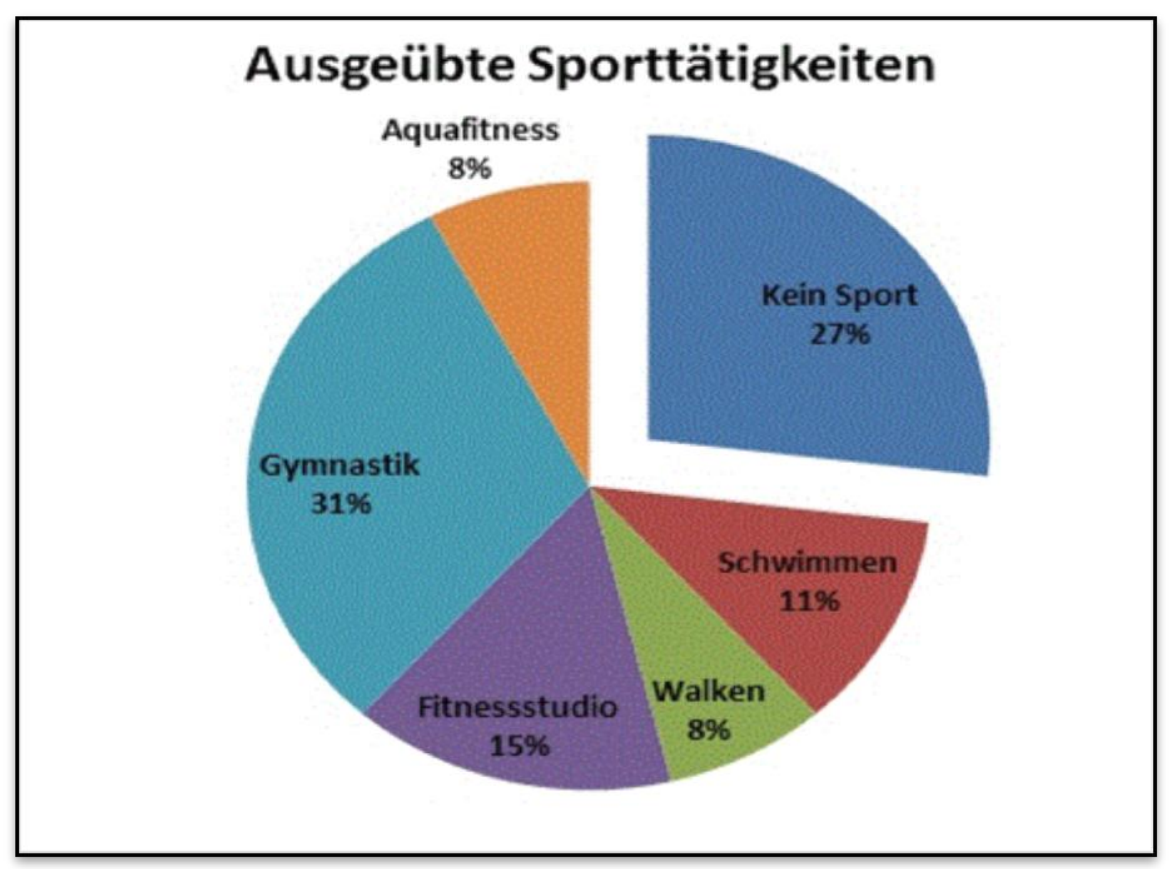

Abbildung 9: Ausgeübte Sporttätigkeiten

Aufgrund des hohen Anteils der Menschen mit Migrationshintergrund an der Göttinger Bevölkerung, ungefähr 19 \% laut Göttinger Statistik Journal von 4/2010 (online: Zugriff am 08.07.2013), wird diese Zielgruppe ebenfalls in die Stichprobe mit eingeschlossen, sodass am Ende die Faktoren der Sportaktivität, des Geschlechts, der Wohnverteilung, der Nationalität, des Alters und der konkreten sportlichen Betätigung berücksichtigt werden.

Warum die Befragung der Migrantinnen lediglich als ein Interview gilt, wird im Folgenden näher erläutert (vgl. Kapitel 9.3.1). 


\section{Interviewdurchführung}

In diesem Kapitel geht es um die Durchführung der qualitativen Interviews, also den Interviewzeitraum, die Orte, an denen die Interviews geführt werden, die Dauer der Interviews, den Ablauf und besondere Vorkommnisse sowie die Teilnahmebereitschaft der Befragten während der Interviews. Das Kapitel schließt mit einem Unterpunkt zum Thema Datenschutz.

\subsection{Zeitraum und Orte}

Die qualitativen Interviews werden im Zeitraum vom 23. April 2012 bis zum 21. Januar 2013 durchgeführt. Die große Mehrheit der aufgenommenen Interviews findet privat bei den Interviewpartnerinnen und Interviewpartnern statt. Lediglich in Einzelfällen wird ein neutraler Ort für das Gespräch gewählt. In diesen Fällen handelt es sich entweder um ein Café, einen Vereinsraum oder Räumlichkeiten der Georg-August-Universität Göttingen.

\subsection{Dauer}

Die Dauer der Interviews variiert von Mal zu Mal deutlich. Während das kürzeste Interview mit exakt 09:00 Minuten auf den ersten Blick deutlich weniger Informationsdichte umfasst, dauert das längste Interview mit 109:10 Minuten fast genau 100 Minuten länger. Im Schnitt dauern die Interviews etwa eine Dreiviertelstunde (ca. 43 Minuten). Die Länge der Interviews lässt sich in den meisten Fällen bereits anhand der Haupterzählung erahnen, da diese bei den umfangreicheren Interviews ebenfalls länger gestaltet ist. Die ausführlichste Haupterzählung umfasst eine Länge von knapp 22 Seiten in der Transkription und die kürzeste Haupterzählung ist lediglich acht Zeilen lang.

Das folgende Diagramm visualisiert die Interviewdauer in Relation zu dem Geschlecht und dem Alter der Befragten: 


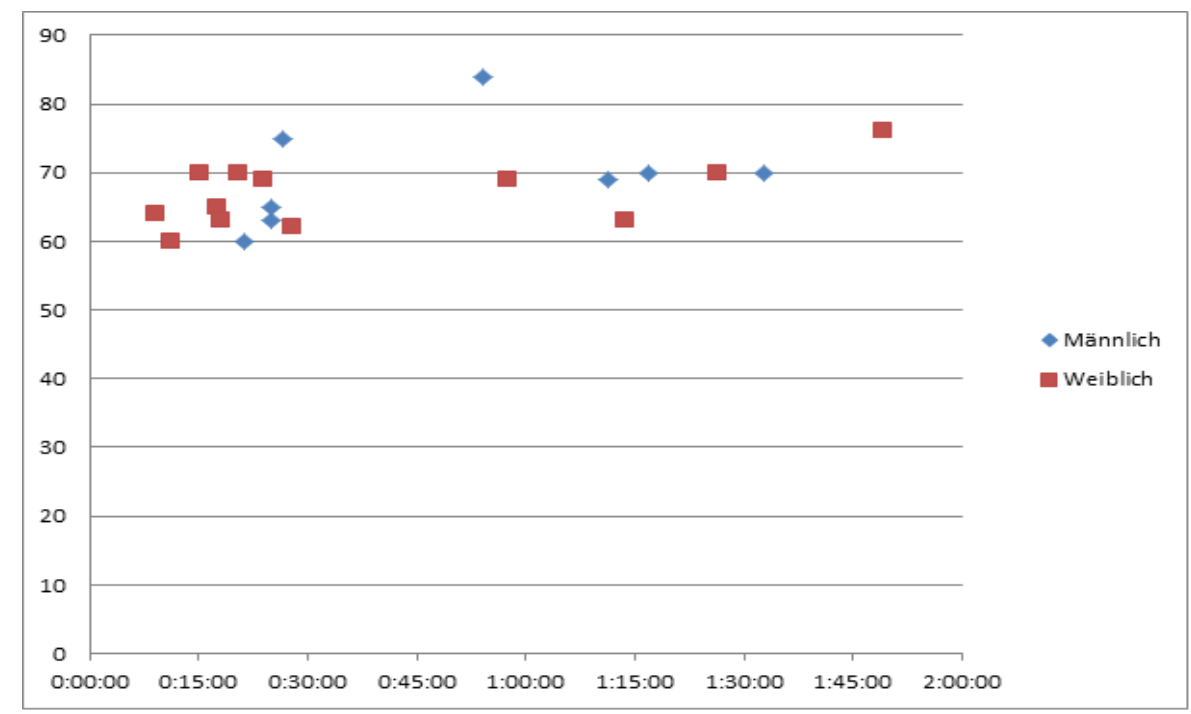

Abbildung 10: Interviewdauer

Hier ist lediglich eine Auffälligkeit hervorzuheben: Die älteste weibliche Befragte gibt das mit Abstand längste Interview. Viele Interviews bewegen sich um eine Zeit von etwa 20 - 30 Minuten. Während die Durchschnittsdauer gar nicht direkt erreicht wird (ca. 43 Minuten), gibt es folglich viele Interviews (8), die deutlich über dieser Zeit liegen. Hier ist die Verteilung von Mann und Frau ausgeglichen - vier Männer und vier Frauen sind hier deutlich über dem Durchschnitt angesiedelt.

\subsection{Ablauf und besondere Vorkommnisse}

Nachdem eine Terminfindung am Telefon oder persönlich stattgefunden hat, beginnt der praktische Teil, nämlich die eigentliche Durchführung der qualitativen Interviews. Die meisten Seniorinnen und Senioren bevorzugen ein Gespräch in ihrer eigenen Wohnung. Die vertraute Umgebung erzeugt bei innen eine gewisse Sicherheit und mildert dadurch die Aufregung, welche die ungewohnte Situation des Interviews hervorruft. So entsteht auch für die Interviewerin eine entspannte Atmosphäre. 
Zunächst wird der Ablauf des Interviews erklärt und überprüft, ob es zur technischen Durchführung noch Fragen gibt. Sollte dies nicht der Fall sein, beginnt das Interview mit Einschalten des Aufnahmegeräts sowie der Formulierung der folgenden Eingangsfrage bzw. Erzählaufforderung, die bei allen Interviews den Einstieg markiert:

„ICh bin an Ihren persönlichen Erfahrungen mit dem Thema Sporttreiben interessiert. Ich möchte Sie bitten, mir all die Erlebnisse aus Ihrem bisherigen Leben zu erzählen, die Ihnen direkt oder indirekt zum Thema Sporttreiben einfallen. Sie können sich dafür so viel Zeit nehmen, wie Sie möchten. Ich werde Sie nicht unterbrechen, mir nur einige Notizen machen und später noch einmal darauf zurückkommen."

In Ausnahmefällen kommt direkt nach der Erzählaufforderung eine Nachfrage seitens der Interviewten, beispielsweise wo sie denn beginnen sollten oder ob alles relevant sei, doch die Mehrheit der Interviewten beginnt sofort vom eigenen Sportverhalten zu erzählen. In den meisten Fällen bleiben die Interviewten sehr auf das Thema fokussiert und fast alle scheinen sich bereits vor dem Interview über ihr (Nicht-)Sportlerleben Gedanken gemacht zu haben. Lediglich ein kleiner Teil der Befragten ist unsicher darüber, was relevant sei, und erzählt daher weniger flüssig und konzentriert. Ein anderer sehr kleiner Teil der Befragten schweift ab und verfängt sich in seltenen Ausnahmen in Anekdoten aus der Vergangenheit, welche jedoch auf den ersten Blick keinen Bezug zum Thema Sporttreiben haben.

Nach Beendigung der Haupterzählung folgt ein Nachfrageteil seitens der Interviewerin. Hier wird an zuvor Erzähltes angeknüpft und weiter in die Tiefe gefragt, in der Hoffnung, mehr Details, Geschichten oder Hintergründe zu erfahren.

Gegenfragen seitens der Interviewten werden in den meisten Fällen entweder kurz beantwortet oder auf den Zeitraum nach dem Interview verschoben. Direkt im Anschluss an das Interview wird das Aufnahmegerät abgeschaltet und darüber gesprochen, wie das Interview empfunden worden sei und ob die Befragten zufrieden mit dem Resultat seien. 
Manchmal folgen nach Abschalten des Aufnahmegerätes vertrauliche Themen, die bewusst nicht in die Aufnahme eingebunden werden sollten. In solchen Fällen werden Notizen gemacht und das Verhalten wird in der Auswertung dennoch analysiert. Diese Zwischenfälle geschehen auch vereinzelt im Verlauf des Interviews. Hier bittet der oder die Interviewte relativ plötzlich um die temporäre Ausschaltung des Aufnahmegerätes. Dies wird selbstverständlich respektiert und schriftlich festgehalten.

In weiteren Fällen beginnen die Interviewten bereits vor dem eigentlichen Beginn des Interviews mit ihren Erzählungen. Hier wird oftmals versucht, die Interviewten zu stoppen und darauf hinzuweisen, dass alles Erzählte relevant für die Aufnahme sei und daher nicht vergeudet bzw. vorweggenommen werden solle.

\subsection{Ablauf und Begründung des Migrantinnen-Interviews}

Nachdem ein erstes Kennenlernen mit der Migrantengruppe in einem Treffpunkt für Menschen mit Migrationshintergrund erfolgt ist, wird schnell deutlich, dass Einzelinterviews mit den anwesenden Frauen nicht in der gewöhnlichen Form erfolgreich durchzuführen wären. Die Deutschkenntnisse der Migrantinnen sind in allen Fällen nicht ausreichend, um sich exakt ausdrücken zu können. Die Frauen wirken außerdem mit ihrer Gruppe sehr vertraut - sie befinden sich in ihrem gewohnten Umfeld mit Freundinnen und Bekannten, oft Gleichgesinnten. Sie außerhalb dieses Umfelds in Einzelinterviews zu befragen, lässt vermuten, dass gar kein Erzählfluss entstehen könnte. Mit exakt dieser Problematik befasst sich auch Claudia Helfferich (2005²):

\footnotetext{
„Nicht von allen sozialen Gruppen kann gleichermaßen erwartet werden, dass sie von sich aus, am Stück und ohne weitere Vorgaben, eine substanziell für die Auswertung notwendige Menge an Erzählung produzieren - dies ist aber Voraussetzung z.B. für narrative Interviews“ (34).
}

Ein Einzelinterview durchzuführen wird also als nicht sinnvoll erachtet. Im Gruppeninterview wird dank aller Anwesenden gegenseitig stets Hilfe 
angeboten. So kommt es oft vor, dass eine Migrantin Wortfindungsschwierigkeiten hat und ein Wort in ihrer Muttersprache sagt, welches eine andere Migrantin ihr dann ins Deutsche übersetzen kann. (Vgl. z.B. Seite 1 der Transkription, Zeilen 11-13.) Um zu vermeiden, dass ohne diese Sicherheit der sprachlichen Unterstützung weniger Dialog zustande käme und die Migrantinnen weniger über ihre Erfahrungen mit dem Sporttreiben erzählten, wird ein Gruppeninterview durchgeführt.

Zusätzlich geben sich die Interviewten gegenseitig Denkanstöße bzw. Themenvorschläge, auf welche die anderen dann reagieren können. (Vgl. z.B. Seite 1 der Transkription, Zeile 29.) So lebt das Interview von seiner eigenen Dynamik; eventuelle Unklarheiten werden direkt in der Gruppe eliminiert und bei Verständnisschwierigkeiten können sich alle gegenseitig helfen und ggf. in der Landessprache nach den richtigen Übersetzungen suchen. (Vgl. z.B. Seite 3 der Transkription, Zeilen 7-15.)

So produziert das Gruppeninterview zwar keine umfassenden Erzählungen von Einzelpersonen, gibt jedoch einen guten Überblick über das Sportverhalten von Seniorinnen mit Migrationshintergrund. Dank der eigenen Dynamik, welche sich im Verlauf des Interviews entwickelt, treten regelmäßig neue Thematiken in den Fokus und eventuelle Missverständnisse können stets durch andere korrigiert werden.

Welche möglichen Nachteile im Zuge einer Gruppendiskussion dennoch entstehen können, wird innerhalb des Kapitels der kritischen Reflexion (Kapitel 11.5) hervorgehoben.

\subsection{Teilnahmebereitschaft der Befragten}

Im Verlauf des Interviews, in der so genannten Haupterzählung, wird schnell deutlich, inwieweit die Interviewten in ihren Erzählpassagen ins Detail gehen bzw. wie viel sie von ihrem Privatleben preisgeben möchten. Hier existieren extreme Unterschiede, welche sich hauptsächlich in der Gesamtlänge der Interviews widerspiegeln: je detaillierter und privater, desto länger das Interview 
und umgekehrt. In den Fällen, in denen es zu Abschweifungen vom eigentlichen Themenbereich kommt, muss die Interviewerin nach einer gewissen Zeit intervenieren, damit das Interview nicht den Fokus verliert.

Die sehr privat und vertraulich erzählenden Interviewten versetzen sich mit ihren Erzählungen oftmals so stark in ihre vergangene Gefühlslage zurück, dass der Eindruck entsteht, sie erlebten die Situationen und Emotionen durch lebhaftes Erzählen erneut. Verdeutlicht wird dies durch Gefühlsausbrüche wie Weinen, Lachen oder Wut und Verständnislosigkeit.

\subsection{Datenschutz}

Bereits während der ersten Kontaktaufnahme, also entweder persönlich oder am Telefon, wird das Stichwort ,Datenschutz' thematisiert. Alle Interviewpartnerinnen und -partner bekommen eine schriftliche Erklärung, dass alle persönlichen Daten anonymisiert werden und alle Inhalte selbstverständlich vertraulich behandelt werden.

Folgendes wird den Interviewten vor dem Interview vorgelegt:

„Ich bin dem Datenschutz verpflichtet und es ist selbstverständlich, dass die auf Tonband aufgezeichneten Interviews nur für wissenschaftliche Zwecke verwendet werden. Persönliche Daten werden bei Veröffentlichungen so verändert, dass eine Identifizierung der Personen nicht möglich ist." (Rosenthal 2005: 90-91)

Hiermit wird den Teilnehmenden versichert, dass in der veröffentlichten Arbeit keine Rückschlüsse auf die Befragten gezogen werden können, da sowohl die Namen der Interviewten und Orte verändert sowie markante Lebensereignisse so abgewandelt werden, dass Außenstehende nicht automatisch Rückschlüsse auf die Person ziehen können. Die Namen der Interviewten sind daher frei erfunden. Lediglich die Befragten selbst könnten sich aufgrund ihrer Erzählung wiederfinden wegen der Zitate in der Veröffentlichung. 


\section{Auswertungsverfahren}

Im folgenden Kapitel geht es um die Methodik, welche zur Auswertung der qualitativen Interviews gewählt wird. Zunächst soll festgelegt werden, ob bei der Auswertung eine Reinform der Analyse oder eine Mischform herangezogen wird.

Ein Problem bei Reinformen ist, dass gewisse Punkte unter Umständen nicht herauskristallisiert werden können. Grundsätzlich existieren drei große Arten von Analyseverfahren, nämlich zum einen die Inhaltsanalyse, welche sich größtenteils mit dem Inhalt der Interviews befasst, das heißt sich auf der WASEbene befindet. Zum anderen gibt es gesprächsanalytische texthermeneutische Auswertungsverfahren, welche sich mehr auf die WIE-Ebene konzentrieren, also auf die Art und Weise des Gesagten achten und Inhalte in den Hintergrund rücken. Eine weitere Methodik der Auswertung ist die Grounded Theory, welche jedoch aufgrund ihrer Detailliertheit aus forschungsökonomischen Gründen in dieser Arbeit nicht weiter behandelt wird. Eine ausführliche Beschreibung der Grounded Theory lässt sich unter anderem in Jörg Strübings (2004) Werk "Grounded Theory. Zur sozialtheoretischen und epistemologischen Fundierung des Verfahrens der empirisch begründeten Theoriebildung" nachlesen.

\subsection{Qualitative Inhaltsanalyse nach Mayring}

„Die Stärke der qualitativen Inhaltsanalyse liegt in ihrem systematischen, regelgeleiteten Vorgehen, mit dem auch große Materialmengen bearbeitet werden können" (Mayring 2010"11: 124).

Die Inhaltsanalyse nach Mayring $\left(2010^{11}\right)$ befasst sich grundsätzlich mit der Analyse von Inhalten einer Kommunikation. Es wird nicht willkürlich und subjektiv frei interpretiert, sondern stets systematisch vorgegangen. So müssen ausnahmslos strikte Regeln der Auswertung eingehalten werden, sodass die Analyse problemlos von Außenstehenden nachvollzogen werden kann. Hierbei 
handelt es sich um die so genannte ,intersubjektive Nachvollziehbarkeit", welche als sozialwissenschaftlicher Methodenstandard vorausgesetzt wird.

Ziel ist es nicht nur, die Inhalte des Textmaterials zu analysieren, sondern auch Absichten der Interviewten herauszufiltern (vgl. Mayring 2010: 11 ff.). Der Hauptschwerpunkt der qualitativen Inhaltsanalyse befasst sich mit dem WAS, also mit den erwähnten Fakten, die bestimmten Kategorien zugeordnet werden können. Dieses Kategoriensystem liegt der Inhaltsanalyse als Methodik zugrunde, indem anhand des vorliegenden Materials Kategorien gebildet werden, welche im Verlauf der Analyse getestet, überprüft und ggf. gestrichen oder ergänzt werden.

Wenn Kategorien bereits vor der Betrachtung des Materials festgelegt werden, handelt es sich hierbei um eine deduktive Kategorienbildung, also die Annahme, dass Kategorien, welche in der Literatur in ähnlichen Studien gebildet wurden, übernehmbar sind für die eigene Studie. Werden die Kategorien erst im Laufe der Analyse anhand des eigenen Materials gebildet und festgelegt, spricht man von induktiver Kategorienbildung. Hier ist wichtig, dass sich die Kategorien nicht auf „Vorab formulierte Theorienkonzepte beziehen“ (Mayring 2010'11: 83).

Einige dieser Unterkategorien werden am Ende der Auswertung zu umfassenden Hauptkategorien zusammengefasst. Hierbei handelt es sich um einen Reduzierungsschritt, bei dem wiederkehrende und sich ähnelnde Aussagen zusammengefasst und gebündelt werden. Dies geschieht mit der Absicht, ein kleineres Kategoriensystem zu erstellen, welches eine bessere Übersicht ermöglicht. Hier entsteht auch die Kapitelauswahl für den weiteren Verlauf des Auswertungsteils.

Bevor die eigentlichen Kategorien gebildet werden können, muss das Material in so genannte „Analyseeinheiten“ (Ebenda: 69) unterteilt werden, also in kurze Abschnitte, die nach und nach systematisch analysiert werden. Nachdem die Analyseeinheit festgelegt worden ist, wird der Textabschnitt paraphrasiert, das heißt mithilfe von Synonymen in andere, sinngleiche Worte umformuliert.

Da bei der qualitativen Inhaltsanalyse sehr systematisch vorgegangen wird, besteht die Gefahr, die eigenen Kategorien nicht mehr zu hinterfragen. Doch 
der Forscher bzw. die Forscherin muss stets den Blick in die Tiefe des Materials beibehalten: „Qualitative Inhaltsanalyse ist immer ein Verstehensprozess von vielschichtigen Sinnstrukturen im Material. Die Analyse darf nicht bei dem manifesten Oberflächeninhalt stehen bleiben, sie muss auch auf latente Sinngehalte abzielen" (Mayring 2010 ${ }^{11}: 32$ ).

Grundsätzlich ist die qualitative Inhaltsanalyse das „einzige Verfahren der qualitativen Textanalyse, das sich frühzeitig und konsequent vom Ursprungstext trennt [durch Paraphrasieren] und versucht, die Informationsfülle systematisch zu reduzieren [...]“" (Gläser \& Laudel 2009: 200). Hierbei ist entscheidend, dass nur die Textpassagen analysiert werden, welche im Hinblick auf das Untersuchungsziel als relevant erkannt werden. Wenn es um das Sportverhalten von Seniorinnen und Senioren geht, dann ist beispielsweise eine Erzählpassage zum Thema der Namensänderung einer Befragten nicht relevant (vgl. Interview mit Frau Oder). Die Nicht-Analyse von gewissen Textpassagen sollte jedoch stets mit Vorsicht behandelt werden, da manche Passagen beim tieferen Einblick doch von Nutzen sein können und ggf. indirekt auf das Untersuchungsziel hinweisen könnten.

Die qualitative Inhaltsanalyse ist charakterisiert durch das Durchführen bestimmter Techniken (vgl. Mayring 2010 ${ }^{11}: 48 \mathrm{ff}$.):

a) „Einbettung des Materials in den Kommunikationszusammenhang“ (48)

Dieser Punkt beschreibt die Wichtigkeit des Kontextes innerhalb einer Kommunikation bzw. in einem Interview. So sollte ein Satz nie isoliert betrachtet werden, sondern stets auf den Gesamtkontext der Passage geachtet werden.

b) „Systematisches, regelgeleitetes Vorgehen“ (48-49)

Die qualitative Inhaltsanalyse ist zwar systematisch, jedoch je nach Textmaterial immer individuell. Grundsätzlich gelten aber die festgelegten Regeln der Textanalyse, welche nicht vernachlässigt werden sollten. Wichtig 
bei diesem Punkt ist die Tatsache, dass jeder Analyseschritt nachvollzogen werden kann, da er die definierten Regeln der Analyse nicht verletzen sollte.

c) „Kategorien im Zentrum der Analyse“ (49-50)

Das Bilden von Kategorien ist eines der Hauptcharakteristika in der qualitativen Inhaltsanalyse und sorgt, wie bereits erwähnt, für die Intersubjektivität. Ein weiterer Aspekt, welcher die Wichtigkeit der Kategorienbildung definiert, ist die Vergleichbarkeit der Ergebnisse im weiteren Forschungsverlauf. Die festgelegten Kategorien können für die Analyse der folgenden Interviews mitgenutzt werden und werden nur in seltenen Fällen modifiziert.

d) „Gegenstandsbezug statt Technik“ (50)

Die drei Grundverfahren in der qualitativen Inhaltsanalyse sind „Zusammenhang, Explikation und Strukturierung“ (50). Zusammenhang beschreibt den inhaltlichen Kontext, Explikation beschreibt den Inhalt und erklärt diesen und Strukturierung ist charakterisiert durch die sequenzielle Analyse sowie die Kategorienbildung. Wichtig ist hier erneut, dass es sich nicht lediglich um simple Techniken handelt, sondern der Gegenstandsbezug zum Material immer individualistisch ist.

e) "Theoriegeleitetheit der Analyse“ (50-51)

Auch wenn es sich bei der Auswertung der Interviews um praktische Forschungsaktivitäten handelt, sollte der theoretische Hintergrund nie komplett außer Acht gelassen werden. Eine Einbettung in den theoretischen Forschungshintergrund ist bei Forschungsarbeiten unabdingbar. Außerdem ist der Inhalt höher als das Verfahren an sich zu bewerten: „Inhaltliche Argumente sollten in der qualitativen Inhaltsanalyse immer Vorrang vor Verfahrensargumenten haben; Validität geht vor Reliabilität.“ (51). 
f) „Einbezug quantitativer Analyseschritte“ (51)

Auch wenn es sich bei narrativen Interviews um eine qualitative Form der Forschung handelt, sollte bei gewissen Erkenntnissen die quantitative Forschung mit einbezogen werden. Daher ist es durchaus bei gewissen Punkten empfehlenswert, Verallgemeinerungen darzulegen und beispielsweise die Häufigkeit eines bestimmten Verhaltensmusters aufzuzeigen.

g) „Gütekriterien“ (51-52)

Die klassischen Gütekriterien, nämlich Reliabilität, Objektivität und Validität, sind auch in der qualitativen Forschung unabdingbar. Reliabilität und Objektivität sind eng verwandt - beide verlangen eine Nachvollziehbarkeit der Analyse und sind charakterisiert durch die Besonderheit, dass unterschiedliche Forscher oder Forscherinnen beim gleichen Material auf ähnliche Kategoriensysteme bzw. Erkenntnisse stoßen sollten. Die Validität kann nur funktionieren, wenn die anderen beiden Gütekriterien bereits gewährleistet sind. Zum einen fordert die Validität Vergleiche mit anderen Untersuchungsdesigns aus der Literatur und zum anderen werden die gewonnenen Erkenntnisse ebenfalls anhand bestehender Theorien überprüft und verglichen (vgl. Mayring 2010 ${ }^{11}:$ 117).

Doch wie bei jedem theoretischen Konzept gibt es auch kritische Stimmen zur qualitativen Inhaltsanalyse - in den meisten Fällen von Vertretern der hermeneutischen Analyseverfahren, doch teilweise auch von Befürwortern der qualitativen Inhaltsanalyse. So stellen Gläser \& Laudel (20093:199) beispielsweise fest, dass die Inhaltsanalyse Häufigkeiten verwendet und analysiert und hierbei in manchen Fällen das Hervorheben bzw. Extrahieren von Informationen vernachlässigt. Genau aus diesem Grund wird für die folgende Auswertung der Interviews nicht die Reinform der qualitativen Inhaltsanalyse gewählt, sondern zusätzlich der Fokus auf ausgewählte Textstellen in der hermeneutischen Auswertungsmethode gelegt. 
Bevor auf die Nebenanalyseart genauer eingegangen wird, folgt nun ein kurzer Ausschnitt einer beispielhaften Auswertung zur Verdeutlichung der Methodik der qualitativen Inhaltsanalyse.

\subsection{Beispiel der Auswertung mithilfe der qualitativen Inhaltsanalyse}

Bei der folgenden tabellarischen Darstellung handelt es sich um einen Auszug aus dem Interview von Frau Oder von Seite 2 der Transkription:

\begin{tabular}{|c|c|c|c|}
\hline $\begin{array}{l}\text { Zeilen- } \\
\text { nummer }\end{array}$ & Paraphrase & Kategorie & Memo / induktiv \\
\hline $\begin{array}{l}1 / 44 \\
- \\
2 / 10\end{array}$ & $\begin{array}{l}\text { DLRG-Rettungs- } \\
\text { schwimmer } \\
\text { gemacht, doch } \\
\text { musste ihr } \\
\text { Können nie in } \\
\text { brenzliger Situa- } \\
\text { tion unter Beweis } \\
\text { stellen }\end{array}$ & $1 b$ & Rettungsschwimmer \\
\hline $10-17$ & $\begin{array}{l}\text { Zeltlager-Zeit, } \\
\text { viel geschwom- } \\
\text { men, gesungen, } \\
\text { etwas in der } \\
\text { Gruppe unter- } \\
\text { nommen }\end{array}$ & $\begin{array}{l}1 b \\
4 a\end{array}$ & $\begin{array}{l}\text { Zeltlager im Ju- } \\
\text { gendalter }\end{array}$ \\
\hline $17-32$ & $\begin{array}{l}\text { Vater motiviert } \\
\text { nach dem Krieg } \\
\text { zu mehr Bewe- } \\
\text { gung, möchte, }\end{array}$ & $\begin{array}{l}6 a \\
3 a\end{array}$ & $\begin{array}{l}\text { Möchte Vater impo- } \\
\text { nieren, doch klappt } \\
\text { nicht so gut }\end{array}$ \\
\hline
\end{tabular}


Rita Spiller

\begin{tabular}{|c|c|c|c|}
\hline & $\begin{array}{l}\text { dass sie turnt, } \\
\text { doch mäßiger } \\
\text { Erfolg }\end{array}$ & & \\
\hline $35-42$ & $\begin{array}{l}\text { Nach Konfirma- } \\
\text { tion Tanzstunde, } \\
\text { viel Spaß an Be- } \\
\text { wegung zur Mu- } \\
\text { sik, doch } \\
\text { Reihentanz / } \\
\text { Jazztanz reizt sie } \\
\text { mehr als Paar- } \\
\text { tanz }\end{array}$ & $\begin{array}{l}1 \mathrm{~b} \\
3 \mathrm{a}\end{array}$ & $\begin{array}{l}\text { Tanzen im Jugend- } \\
\text { alter }\end{array}$ \\
\hline $\begin{array}{l}42- \\
3 / 9\end{array}$ & $\begin{array}{l}\text { Jazztanz im } \\
\text { Deutschen The- } \\
\text { ater über die } \\
\text { Volkshochschule, } \\
\text { ganz viel Spaß } \\
\text { gemacht }\end{array}$ & $\begin{array}{l}1 \mathrm{~b} \\
3 a\end{array}$ & $\begin{array}{l}\text { Spaß am Jazztanz, } \\
\text { neue Sportart für } \\
\text { sich entdeckt }\end{array}$ \\
\hline
\end{tabular}

Für die Kategorien wurden innerhalb der Analyse folgende Zuweisungen vorgenommen:

1a): Sportart heute

1b): Sportart früher

2a): Häufigkeit Sporttreiben heute

2b): Häufigkeit Sporttreiben früher

3a): Motive pro Sporttreiben

3b): Motive kontra Sporttreiben

4a): Mitsportler/innen

5a): Rolle Gesundheit 
6a): Erfahrungen mit Sport früher

6b): Erfahrungen mit Sport heute

7a): Aktivitäten / Erfahrungen außerhalb des sportlichen Umfelds

Erst am Ende der Analyse werden diese Kategorien wieder zu größeren Hauptkategorien zusammengefügt, aus denen sich anschließend die Kapitel zur Auswertung der Inhalte der Interviews ergeben. Diese Hauptkategorien im Anschluss an die Auswertung sind folgende:

1.) Sportart und Häufigkeit

2.) Motive (inkl. sozialer und Gesundheitsaspekte)

3.) Erfahrungen (mit und ohne Sport $=>$ Schulsport!)

4.) Selbstpräsentation

5.) Sportdefinition

Die Inhalte und Ergebnisse der Interviewauswertungen werden in Kapitel 12 dargelegt und analysiert.

\subsection{Texthermeneutische Auswertungsverfahren}

Nachdem die qualitative Inhaltsanalyse als Hauptauswertungsmethode ausgewählt worden ist, wird an dieser Stelle eine Nebenauswertungsmethode hermeneutischer Art eingeführt. Diese Auswertungsmethode soll bewirken, dass tiefer in das Material hineingelesen wird und mehr Rücksicht auf sprachliche Auffälligkeiten genommen werden kann.

Die so genannte objektive Hermeneutik wurde in den 70er Jahren unter anderen von Ulrich Oevermann entwickelt (vgl. Przyborski \& Wohlrab-Sahr 20103). Der Begriff der Hermeneutik stammt ursprünglich aus dem Griechischen und bedeutet etwa so viel wie „aussagen“ oder "den Sinn einer Aussage 
erklären“ (Kuckartz 2012: 30). Einer der Hauptgründe für die Entwicklung dieses Verfahrens liegt in der Tatsache, dass bis zu dem Zeitpunkt quantitative Analyseformen nicht ausreichend aussagekräftige Ergebnisse produzierten. Grundsätzlich zielt die Objektive Hermeneutik nicht darauf ab, den exakten Inhalt, also das WAS des Gesagten wiederzugeben, sondern konzentriert sich vielmehr auf die Art und Weise des Gesagten, also das WIE (vgl. Helfferich 2005²).

Die Methodologie der Objektiven Hermeneutik befasst sich mit den Unterschieden zwischen subjektiv gemeintem und objektivem Sinn (vgl. hierzu auch Kapitel 2: Methodologie). In der hermeneutischen Analyse sollen stets eine Vielzahl an potenziellen Kontextmöglichkeiten eruiert werden sowie die möglichen (sozialen) Hintergründe einer Äußerung erfasst werden: „Immer geht es also darum, sich der Spezifik des zu erklärenden Sachverhalts dadurch zu nähern, dass man ihn zu anderen möglichen Sachverhalten oder Situationen in Bezug setzt" (Przyborski \& Wohlrab-Sahr 2010: 251).

Wie bei der qualitativen Inhaltsanalyse wird auch hier sequenziell vorgegangen. Es wird also Textstelle nach Textstelle behandelt und analysiert. Zunächst werden sprachliche Merkmale, also etwa semantische Besonderheiten und Charakteristiken sowie auffällige Wortwiederholungen, Unsicherheiten und Gestik und Mimik begutachtet. Diese Art der Analyse fällt bereits unter den Begriff der Feinanalyse (vgl. Przyborski \& Wohlrab-Sahr 20103). Des Weiteren können in einigen Fällen nach Abschluss der sprachlichen Analyse potenzielle Lesarten gebildet werden, welche im Verlauf der Analyse anhand weiterer Textstellen überprüft werden und nach und nach eliminiert werden können. Schließlich bleibt der Kontext, welcher in anderen Fällen auch als vorherrschend erkannt und festgelegt wird.

Auch hier folgt nun ein Beispiel der Auswertung mithilfe des hermeneutischen Verfahrens, welches exemplarisch für die gesamte Analyse der Interviews steht. 


\subsection{Beispiel der Auswertung mithilfe eines hermeneutischen Verfahrens}

Hierbei handelt es sich ebenfalls um einen tabellarischen Ausschnitt aus dem Interview mit Frau Oder von den Seiten 2, 7 und 8 der Transkription:

\begin{tabular}{|c|c|c|c|}
\hline $\begin{array}{l}\text { Zeilen- } \\
\text { nummer }\end{array}$ & Textbeispiel & Sprachlicher Bezug & Memo \\
\hline $\begin{array}{l}2 / 24 \\
-25\end{array}$ & $\begin{array}{l}\text { (Sport } \\
\text { mit dem } \\
\text { Vater } \\
\text { und } \\
\text { zwei } \\
\text { Freun- } \\
\text { dinnen) } \\
\text { "dann } \\
\text { hab ich } \\
\text { mir na- } \\
\text { türlich } \\
\text { welche } \\
\text { gesucht, } \\
\text { die noch } \\
\text { unsport- } \\
\text { licher } \\
\text { waren } \\
\text { als ich } \\
\text { ne [...] } \\
\text { damit } \\
\text { ich gut } \\
\text { ab- } \\
\text { schneid } \\
((\text { lacht))“ }\end{array}$ & $\begin{array}{l}\text { - Dann: Fortsetzung, } \\
\text { Aufzählung } \\
\text { - Ich-Bezug } \\
\text { - Natürlich: als } \\
\text { selbstverständlich } \\
\text { anerkannt } \\
\text { - Welche: unbe- } \\
\text { stimmt, Freundinnen } \\
\text { - Noch unsportlicher: } \\
\text { Steigerung } \\
\text { - Als ich: Vergleich } \\
\text { - Damit: Zweck }\end{array}$ & $\begin{array}{l}\text { Möchte vor ih- } \\
\text { rem sportlich } \\
\text { ambitionierten } \\
\text { Vater nicht als } \\
\text { unsportlich da- } \\
\text { stehen, also } \\
\text { sucht sie sich } \\
\text { Freundinnen, die } \\
\text { noch unsportli- } \\
\text { cher sind: Anse- } \\
\text { hen bei ihrem } \\
\text { Vater ist ihr sehr } \\
\text { wichtig, doch } \\
\text { sportlicher Ehr- } \\
\text { geiz fehlt }\end{array}$ \\
\hline
\end{tabular}




\begin{tabular}{|c|c|c|c|}
\hline $\begin{array}{r}7 / 24 \\
-25\end{array}$ & $\begin{array}{l}\text { (beim } \\
\text { Tanzen } \\
\text { in Ame- } \\
\text { rika) } \\
\text { „ich } \\
\text { wusste } \\
\text { mit mei- } \\
\text { nem } \\
\text { Körper } \\
\text { bis dato } \\
\text { nichts } \\
\text { anzu- } \\
\text { fangen“ }\end{array}$ & $\begin{array}{l}\text { - Ich-Bezug } \\
\text { - Wusste: nicht ange- } \\
\text { eignet } \\
\text { - Körper: Körper- } \\
\text { wahrnehmung } \\
\text { - Bis dato: bis zu be- } \\
\text { stimmtem Zeitpunkt } \\
\text { - Nichts: ohne Aus- } \\
\text { nahme, null }\end{array}$ & $\begin{array}{l}\text { Ihr fehlt jegliches } \\
\text { Körpergefühl, } \\
\text { Körperwahr- } \\
\text { nehmung ist } \\
\text { nicht vorhanden, } \\
\text { hat sich mit ih- } \\
\text { rem Körper nie } \\
\text { vertraut ge- } \\
\text { macht, ist sich } \\
\text { nicht über ihre } \\
\text { eigenen Fähig- } \\
\text { keiten bewusst }\end{array}$ \\
\hline $\begin{array}{l}26- \\
28\end{array}$ & $\begin{array}{l}\text { „[...] zu } \\
\text { merken, } \\
\text { dass da } \\
\text { noch } \\
\text { mehr } \\
\text { als } \\
\text { Arme } \\
\text { und } \\
\text { Beine } \\
\text { sind } \\
\text { sondern } \\
\text { dass da } \\
\text { ja auch } \\
\text { ne Hüfte } \\
\text { ist und } \\
\text { nu-, da-, } \\
\text { da, also } \\
\text { auch } \\
\text { und der } \\
\text { Ober- }\end{array}$ & $\begin{array}{l}\text { - Dass: Begründung, } \\
\text { Kausalzusammen- } \\
\text { hang } \\
\text { - Noch mehr: zusätz- } \\
\text { lich } \\
\text { - Sondern: Gegen- } \\
\text { satz, Gegenargu- } \\
\text { ment } \\
\text { - Ja: selbstverständ- } \\
\text { lich }\end{array}$ & $\begin{array}{l}\text { Lernt dank des } \\
\text { Bewegens zur } \\
\text { Musik eigenen } \\
\text { Körper bewusst } \\
\text { kennen und } \\
\text { entwickelt ein } \\
\text { eigenes Körper- } \\
\text { gefühl }\end{array}$ \\
\hline
\end{tabular}




\begin{tabular}{|l|l|l|l|}
\hline körper & & \\
und & & \\
Schul- & & \\
tern und & & \\
so“ & & \\
\hline
\end{tabular}

Hier ist anschaulich, dass der Fokus viel deutlicher auf einer sehr kleinen Textstelle liegt, diese aber detailliert und tiefergehend ausgewertet wird. In der späteren Auswertung der einzelnen Interviews wird zudem noch auf Gestik und Mimik der Interviewten eingegangen, welche ebenfalls eine wichtige Rolle in der hermeneutischen Auswertung spielen.

\subsection{Kritische Reflexion}

In diesem letzten Kapitelabschnitt vor der eigentlichen Auswertung der Interviews soll im Nachhinein kritisch reflektiert werden, was am eigenen Praxisvorgehen hätte geändert werden können.

Zunächst muss das grundsätzliche Sportverständnis der Interviewten als isoliert betrachtet werden. Hierbei wird deutlich, dass die Interviewten fast alle eine unterschiedliche Vorstellung vom Begriff des Sports zeigen. Nun ist es fraglich, ob eine Klärung dieses Begriffes vorab sinnvoll gewesen wäre. Zum einen wäre das Sportverständnis bei allen Interviewten auf den gleichen Nenner gesetzt worden, zum anderen hätte es dann jedoch nicht die interessanten Vergleiche im Kapitel der Ergebnisse gegeben, wo die unterschiedlichen Sportdefinitionen absichtlich herausgehoben werden. Aufgrund der Ergebnisse im folgenden Kapitel ist dieser Schritt der Vorabklärung also als nicht unbedingt notwendig einzuordnen.

Des Weiteren lässt sich über die grundsätzliche Erhebungsform in Form von qualitativen Interviews diskutieren. Da diese Problematik jedoch bereits zu Beginn des Praxisteils ausführlich dargelegt wurde, wird zu diesem Zeitpunkt darauf nicht näher eingegangen. Es bleibt lediglich festzuhalten, dass in dieser 
Arbeit die Methodik der qualitativen Sozialforschung im Vergleich zur quantitativen Forschung gewählt wurde, um den Grad der Offenheit und den Blick auf das Individuum in möglichst hohem Maße zu ermöglichen.

Die Tatsache, dass in drei der geführten Interviews weitere Menschen neben der Interviewerin und den Befragten anwesend sind, muss ebenfalls kritisch beleuchtet werden. In zwei Fällen, nämlich bei Herrn Schlag, welcher aufgrund seiner körperlichen Einschränkungen auf permanente Begleitung angewiesen ist, und bei Frau Korb, deren Tochter zwar zugegen ist, sich aber nicht in das Gespräch einmischt, ist die Anwesenheit Dritter als unproblematisch und nicht oder kaum beeinflussend einzustufen. Lediglich im Interview mit Herrn Helm liegt möglicherweise eine Beeinflussung seitens der beiden anwesenden Sportkameraden auf die Inhalte und die Verhaltensweise von Herrn Helm vor. Von Verheimlichungen oder falschen Inhalten seiner Erzählungen ist jedoch nicht auszugehen, sondern eher von einer tendenziell bescheideneren Darstellung seiner Person, um sich vor seinen Kumpels nicht zu talentiert und erfolgreich zu präsentieren: „[...] also das ist jetzt nicht übertrieben, eher $\mathrm{n}$ bisschen untertrieben /I:lacht/ (2) aufschnei-, aufschneiden tu ich nicht [Kumpel: Haben wir gar nicht nötig]" (Transkription, S. 3, Z. 15-17). Hier wird deutlich, dass sich die Anwesenden immer wieder durch Kommentare einmischen und so zwar die Atmosphäre deutlich auflockern, jedoch auch für Unterbrechungen im Erzählfluss des Interviewten sorgen. Diese Auffälligkeit sollte bei der Auswertung des Interviews berücksichtigt werden.

Zuletzt ist zum Thema der Rekrutierung der Migrantinnen Stellung zu nehmen. Hier mag die Frage aufkommen, wieso die Befragung der Menschen mit Migrationshintergrund lediglich auf Frauen beschränkt wurde. Die Befragung der Frauen mit Migrationshintergrund erschien besonders reizvoll, weil Frauen aus verschiedenen Ländern gemeinsam befragt werden konnten. Der gesellige Aspekt scheint bei Migrantinnen eher ausgeprägt zu sein als bei ihren männlichen Pendants. Daher wäre es sehr schwierig, vielleicht sogar nicht möglich gewesen, ein Gruppeninterview mit Senioren (insbesondere aus unterschiedlichen Ländern kommend) mit Migrationshintergrund durchzuführen.

Die Überlegung, ein Einzelinterview mit einem Senior mit Migrationshintergrund durchzuführen, wurde ebenfalls verworfen, da bei dieser Art der Befragung 
lediglich ein Mann mit Wurzeln aus einem Staat hätte befragt werden können. Wäre die Zahl der Interviews mit Migranten erhöht worden, um mehrere exemplarische Länderhintergründe vorliegen zu haben, läge der Fokus zu sehr auf den Erzählungen von Menschen mit Migrationshintergrund. Dies war jedoch nicht die Intention dieser Arbeit, weshalb letztendlich lediglich ein Gruppeninterview mit Frauen mit Migrationshintergrund durchgeführt wurde.

Überdies kann das Gruppeninterview als solches kritisch beleuchtet werden. Die leicht entstehende Problematik in einer Gruppendiskussion sind die unterschiedlich großen Redeanteile der Befragten. So kann es vorkommen, dass einige der anwesenden Personen lediglich ein bis zwei Sätze äußern, während andere Interviewte einen deutlich höheren Anteil an Beiträgen haben und ggf. eine Erzählung produzieren. Zusätzlich herrscht ein gewisser Druck innerhalb der Gruppe, sinnvolle Beiträge hinzuzufügen oder gar sehr private Äußerungen abzugeben. Hier besteht die Gefahr, dass die Interviewten ungern intime oder unangenehme Situationen beschreiben, da sie nicht mit der Interviewerin alleine sind. Dennoch wird die Gruppendiskussion für das Interview mit den Migrantinnen als die angemessenste Form der Erhebung angesehen, da die vielen positiven Aspekte, wie das gegenseitige Unterstützen, sowohl sprachlich als auch inhaltlich, deutlich überwiegen.

Weitere kritische Beleuchtungen bezüglich der Inhalte folgen nach der Analyse der Interviews im Zuge der Diskussion.

\section{Ergebnisse der Interviewauswertungen}

Im folgenden Kapitel werden die konkreten Ergebnisse der Interviewauswertungen präsentiert. Als nachvollziehbare Darstellung wird eine Aufteilung der Ergebnisse nach Kategorien innerhalb der qualitativen Inhaltsanalyse gewählt. Die Hauptkategorien bilden jeweils die Überschriften der Kapitel, wohingegen die Subkategorien Unterkapitel betiteln. Eine Darstellung aller Einzelfälle wird aufgrund der großen Stichprobe von insgesamt 20 Interviews 
verworfen, da hier keine allgemeine Aussage, sondern zum einen lediglich Einzelfallanalysen hätten erhoben werden können sowie zum anderen eine deutlich zu umfangreiche Fokussierung auf das Individuum stattgefunden hätte.

\subsection{Definition des Sportbegriffs}

Wie bereits im Theorieteil (siehe Kapitel 3.2) angeschnitten, ist die Definition von Sport an sich ein sehr kontrovers diskutiertes Thema, welches in der Literatur für unterschiedliche Ergebnisse sorgt (vgl. z.B. Röthig \& Prohl 2003). Für die vorliegende empirische Untersuchung wird Sport weitestgehend mit regelmäßiger Bewegung gleichgesetzt, wobei das regelmäßige Ausführen von Hunden oder das tägliche Fahrradfahren zum Einkaufen nicht unter diesen Sportbegriff fallen, weil das Hauptaugenmerk dabei nicht auf der Bewegung liegt. Sport ist also im Folgenden stets assoziiert mit entweder der aktiven Teilnahme an Kursen in Vereinen, Fitnessstudios oder individuellem Sporttreiben, wie etwa Walken, Radwandern oder Schwimmen.

Da jedoch der Sportbegriff mit den Interviewpartnerinnen und -partnern vor Beginn des Interviews nicht näher definiert wurde, ist es im Nachhinein von Bedeutung, die individuelle Sportdefinition der Interviewten zu analysieren, da jede Erzählung über Sporttreiben ein individuelles Sportverständnis beinhaltet.

Oftmals wird in den Interviews der Sportbegriff gar nicht hinterfragt bzw. die persönliche Sportdefinition als selbstverständlich angesehen und nicht angezweifelt. Dies zeigt sich beispielsweise im Interview von Frau Oder (die Namen der Interviewpartner/innen entsprechen im Folgenden nicht den eigentlichen Namen, wie bereits in Kapitel 10.6 erläutert), welche bereits das Herunterrutschen der Treppe mittels Pappkartons als erstes Sporttreiben klassifiziert (vgl. Seite 1, Z. 12-14). Zudem bezeichnet sie das Gebären ihrer Zwillinge als sportliche Aktivität: „[...] und dann kam der erste Sport, dass das Kind, das erste Kind geboren wurde und dann kam der zweite Sport, das zweite Kind musste dann geholt werden mit Kaiserschnitt [...]" (Seite 8, Z. 40-42). Im weiteren Verlauf des Interviews distanziert sie sich zunehmend von dem so genannten klassischen Sportverständnis, indem sie ihre Neigung zur 
Waldorfpädagogik kundtut, wo „alles, was mit dem Fuß getreten wird, [...] kein Sport [war]“ (Seite 10, Z. 46-47). Herr Helm legt in seiner Erzählung hingegen den Schwerpunkt auf das Fußballspielen in seiner Jugend, da dies für ihn die Hauptfreizeitbeschäftigung gewesen war, bevor er in das Berufsleben einstieg (vgl. Seite 1, Z. 7 ff.).

Während die Aktivität Fahrradfahren von manchen Interviewten selbstverständlich als Sportart definiert wird, wie z.B. von Herrn Schlag („Der Spbeste Sport ist der Radsport (Seite 16, Z.34)) oder von Herrn Meister („[...] Radfahren ist Sport [...]" (Seite 6, Z. 17)), ist das Rad in anderen Fällen lediglich ein Fortbewegungsmittel oder das Radfahren eine nicht ambitionierte Freizeitaktivität. Herr Taum fährt beispielsweise viel Fahrrad, behauptet jedoch, dass er „nicht in dem Sinn Sport getrieben hat“ (Seite 19, Z. 23-24) und Frau Klaus nutzt das Fahrrad lediglich als Mittel zum Zweck: „[...] und Fahrrad [fahren] aber auch nur hier wirklich äh, also auch nicht sportlich sondern einfach nur bewegungstechnisch [...] na, ökologisch oder, wie auch immer ((lacht)) [...]" (Seite 3, Z. 11-13). Herr Schulz ist sich hingegen zunächst nicht sicher, ob Radfahren als Sport klassifiziert werden kann, bejaht dies nach kurzer Überlegung schließlich aber: „Das Radfahren früher [...] (6) also das, das was, wenn man das Sport nennen kann aber doch, das war Sport, ja (2) aber das habe ich nicht so, verinnerlicht, ne /l:mhm/ (3) dass das Sport war (2) [...]" (Seite 7, Z. 6-8). Hier wird auch deutlich, dass eine Definition von Sport gar nicht als selbstverständlich vorausgesetzt werden kann, das heißt, nicht jeder Interviewpartner oder jede Interviewpartnerin ist sich dessen bewusst, was Sport eigentlich für inn oder sie selbst bedeutet.

Die Unsicherheit bezüglich der Definition des Sportbegriffs zeigt sich teilweise auch während des Interviews, wenn die Befragten den Sportbegriff zum Thema machen. So ergibt sich beispielsweise folgende Situation im Interview mit Frau Oder, welche vom abendlichen Tanzen erzählt: „[...] ist aber auch irgendwie ne Art von Sport oder wie:, würden, würden Sie sagen, ist das einfach nur Vergnügen [...]“ (Seite 16, Z. 29-31). Hier wird ihr erst im Laufe ihrer Erzählung bewusst, dass sie möglicherweise von der Thematik des Sporttreibens abschweift und vergewissert sich daher mithilfe einer Nachfrage an die 
Interviewerin, ob dies der Fall sei. Die direkte Interaktion mit der Interviewerin findet ebenfalls im Interview mit Frau Schmal statt, welche über das Walken spricht: „[...] vielleicht finden Sie das keinen Sport, aber ich finde das $\mathrm{n}$ Sport [...]" (Seite 3, Z. 39-40). Ein weiteres Beispiel, welches die Unsicherheit der Begriffsnutzung zeigt, ist eine Passage aus dem Interview mit Frau Klum, die über das Motorradfahren spricht: „[...] ich weiß nicht, es kann man ja nicht als sportliche Betätigung, sagen, aber es geht so'n bisschen in die Richtung äh, äh, zumindest sich bewegen [...]" (Seite 2, Z. 10-12).

Überdies sprechen die Interviewten oftmals von Ersatzbetätigungen, die sie dem Sporttreiben entweder gleichsetzen oder mit inm zumindest ansatzweise vergleichen. Im Interview spricht Herr Fahne über seine Arbeit als Jugendlicher auf einem Bauernhof, welche er als extrem belastend und körperlich fordernd empfand, weshalb er die Tätigkeit durchaus als Sport ansieht (vgl. Seite 13). Auch Herr Meister vergleicht Gartenarbeit mit sportlicher Betätigung, setzt diese jedoch in Anführungszeichen, da er den Begriff Sport nicht per se mit Gartenarbeit gleichsetzen möchte (vgl. Seite 1). Herr Schulz ist ebenfalls der Meinung, dass körperliche Arbeit im Beruf für ausreichend Bewegung sorgt und weitere sportliche Aktivitäten somit überflüssig sind (vgl. Seite 2).

Im Interview mit den Migrantinnen wird deutlich, dass der Sportbegriff nicht hinterfragt wird bzw. der Sportbegriff individuell als gegeben hingenommen wird. Bei deutlichen Abweichungen allerdings wird dieser thematisiert: Eine Befragte (Frau Popow) empfindet die Betreuung ihrer Mutter als Sport: „[...] mit der Oma hab ich's zu tun (1) das ist auch Sport (2) aufstehen, hinlegen, umziehen [...]" (Seite 2, Z. 44-45). Hier rechtfertigt sie das Pflegen ihrer Mutter als dem Sport gleichzusetzende Ersatzbetätigung und entschuldigt gleichzeitig ihren Mangel an Zeit für andere sportliche Betätigung aufgrund der intensiven Betreuung ihrer Mutter.

Nachdem die Schwierigkeit bzw. die Problematik des einheitlichen Verständnisses des Sportbegriffes aufgezeigt und analysiert wurde, folgen nun im nächsten Kapitel eine Übersicht und ein Vergleich der tatsächlich ausgeführten unterschiedlichen Sportarten. 


\subsection{Sportarten}

Im Folgenden werden die Unterschiede zwischen den ausgeübten Sportarten in der Kindheit und Jugend (Sportarten früher) mit den aktuell getriebenen Sportarten im Seniorenalter (Sportarten heute) aufgezeigt und verglichen.

\subsubsection{Sportarten früher}

Die Interviewten, welche über ihre Kindheit erzählen, erwähnen beispielsweise das Spielen in der Nachbarschaft mit anderen Kindern als erste sportliche Betätigung (vgl. u.a. Herrn Schramm, Frau Limes, Frau Hilke und Frau Korb). Nach einer kurzen Kritik am Sportunterricht, in dem viel zu wenig Spezifisches geübt wurde, bezieht sich Frau Korb auf genau diese Thematik: „[...] aber auf der anderen Seite haben wir sehr viel (1) Sport (2) ähm (2) auf b- auf der Straße, so nachmittags gemacht, ne, also sehr viel Federball gespielt, äh, meine Mutter die hat immer so=mit=so=m Brummkreisel da $s$-sind wir immer so durchs ganze Dorf [...] diese kleinen Kreisel (1) /l:mhm/ und dann muss man das mit, mit der Peitsche immer so [...]" (Seite 2, Z. 16-20). Hierbei wird insbesondere durch die Verwendung des Subjektpronomens "wir" deutlich, dass Sport in den frühen Jugendjahren bei dieser Gruppe von Interviewten sehr sozial orientiert war und keinen Leistungszwang kannte.

Der Wettkampfgedanke ist deutlicher vertreten bei denjenigen, die als Kinder und Jugendliche in Vereinen organisiert waren. Hier wurden sowohl Sportarten wie Schwimmen und Leichtathletik, welche eher dem Individualsport zuzuordnen sind, als auch Sportarten wie Handball, Basketball und Fußball genannt, welche zu den klassischen Mannschaftssportarten gehören. Auffällig ist, dass keine( $r$ ) der Interviewten ihre/seine spezifische Sportart aus dem Jugendalter im höheren Alter fortführt. Selbst die früher sehr leistungsorientierten Vereinssportler, wie Herr Klaus und Herr Helm, die mit ihren Mannschaften in einer überregionalen Auswahl spielten, geben ihre Sportarten mit Beginn des Erwachsenenalters bzw. mit Eintritt in das 
Berufsleben auf. Die Gründe hierfür werden im Kapitel zum Thema der Motive (vgl. Kapitel 11.3) aufgezeigt.

Bei den Migrantinnen zeigt sich grundsätzlich ein vergleichsweise ähnliches Bild, nämlich dass in der Kindheit noch relativ regelmäßig Sport getrieben wurde, dieses dann mit Eintritt in das Erwachsenenalter abebbte und erst im Seniorenalter in manchen Fällen wieder aufgenommen wurde. Die Sportarten, welche als Kinder betrieben wurden, unterscheiden sich allein schon aus geographischen Gründen von den Sportarten, welche die Seniorinnen und Senioren ohne Migrationshintergrund trieben. Die Migrantinnen betätigten sich vornehmlich in Wintersportarten wie Skifahren oder Schlittschuhlaufen (Frau Smirnow, Frau Popow, Frau Koslow). Frau Sokolow hebt sich aufgrund der genannten Sportarten (Rudern, Volleyball und Fechten) deutlich von dem Rest der Gruppe ab. Hiermit hat sie einen anderen Bezug zum Sport und teilt die Erfahrungen der anderen mit Wintersport nicht. Wie sich diese Impressionen auf das heutige Sportverhalten übertragen, wird im nächsten Unterkapitel aufgezeigt.

Jene, die in ihrer Kindheit gar keinen Sport trieben, mussten oftmals andere körperliche Tätigkeiten verrichten. So wuchsen insbesondere die Landkinder mit vielen landwirtschaftlichen Aufgaben auf und wurden innerhalb der Familie immer für derartige Aufgaben benötigt (vgl. u.a. Herrn Fahne, Herrn Meister und Frau Limes). Herr Fahne beschreibt seinen Alltag nach Feierabend während der Lehre beispielsweise folgendermaßen: „[...] tja, dann hatten wir noch ne kleine Landwirtschaft, ja denn (1) jeden Abend losgehen, Grünfutter für die Ziegen holen, oder jetzt auch (1) andere, Feldarbeiten mitmachen, es ist nicht jeden Tag, aber immer wieder oder, dann mal bei nem, Bauern mithelfen, nech, der für uns das Land (1) zurecht gemacht hat, dem musste man dann helfen, joa waren viele Abende, und viele Tage waren dann eben halt (1) dass man voll beschäftigt war (2) da hab ich also, keine Sehnsucht nach Sport gehabt [...]" (Seite 1, Z. 25-31).

Eine noch prägendere Vergangenheit schildert Herr Schramm, der als ältester Befragter noch in den Kriegsjahren aufwuchs und während des Krieges um das Überleben kämpfen musste. Selbst direkt nach dem Ende des Krieges war der Alltag durch den ständigen Kampf gegen das Verhungern charakterisiert: „[...] 
und da hatten wir dann (1) Trockenverpflegung, Kekse und Schmalz, das war die Verpflegung die wir hatten für drei Wochen /l:oh Gott/ musste das reichen, ne /l:ja/ und dann kriegte ich Sodbrennen, sage ich Ihnen ((lacht)) wär ich bald verrückt geworden /I:oh/ vor allen Dingen nachts, ne /I:ja/ da musste ich im Wald nachts aufhalten, damit die Polen uns nicht erwischen, ne, die hätten uns ja sofort gemeldet, das sind (1) aber wir hatten gleich vorsichtshalber unsere Dinger abgeschnitten, unsere Hosen und haben denn, KLV, Kinderlandverschickung, weil so, wir sahen ja aus wie Kinder noch, nech, 16, jetzt war ich inzwischen 17 geworden, ne (1) und das war, war nicht dolle (1) na und dann kam ich nach Göttingen (2) und wie ich dann zurückkam (1) hatten, an Sport und so was hatten wir gar nicht gedacht=wir hatten nur Hunger, Hunger und mussten Arbeit kriegen [...]“ (Seite 3, Z. 34-45). Dass in diesen Zeiten und unter diesen Umständen nicht an sportliche Betätigung gedacht wurde, ist kaum verwunderlich.

\subsubsection{Sportarten heute}

Die aktuell betriebenen Sportarten der Interviewten wurden bereits in der Vorstellung der Stichprobe in Kapitel 8.3 des empirischen Teils graphisch dargestellt, wobei es sich hier um eine visuelle und quantitative Darstellung handelt, die in diesem Kapitel unter qualitativen Aspekten genauer analysiert werden soll.

Zunächst wird die Gruppe der Nicht-Sportler betrachtet, welche einerseits geprägt ist durch so genannte Ersatzbetätigungen, die sportlicher Betätigung aus ihrer Sicht nahekommen oder andererseits durch unregelmäßiges und nicht konsequentes Sporttreiben. So beschreibt Frau Oder zum Beispiel ihre große Passion für Jazztanz, geht dieser Leidenschaft jedoch nicht nach, sondern verliert sich zunehmend in damaligen Träumen ihrer Jugend (vgl. u.a. Seite 16) oder schmiedet vage Zukunftspläne: „[...] dann werde ich also im [Verein] w-, werde ich mehr machen, da gibt's internationale Tänze /I:mhm/ da möchte ich mal hingehen, das werde ich mal machen [...]" (Seite 16, Z. 5-7). Hier wird insbesondere durch die Verwendung von ,mal' deutlich, dass diese Aussage 
kein fester Entschluss, sondern ein eher lose formuliertes Vorhaben ist, welches jedoch nicht unbedingt erfüllt werden wird.

Frau Klaus beschreibt Renovierungsarbeiten am Haus als ausgleichende Tätigkeiten und ihr Mann schließt sich dieser Sichtweise unabhängig von ihr an. Hinzu kommt bei inm die körperliche Komponente im Berufsleben, da er als Arzt immer mit dem Heben, Stützen oder Tragen von Patienten konfrontiert wird. Als weitere Ersatzbetätigung deklariert Frau Limes inre Gartenarbeit, da es aufgrund des großen Grundstückes immer Arbeit gibt. So kommt sie zu dem Schluss, dass Rasenmähen durchaus mit Nordic Walking gleichzusetzen ist (vgl. Seite $7 \mathrm{ff}$.).

Frau Zack beschreibt keine so genannten alternativen ausgleichenden Tätigkeiten, stellt sich jedoch besonders dank ihrer Gestik und Mimik als sehr lebhafter und agiler Typ dar, welcher daher, laut eigener Aussage, allein durch Kommunikation und soziale Interaktion sehr viel Bewegung hat.

Bei der Gruppe der Sporttreibenden ist die Häufigkeit der regelmäßigen sportlichen Aktivitäten der unterscheidende Faktor. Während Herr Fahne höchstens zweimal die Woche schwimmt und auch Herr Meier einmal zur Aquagymnastik und einmal zur Hockergymnastik geht, betreibt Frau Schmidt tägliches Walken und wöchentliche Aquagymnastik. Diese präsentiert sich als leidenschaftliche Seniorensportlerin, welche als Kind unsportlich war und keine Sportart für sich finden konnte. Bei inr ist der Schritt vom unsportlichen Kind über ein Erwachsenenalter ohne sportliche Aktivitäten bis hin zur aktiven Seniorensportlerin mit Abstand der größte.

Bei den Migrantinnen treibt heute nur eine sehr geringe Anzahl Sport. Hervorzuheben ist Frau Sokolow, welche in der Kindheit sehr aktiv Sport trieb und durch die früher betriebenen, eher ausgefallenen Sportarten wie Rudern oder Fechten aufgefallen ist (siehe voriges Kapitel). Sie betätigt sich heutzutage regelmäßig im Fitnessstudio und ist der Überzeugung, dass ihr Training die vorherrschenden Rückenprobleme lindert und sie ohne das Training deutlich mehr Schmerzen ausgesetzt wäre. 
Diese Intention der Schmerzlinderung lässt sich auch auf die anderen Fitnessstudio-Besucher übertragen. Während Frau Beier den Besuch im Fitnessstudio eher als Termin, den es zu erledigen gilt, sieht, bilden sich in der Trainingsgruppe um Herrn Helm gewissermaßen Rituale, die neben dem dreimaligen Trainieren zur Schmerzlinderung stets das Beisammensitzen in einer eingeschworenen Gruppe von drei Trainingspartnern einschließt. Diese vertraute Runde ist charakterisiert durch humorvolle, anstachelnde, flapsige Gespräche, welche jedoch stets in eine herzliche Atmosphäre gebettet sind. So wird während des Interviews mit Herrn Helm, bei dem auch seine beiden Trainingspartner anwesend sind, erwähnt, welch Ehre es doch für die Interviewerin sei, mit am Tisch sitzen zu dürfen: „[Kumpel: Ich mein du hast äh, man muss wirklich sagen äh, bist ne, besondere Ausnahme, dass du hier an dem Tisch sitzen darfst] /I: Ich fühle mich geehrt/ Normalerweise, nee, darf keiner hier sitzen, nee, nee [...]" (Seite 6, Z. 29-31). Hier wird deutlich, wie stark die Verbindung zwischen den drei Trainierenden mittlerweile gewachsen ist und wie sehr sie sich in ihrer Gruppe wohlfühlen. Der Sport ist also hier zum Teil auch Mittel zur Verständigung und Mittel zur Geselligkeit. Genau um diese Thematik soll es im nächsten Kapitel gehen.

\subsection{Motive}

In diesem Kapitel geht es um folgende Fragestellungen: Welche übergeordneten Konzepte bzw. Motive überzeugen Seniorinnen und Senioren vom Sporttreiben? Welche Motive halten die Seniorinnen und Senioren vom Sporttreiben $a b$ ?

\subsubsection{Motive pro Sporttreiben}

Ein Motiv, welches für viele Interviewte eine wichtige Rolle beim Thema Sporttreiben spielt, ist der gesundheitliche Aspekt. Bei den Interviewten Frau Korb, Herrn Schlag, Herrn Schulz, Frau Beier, Frau Klum und Frau Schmal 
prägen lebensverändernde Schicksalsschläge wie Herzinfarkte, Schlaganfälle, Bandscheibenvorfälle, Krebserkrankungen, Hirnblutungen oder allgemeine gesundheitliche Zusammenbrüche den Eintritt in das Sportlerleben. Die Gemeinsamkeiten dieser Schicksale führen alle zu dem eiligen Beginn des aktiven Sporttreibens, oftmals auf Anraten der Ärzte. Die plötzlich auftretenden gesundheitlichen Probleme fungieren in diesen Fällen als eine Art ,Warnschuss' oder Signal für eine dringende Veränderung im Alltag, welche durch Sporttreiben umgesetzt wird.

Frau Koch tritt beispielsweise auf Anraten ihres Arztes in eine Herzsportgruppe ein, hat so den schweren Start (Anfangsschwierigkeiten, negative Erlebnisse wegen Kurzatmigkeit etc.) überwunden und ist nun im Verein in mehreren Funktionsgymnastikgruppen tätig. Außerdem betreibt sie im Anschluss an zwei Hüft-Operationen ebenfalls auf Anraten ihres Arztes Wassergymnastik. Frau Koch ist ein markantes Beispiel für eine Nicht-Sportlerin, die nach einem gesundheitlichen Schicksalsschlag nun eine ehrgeizige Vereinssportlerin geworden ist, welche ihren Sport nicht mehr missen möchte: „[...] und, wie gesagt, es macht, wahnsinnig Spaß [...]" (Seite 3, Z. 4-5).

Ein ähnliches Beispiel kristallisiert sich im Interview mit Frau Klum heraus: Diese sagt von sich aus, dass ihr sportliches Leben erst im Jahr 2000 begonnen hat, ebenfalls mit einem gesundheitlichen Schicksalsschlag, welcher völlig unerwartet eintrifft. Der ersten gesundheitlichen Einschränkung erfolgt bereits ein halbes Jahr später die nächste und spätestens nachdem sie daraufhin an Krebs erkrankt ist, wird ihr bewusst, dass sie ihr Leben ändern muss. Aufgrund der deutlichen Signale, welche ihr Körper aussendet, realisiert sie, dass sie ihrem Körper mehr Bewegung ermöglichen muss, um überhaupt die Folgen der Erkrankung kompensieren zu können. Sie schafft es dank ihrer Disziplin, ihre körperlichen Einschränkungen weitestgehend zu eliminieren und lebt in ihrem neu gewonnenen ,Sportlerleben" wieder auf. Gleichzeitig gibt sie jedoch auch offen zu, dass ihr Leben ohne ihre Krankheiten einen eher nichtsportlichen Verlauf genommen hätte: „[...] bei mir wär's auch so [unsportlich] geworden, denke ich, wenn ich nicht krank geworden wäre [...]“" (Seite 2, Z. 3334). Während sie in ihren Individualsportarten wie Schwimmen oder Walken ihre neu gewonnenen sportlichen Erfahrungen genießt, tut sie sich mit der 
Eingliederung in die Gymnastikgruppe im Verein (noch) ein wenig schwer: „[...] wenn ich da in die Umkleide komme ((lacht)) dann würd ich, könnte ich ja schon langsam verrückt werden, das ist so laut und so ein Gequackel, dädädädädädädä, also immer, ich denke immer, wie so ne, so ne Gänsegruppe /l:lacht/ die so, so (1) ganz laut schnattert, ne, und das ist mir manchmal zu viel, dann, ziehe ich mich um und gin- gehe in einen anderen Raum, weil ich das überhaupt nicht aushalten kann [...]" (Seite 9, Z. 14-19). Innerhalb dieser Aussage wird deutlich, dass ihr das Sporttreiben aus gesundheitlicher Sicht sehr gut tut, sie sich aber nicht mit der Vereinsgruppe identifizieren kann bzw. möchte und daher Sport nicht aus geselligen Gründen treibt. Sie betont zudem, dass sie sich nicht als „Vereinsmensch“ sieht (Seite 9, Z. 29).

Frau Schmidt ist ebenfalls kein Vereinsmensch, sondern liebt es, alleine für sich zu walken oder Rad zu fahren. Früher war sie immer die Außenseiterin als einziges unsportliches Familienmitglied in ihrem sehr sportlichen Elternhaus. Sie beschreibt ihre Kindheit dennoch nicht grundsätzlich als negativ, sondern sie hatte auch schöne Erlebnisse „bis auf dieses ungute Gefühl, dass ich, n Kuckucksei war in der Familie, dass ich nicht so gut war ((lacht)) wie, wie die anderen [...]" (Seite 16, Z. 3-4).

Die Unsportlichkeit bzw. ihre Einstellung zum Sporttreiben ändert sich bei Frau Schmidt schlagartig nach einem gesundheitlichen Schicksalsschlag, welcher ihr Leben komplett verändert. Sie realisiert nun, dass ihr Leben ohne sportliche Aktivität nicht wieder bergauf gehen würde und ist fest entschlossen, regelmäßig Sport zu treiben. Dieser unbändige Wille spiegelt sich in ihren sportlichen Tätigkeiten wider, nämlich in ihrer Disziplin, jeden Tag sportlich aktiv zu sein - sei es beim Walken, beim Radfahren oder bei der Wassergymnastik. Nach und nach steigert sich Frau Schmidt in eine Abhängigkeit vom Sporttreiben, welche zunächst wegen ihrer Willensstärke als sehr positiv erscheint („,...] das, das ist mir richtig zum, zum zweiten Leben geworden, dieses Walken [...]" Seite 3, Z. 38-39).), am Ende jedoch Fragen aufwirft bezüglich ihrer krampfhaften, fast zwanghaften Not, Sport treiben zu müssen: „[...] das ist auch $n$ Zwang (1) das muss ich einfach schaffen [...] ich bin eigentlich ne Getriebene (1) ich bin getrieben von dem Gedanken, ich werde steif (1) ich kann gar nichts mehr /l:mhm/ das, da, das, das ist richtig, das ist 
fast schon krankhaft, aber wenn ich es, ich weiß nicht wie ich da rauskommen kann, aber ich möchte es auch nicht missen, wenn, wenn ich es getan habe, bin ich glücklich ((lacht)) [...]" (Seite 4, Z. 16-23). Hier klingt es so, als sei Frau Schmidt zwar einerseits besessen von ihrem Ehrgeiz und Drang, Sport zu treiben, doch andererseits genießt sie diesen Zwang auch, weil er sie glücklich macht. Obwohl der Verdacht aufkommt, ihr Zwang stelle aus ihrer Sicht etwas Negatives dar, versichert sie bei einer spezifischen Nachfrage im weiteren Verlauf des Interviews, dass dieser Zwang deutlich positiv sei: „Nein, es ist positiv [...] es ist auch nicht, ich empfinde es nicht als krankhaft [...] äh, ich empfinde es einfach als, Notwendigkeit, als, als, als, wie, wie man, wie man etwas tun muss um etwas Schönes zu erreichen [...]" (Seite 12, Z. 15-17). Hier erscheint zum ersten Mal eine gewisse Ambivalenz innerhalb der Aussagen von Frau Schmidt. Während sie zuvor von ihrem Drang, Sport zu treiben als nahezu krankhaft berichtet, versichert sie hier nun explizit, dass dieser Zwang keinesfalls krankhaft sei.

Nichtsdestotrotz sind ihre Motive ursprünglich charakterisiert durch gesundheitliche Aspekte, vornehmlich geprägt durch die plötzliche Veränderung im Leben aufgrund einer Krankheit.

Bei Herrn Schlag ist dieses Motiv auch vorherrschend, wobei sein Ehrgeiz bezüglich des regelmäßigen Sporttreibens deutlich unter dem von Frau Schmidt anzusiedeln ist. Er betont vermehrt, dass er lediglich Sport treibt, wenn er Zeit und Lust dazu hat. Als er große gesundheitliche Probleme bekommt, ist auch inm schnell klar, dass er nicht weiterleben können wird wie bisher. So lernt er während seiner Aufenthalte in zahlreichen Rehakliniken bereits kennen, wie wichtig regelmäßige sportliche Betätigung für seinen gesundheitlichen Aufschwung ist. Daher trainiert er auch heute noch an Geräten im Fitnessstudio, geht im Normalfall (bei Zeit und Lust) einmal wöchentlich zur Gymnastik und unternimmt regelmäßige Fahrradausflüge mit seiner Frau. Dass seine Priorität auf dem Fahrradfahren bzw. Radwandern liegt, wird im Verlauf des Interviews schnell deutlich. „[...] so, und jetzt ist=es=so=wenn=wa Zeit haben und Lust haben vor allen Dingen, gehen wir zwei Mal die Woche hierher [Fitnessstudio], einmal die Woche, äh, zum Sportverein, Gymnastik, und ansonsten, bei jeder Gelegenheit (1) Fahrradfahren [...]" (Seite 2, Z. 37-40). 
Zudem gibt er selbst zu, dass er gern auf das Training an Fitnessgeräten und die Gymnastik verzichten würde, wenn es ihm körperlich besser ginge: „[...] und hätt=ich die gerun- sundheitlichen Einschränkungen nicht, säße ich sicherlich in der Sofaecke und würde goa nüscht /l:((lacht))/ außer Radfahren (1) denn das ist=was=was=was ja schön ist=was einem gefällt, ne [...]" (Seite 2-3, Z. 50-2). Hier wird erneut deutlich, wie stark die Motivation zur sportlichen Betätigung durch (gesundheitliche) Beeinträchtigungen geprägt sein kann.

Doch auch andere Motive spielen bei dem Anreiz zur sportlichen Betätigung eine Rolle. Frau Nickel treibt heutzutage beispielsweise aus geselligen und sozialen Gründen Sport und empfindet viel Freude bei der Teilnahme an wöchentlichen Gymnastikgruppen. Als Beleg für diese These fungiert folgende Textstelle bei der Beantwortung der Frage nach dem schönsten Sporterlebnis: „[...] es sind alles schöne Erlebnisse (1) nech (1) dass=wa uns jetzt zur Weihnachtsfeier treffen, ich weiß nicht ob das n ((lacht)) /l:ja/ joa, das ist auch, da kann man sich mal privat so'n bisschen beschnuppern, ne (1) also da so zu Weihnachtsessen so, ne, das (1) find ich schon okay /l:mhm/ dass man sich mal außerhalb (1) trifft, nech=so hat man ja nicht viel Zeit (1) nech, sich zu unterhalten aber ich denke das ist immer so'n richtig Schönes, schöne Sache einmal im Jahr [...]" (Seite 5, Z. 28-34). Zusätzlich formuliert Frau Nickel den ursprünglichen Wunsch, sich zum Beginn der Rente ein Hobby suchen zu wollen, damit ihr Alltag nicht plötzlich von einer abrupten Leere gefüllt ist. Erst dann erwähnt sie, dass auch der Arzt ihr zu sportlicher Aktivität rät, damit sie ihr Gewicht ein wenig reduzieren kann, was sie mit Erfolg umsetzt.

Auch Herr Meister macht sich bereits vor dem Eintritt in das Rentenalter Gedanken über die Alltagsgestaltung nach der Pension. Er nimmt sich vor, Krankheiten bewusst vorzubeugen und Sport ausschließlich aus präventiven Gesichtspunkten zu treiben. Im Vergleich zu Frau Nickel erweckt Herr Meister jedoch den Eindruck, als wäre dieser nicht mit Freude bei der Sache, da er in keiner Weise etwas wie einen Spaßfaktor erwähnt. Für inn ist der Sport eine Art Pflichttermin, der wöchentlich auf inn wartet und einen Ausgleich zum Alltag darstellen soll. Auch wenn inm laut eigener Aussage nicht viel an der 
Gruppengemeinschaft in seinem Verein liegt, lobt er später jedoch trotzdem die Gemeinschaft und den Zusammenhalt unter den Sporttreibenden (vgl. Seite 6).

Geselligkeit ist ebenfalls bei Herrn Helm das aktuell vorherrschende Hauptmotiv zum Sporttreiben im Fitnessstudio. Mit seinen beiden Mitsportlern, welche er vor fünf Jahren vor Ort kennenlernte, hat sich mittlerweile eine unzertrennliche Freundschaft gebildet, die jedes Training zu einem positiven Pflichttermin verwandelt. Die Bedeutung des gemeinsamen Trainings innerhalb dieser Gruppe wird unter anderem in dieser Aussage Herrn Helms deutlich: „[...] das kommt auch dazu, wenn man (1) wenn ich jetzt allein wäre, äh, wäre ich, hätte ich [Kumpel: Würde man liegen bleiben] das eine oder andere Mal auch gesagt, och, heute, nö, ich hab keine große Lust, ich bleib zu Hause, aber dadurch dass man so zu dritt ist und äh, da sagt der, sagt ja jeder praktisch dann, ach nee (1) Kumpel kommt wieder [Kumpel: Die warten] die warten, also steht man auf, und es gibt also nur ganz geringe, das müssten schon wirklich wichtige Termine sein, Arzttermin oder, oder das äh so, irgend, andere schwerwiegende Sachen, dann äh (1) dann äh, gehen wir nicht /I:mhm/ aber sonst verlegen wir die (1) normalen Termine so, dass wir unseren Sport also, immer machen können [...]“ (Seite 5, Z. 8-18).

Dieses Hauptmotiv hat sich jedoch erst im Laufe seines aktiven Sportlerlebens herauskristallisiert. Ursprünglich beginnt er sein Training, um einer Bandscheiben-Operation vorzubeugen. Hier wird deutlich, dass Motive oftmals entweder fließend ineinander übergehen, d.h. sich im Laufe der Zeit wandeln können, oder zeitgleich mehrere Motive vorherrschen können, welche die Seniorinnen und Senioren zur sportlichen Betätigung antreiben.

\subsubsection{Motive kontra Sporttreiben}

Nachdem im vorigen Kapitel die Motive aufgezeigt wurden, die zum Sporttreiben führen, soll hier der Fokus auf die Motive gelegt werden, welche Seniorinnen und Senioren vom Sporttreiben abhalten. 
Zunächst ist die bemerkenswerte Verwandlung Frau Nickels zu erwähnen, welche bereits im vorigen Kapitel durch ihren Sporteifer und Elan charakterisiert wurde. Hier soll nun aufgezeigt werden, dass dieser Ehrgeiz und Spaß nicht ihre grundsätzliche bzw. ursprüngliche Einstellung dem Sport gegenüber darstellte. Frau Nickel wurde durch mangelnde bzw. falsche Vorstellung, was die Sportart Gymnastik angeht, stets vom Sporttreiben abgehalten. Sie assoziierte Gymnastik mit Bundeswehr-Drill und war daher immer abgeschreckt: „[...] naja: hab ich gedacht Gymnastik machste auf keinen Fall /l:lacht/ das ist ja wie bei der Bundeswehr, rauf runter, nee, und Kniebeuge so was (1) machst du nicht [...]" (Seite 1, Z. 24-26). Zusätzlich zu dieser falschen Sportvorstellung kamen Unsicherheiten und Bedenken inklusive einer gewissen Angst vor der Blamage in einer unbekannten Gruppe. Auf die Frage, wie diese Bedenken aussahen, antwortet Frau Nickel Folgendes: „Naja:, dass=se mich auslachen (1) weil ich dann zu steif bin, nech, und die sagen gucke mal die da=mei:ne Güte, die bewegt sich ja so ne, wie ne lahme Ente=nee hab ich gedacht sowas (1) irgendwie hab ich Hemmungen gehabt [...]" (Seite 4, Z. 41 43). Hier wird deutlich, dass solche als schwerwiegend einzustufenden Probleme im Prozess der Sportannäherung zunächst überwunden werden müssen.

Ein häufig auftretendes Motiv gegen das Sporttreiben ist das Ausüben anderer, meist im musikalischen Bereich einzuordnender Hobbys. Herr Taum und Frau Oder singen beispielsweise beide im Chor und spielen Musikinstrumente. Ihnen mangelt es daher nicht an Geselligkeit, da Musikvereine ebenfalls ein soziales Umfeld gewährleisten und Aktivitäten über das wöchentliche Treffen hinaus anbieten.

Außerdem beschreiben die interviewten Nicht-Sportler viele so genannte Ersatzbetätigungen als ihre Kompensation von Alltagsarbeit, wie zum Beispiel Gartenarbeit, Renovierungsarbeiten am Haus oder bauliche Tätigkeiten. Lediglich Frau Zack hat ein eher ungewöhnliches Kontra-Argument gegen das Sporttreiben. Sie möchte aufgrund ihrer bereits sehr schlanken Figur nicht noch mehr Gewicht verlieren: „[...] alles andere war für mich deshalb nie relevant, weil ich mehr meine (1) äh, Pfunde die ich habe irgendwie äh, äh (1) dadurch noch, loszuwerden, also das war eigentlich nicht so, mein, und dann auch, die 
gewisse Trägheit und Faulheit mich da, aufzuraffen [...] hatte ich niemals den Biss dazu (1) bin ich ehrlich (1)" (Seite 1, Z. 20-26). Hier wird deutlich, dass sie zudem nie große Lust hatte, sich zum Sport zu motivieren und Sport nie für notwendig hielt. Diese Einstellung hat sich bis heute nicht geändert. Auch sie ist in einer geselligen Gruppe im Verein tätig, welche ihrer Meinung nach „ganz viel Zeit in Anspruch [nimmt]" (Seite 2, Z. 21), ein Argument, welches sie mehrmals betont (vgl. auch Z. 15, Z. 19 und Z.25). Hinzu kommt ihr sehr agiler Lebensstil inklusive einer stark ausgeprägten Mimik und Gestik, welche in gewissen Zügen an sportliche Bewegung erinnert.

Auch Frau Yildiz, eine der interviewten Migrantinnen, entschuldigt ihr mangelndes Sportinteresse aufgrund ihrer konstant gebliebenen schlanken Figur, wegen der sie, laut eigener Aussage, Sport „[...] nicht nötig“ habe (Seite 2, Z. 21).

Nichtsdestotrotz ist ein aktiver Lebensstil nicht mit regelmäßiger sportlicher Betätigung gleichzusetzen - er hilft lediglich, den Kreislauf anzuregen, verglichen mit Seniorinnen und Senioren, welche theoretisch den Großteil des Tages sitzend auf dem Sofa verbringen und einen eher bewegungslosen Lebensstil verkörpern.

\subsection{Schulsport (Erfahrungen / Erlebnisse)}

Der Schulsport ist für die meisten Interviewten die erste Erfahrung mit dem regelmäßigen Sporttreiben und für manche paradoxerweise ebenfalls die letzte Erfahrung. Inwieweit Schulsport Auswirkungen auf die spätere Sportkarriere bzw. die Motivation zum regelmäßigen Sporttreiben hat und wie Schulsport eine vielleicht zuvor positive Sporteinstellung durch Negativerfahrungen trüben kann, wird in diesem Kapitel dargestellt..

Zunächst sollen die positiven Schulsporterfahrungen aufgezeigt werden. Am euphorischsten berichtet Herr Klaus von seiner Schulzeit. Sowohl der Lehrer als auch die Inhalte des Sportunterrichts sind in positiver Erinnerung geblieben, insbesondere die freiwillig durchgeführte Leistungs-AG im Basketball, dank der 
er überregional für eine Auswahl der Stadt nominiert wurde. Zusätzlich zur Basketball-AG trainierte er mit seinen Mannschaftskollegen Langstreckenausdauer, um konditionell noch fitter im Spiel sein zu können. Seine Basketball-Euphorie wurde lediglich durch seine Eltern gedämpft, denen ein guter Schulabschluss wichtiger war als eine sportliche Karriere des Sohnes. Heutzutage unterstützt und vertritt Herr Klaus einen ähnlichen Standpunkt und ist froh über das harte Durchgreifen und die Verbote seiner Eltern. Dies spiegelt sich auch im Verlauf seines Lebens wider: Obwohl er in der Jugend prägende Erfahrungen mit dem Sporttreiben machte, konzentriert er sich im Erwachsenenalter auf seinen Beruf und sieht andere Dinge als wichtiger an als Sport. So ist er heute zwar nicht ohne Bewegung, treibt aber im klassischen Sinne keinen Sport, sondern verbraucht seine Energie beim Renovieren seiner Häuser oder bei seiner täglichen Arbeit als Arzt. Innerhalb dieses Berufes sieht er nun auch die gefährlichen Aspekte des Sports, wenn er Verletzungen behandeln muss und körperliche Degeneration im Leistungssport beobachtet. Dennoch ist er der Meinung, dass körperliche Betätigung unabdingbar ist für ein gesundes und ausgeglichenes Leben.

Die Erzählungen anderer Interviewten bezüglich des Sportunterrichts fallen deutlich weniger positiv aus. Oftmals erinnern sich die Interviewten gar nicht richtig an den Sportunterricht und spekulieren über ihre Erfahrungen und Gefühle von damals. Als wenig auffällig und ereignisreich beschreiben sowohl Frau Korb, Frau Zack, Frau Klum als auch Herr Fahne sowie Herr Schlag ihren Sportunterricht.

Ihre Erinnerungen sind vage und das Thema Schulsport nimmt nur sehr kurze Teile der Haupterzählungen im Interview ein. Grund für diese eher bedeutungslose Einstellung zum Schulsport ist vermutlich die eigene sportliche Leistung, welche bei diesen Interviewten als relativ durchschnittlich beschrieben und eingeschätzt wird. Herr Schlag ist zudem der Meinung, dass der Schulsport keine prägende Wirkung auf inn hatte - weder im positiven noch im negativen Sinn.

Bei Frau Klum ist es ein wenig anders. Sie hatte bereits im Schulkind-Alter eine deutliche Abneigung gegen Mannschaftssportarten, welche sich bis ins heutige Seniorenalter fortsetzt. Sie möchte sich nie mit anderen vergleichen oder 
messen: „[...] diese ganzen Sportarten, ähm, die m-mit vielen Leuten, so, so Mannschaftssportarten, hab ich gehasst /l:lacht/ wie die Pest, Volleyball zum Beispiel, furchtbar /l:lacht/ ((lacht)) und das ist bis heute so, ich würde niemals irgendwie ne Sportart machen können, äh, wo ich äh, mich mit anderen äh, unmittelbar, in Konkurrenz befinde oder, oder äh, wo ich äh, so'n Gruppengefühl entwickeln muss /l:mhm/ weil das grundsätzlich überhaupt nicht so mein Ding ist (1) [...]" (Seite 1-2, Z. 49-3). Diese Einstellung hat bis heute nicht an Aktualität verloren - auch heute ist sie im Verein nicht auf soziale Kontakte aus, sondern treibt Sport nur für sich selbst und ihrer Gesundheit zuliebe.

Eine Ausnahme bildet Frau Nickel, welche keine Erfahrungen aus dem Sportunterricht zu berichten hat, da sie nie Sportunterricht hatte. Dies stellt sich für ihre spätere Vorstellung von sportlicher Betätigung als problematisch heraus, als sie beispielsweise im Erwachsenenalter nicht weiß, was Gymnastik eigentlich ist und daher Hemmungen entwickelt, sportliche Betätigung in einer Gruppe zu betreiben. Ihre mangelnde Erfahrung bzw. ihr Unwissen hemmt sie zunächst, aktiv Sport zu betreiben.

In der Erzählung von Frau Klaus zeigt sich, wie der Sportunterricht negative Einflüsse auf das Sportverhalten der Kinder und Jugendlichen hervorrufen kann. Der Sportunterricht ist ihr in furchtbarer Erinnerung geblieben, da er durch die nationalsozialistische Zeit geprägt war und stets den Schwerpunkt auf Drill und Körperertüchtigung legte (,halt diese BDM-Scheiße" [Bund Deutscher Mädel] (Seite 2, Z. 26)). Für sie ist Sport immer eher mit Kampf und Druck verbunden, sodass sie froh ist, nach der Schule keinen Sport mehr treiben zu müssen. Auch wenn Sport hier ein einseitiges Bild zeigt, hat es sie derart geprägt, dass für sie sportliche Betätigung nie wieder in Frage gekommen ist.

Bei Herrn Schlag war der Sportunterricht ebenfalls auf Körperertüchtigung ausgelegt aufgrund des bevorstehenden Krieges. Er erinnert sich wenig an Inhalte des Schulsports, da er wegen seines Rundrückens oftmals am orthopädischen Turnen teilnehmen musste und aufgrund dieser Rückenproblematik nicht immer den normalen Sportunterricht erlebte. 
Auch Frau Liebig hatte bereits während der Schulzeit eine körperliche Einschränkung, welche jedoch erst diagnostiziert wird, als sie 55 Jahre alt ist: Ihr räumliches Sehen funktioniert bis heute nicht einwandfrei, weshalb sie Entfernungen nicht einschätzen kann und daher im Schulsport nie in der Lage war, einen Ball zu fangen. Hinzu kam ihre schlechte Wurftechnik, sodass Fangen und Werfen im Schulsport stets mit extremen Negativerfahrungen assoziiert werden. Außerdem fehlten ihr Erfolgserlebnisse bei den Bundesjugendspielen, sodass sie frustriert war und nicht genug Ehrgeiz entwickelte, diese Leistungen zu verbessern. Auch hier haben die Erfahrungen im Schulsport Einfluss auf ihr späteres Leben - sie bleibt sportlich inaktiv und entwickelt zwar Wünsche, Sportarten oder sportliche Aktivitäten auszuprobieren, setzt diese jedoch nie in die Tat um.

Die Erzählung von Herrn Taum bezüglich seines früheren Sportunterrichts ist ebenfalls geprägt von Negativerfahrungen. Insbesondere im Winter war der Sportunterricht charakterisiert durch Volkstanz und Turnen. In beiden Disziplinen gab Herr Taum wortwörtlich eine schlechte Figur ab, da er aufgrund seiner langen und dürren Körperstatur Probleme hatte, seinen Körper zu koordinieren. Dies spiegelte sich dementsprechend in seiner Note im Winterhalbjahr wider: Er kam nie über eine Fünf hinaus und war daher das eine oder andere Mal versetzungsgefährdet. Als erschwerend kam laut Meinung des Interviewten der Sportlehrer hinzu, welcher inn nach einer gewissen Zeit in eine Schublade steckte und nicht erkannte, dass er stets bemüht war, seine Note aufzubessern. Alles in allem ist der Schulsport und damit auch der Sport allgemein negativ konnotiert - vermutlich auch ein Grund, warum Herr Taum eher die musikalische Richtung einschlägt und in der Musik ein Hobby findet, welches ihm das Bilden eines sozialen Netzwerks ermöglicht.

Frau Schmal, Herr Schulz und Herr Meister repräsentieren die Gruppe der Interviewten, die extreme Negativerfahrungen im Schulsport erlebten und heute im Seniorenalter trotzdem Sport treiben. Herr Meister beschreibt den Schulsport nach dem Krieg ebenfalls als noch stark militärisch angehaucht, geprägt von Disziplin und Drill. Der Schulsport umfasste beispielsweise Aufgaben, wie barfuß durch den Schnee laufen als so genannte Abhärtungsmaßnahme. Der Sportunterricht setzte hier sehr schlechte Voraussetzungen für eine sportliche 
Zukunft, u.a. mangels der Vermittlung von unterschiedlichen Sportarten sowie wegen einer überstrengen Lehrmethode. Es lässt sich vermuten, dass diese Sportabneigung während des Erwachsenenalters vorherrschend ist und Herr Meister selbst heute von der Sportausübung nicht völlig überzeugt ist. Er erkennt zwar die Notwendigkeit des Sporttreibens, ist jedoch kein passionierter Vereinssportler und hält sich auch in der Häufigkeit des Sporttreibens pro Woche zurück, damit Sport keinesfalls in „Hetzerei“ (Seite 4, Z. 42) ausartet.

Herr Schulz ist in diesem Punkt noch ein wenig extremer, da sowohl die Schulsporterfahrungen negativer ausfallen als auch die heutige Lust zum Sporttreiben sehr eingeschränkt vorhanden ist. In seiner Erzählung über den Schulsport zeigt Herr Schulz auf, wie unsinnig und überflüssig Schulsport für inn in seiner Kindheit und Jugend erschien. Zum einen konnte er Turnübungen nie korrekt ausführen und war daher bei seinen Klassenkameraden nicht sehr beliebt, insbesondere wenn es um das Aufstellen von Mannschaften ging, wobei er stets zuletzt gewählt wurde. Zum anderen wurde er auch von den Lehrern bestraft, beispielsweise durch Ohrfeigen, wenn er Übungen nicht korrekt ausführte. Wie stark seine Abneigung gegenüber dem Schulsport ist, zeigt sich in folgender Aussage: „[...] ich war, von Anfang an als Kind, eine Niete (1) ich war eine Sportniete, wenn es darum ging da mal so'n äh, (1) Purzelbaum, beziehungsweise ne Hechtrolle hieß das früher (1) über mehrere Personen zu machen, da bin ich auf'm Bauch gefallen oder irgendwas=ich konnte nie, meinen Körper so abrollen, wie das eigentlich gedacht war, und (1) erntete natürlich auch dadurch so'n bisschen den Spott der, anderen (1) in der Vornazizeit kann man sagen, äh (1) Schüler die dann doch, äh, alle gedrillt wurden, so'n bisschen auf äh, Körperertüchtigung hieß das damals und auf äh (1) na so'n bisschen vormilitärisch auch, Zeit so ne /l:mhm/ (1) joa, das ging dann, weiter (1) ich hatte also nie eine, ein Hang zum Sport oder auch eine, ein=ein, Verlangen nach Sport [...]" (Seite 1, Z. 7-17).

Hier zeigt sich bereits sprachlich, inwiefern sich Herr Schulz von dem Rest seiner Klasse abgrenzt. Mit der Formulierung „die anderen“ präsentiert er sich auch semantisch als Außenseiter und Einzelgänger. Auch heute gehört Sporttreiben nicht zu einer seiner Lieblingsbeschäftigungen. Im Grunde genommen ist Sport für inn ein absolutes Mittel zum Zweck, nämlich die 
Erhaltung der Bewegungsfunktion seiner Wirbelsäule und das Reduzieren seiner Schmerzen auf ein aushaltbares Maß. Das heißt, dass Sport inm heute genauso wenig Spaß und Freude bereitet wie damals, doch heute erkennt er die Wichtigkeit von Bewegung, insbesondere in seinem Alter.

Im Fall von Frau Schmal entwickelt sich ein etwas anderes Bild. Ihre Schulsporterlebnisse sind inhaltlich durchaus mit denen von Herrn Schulz zu vergleichen. Auch sie hat sehr prägende negative Schulsporterinnerungen, da sie nie gute Leistungen erbrachte und bei den Bundesjugendspielen nie eine Urkunde gewinnen konnte. Hinzu kam ihr psychisches Problem, welches sie mit dem Darstellen ihres Körpers im Laufe der Kindheit entwickelte; sie fühlte sich zu dick und schämte sich dementsprechend, ihren Körper vor den Klassenkameraden (insbesondere beim Schwimmen im Badeanzug) zu präsentieren. „[...] ich war, ich hieß auch Pummelchen [...] ich war, äh wohl immer $\mathrm{n}$ bisschen (1) ja, nicht phlegmatisch, aber $\mathrm{n}$ bisschen dick /l:mhm/ würde ich sagen (1) also habe ich in der Pubertä:t (1) in der Schule, auch, sehr ungern Sport gemacht (1) weil ich irgendwie immer, meinen Körper nicht zur Schau stellen wollte [...]" (Seite 1, Z. 12-15). Dieses Unwohlsein wurde zudem seitens ihrer Familie verstärkt, da sie nie Lob oder Zuspruch bezüglich ihrer sportlichen Leistungen erfuhr und somit kein Selbstbewusstsein im Hinblick auf sportliche Aktivitäten entwickeln konnte.

Umso unerwarteter ist ihr heutiger Wille, regelmäßig Sport zu treiben, der allerdings erst durch eine Erkrankung geweckt wurde und ihr Bewusstsein für Sport so schärfte, dass sie inzwischen sportliche Aktivitäten in den Alltag integriert hat. Es lässt sich vermuten, dass ihr Wille und Anreiz bezüglich sportlicher Aktivität ohne diese gesundheitliche Einschränkung nicht geweckt worden wäre und sie weiterhin inaktiv leben würde. Somit hat der Sportunterricht auch hier eine zunächst sehr negative Wirkung auf den anschließenden Lebensabschnitt, nämlich das frühe Erwachsenenalter, in dem sie sportlich inaktiv lebte und aufgrund der Negativerlebnisse keine sportliche Aktivität zu vermissen schien.

Um noch etwas tiefer auf die Psyche sowie die charakteristischen Eigenschaften der Befragten eingehen zu können, wird im folgenden Kapitel die Selbstpräsentation der Interviewten beschrieben und analysiert. Hierbei ist die 
Differenz der eigenen Beschreibung zu dem eigentlichen Tun von besonderer Bedeutung.

\subsection{Selbstpräsentation}

Bei den Migrantinnen ist die Selbstpräsentation alleinig anhand der Länge der Redeanteile im Verlauf der Interviews zu charakterisieren. Während einige Frauen zwar bei dem Gruppeninterview anwesend sind, jedoch nichts sagen, weil sie entweder nicht in die Zielgruppe passen oder ihre Deutschkenntnisse zu schwach sind, erzählen andere verhältnismäßig viel über ihre sportliche Vergangenheit und Gegenwart.

Frau Smirnow ist beispielsweise sehr offen: Sie scheut sich nicht, ihren Namen preiszugeben und interessiert sich nicht für die Ansprache über die Anonymisierung der persönlichen Daten. Sie ist selbstbewusst, bringt sich eigenständig ins Gespräch ein und versucht, eine Vertrauensbasis mit der Interviewerin aufzubauen, indem sie Gegenfragen stellt. Dass sie hierbei die strengen ungeschriebenen Regeln des Interviews verletzt, ist ihr nicht bewusst - sie handelt vielmehr aus Interesse am Gegenüber.

Auch Frau Popow bringt sich vermehrt in das Interview ein und fungiert ein bisschen als die nicht offiziell festgelegte Dolmetscherin. Sie übersetzt die Erzählaufforderung ins Russische (die Sprache, die von den meisten Migrantinnen entweder gesprochen oder zumindest teilweise verstanden wird) und hinterfragt das gegenseitige Verständnis auf beiden Seiten. In einer Situation wird aufgrund ihrer Formulierung deutlich, dass sie für die Gruppe spricht: „[...] aber wir haben alle Probleme mit der Wirbelsäule [...]“ (Seite 3, Z. 19). Hier ist erkennbar, dass sie aufgrund der Sprache in der Lage ist, für andere zu sprechen, ohne deren konkrete Krankheitsgeschichte zu kennen. Dies ist jedoch nicht als überheblich zu werten, sondern eher als nett gemeinte Hilfe für ihre Bekannten. Ihre hilfsbereite und selbstbewusste Art bringt sie in den Vordergrund, obwohl ihre Deutschkenntnisse grammatikalisch betrachtet nicht herausragend sind. 
Obendrein als aktiv zu beschreiben ist Frau Sokolow. Sie ist die Initiatorin des ersten Redebeitrages und bringt sich auch anschließend immer wieder produktiv und informativ in den Gesprächsfluss ein. Dies gibt auch den anderen Interviewten einen Anstoß zu neuen Gedankengängen und Erzählungen.

Im Großen und Ganzen bleibt festzuhalten, dass die umfangreicheren Redebeiträge von den Migrantinnen stammen, die der deutschen Sprache mächtiger sind als andere. Nichtsdestotrotz bekommen alle in die Zielgruppe passende Migrantinnen dank der gegenseitigen sprachlichen Übersetzungshilfe die Möglichkeit, ihre Erfahrungen kundzutun.

In den anderen Interviews kann die Kategorie ,Selbstpräsentation' grob in drei Gruppen geteilt werden: Zum einen existiert eine kleine Gruppe von eher wortkargen und distanzierten Interviewten, welche einerseits nicht viel zu erzählen hat, andererseits den Anschein erweckt, als würde sie nicht so viel erzählen wollen bzw. ihr Privatleben möglichst wenig preisgeben wollen. Hierbei handelt es sich beispielsweise um Frau Zack, Frau Korb und Frau Hilke, welche zudem durch ihre Gestik und Mimik Distanz signalisieren, indem sie selten den direkten Blickkontakt suchen, sondern eher seitlich in die Ferne schauen.

Diese drei Interviews sind gekennzeichnet durch viele sprachliche Auffälligkeiten, wie z.B. ,äh', ,öh' etc., welche auf die eigene Unsicherheit schließen lassen. Hinzu kommen in einigen Passagen Hinweise auf eine gewisse Verschlossenheit, die sich beispielsweise durch folgende Aussagen äußert: „[...] da kann ich jetzt eigentlich so: wollte ich mich da nicht drüber [...]“ (Interview mit Frau Hilke, Seite 2, Z. 40) oder „[...] ja, das war's jetzt aber wirklich“" (Interview mit Frau Korb, Seite 1 (!), Z. 41).

Obwohl Frau Korb heutzutage regelmäßig Gymnastik im Verein betreibt, grenzt sie sich sprachlich von den so genannten ,Sportlern“ ab: „Ja, das sind Sportler, die sind also (3) offen" (Seite 4, Z. 11-12). Hier wird deutlich, dass sie die Offenheit der Sportler, welche sie selbst nicht besitzt, beneidet. Sie erkennt richtig, dass inr diese Offenheit fehlt. Deshalb bezeichnet sie sich selbst nicht als vollkommene Sportlerin. Trotzdem ist ihr Leben gekennzeichnet durch eine große Entwicklung in Bezug auf das Sporttreiben, welches heute bei ihr als regelmäßige Freizeitbeschäftigung klassifiziert werden kann. Ihre Unsicherheit 
wird überdies untermalt durch die Anwesenheit ihrer Tochter und Nichte, welche sich während des Interviews im gleichen Raum befinden. Vielleicht fühlt sich Frau Korb im vertrauten Umfeld mit ihrer Familie wohler und sorgt demnach dafür, dass ihre Tochter beim Interview neben ihr sitzt. Diese scheint jedoch im Vorhinein nichts von dem Interviewtermin gewusst zu haben, was ihrem verwunderten Gesichtsausdruck und der Nachfrage, was nun passieren solle, zu entnehmen ist.

Zum anderen lässt sich eine Gruppe aus sehr selbstbewussten Seniorinnen und Senioren bilden, welche unabhängig von ihrer aktuellen Sportaktivität offen und selbstbewusst auftreten. Sie präsentieren sich als lebensbejahend und erfreuen sich ihres Alltags - ob mit oder ohne Sport.

Allen voran ist Frau Oder zu nennen, welche sowohl im Interview als auch in ihrem sonstigen Privatleben laut eigener Aussage kein Blatt vor den Mund nimmt und stets sagt, was sie denkt. Aufgrund ihrer direkten Art stößt sie bei anderen Personen oft auf Unverständnis bzw. zum Teil sogar auf Ablehnung. Wie wichtig inr ein lebensbejahendes Verhalten ist, zeigt sich in ihrem Entschluss, aus einem betreuten Wohnen ,zurück' in eine eigene Wohnung zu ziehen. Ihre Begründung für diesen Schritt drückt sie mit klaren Worten aus: „[...] ich will le:ben (1) L-E-B-E-N [...]" (Seite 25, Z. 11). Diesen aussagekräftigen, laut ausgesprochenen Ausruf bekräftigt sie mithilfe einer starken Gestik, bei der sie die Hand hebt und jeden Buchstaben imaginär in die Luft malt.

Ebenfalls lebensbejahend und als Optimist präsentiert sich Herr Schlag. Obwohl oder vielleicht gerade weil er durch seine Krankheitsgeschichte sehr starke Rückschläge einstecken musste, schätzt er seinen aktuellen Gesundheitszustand, der inm zumindest theoretisch erlaubt, regelmäßig Sport zu treiben. Trotz diverser schlimmer Diagnosen seitens der Ärzte inklusive „achtzig chronische[r] Krankheiten“ (Seite 1, Z. 27) definiert er diese eher als "gesundheitliche Einschränkungen" (Seite 2, Z. 50-51) und verkörpert somit eine salutogenetische Sichtweise in Bezug auf die Thematik Gesundheit vs. Krankheit (vgl. Kapitel 2.3 im Theorieteil). Nichtsdestotrotz ist er sich selbst gegenüber ehrlich und zählt sich eindeutig nicht zu den Vollblutsportlern, welche von Natur aus sportliche Veranlagungen mitbringen: „,...] und hätt=ich 
die gerun-gesundheitlichen Einschränkungen nicht, säße ich sicherlich in der Sofaecke und würde goa nüscht [...]" (Seite 2, Z. 50-51). Hierbei winkt er ab und beendet den unvollständigen Satz mit einem Augenzwinkern.

Herr und Frau Klaus stellen sich beide als sehr kumpelhaft und locker dar. Ähnlich locker ist ihre Sichtweise, was das Thema Sporttreiben angeht, da sie beide keiner regelmäßigen sportlichen Tätigkeit nachgehen und diese auch nicht zu vermissen scheinen. Frau Klaus stellt sich zusätzlich als extrem jugendlich dar und versucht vielleicht ihre Unsportlichkeit mit verbaler Verjüngung wettzumachen. Sie assoziiert die Sportlermentalität mit Offenheit und Lockerheit und möchte dank ihrer Ausdrucksweise ebenso wirken, obwohl sie streng genommen nicht der Gruppe der Sportler zuzuordnen ist. Beispiele für ihre umgangssprachliche Ausdrucksweise sind etwa Wörter wie "Titten zwicken“ (Seite 1, Z. 21), „war schon heavy“ (Seite 2, Z. 33), "Sockenschuss“ (Seite 2, Z.41) oder "meinen Ollen“ (Seite 3, Z.5). Hinzu kommt ihre auffällig präsente Gestik, indem sie oftmals Handbewegungen macht, welche Gleichgültigkeit oder Lässigkeit symbolisieren. Auch ihre Mimik ist teilweise geprägt von ungewöhnlichen Gesichtszügen bzw. einer Art Schmolllippe, indem sie ihre Unterlippe vorschiebt und erneut betont locker aufzutreten scheint.

Die Gemeinsamkeiten zwischen Herrn Helm und Herrn Fahne sind auf den ersten Blick nicht so leicht auszumachen. Während Herr Helm deutlich jünger ist und der gesellige Aspekt des Sporttreibens an erster Stelle steht, erscheint Herr Fahne körperlich bereits etwas weniger agil im Vergleich zu Herrn Helm. Auch seine Motive haben einen anderen Hintergrund, da er sich als Individualsportler stark auf sich selbst konzentriert und eine Gruppe um sich herum als störend empfindet. Dennoch verfolgen beide Senioren ein gleiches Ziel: Sie wollen, so lange wie ihr Körper es innen erlaubt, regelmäßig Sport treiben.

Angetrieben werden beide durch die guten gesundheitlichen Resultate, die durch das Sporttreiben erzielt werden. Herr Fahne bekommt bei Arztbesuchen regelmäßig Komplimente bezüglich seines (für sein Alter) überdurchschnittlichen Fitnesszustandes. Auch beim freiwilligen Verteilen von Post zeigt er sich ehrgeizig, wenn Nachbarn inn auf seinem Fahrrad beobachten: „[...] vor, drei vier Jahren (1) hatte ich auch mal n Brief zuzustellen 
und äh, geht's n (1) ganz kurzes Stück aber auch sehr steil da rauf ne (1) und da standen unten an der Kreuzung ein paar Bekannte /l:schmunzelt/ na, hab ich angehalten, habe $\mathrm{n}$ Moment gesprochen, na wo willst du denn hin, ich sage ich will da oben n Brief hinbringen (1) was, denn willst da, äh, gehste aber zu Fuß rauf, ich sag nee, da fahre ich rauf, das gibt's nicht, da kann man nicht mit dem Fahrrad rauffahren, ich sag soll ich's dir zeigen /l:lacht/ ja wenn man n Fahrrad mit Gangschaltung hat dann geht das denn (1) gibt man sich keine Blöße und denn fährt man da rauf [...]" (Seite 4-5, Z. 44-1). Diese kleinen, aber sehr essentiellen Erfolgserlebnisse treiben inn immer wieder an, sich nicht auf dem Sofa niederzulassen und die Beine hochzulegen, sondern stets an seine Gesundheit zu denken und diese mit ausreichend Bewegung zu erhalten. Unterstützt werden diese Passagen darüber hinaus durch ein leichtes Lächeln, welches die momentane Präsenz der Erinnerung darstellt und inn gedanklich und emotional in die damalige Gefühlslage zurückbringt.

Herr Helm verdankt seiner sportlichen Disziplin die Vermeidung einer Operation am Rücken. Auch hier ist die primäre Intention gesundheitlicher Natur. Doch heute beschreibt er unter anderem sein schönstes Sporterlebnis als die Tatsache, dass er seine zwei Trainingskollegen kennenlernen durfte und nun „praktisch, davon ausgeht, dass wir solange zum Sport gehen (1) äh, wie es uns äh, sage ich mal, unsere Zeit, oder unser Leben erlaubt [...]" (Seite 6, Z. 23). In dieser Aussage wird insbesondere durch die ,wir-Form' erneut die starke Bindung der drei Freunde deutlich, deren Gemeinschaft und Willensstärke einen ganz großen Teil zur sportlichen Aktivität beitragen. Auch während des Interviews kommuniziert Herr Helm durch häufigen Blickkontakt mit seinen beiden Trainingspartnern, welche mit am Tisch sitzen und ebenfalls Kommentare abgeben. Diese Aussage bezüglich der Trainingseuphorie ist dennoch mit Vorsicht zu genießen, da Herr Helm durch die Anwesenheit seiner Trainingspartner ggf. indirekt zu einer positiven Aussage bezüglich der Freundschaft gedrängt wird (vgl. Kapitel 10.3).

Letztendlich existiert die dritte Gruppe der Seniorinnen und Senioren, welche sich eher als Opfer ihrer persönlichen, meist gesundheitlichen Verfassung präsentiert. 
Frau Limes stellt sich selbst als sehr offen und erzählfreudig dar. Trotz der ungewohnten Interviewsituation entsteht schnell eine hohe Vertrauensbasis, in der sie viele persönliche und private Dinge preisgibt. Ihr Verhalten in gewissen Lebenssituationen ist oftmals ambivalent: Während sie zum einen spontan und häufig eine deutliche Begeisterung oder Faszination für gewisse Sportgeräte oder sportliche Aktivitäten entwickelt, die sie auch emotional fröhlich präsentiert, ist sie andererseits in vielen Fällen nicht willensstark genug, um Ideen und Wünsche in die Tat umzusetzen. Im Zuge dieses Aufgebens kommen Selbstzweifel an die Oberfläche und ihr fehlt der letzte eigene Impuls, um Worte in Taten umzusetzen. Zudem lässt sie sich oft von den Meinungen anderer beeinflussen und akzeptiert z.B. die Hypothese, dass gewisse sportliche Aktivitäten vielleicht eher für jüngere Menschen vorgesehen sind, obwohl ihre eigene Tochter diese Meinung nicht äußert. Konkret ausgeführt werden im Interview zwei Situationen, welche ihre charakterliche Schwäche diesbezüglich darlegen. Die erste Situation handelt von ihrem Wunsch, das Inlineskaten auszuprobieren: „[...] dann hab ich mal gesagt, ich will noch mal Inliner fahren (3) hab mir auch welche gekauft (1) mit Knie- und Ellenbogenschützer, und (1) hab gedacht, ach das ist toll und hab dann meine jüngste Tochter, die lebt in Berlin, die hab ich dann gefragt, öh, üben wir mal zusammen und dann hat die gesagt, ja, als erstes müssen wir mal bremsen üben und so /l:lacht/ und dann hab ich gedacht, also bist du bescheuert, du bist jetzt, 59 Jahre alt, jetzt willst du anfangen, äh, Inliner zu fahren [...] bei dem ersten Sturz den du machst und wenn du dann nen Oberschenkenhalsbruch hast ((lacht)) dann sagen die Leute, wie kann man denn so bescheuert sein [...]" (Seite 5, Z. 32-40). Parallel zu dieser Textpassage klopft sie sich mit ihrem Finger an die Stirn und schüttelt den Kopf.

Hier wird besonders deutlich, welche Vorurteile sie sich zu eigen gemacht hat und welche Wirkung diese potenziellen Meinungen auf ihre Verhaltensentscheidung haben. Eine weitere Situation, welche inhaltlich ähnliche Muster aufweist, handelt von einem Sommerurlaub auf Mallorca, in dem Frau Limes gerne das Reiten ausprobieren möchte: „[...] ich hab immer gedacht, oh, ich möchte so gerne jetzt noch mal, ich möchte so gerne mal reiten, also ich möchte jetzt mal ohne Angst /l:mhm/ auf'm Pferd sitzen und möchte das mal erleben und dann waren wir mal, ähm, auf Mallorca (1) mit äh, nem 
Arbeitskollegen und seiner Frau, also, könnten eigentlich meine Kinder sein, so, junges Paar, mein Mann und ich, wir vier, und da gab's das Angebot, dass man mit äh, dem Pferd quasi so am Strand reiten kann, da hab ich gesagt, das mach ich, und dann haben die gesagt, u:nd, dann hast du, weil ich gesagt habe, ich hab in diesem Urlaub das erste Mal nur Sachen eingepackt, die ich wirklich brauche, eine, Hose [...] und, dann haben sie gesagt, du hast nur eine Wanderhose mit und eine für abends äh, zum Essen gehen, ne, so (lacht) und wenn du jetzt diesen Ritt machst, dann stinkst du die restlichen Tage nach Pferd /l:lacht/ (1) hab ich's nicht gemacht /I:oh/ (3) [...]" (Seite 12, Z. 7-20). In der kurz entstandenen Pause nach dieser Geschichte blickt sie wehmütig in die Ferne - die Enttäuschung über die ausgelassene Möglichkeit lässt sich ebenfalls in ihrer Mimik ablesen.

Diese Situationen lassen vermuten, dass sie als nicht sehr durchsetzungsfähig und eigenständig zu charakterisieren ist. Dies stimmt jedoch in anderen Situationen nicht. Nach dem Tod ihres Mannes, von welchem sie sehr emotional und manchmal auch unter Tränen erzählt, lernt sie zu akzeptieren, dass sie nicht alles selbst können muss und auch Hilfe von anderen Menschen in Anspruch nehmen darf und so über ihren eigenen Schatten zu springen lernt. Zudem sitzt sie auf Feiern nicht alleine traurig in einer Ecke, sondern fragt hin und wieder die Männer von Freundinnen, ob sie mit ihr tanzen möchten. Hier zeigt sie sich als sehr selbstbewusst und willensstark.

Ein ambivalentes Verhaltensmuster, sogar in zweierlei Hinsicht, ist auch bei Frau Schmal zu erkennen. Zum einen spricht sie sich deutlich für den Individualsport aus und gegen den Gruppensport. Sie behauptet mehrfach, am liebsten ganz alleine für sich Sport zu treiben, um sportliche Etappen für sich selbst meistern zu können, weil sie sich in der Gruppe nicht wohl fühlt: „Ich mach alles alleine /l:ja, ja/ auch das, das, das, das Rumlaufen und das Fahrradfahren, mach ich alles alleine /l:ja/ muss ich auch haben /l:ja/ das brauch ich einfach, ich bin so (2) ich bin kein Gruppenmensch [...]" (Seite 11, Z. 29-31).

Diese Aussage steht jedoch im Widerspruch zu ihrer Teilnahme am Aquajogging, welches sie mit ihrem Mann und in einer Gruppe von anderen Seniorinnen und Senioren durchführt. Hier befürwortet sie den Gruppensport 
dank der guten Stimmung und der Geselligkeit in der Gruppe: „[...] da ist ja auch die Geselligkeit, ist eigentlich auch was Schönes beim Sport /l:ja/ also wenn man weiß, das ist ne geschlossene Gru-[...] und wir lachen viel, der [Übungsleiter] sagt immer, Lachen ist die beste Medizin //:lacht/ der will, bringt der uns immer zum Lachen, das ist auch gut /l:ja/ dass man es nicht so verkniffen sieht [...] äh (3) viele, viele äh, ältere Menschen sind ja ganz still /l:mhm/ und sie mal aus der Reserve zu locken, ist eigentlich ein gutes Zeichen beim Sport [...]" (Seite 13-14, Z. 47-49 \& 11-15).

Die zweite Ambivalenz in der Erzählung von Frau Schmal ist gekennzeichnet durch ihre psychische Gefühlssituation in puncto Sporttreiben. Einerseits weist sie ein stark depressives Verhalten auf, welches im deutlichen Kontrast zu aufkommenden Glücksgefühlen nach dem Sporttreiben steht. Sie beschreibt ihre allgemeine Gefühlslage seit ihrem körperlichen Rückschlag als gleichgültig und kaum veränderbar, behauptet jedoch, dass sie nicht unter Depressionen leidet: „[...] aber zum Glück habe ich keine Depressionen (1) [...] nicht, ich kann keine Depressionen mehr haben, ich, ich, weil ich immer ne gleichmäßige Stimmung habe /l:mhm/ ich kann nicht mehr wütend sein (1) ich kann mich zwar noch, noch freuen, aber ich merke nichts in meinem Körper so an, an echter Freude /l:mhm/ ich kann auch nicht mehr weinen, so richtig, so, so, ganz traurig sein (1) oder, so erhabene Augenblicke, das, das krieg ich alles nicht mehr mit (3) es ist alles so wie, also mein Vater hatte auf der Geige ein Dämpfer /I:mhm/ so'n, so'n, so'n kleinen Kamm, so'n kleiner Kamm war das /l:mhm/ und äh, so'n Dämpfer habe ich /l:mhm/ (1) äh, anders kann ich's Ihnen nicht beschreiben [...] und, und (1) ich versuche jeden Tag, äh, (1) über die Runden zu kommen [...]“ (Seite 9, Z. 24-35). Diese Erzählpassage wird unterstützt durch ihren leeren, ausdruckslosen Blick in die Ferne. Nur selten sucht sie den direkten Blickkontakt, macht viele Pausen und scheint lange nach den passenden Worten zu suchen.

Ihre einzige deutlich erkennbare Gefühlsveränderung, die sich auch in ihrem Lächeln während des Interviews widerspiegelt, erlebt sie allerdings nach dem Sporttreiben, wenn laut eigener Aussage Glückshormone ausgeschüttet werden, sie nass geschwitzt, kaputt und glücklich über die zurückgelegte Strecke (ob mit dem Fahrrad oder zu Fuß) ist, sodass hier die Vermutung nahe 
liegt, dass Sport für sie nicht nur ein gewisses Suchtpotenzial hat, sondern auch zum Zwang geworden ist: „[...] ja dieser innere Schweinehund, den habe ich noch gar nicht drüber gesprochen, den hat man ja sehr oft [...] wenn man denkt, o:h, es ist so schö:n, hast ne schöne $C D$ aufgelegt, und nachher trinkt man Tee, ich hab so schönen Kuchen gebacken, es ist eigentlich gemütlich hier /l:mhm/ und dann (1) nein, du musst noch raus /l:lacht/ aber, aber manchmal denke ich, oah nee (1) aber dann habe ich, ne Stunde später $n$ ganz blödes Gefühl /l:mhm/ äh, so'n, so'n (1) so'n duck-, Duckgefühl, so'n, so'n Aufgebegefühl hat man dann, das ist nie gut [...]" (Seite 13, Z. 5-12).

Am Ende des Interviews zeigt sich erneut, welchen Stellenwert das Sporttreiben in ihrer aktuellen Lebenssituation hat: „Ja halten Sie mir mal die Stange, dass ich, dass ich dabei blei:be jetzt bei dem, bei dem, bei diesem, bei dem, Tun /l:mhm/ dass ich nicht, nicht absacke und, und, gar nichts mehr mache, denn /l:ja/ das ist der Tod für mich [...]" (Seite 18, Z. 1-3). Hierbei ballt sie entschlossen die Fäuste und zeigt sich in ihrer Mimik fast verbissen. In ihrer Wortwahl lässt sich erkennen, dass sie das Ende ihrer sportlichen Aktivitäten mit ihrem Tod gleichsetzt. Ihre Abhängigkeit in Bezug auf sportliche Betätigung geht sogar so weit, dass sie ohne jene nicht mehr leben möchte und laut eigener Aussage auch nicht könnte.

Auch Frau Klum spricht sich in ihren Erzählungen gegen den Vereinssport bzw. gegen das Sporttreiben in der Gruppe aus, obwohl sie, wie Frau Schmal, ebenfalls im Verein in einer Gruppe Sport treibt. Ihr missfällt es, wenn sie bei der Gymnastik angesprochen wird, da sie sich auf die Bewegungen und ihren eigenen Körper konzentrieren möchte und während der Übungen nicht durch Gespräche, geschweige denn Tipps der anderen, gestört werden möchte: „[...] also, was ich hasse wie die Pest, wenn, äh, andere Teilnehmer ö von, von dieser Gruppen- äh, Gymnastik, mir dann irgendwie sagen, das musst du so und so machen /l:mhm/ das äh, von mir aus kann das ne Trainerin, machen, aber, nicht die Teilnehmerin, ich könnte, also verrückt werden, wenn die alten, Weiber, äh, /l:lacht/ mir sagen wollen, was ich als kleines Mädchen da, so ungefähr /l:ja/ äh, äh, zu tun habe und wo ich meine Matte hernehmen soll und, irgendwie so was, also irgendwie Vorschriften äh, lasse ich mir ungerne 
machen (1) [...]" (Seite 8, Z. 44-50). Beim Zitieren der anderen Teilnehmerinnen verstellt sie zudem ihre Stimme und stellt jene Tipps überspitzt dar.

Hinzu kommt, dass ein Vereinsbeitritt im fortgeschrittenen Alter die soziale Eingliederung zusätzlich erschwert und nur mit viel Eigeninitiative erreicht werden kann. „[...] also entweder, man gehört seit Jahren dazu [räuspert sich] und ähm (2) wenn man Kontakt zu den Leuten haben will, äh (1) muss man sich äh, sehr bemühen, aber das will ich überhaupt nicht, ich will da mein Sport machen und ich, will meinen Körper, meine Körper-Ertüchtigung (lacht) oder so was machen und äh, will da kein, äh, soziales Netzwerk haben (2) im Gegenteil [...] ich bin überhaupt kein Vereinsmensch und ich will meine Ruhe haben wenn ich, äh, zu Ende trainiert habe und fertig, ne /l:mhm/ und, entsprechend äh, reagieren natürlich die Leute auf mich [...]" (Seite 9, Z. 8-13 \& 29-31). Das häufige Räuspern Frau Klums ist laut eigener Aussage auf einen ihrer gesundheitlichen Rückschläge zurückzuführen, wodurch ihr das Sprechen und Formulieren jetzt schwerfällt.

Diese Einstellung erweckt auf den ersten Blick den Anschein, als läge Frau Klum generell nichts an sozialen Kontakten, doch ihre Freundschaften, wie sie selbst betont, hat sie außerhalb des Vereins im privaten Umfeld. Sportlich gesehen präsentiert sie sich als willensstark und stark gesundheitsmotiviert. Vor nicht allzu langer Zeit musste sie jedoch einen Rückschlag einstecken, welcher inrer Psyche immer noch negativ zusetzt: Auf einer, vom Verein organisierten Wanderung überschätzte sie ihre eigene Leistungsfähigkeit und muss seitdem ein traumatisches Erlebnis verkraften, weil sie aufgrund von körperlicher Erschöpfung die Wanderung abbrechen musste. Das Eingestehen dieser Niederlage fällt ihr sehr schwer, was sich auch an ihren Emotionen ablesen lässt, denn sie erkennt, dass sie gebrechlicher wird und nicht mehr die Strecken zurücklegen kann, die sie früher, ohne zu üben, zurücklegen konnte. „[...] und das [die Wanderung] hab ich einfach $\mathrm{n}$ bisschen unterschätzt, und äh, da hab ich nicht die zwei Tage durchgehalten, sondern bin äh, am ersten Tag, am Abend dann zurückgefahren (2) und es ging mir ga:nz schlecht /l:mhm/ ich hab geheult und ge- ((lacht)) es war furchtbar, es war frustrierend, weil ich gedacht habe, Gott Wandern, meine Güte, f- es gibt viele Leute, die 85 sind und, wandern und äh, schnurstracks /l:mhm/ und schneller als ich und so, und da 
hab ich gedacht, mein Gott, jetzt wirste wirklich alt, jetzt kannste das nicht mehr, aber (1) naja, muss man auch akzeptieren“ (Seite 6-7, Z. 45-2).

Hier wird deutlich, dass sie sich bei ihren Leistungen mit anderen Seniorinnen und Senioren vergleicht. Insbesondere die Tatsache, dass selbst ältere Teilnehmer/innen der Wanderung scheinbar mühelos die Strecke zurücklegten und sie hingegen aufgeben musste, erschweren ihr die Bewältigung dieser Situation. Am Ende des Zitates wechselt Frau Klum in die allgemeine ,man'Form und bezieht diese Situation eher auf die Allgemeinheit und nicht explizit auf sich selbst. Anhand dieses Beispiels lässt sich erahnen, dass sie das Negativerlebnis noch nicht komplett überwunden hat.

Grundsätzlich könnte diese hermeneutisch ausgewertete Thematik der Selbstpräsentation noch viel ausführlicher dargestellt werden, dann bestünde jedoch die Gefahr einer zu intensiven Fokussierung auf den Einzelfall bzw. eine biographische Detailliertheit, welche sich vom Schwerpunkt der Arbeit entfernen würde.

\section{Diskussion der Ergebnisse}

In diesem Abschnitt geht es nun darum, die gewonnenen Erkenntnisse aus den Ergebnissen erneut aufzugreifen und in Relation mit dem theoretischen Hintergrund zu bringen, um zu testen, inwieweit bereits bestehende Ergebnisse bestätigt oder neue bzw. weiterführende Aspekte erforscht wurden.

Mithilfe der qualitativen Interviews der Seniorinnen und Senioren sollte geprüft werden, wieso manche ältere Menschen trotz langer Sportabstinenz noch einmal beginnen, sich sportlich zu betätigen, während andere Seniorinnen und Senioren sportlich inaktiv bleiben.

Zunächst ist die Thematik des Sportbegriffes noch einmal zu beleuchten. Nachdem die vermeintliche Unachtsamkeit, nämlich die fehlende Vorabklärung des Sportbegriffes aufgetreten war, wurde bei der Analyse der Interviews 
schnell deutlich, dass das individuelle Verständnis der Definitionen von Sport als äußerst interessant zu klassifizieren ist. So lässt sich beobachten, dass einige der interviewten Seniorinnen und Senioren gar nicht genau wussten, was Sport eigentlich bedeutet bzw. eine falsche Vorstellung von diesem Begriff hatten. Daher kam es sowohl zu einer Unterschätzung des Sportbegriffes, indem die Interviewten zum Teil behaupteten, dass sie während der Gartenarbeit, dem Ausführen des Hundes oder beim Treppensteigen auf der Arbeit Sport trieben, als auch zu einer Überschätzung, indem sich eine falsche Vorstellung von Sport (z.B. Druck, Drill und Freudlosigkeit) etabliert hatte, sodass die Befragten von vornherein dem Sportbegriff sehr skeptisch gegenüberstanden. Darüber hinaus definierte eine weitere Gruppe von Befragten den Begriff gar nicht, sondern setzte ihr persönliches Sportverständnis als selbstverständlich voraus. Hier werden bereits individuelle Verhaltensmuster erkennbar, welche über die Persönlichkeiten und Hintergründe der Interviewten Auskunft geben.

Auch in der Literatur wird die Definition von Sport an sich kontrovers diskutiert. Aufgrund der Entwicklung von (Trend-)Sportarten in den letzten Jahrzehnten sowie einer immer größer werdenden Vereinsstruktur existiert nicht mehr der Sport, sondern eine Vielzahl von sportlichen Betätigungen, welche alle unter den Sportbegriff fallen. In dieser Arbeit handelt sportliche Betätigung, wie bereits im Kapitel 2.1 definiert, von "geplanten, bewusst aus dem Alltag herausgenommenen körperlichen Aktivitäten in der Freizeit, welche die Handlungsmuster des Sports übernehmen [...]" (Schlicht \& Schott 2013: 16). Die Betonung in diesem Zitat liegt auf ,bewusst aus dem Altag' und ,körperlichen Aktivitäten؛ Wenn alltäglich mit dem Fahrrad zum Einkaufen gefahren wird, ist dies nicht als sportliche Aktivität zu betiteln, sondern lediglich als Bewegung.

Ähnlich ist es auf der Homepage des Deutschen Olympischen Sportbundes (DOSB) vermerkt: „Die Ausübung der eigenmotorischen Aktivitäten muss Selbstzweck der Betätigung sein. Dieser Selbstzweck liegt insbesondere nicht vor bei Arbeits- und Alltagsverrichtungen und rein physiologischen Zustandsveränderungen des Menschen" 
Hier wird gleichermaßen betont, dass Tätigkeiten aus dem Altag nicht unter den Sportbegriff fallen. Dieser Teil der aufgeführten Definition von Sport wird für die Auswahl der Interviewteilnehmerinnen und -teilnehmer als entscheidend vorausgesetzt. Seniorinnen und Senioren, welche lediglich mit ihrem Hund spazieren gehen oder hin und wieder mit dem Fahrrad zu Freunden fahren, werden für die spätere Auswertung als ,Nicht-Sportler' charakterisiert, obwohl sie sich in ihrer Freizeit bewegen. Radfahren zählt lediglich in Fällen von bewusst geplanten Touren über einige Kilometer als sportliche Betätigung.

Dass die interviewten Seniorinnen und Senioren nicht in allen Fällen über ihre eigene Definition verfügen bzw. ihr eigenes Sportverständnis als selbstverständlich ansehen, ist für die Auswertung der Interviews nicht problematisch. Eine Definition ist nur notwendig, um für die wissenschaftliche Transparenz sorgen zu können und den Kontext oder theoretischen Rahmen des Sporttreibens festlegen zu können. Für individuelle sportliche Betätigungen ist Wissen über eine Definition des Sporttreibens nicht vonnöten.

Es ist offensichtlich, dass die Diskussion um den Sportbegriff an sich noch nicht abgeschlossen ist und stets Argumente für kleine Änderungen dieser Definition gefunden werden können. Vielleicht ist die exakte Festlegung auf den Sportbegriff und seine Bedeutung auch gar nicht der entscheidende Faktor: Wichtiger ist doch, dass Menschen sich bewegen und gerade in höherem Alter nicht in sitzende Verhaltensmuster verfallen. Solange die körperliche Aktivität regelmäßig und genügend oft pro Woche geschieht, ist nicht entscheidend, wie genau diese Aktivität aussieht, sondern eher dass sie durchgeführt wird.

Bei der Gruppe der Sporttreibenden ist diese Fragestellung noch auszuweiten auf den Punkt der Häufigkeit, hier macht nämlich die Anzahl der Betätigungen pro Woche den maßgebenden Unterscheid aus.

Wenn es in anderen empirischen Arbeiten um das Thema Sporttreiben im Kindes- und Jugendalter geht, wird hier häufig die Perspektive der Kinder und Jugendlichen eingenommen, das heißt, dass Studien mit Kindern und Jugendlichen selbst durchgeführt werden (vgl. u.a. Wagner 2011). Hier scheinen die Erfahrungen aus dieser Zeit aus der Sicht des späteren 
Erwachsenenalters nicht mehr als unbedingt relevant einzuordnen zu sein. Dass die Erlebnisse aus dem Kindes- und Jugendalter sehr prägend für den späteren Bezug zum Sporttreiben sein können, wurde in der Arbeit schon angeschnitten und wird nun bei der Diskussion zum Thema Schulsporterfahrungen erneut aufgegriffen.

In den Erzählungen der Interviewten von ihren Sporterfahrungen im Kindes-und Jugendalter teilen sich die Erlebnisse grob in zwei Lager: Zum einen gibt es die sportlich aktiven Kinder und Jugendlichen, welche sich schon während der Schulzeit neben dem Schulsport noch zusätzlich in Vereinen engagieren und hier auf Wettkampfebene agieren. Zum anderen existieren diejenigen, welche heutzutage mit Grauen auf die Schulsporterlebnisse in der Kindheit zurückblicken, da Schulsport stets negativ konnotiert war und sie daher so sehr abgeschreckt wurden und ein falsches Bild von sportlicher Betätigung bekamen, dass sie in ihrer Freizeit in keinem Fall noch zusätzlichen sportlichen ,Zwängen‘ ausgesetzt werden wollen und somit kein Bedürfnis nach Betätigung empfinden. Die Rolle des Schulsports wird im Folgenden noch konkreter thematisiert werden.

Zusätzlich zu den Sporterfahrungen im Kindes- und Jugendalter erzählen die Seniorinnen und Senioren über ihre aktuelle Sportpartizipation. Diese Erzählungen sind geprägt von Vorhaben und leeren Versprechungen einerseits sowie dem Vorschieben von Ersatzbetätigungen als eine Art Entschuldigung für mangelnde sportliche Betätigung andererseits. Dennoch ist sicherlich eine Ersatzbetätigung körperlicher Art einem völlig inaktiven Verhalten vorzuziehen, auch wenn es sich bei der Ersatzbetätigung nicht um die klassisch definierte sportliche Betätigung um der Bewegung selbst willen handelt. Ein körperlich aktiver Lebensstil, bestehend aus täglichem Zurücklegen gewisser Strecken mit dem Fahrrad oder zu Fuß sowie dem Arbeiten im Garten, ist zwar per se nicht als Sport zu bewerten, beugt jedoch trotzdem (insbesondere wenn die Seniorinnen und Senioren nicht mehr so körperlich fit sind) altersbedingten Abbauprozessen vor und trägt zum gesunden Altern bei. 
Als weiterer, dem Titel dieser Arbeit am nahesten erscheinender Punkt geht es um die Interpretation der Motive der Seniorinnen und Senioren zum Sporttreiben. Welches sind die eigentlichen Gründe, warum Menschen im hohen Alter noch einmal mit dem Sporttreiben beginnen? In der Literatur (vgl. Theorieteil Seite $7 \mathrm{ff}$.) steht der gesundheitliche Aspekt im Alterssport an erster Stelle. Danach trieben Seniorinnen und Senioren hauptsächlich Sport, um bereits bestehende Krankheitsbilder zu lindern oder potenziell entstehenden Krankheiten oder Abbauprozessen entgegenzuwirken. Diese Tendenz zeigt sich auch in dieser empirischen Untersuchung: Eine Gruppe von Seniorinnen und Senioren treibt lediglich Sport, weil ihr Arzt es als notwendig hervorgehoben hat, da sonst die körperlichen Einschränkungen eines Tages so groß würden, dass ein eigenständiges Leben im Alter nicht mehr möglich wäre. Inwiefern diese Art von Sporttreiben im Verlauf als angenehm oder zwanghaft empfunden wird, ist von Individuum zu Individuum unterschiedlich. Fest steht lediglich, dass alle Interviewten aus eigener Erfahrung berichten können, dass innen Bewegung gut tut - ob sie nun Spaß und Freude dabei empfinden oder nicht.

Ein zusätzlicher, häufig genannter Aspekt, welcher auch in der Theorie vorkommt, ist die Geselligkeit im Sporttreiben, also die Gemeinschaft in der Gruppe, die das Hauptmotiv der Seniorinnen und Senioren in manchen Fällen ausmacht. Insbesondere soziale Aktivitäten über das eigentliche Sporttreiben hinaus werden von den Interviewten als besonders schön hervorgehoben. Hierbei handelt es sich beispielsweise um Ausflüge am Wochenende, Weihnachts- und Geburtstagsfeiern.

Hier wird deutlich, dass die Gemeinschaft in der Gruppe auch über das Sporttreiben hinaus gepflegt werden kann und im Verlauf der gemeinsamen Aktivitäten flüchtige Bekannte zu guten Freunden werden können, welche immer vertrauter miteinander werden und sich auch außerhalb des Vereins oder der Sportgruppe privat verabreden.

Ein weiterer erfreulicher Nebeneffekt von gebildeten Freundschaften innerhalb der Sportgruppe ist der so genannte positive ,Gruppenzwang', also das Wissen, dass der Trainingspartner oder die Trainingspartnerin davon ausgeht, dass der Termin stets wahrgenommen wird und man inn nur in Notfällen ausfallen lassen 
würde. Hier steigert sich auch die Trainingsbeteiligung aufgrund der gegenseitigen Versprechungen.

Grundsätzlich muss jedoch hervorgehoben werden, dass es schwierig ist, bei Sporttreibenden ein einziges spezifisches Motiv auszumachen. Oftmals spielen mehrere Motive eine Rolle bzw. verändern sich auch manche Einstellungen dem Sport gegenüber im Verlauf der Durchführung. So kann es beispielsweise sein, dass der Wiedereinstieg auf Anraten eines Arztes initiiert wurde, die interviewte Person jedoch nach einer Weile sozial verankert ist und nun trotz verbessertem Gesundheitszustand nicht auf das wöchentliche Sporttreiben verzichten möchte.

Diese Tendenz der ineinander verschwimmenden Motive spiegelt sich auch in einigen anderen wissenschaftlichen Untersuchungen wider (vgl. hierfür z.B. die Cluster-Analyse von Sudeck, Lehnert \& Conzelmann (2011).). Nur selten herrscht lediglich ein einziges Motiv vor, welches zum Sporttreiben bewegt. Zwar gibt es sicherlich vorherrschende Motive, welche den Ursprungsanreiz zur sportlichen Betätigung geben, jedoch überlappen sich diese Anfangsmotive im Verlauf der Aktivitätsausübung zunehmend mit anderen Motiven. Umso schwieriger erscheint es daher, die Anfangsmotivation zu generieren, denn besteht bereits Motivation zum Sporttreiben, vermehren sich die Motive von Zeit zu Zeit leichter und andere Aspekte lassen das Sporttreiben als wichtigen Bestandteil des Alltags erscheinen. Existieren diese Motive jedoch nicht von Anfang an, muss sich die Grundeinstellung der Seniorinnen und Senioren der Thematik des Sporttreibens gegenüber verändern. Dies zu erreichen, stellt sich als keine leichte Aufgabe dar und so wird die Thematik der Grundeinstellung in Bezug zum Sporttreiben im Verlauf dieses Kapitels erneut aufgegriffen.

Darüber hinaus existieren in den Interviews auch Gründe seitens der Interviewten, die vom Sporttreiben abhalten. Zum einen herrschen selbst heutzutage noch falsche Vorstellungen vom Sporttreiben in Gruppen, das heißt, es gibt nicht genügend Aufklärung oder Transparenz über Inhalte in bestimmten Sportgruppen. So wird Sporttreiben beispielsweise von einer Interviewten ausschließlich mit Gewichtsreduktion assoziiert. Da sie keine Figur-Probleme hat, erscheint ihr Sport als überflüssig. 
Zum anderen sorgt eine lange Sportabstinenz in vielen Fällen für mangelndes Selbstbewusstsein, sodass Angst vor Blamage oder Scheu, den eigenen Körper in der Umkleidekabine zu zeigen, keine Seltenheit darstellen. Hinzu kommt die Tatsache, dass viele Sportgruppen in ihrer Konstellation schon seit Jahren bestehen und Neulinge zunächst Anschlussschwierigkeiten zu befürchten haben. Hier werden beispielsweise Erfahrungen geschildert, bei denen eine bestehende Seniorensportgruppe zusätzlich Ausflüge am Wochenende unternimmt und die neu beigetretenen Sportteilnehmerinnen und teilnehmer jedoch nicht dazu einlädt. Dass in einem solchen Fall das Gefühl von Akzeptanz und Eingliederung nicht vorhanden ist, erscheint nachvollziehbar. Nichtsdestotrotz hängt der Grad der Sozialisierung in der Gruppe auch von den Neueinsteigern ab: Wenn sich die Seniorinnen und Senioren ungern mit den „alten“ Teilnehmerinnen und Teilnehmern der Gruppe unterhalten, sich in anderen Umkleidekabinen umziehen oder zu Freizeitterminen bewusst nicht erscheinen, ist es nicht verwunderlich, dass die Integration nicht reibungslos verläuft.

Ein anderer Punkt sind die bereits erwähnten Ersatzbetätigungen oder schlichtweg andere Hobbys, zum Beispiel im musikalischen Bereich, welche die Freizeitgestaltung ausmachen. Hier geben die Interviewten oftmals an, für Sport nicht ausreichend Zeit zu haben. Inwieweit dies lediglich als Ausrede fungiert, ist schwer zu bestimmen. Zumindest im Rentenalter verfügen die Seniorinnen und Senioren über deutlich mehr Zeit als während ihrer aktiven Berufsphase.

Wie zuvor bereits angedeutet, wurde die Thematik um den Schulsport zusätzlich isoliert beleuchtet. Inwieweit spielen die Vorerfahrungen im Schulsport eine Rolle für das spätere Leben? Können Menschen mit negativen Schulsporterfahrungen im Seniorenalter dennoch leidenschaftlich Sport treiben oder ist dies eher unwahrscheinlich? Zur Beantwortung dieser Frage muss erneut ein Blick auf die Ergebnisse der Interviews geworfen werden. Zunächst ist als auffällig zu bewerten, dass nicht alle Interviewten in ihren Erzählungen einen Schwerpunkt auf den Schulsport legen. Manche der Interviewten erinnern sich kaum an ihren Schulsport oder halten eine ausführliche Erzählung für nicht notwendig. Hier lässt sich spekulieren, dass der Schulsport nicht in 
bedeutender Erinnerung geblieben ist, da er vermutlich keine hervorzuhebenden Erlebnisse enthielt und eher durchschnittlich war.

Doch auch positive Schulsporterfahrungen führen nicht automatisch zu einer lebenslangen Sportpartizipation. Wie in einem Interview deutlich wird, spielen auch die Eltern und ihre Einstellung dem Sport gegenüber eine wichtige Rolle. Fördern sie ihr Kind im Schulsportalter nicht bzw. verbieten sie einen starken Fokus auf eine Sportart, so kann es durchaus vorkommen, dass im höheren Alter die Leidenschaft für Sport deutlich reduziert ist (vgl. Interview mit Herrn Klaus).

Auch individuelle Abneigungen gegen bestimmte Sportarten ziehen sich oftmals durch den kompletten Lebenslauf. Wenn bereits im Schulsport Mannschaftssportarten abgelehnt wurden, so ist die Wahrscheinlichkeit, aus sozialen Motiven Sport zu treiben, auch im Seniorenalter als eher gering einzustufen (vgl. Interview mit Frau Klum).

Bei vielen interviewten Seniorinnen und Senioren war der Schulsport noch stark militärisch und von Körperertüchtigung geprägt. Diese Schulsporterfahrungen sind in allen Fällen negativ konnotiert und werden oftmals mit damaliger Wehrertüchtigung assoziiert. Dies geht einher mit einem eintönigen und einseitigen Schulsport: Ist der Schulsport nicht abwechslungsreich genug und bleiben keine positiven Erinnerungen, so lässt sich in den meisten Fällen aussagen, dass das spätere Sportinteresse stark gedämpft ist und oftmals andere Hobbys Vorrang dem Sport gegenüber finden.

Nichtsdestotrotz gibt es Ausnahmen von Interviewten, welche negative Schulsporterfahrungen vorzuweisen haben und dennoch heutzutage sportlich aktiv sind. Lediglich eine Seniorin im Felde der drei Ausnahmen betreibt ihre heutige sportliche Betätigung mit Leidenschaft und Spaß, während die anderen beiden Beispiele dieser Fälle eher zwangsweise aus gesundheitlichen Gründen Sport treiben und nicht notwendigerweise Freude daran empfinden. Hier zeigt sich, dass es in Ausnahmefällen doch vorkommen kann, dass negative Schulsporterfahrungen, vergessen“ werden können und durch neue sportliche Erlebnisse revidiert werden, sodass sich eine Leidenschaft für den Sport auch noch im hohen Alter entwickeln kann. Dennoch spielt der Schulsport eine der 
entscheidenden Rollen, was die spätere Sportpartizipation angeht. Diese Thematik ist in der Literatur noch nicht ausreichend untersucht. Hier ergeben sich für aufbauende Studien viele Möglichkeiten, die Korrelation von Schulsport und seine Auswirkungen auf die Sportpartizipation im Seniorenalter detailliert zu erforschen.

Die letzte große Kategorie aus dem Ergebnisteil ist die Selbstpräsentation der Interviewten. Diese lässt zwar auf den ersten Blick nicht auf die Motivation zum Sporttreiben schließen, ist jedoch enger durch persönliche Charakterzüge geprägt als zunächst angenommen. Die Selbstpräsentation der Interviewten ist von vielen Faktoren abhängig. Sie ist unter anderem gekennzeichnet durch Gestik und Mimik sowie durch sprachliche Besonderheiten.

Eine aufgeschlossene Person zeigt sich in ihrer Gestik und Mimik sehr lebendig. Sie hält beispielsweise ständig Blickkontakt, sucht Zuspruch und Reaktionen des Gegenübers und drückt viele Situationen mit Körpereinsatz aus, indem sie die Hände „sprechen“ lässt. Eher zurückhaltend erscheinende Personen richten ihren Blick in die Ferne, meiden Blickkontakt und lassen den Blick schweifen. Hinzu kommen häufig nervöse Handbewegungen wie etwa das Spielen mit den Fingernägeln oder Gegenständen auf dem Tisch.

Auch in der Sprache deutet sich diese Unsicherheit an, indem die Interviewten keinen natürlichen Redefluss generieren können, sondern eher stockend formulieren, viele kleine Pausen machen, sich oft versprechen und häufig ,äh“ oder ,öh' sagen. Insgesamt wirkt das Erzählte in diesen Fällen nicht flüssig und oftmals abgehackt.

Hier ist erneut auf die Wichtigkeit der exakten Transkription der Interviews hinzuweisen (vgl. z.B. Rosenthal 2005). Beim Zuhören und im Redefluss fallen Unsicherheiten und gelegentlich ein ,Äh' oder ,Öh' nicht deutlich auf. Steht schließlich jedoch das verschriftlichte Interview zur Verfügung, können diese Stellen beispielsweise farbig markiert werden und lassen sich so übersichtlich zählen oder einem Schema zuweisen. Auch die Pausenzeiten werden in der Transkription mit Hilfe von Zahlen für die Anzahl der Sekunden dargestellt (vgl. Transkriptionsregeln im Anhang). 
Zusätzlich lässt sich außerdem rein inhaltlich auf die Charaktere oder Intentionen der Interviewten schließen. Wird von den Interviewten beispielsweise offen geäußert, dass sie über gewisse Themen nicht sprechen möchten oder sie nichts mehr dazu sagen möchten, wird deutlich, dass sie sich auch hier der Interviewerin verschließen und nicht zu viel aus ihrem Privatleben preisgeben möchten. Hier ist es gerade für die Interviewerin wichtig, die Privatsphäre zu respektieren und nicht unnötig nachzufragen, wenn deutlich wird, dass die interviewte Person nicht über die Thematik sprechen möchte. Dies deckt sich mit den ,Regeln' des qualitativen Interviews (vgl. Rosenthal 2005).

Darüber hinaus lassen sich zwei Typen von interviewten Seniorinnen und Senioren ausmachen. Zum einen die glücklichen, lebensbejahenden und optimistischen Sporttreibenden, welche trotz zum Teil erheblicher gesundheitlicher Einschränkungen dankbar für ihre kleinen Fortschritte sind, welche sie körperlich erreichen. Das Sporttreiben sorgt jedoch nicht nur für ein gesteigertes körperliches Wohlbefinden, sondern gleichzeitig für ein verbessertes psychisches Wohlbefinden. Diese positive Stimmung wird erlangt dank spezieller Erlebnisse im Sportbereich. So kann bereits das Erreichen eines selbst gesteckten Zieles im Individualsportbereich für eine deutliche Stimmungshebung sorgen oder eine persönlich empfundene Steigerung der Beweglichkeit innerhalb der Gymnastikgruppe im Vergleich zur Vorwoche.

Hier bietet sich ein direkter Vergleich zur theoretischen Grundlage des Konzeptes der Salutogenese an (vgl. Antonovsky 1997). Die Seniorinnen und Senioren, welche trotz gesundheitlicher Rückschläge eine positive Lebenseinstellung vorweisen, befinden sich zwar aufgrund ihrer Einschränkungen auf den ersten Blick eher auf der negativen Seite des Gesundheits-Krankheits-Kontinuums, kompensieren aber diese negative Voraussetzung durch ihr positives Kohärenzgefühl. Deshalb rücken sie auch auf dem Kontinuum mehr zum positiven Pol, da sie die sportliche Betätigung als sinnvoll erachten, weil diese innen hilft, ihre körperlichen Probleme zu reduzieren.

Die Analyse der Interviews ergibt, dass die Grundeinstellung zum Leben bei den sporttreibenden Seniorinnen und Senioren deutlich positiver zu bewerten 
ist als bei den nicht sporttreibenden. Diejenigen, welche dem Sport gegenüber eher abgeneigt sind und sich nicht körperlich betätigen, präsentieren ihre Rolle und ihren gesundheitlichen Status in einigen Fällen als die eines Opfers. So wirken private Schicksalsschläge stets negativ auf die Psyche der Seniorinnen und Senioren. Nach einer gewissen Verarbeitungsphase schaffen es nur manche, diese schwierige Situation zu überwinden. Dies gelingt in den meisten Fällen dank einer positiven Lebenseinstellung und der Hilfe der sportlichen Betätigung, die sowohl körperliche Einbußen reduziert als auch psychische Besserung evozieren kann. Andere bleiben jedoch in ihrer Opferrolle und zeigen in einigen Fällen sogar Verhaltensmuster, die einer Depression ähneln. Hier wäre sportliche Betätigung ein ganz wichtiger Baustein gegen die negativen Assoziationen mit und Auswirkungen von Schicksalsschlägen. Dennoch ist die Vermittlung dieser Zusammenhänge selbst für einen Arzt keine leichte Aufgabe.

Doch auch Ambivalenzen von leeren Versprechungen sich selbst gegenüber oder Vorhaben, die nicht in die Tat umgesetzt wurden, werden innerhalb der Inhaltsanalyse der Interviewten deutlich. Sind sie in der Theorie noch euphorisch und schwärmen von bestimmten sportlichen Tätigkeiten, können sie diese schließlich in der Praxis aus einer Vielzahl von selbst ausgedachten Gründen nicht ausführen. Hier mangelt es daher an der Umsetzung in die Praxis, meist aus Gründen der Willensschwäche.

Überdies ist die Überwindung, nach einer langen Sportabstinenz einem Verein beizutreten, häufig sehr hoch. Hier ist der Charakter der Seniorinnen und Senioren der ausschlaggebende Punkt: Ist eine Person eher aufgeschlossen und kontaktfreudig, hat diese einen leichteren Zugang zu fremden Gruppen. Die Integration in die Gruppe vollzieht sich schneller und Kontakte werden effektiver geknüpft. Die Person muss jedoch selbst aktiv sein, um diese Ziele zu erreichen. Nur wenn aktiv versucht wird, sich in die Gruppe einzugliedern, ist das Vorhaben erfolgversprechend.

Diejenigen Seniorinnen und Senioren, welche Bedenken haben, einer neuen Gruppe beizutreten, stoßen oftmals schon aufgrund ihrer Grundeinstellung auf Probleme. Sie nehmen an, dass die Gruppe schon seit Jahren in dieser Konstellation zusammen ist und daher Fremde ungern gesehen sind. Anschluss 
zu finden gestaltet sich schwieriger, wenn eine Skepsis von vornherein vorhanden ist. Damit einher geht ein passives Auftreten seitens der Neulinge. Präsentieren sich die Seniorinnen und Senioren, welche neu in eine Gruppe kommen, eher schüchtern und zurückhaltend, ist die Integration in die neue Gruppe schwieriger. Dies hält viele Seniorinnen und Senioren davon ab, den Schritt zu wagen und neu in eine bereits langjährig bestehende Gruppe einzutreten. Hinzu kommen Zweifel wegen der eigenen körperlichen Leistungsfähigkeit, Ängste vor dem Präsentieren des eigenen, womöglich adipösen Körpers und Scheu, Übungen falsch oder gar nicht ausführen zu können.

Hier muss in Zukunft auch seitens der Vereine umgedacht werden. Als eine Möglichkeit bietet sich ein breiteres Angebot für Neueinsteiger an, welches explizit darauf hinweist, dass Gruppen neu geformt werden und damit sowohl das Problem der sozialen Isolation als auch das Problem der Scheu vor eigenem körperlichem Misserfolg gedämpft werden. Die Hürde der Mitgliedschaft in Vereinen kann beispielsweise durch den Verkauf von Zehnerkarten oder dem Anbieten von kostenlosen Schnuppertrainings reduziert werden. So wären die Teilnehmenden nicht zu sehr dem Zwang der regelmäßigen Teilnahme ausgesetzt, sondern könnten zunächst ausprobieren, ob sie sich in der Sportart und Gruppe wohlfühlen. In Teilen wird dieser Ansatz bereits von einigen Vereinen und dem DOSB als Vorreiter umgesetzt (vgl. www.dosb.de).

Außerdem besteht eindeutig Bedarf nach mehr Transparenz bezüglich der Inhalte der einzelnen Kurse, welche von Vereinen insbesondere für Seniorinnen und Senioren angeboten werden. Nach Auswertung der Interviews reicht es für viele der Interviewten nicht, wenn ein Verein einen Kurs namens ,Pilates“ anbietet, wenn Inhalte dieses Kurses dadurch nicht transparent werden. Die zum Teil unbekannten Namen der Kurse wirken in diesen Fällen eher abschreckend und die Hürde der Teilnahme ist umso größer.

Auch auf Seiten der Fitnessstudios ist eine breitere Werbekampagne vonnöten, um insbesondere Seniorinnen und Senioren für eine Mitgliedschaft zu werben, welche sportlich inaktiv sind und es auch lange waren. Hier bietet es sich ebenfalls an, sich noch mehr und konkreter auf die Zielgruppe der älteren 
Menschen zu konzentrieren. Zwar gibt es schon einige Fitnessstudios, welche ausschließlich Seniorinnen und Senioren aufnehmen ${ }^{4}$, jedoch wird das Sporttreiben im Fitnessstudio vornehmlich immer noch mit dem Muskelaufbau jüngerer Leute assoziiert. Nur durch direktes Ansprechen der möglicherweise interessierten Seniorinnen und Senioren über Flyer oder auf Anraten eines Arztes rückt das Training in Fitnessstudios in den Fokus der Aufmerksamkeit. Sonst haben die älteren Menschen heutzutage eher noch keinen Zugang zum Fitnessstudio, es sei denn, in ihrem Bekanntenkreis oder in der eigenen Familie wird dafür geworben und Vorbilder existieren. Vielleicht wird sich der Trend bezüglich der Trainingsbeteiligung in Fitnessstudios in den kommenden Jahren auch für ältere Menschen deutlich weiter fortsetzen, da dann zum einen ein anderes Bild vorherrschen wird, wenn automatisch mehr ältere Menschen Gäste in Fitnessstudios sind, und zum anderen die Generation, welche heutzutage im mittleren Erwachsenenalter ist und bereits Vorerfahrungen aus dem Fitnessstudio hat, ihre Gewohnheiten vermutlich nicht ändern wird.

Alle weiteren möglichen Barrieren, welche in Kapitel 3.7 bereits angesprochen wurden, wie z.B. schlechtes Wetter oder mangelnde Ausstattung der Sportstätte sowie das Vorhandensein anderer Hobbys, können nur dann überwunden werden, wenn bereits ein gewisses Grundinteresse der sportlichen Betätigung gegenüber besteht. Trotz aller Bemühungen und Ideen ist es jedoch illusorisch, jeden einzelnen Menschen zum Sporttreiben bis ins hohe Alter zu motivieren. Die steigenden Mitgliederzahlen der Seniorinnen und Senioren in Vereinen oder Fitnessstudios zeigen aber, dass das Konzept des Seniorensports fruchtet und mit den oben genannten Ideen möglicherweise in Zukunft einen noch höheren Anteil an älteren Menschen dazu bewegen kann, sich sportlich zu betätigen.

\footnotetext{
${ }^{4}$ Vgl. z.B. http://www.vitalis-muenchen.de/fitness/fit-fuer-die-enkel.html
} 


\section{$14 \quad$ Fazit und Ausblick}

Zum Abschluss soll nun eine möglichst prägnante Antwort auf die in der Einleitung formulierte Hauptfragestellung gegeben werden. ,Wie kommt es, dass manche Menschen im hohen Alter mit dem Sporttreiben beginnen, während andere Menschen inaktiv bleiben?' Zur Beantwortung dieser Frage können vier Aspekte hervorgehoben werden. Zunächst ist die Rolle der Gesundheit von hoher Bedeutung: Wenn ein Arzt einer Patientin oder einem Patienten dringend empfiehlt, mit dem Sporttreiben zu beginnen, weil sonst gravierende körperliche Folgen entstehen könnten, befolgt die Mehrheit der Patientinnen und Patienten diesen Rat. In diesem Fall beginnen also tendenziell eher Menschen mit körperlichen Einschränkungen mit dem Sporttreiben als jene, welche keine Probleme haben.

Des Weiteren spielen soziale Aspekte eine wichtige Rolle in Bezug auf die Sportpartizipation. Insbesondere in Vereinen oder Gruppenkursen im Fitnessstudio ist die soziale Komponente ein ebenfalls wichtiger Faktor, welcher zum Sporttreiben anregt. Die Gemeinschaft in der Gruppe verbindet die Teilnehmenden, sodass Freundschaften entstehen und Aktivitäten über das Sporttreiben hinaus geplant und durchgeführt werden. Hier beginnen also tendenziell eher kontaktfreudige Menschen mit dem Sporttreiben, welche auf der Suche nach einem neuen sozialen Umfeld sind als diejenigen, die bereits aufgrund von anderen Hobbys oder anderen Umständen in schon bestehenden Freundeskreisen verankert sind.

Überdies, und hier entsteht ein neuer Aspekt im wissenschaftlichen Diskurs, sind sportliche Vorerfahrungen essentiell. Positive Schulsport-erfahrungen sorgen in der Regel auch im hohen Erwachsenenalter eher für eine freiwillige Sportpartizipation, während negative Erfahrungen im Schulsport in den meisten Fällen für eine Abneigung sportlicher Betätigung im Allgemeinen sorgen. Zudem fungieren die Eltern bzw. die engen Bekannten und Freunde als Vorbilder in vielen Bereichen. Somit ist auch hier entscheidend, ob Eltern ihren Kindern ein sportliches Leben vormachen oder selbst passiv sind und dem Sport keine hohe Wichtigkeit zuweisen. In vielen Fällen überträgt sich eine 
gewisse Grundhaltung dem Sport gegenüber von der Elterngeneration auf die Kindergeneration.

Als letzter Aspekt wird die generelle Veränderung, welche mit Eintritt in das Seniorenalter beginnt, beleuchtet. Oftmals ist der Eintritt in das Seniorenalter zeitlich eng verknüpft mit dem Beginn des Ruhestandes. Der Beginn des Ruhestandes sorgt sowohl im sozialen Bereich als auch in der täglichen Lebensplanung für extreme Veränderungen. Zum einen besteht das soziale Umfeld nicht mehr aus Arbeitskolleginnen und -kollegen, sodass ein großer Anteil des Umfeldes plötzlich stark in den Hintergrund rückt. Zum anderen entsteht durch den Ruhestand auf einmal die Möglichkeit einer komplett anderen Tagesplanung, da deutlich mehr Zeit für die Freizeitgestaltung bleibt. Hier beschließen einige Seniorinnen und Senioren erneut mit dem Sporttreiben zu beginnen, da sie nun zeitlich viel freier wählen können, wann sie an Aktivitäten teilnehmen. Andere Rentnerinnen und Rentner hingegen nehmen den Begriff des Ruhestandes eher wörtlich und beschränken sich auf ruhigere Hobbys oder andere inaktive Tätigkeiten.

Bei der genaueren Betrachtung der Analyse der dem Sport abgeneigten Gruppe von Seniorinnen und Senioren treten ebenfalls neue Aspekte hervor, die in diesem Rahmen in der bestehenden Literatur zu ähnlicher Thematik nicht vorzufinden sind.

Die Gründe von Nicht-Sportlerinnen und -Sportlern, welche genannt werden, um das Nichtsportlerleben zu untermauern, ähneln interessanterweise sehr stark den Gründen für das Sporttreiben - lediglich in umgekehrter Bedeutung: Ein oftmals genannter Aspekt, welcher gegen das Sporttreiben spricht, ist die Gesundheit. Einige Interviewte fühlen sich körperlich ausreichend gesund und sind somit der Meinung, dass sie sportliche Betätigung nicht notwendigerweise benötigen. Dass Sport jedoch insbesondere als präventives Mittel von unbezahlbarem Wert ist, leuchtet nicht allen von innen ein. Oft wird die Denkweise in vielen Fällen erst umgestellt, wenn persönliche gesundheitliche Einschränkungen bereits vorliegen und nicht schon im Voraus, um mögliche pathogenetische Veränderungen zu verhindern. 
Auch im Hinblick auf die soziale Komponente ergeben sich in der Argumentation Parallelen. Die Nicht-Sportlerinnen und -Sportler sehnen sich oftmals nicht nach sozialer Anbindung in Vereinen oder anderen Sportgruppen, da sie beispielsweise in musikalischen Einrichtungen soziale Kontakte haben oder grundsätzlich über einen großen Freundeskreis verfügen. Anderen Seniorinnen und Senioren erscheint der Aspekt der sozialen Interaktion grundsätzlich nicht wichtig zu sein. So kann es auch vorkommen, dass sich Alleinstehende oder auch vereinzelt Ehepaare abgrenzen und eher zurückgezogen leben.

Um in Zukunft eine höhere Anzahl von Seniorinnen und Senioren zur sportlichen Betätigung motivieren zu können, bedarf es einer noch intensiveren Aufklärung über präventive Maßnahmen zur Gesunderhaltung, z.B. durch Gesundheitsprogramme. Nur wenn ältere Menschen den Sinn und das Ziel der sportlichen Betätigung verstehen und evtl. auch durch abschreckende Beispiele darauf aufmerksam gemacht werden, wie immens wichtig Bewegung im Alter ist, können sich weitere Erfolge abzeichnen. Darüber hinaus ist eine erhöhte Transparenz bezüglich der genauen Inhalte von Sportkursen vonnöten. Insbesondere Wiederanfängerinnen und -anfänger, welche jahrelang keinen Zugang zur sportlichen Betätigung hatten, sind oftmals nicht über moderne Kursbezeichnungen oder Sportarten informiert und werden somit durch unbekannte Betitelungen eher abgeschreckt.

Je mehr Menschen im hohen Alter Sport treiben, desto mehr werden sich mit erhöhter Wahrscheinlichkeit von der Mehrheit beeinflussen lassen und mit Bekannten oder Verwandten mitgehen, um gemeinsam Sport zu treiben. Das langfristige Ziel sollte sein, dass eines Tages Sport idealerweise als Selbstverständlichkeit in den Alltag integriert ist, sodass sportliche Betätigung als tägliche Routine durchgeführt wird.

Dass diese Wunschvorstellung nicht für jeden Menschen zutreffen wird, ist wahrscheinlich, jedoch ist es bereits ein großer Erfolg, wenn in Zukunft der Anteil der sporttreibenden Seniorinnen und Senioren kontinuierlich ansteigt. 
Hier ist abschließend erneut die in dieser Arbeit beleuchtete Wichtigkeit des Schulsports im Hinblick auf die weitere sportliche Zukunft hervorzuheben. Die meisten Menschen (insbesondere im unsportlichen Umfeld) kommen erst im Schulalltag mit dem Sporttreiben in Kontakt. Der Sportunterricht wurde von einer Vielzahl der Interviewten als prägend und richtungsweisend empfunden. Wenn im Schulsport bereits Negativerfahrungen gemacht werden, stehen die Vorzeichen für eine sportliche Lebensweise tendenziell schlecht.

Daher sollte in weiteren möglichen Studien ein starker Fokus auf den als so entscheidend und prägend einzustufenden Schulsport in Kombination mit der weiteren Biographie und dem Sporttreiben im höheren Alter gelegt werden. Nur so lassen sich fundierte empirische Daten erheben, welche generalisierte Aussagen und nicht nur Hypothesen oder Tendenzen ermöglichen. Mithilfe dieser Arbeit wird ein Schritt in die richtige Richtung getan, doch das wissenschaftliche Forschungsfeld ist längst nicht erschöpft.

Des Weiteren könnte in Folgestudien der Zusammenhang von Bildung und aktiver Sportpartizipation näher beleuchtet werden - ein Aspekt, auf den in dieser Arbeit nicht näher eingegangen wurde.

Darüber hinaus bietet es sich an, in nachfolgenden Forschungsarbeiten gezielte Maßnahmen zu untersuchen, um inaktive Seniorinnen und Senioren zum Sporttreiben zu bewegen. Hier wäre eine noch engere Kooperation zwischen Vereinen, Fitnessstudios und Krankenkassen wünschenswert, welche gemeinsam über Broschüren, Informationsveranstaltungen, Bonuskurse oder Schnupperkurse auf abwechslungsreiche sportliche Aktivitäten aufmerksam machen könnten. Trotz aller noch bestehenden Defizite ist die immer stärker werdende Förderung des Seniorensports auf einem guten Weg. Auch dies ist eine notwendige Maßnahme, um den Folgen des demographischen Wandels zu begegnen. 


\section{Literaturverzeichnis}

Abuhamdeh, S. \& Csikszentmihalyi, M. (2012). Attentional involvement and intrinsic motivation. In: Motivation and Emotion, Vol. 36: 257-267.

Achtziger, A. \& Gollwitzer, P.M. (2006). Motivation und Volition im Handlungsverlauf. In: Heckhausen, H. \& Heckhausen. J. (Hrsg.) (2006³). Motivation und Handeln. Heidelberg: 277-301.

Antonovsky, A. (1997). Salutogenese: Zur Entmystifizierung der Gesundheit. Tübingen.

Backes, G. (2001). Aktivität vs. Rückzug im Alter - gesellschaftliche Möglichkeiten und Grenzen. In: Daugs, R. et al. (Hrsg.) (2001). Aktivität und Altern. Schorndorf: 289-304.

Baur, J., Bös, K., Conzelmann, A. \& Singer, R. (Hrsg.) (2009²). Handbuch motorische Entwicklung. Schorndorf.

Becker, S. \& Schneider, S. (1995). Analysen zur Sportbeteiligung auf der Basis des repräsentativen Bundes-Gesundheitssurveys 1998: Ausmaß und Korrelate sportlicher Betätigung bei bundesdeutschen Erwerbstätigen. In: Sport und Gesellschaft, 2 (2): 173-204.

Beckmann, J. Fröhlich, S. M. \& Elbe, A.-M. (2009). Motivation und Volition. In: Schlicht, W. \& Strauß, B. (Hrsg.) Grundlagen der Sportpsychologie. Göttingen.

Brand, R. (2006). Die affektive Einstellungskomponente und ihr Beitrag zur Erklärung von Sportpartizipation. In: Zeitschrift für Sportpsychologie, 13 (4): 147-155.

Brière, N.M. et al. (1995). Développement et validation d'une mesure de motivation intrinsèque, extrinsèque et d'amotivation en contexte sportif : L'Echelle de Motivation dans les Sports (EMS). In: International Journal of Sport Psychology, Vol. 26: 465489.

Bundesministerium des Innern (2011). Demografiebericht. Bericht der Bundesregierung zur demografischen Lage und künttigen Entwicklung des Landes. Berlin.

Bundesministerium des Innern (2012). Jedes Alter zählt. Demografiestrategie der Bundesregierung. Berlin.

Bundesministerium des Innern (2013). Jedes Alter zählt. Zweiter Demografiegipfel der Bundesregierung am 14. Mai 2013. Berlin.

Carius, D. (2012). Motorische Präzisionsleistungen in der zweiten Lebenshälfte Altersbezogene Einflussfaktoren der Fertigkeitsaneignung. Dissertation. Universität Halle-Wittenberg. 
Cohen-Mansfield, J., Marx, M.S., Guralnik, J.M. (2003). Motivators and barriers to exercise in an older community-dwelling population. In: Journal of Aging and Physical Activity: 11: 242-253.

Conzelmann, A. (1997). Entwicklung konditioneller Fähigkeiten im Erwachsenenalter. Wissenschaftliche Schriftenreihe des Deutschen Sportbundes, Band 29. Schorndorf.

Conzelmann, A. \& Blank, M. (2009). Entwicklung der Ausdauer. In: Baur, J., Bös, K., Conzelmann, A. \& Singer, R. (Hrsg.) (2009²). Handbuch motorische Entwicklung. Schorndorf: 167-186.

Dahlhaus, J. (2007). Motivierung zum Alterssport. Von Metatheorien zum fundierten Praxiskonzept. Saarbrücken.

Daugs, R. et al. (Hrsg.) (2001). Aktivität und Altern. Schorndorf.

Dean, R.N. et al. (2007). Testing the efficacy of the theory of planned behaviour to explain strength training in older adults. In: Journal of Aging and Physical Activity, 15 (1): $1-12$.

DeFina, L.F. et al. (2013). The association between midlife cardio-respiratory fitness levels and later-life dementia: a cohort study. In: Annals of Internal Medicine. Vol. 158 (3): 162-68.

Delshad, M. et al. (2013). Effect of Strength Training and Short-Term Detraining on Muscle Mass in Women Aged Over 50 Years Old. In: International Journal of Preventive Medicine, Vol. 4(12): 1386-94.

Denk, H., Pache, D. \& Schaller, H.J. (Hrsg.) (2003). Handbuch Alterssport. Schorndorf.

Denk, H. \& Pache, D. (2003). Gesellschaftliche und inhaltliche Rahmenbedingungen von Alterssport. In: Denk, H., Pache, D. \& Schaller, H.J. (Hrsg.) (2003). Handbuch Alterssport. Schorndorf: 23-96.

De Pero, R. et al. (2009). Motivation for sport participation in older Italian athletes: the role of age, gender and competition level. In: Sports Science Health, Vol. 5: 61-69.

Diekmann, A. (2010, 4. Auflage). Empirische Sozialforschung. Grundlagen, Methoden, Anwendungen. Hamburg.

Eichberg, S. \& Mechling, H. (2009). Motorische Entwicklung im höheren Erwachsenenalter. In: Schlicht, W. \& Strauß, B. (Hrsg.) (2009). Grundlagen der Sportpsychologie. Göttingen: 333-348.

Engels, U. (2004). Studienabbruch als biographische Arbeit? Eine biographietheoretische Untersuchung im Fach Sport / Sportwissenschaften. Dissertation Universität Göttingen. 
Eriksson, M. \& Lindström, B. (2007). Antonovsky's sense of coherence scale and its relation with quality of life: A systematic review. In: Journal of Epidemiology and Community Health, Vol. 61 (11): 938-944.

Feland, J., Hager, R. \& Merrill, R. (2005). Sit to stand transfer: Performance in rising power, transfer time and sway by age and sex in senior athletes. In: British Journal of Sports Medicine, 39 (11): e39.

Flensborg-Madsen, T., Ventegodt, S., \& Merrick, J. (2006). Sense of coherence and physical health. Testing Antonovsky's theory. In: The Scientific World Journal, Vol. 6: 2212-2219.

Flick, U. (1996). Qualitative Forschung. Ein Handbuch. Reinbek bei Hamburg.

Fröhlich, S.M. \& Kuhl, J. (2003). Das Selbststeuerungsinventar: Dekomponierung volitionaler Funktionen. In: Stensmeier-Pelster, J. \& Rheinberg, F. (Hrsg.) (2003). Diagnostik von Motivation und Selbstkonzept. Göttingen: 221-257.

Froschauer, U. \& Lueger, M. (2003). Das qualitative Interview. Wien.

Fuchs, R. (1997). Psychologie und körperliche Bewegung. Göttingen.

Fuchs, R. (2003). Sport, Gesundheit und Public Health. Göttingen.

Gabler, H. (2000). Kognitive Aspekte sportlicher Handlungen. In: Gabler, H., Nitsch, J.R. \& Singer, R. (2000). Einführung in die Sportpsychologie. Teil 1: Grundthemen. Schorndorf.

Gabler, H. (2000). Motivationale Aspekte sportlicher Handlungen. In: Gabler, H., Nitsch, J.R. \& Singer, R. (2000). Einführung in die Sportpsychologie. Teil 1: Grundthemen. Schorndorf.

Gabler, H. (2002). Motive im Sport. Motivationspsychologische Analysen und empirische Studien. Schorndorf.

Geißler, R. (2004). Facetten der modernen Sozialstruktur. In: Informationen zur politischen Bildung 269, überarbeitete Neuauflage 2004: 75-76.

Gillet, N., Vallerand, R.J. \& Rosnet, E. (2009). Motivational clusters and performance in a real-life setting. In: Motivation and Emotion, Vol. 33: 49-62.

Gillet, N. et. al (2013). The mediating role of positive and negative affect in the situational motivation-performance relationship. In: Motivation and Emotion, Vol. 37: 465-479.

Gläser, J. \& Laudel, G. (2006²). Experteninterviews und qualitative Inhaltsanalyse. Wiesbaden.

Gordon, C.D., Wilks, R. \& Mc Caw-Binns, A. (2013). Effect of aerobic exercise (walking) training on functional status and health-related quality of life in chronic 
stroke survivors: A randomized controlled trial. In: Stroke; A Journal of Cerebral Circulation. Vol. 44 (4): 1179-81.

Guay, F., Vallerand, R.J. \& Blanchard, C. (2000). On the Assessment of Situational Intrinsic and Extrinsic Motivation: The Situational Motivation Scale (SIMS). In: Motivation and Emotion, Vol. 24/3: 175-213.

Hagger, M. \& Chatzisarantis, N. (2008). Self-Determination Theory and the psychology of exercise. In: International Review of Sport and Exercise Psychology, Vol 1/1: 79-103.

Heckhausen, H. (1989). Motivation und Handeln. Berlin.

Heckhausen, J. \& Heckhausen, H. (Hrsg.) (2006). Motivation und Handeln. Heidelberg.

Helfferich, C. (2005²). Die Qualität qualitativer Daten. Manual für die Durchführung qualitativer Interviews. Wiesbaden.

Hepler, J., Wang, W. \& Albarracin, D. (2012). Motivating exercise: The interactive effect of general action goals and past behavior on physical activity. In: Motivation and Emotion, Vol. 36: 365-370.

Hepler, J. et. al (2012). Being active and impulsive: The role of goals for action and inaction in self-control. In: Motivation and Emotion, Vol. 36: 416-424.

Hirtz, P. (1994). Koordinative Fähigkeiten. In: Schnabel, G., Harre, D. \& Borde, A. (Hrsg) (1994). Trainingswissenschaft. Berlin: 137-146.

Huber, G. (2011). Fehlende Motivation - eine Folge der Evolution? Bewegung als möglicher evolutionärer Schlüsselfaktor zur Entstehung des Übergewichts. In: Schüle, K. \& Huber, G. (Hrsg.) Bewegungstherapie und Gesundheitssport, 27 (3), 107-109.

Joshua, A.M. et al. (2014). Effectiveness of Progressive Resistance Strength Training Versus Traditional Balance Exercise in Improving Balance Among the Elderly - A Randomised Controlled Trial. In: Journal of Clinical and Diagnostic Research, Vol. 8(3): 98-102.

Keller, S. (2015). Seniorenleistungssport. Motive und Einstellungen. Hildesheim.

Kolb, M. (1999). Bewegtes Altern. Schorndorf.

Kolt, G.S., Driver, R.P. \& Giles, L.C. (2004). Why older Australians participate in exercise and sport. In: Journal of Aging and Physical Activity, Vol. 12(2): 185-98.

Kruse, A. (2001). Der Beitrag der Erwachsenenbildung zur Kompetenzentwicklung im Alter. In: Zeitschrift für Erziehungswissenschaft, Vol. 4: 555-575.

Küsters, I. (2006). Narrative Interviews. Grundlagen und Anwendungen. Wiesbaden. 
Kuckartz, U. (2012). Qualitative Inhaltsanalyse. Methoden, Praxis, Computerunterstützung. Weinheim und Basel.

Lapointe, M.-C. \& Perreault, S. (2013). Motivation: Understanding leisure engagement and disengagement. In: Society and Leisure, Vol. 36/2: 136-144.

Lee, I.H. \& Park, S.Y. (2013). Balance Improvement by Strength Training for the Elderly. In: Journal of Physical Therapy Science, Vol. 25(12): 1591-93.

Leigey, D. et al. (2009). Participation in High-Impact Sports Predicts Bone Mineral Density in Senior Olympic Athletes. In: Sports Health. Vol. 1(6): 508-513.

Lindström, B. \& Eriksson, M. (2005). Salutogenesis. In: Journal of Epidemiology and Community Health, Vol. 59 (6): 440-442.

Lucius-Hoene, G. \& Deppermann, A. (2002). Rekonstruktion narrativer Identität. Ein Arbeitsbuch zur Analyse narrativer Interviews. Opladen.

Mallett et al. (2007). Sport motivation scale-6 (SMS-6): A revised six-factor sport motivation scale. In: Psychology of Sport and Exercise, Vol. 8: 600-614.

Mayring, P. (2010). Qualtitative Inhaltsanalyse. Grundlagen und Techniken. 11., aktualisierte und überarbeitete Auflage. Weinheim und Basel.

Meusel, H. et al. (Hrsg.) (1980). Dokumentationsstudie: Sport und Alter. Schorndorf.

Müller, N., Rösch, H.-E. \& Wischmann, B. (Hrsg.) (1979). Alter und Leistung. Hochheim.

Nitsch, J.R. (2000). Handlungstheoretische Grundlagen der Sportpsychologie. In: Gabler, H., Nitsch, J.R. \& Singer, R. (2000). Einführung in die Sportpsychologie. Teil 1: Grundthemen. Schorndorf.

Oschütz, H. \& Belinová, K. (2003). Training im Alter. In: Denk, H., Pache, D. \& Schaller, H.J. (Hrsg.) (2003). Handbuch Alterssport. Schorndorf: 147-198.

Pelletier, L. G et al. (1995). Toward a new measure of intrinsic motivation, extrinsic motivation, and amotivation in sports: The Sport Motivation Scale (SMS). Journal of Sport \& Exercise Psychology, 17: 35-53.

Perrig-Chiello, P. (2008). Wohlbefinden und Gesundheit. In: Mess, F., Dugandzic, D. \& Woll, A. (Hrsg.) (2008). Erfolgreiches Altern durch Sport. Konstanz: 35-59.

Pfeffer, I. (2010). Motivation zur Verhaltensänderung im gesundheitsorientierten Sport. Effekte einer psychologischen Intervention in 12-wöchigen Ausdauersportkursen. Berlin.

Przyborski, A. \& Wohlrab-Sahr, M. (2010³). Qualitative Sozialforschung. München.

Radzik-Bolt, D. (2006). Gesichter des Glücks. Glück und Unglück im Lebensrückblick alter Menschen. Dissertation Universität Zürich. 
Rao, S. S. (2005). Prevention of Falls in Older Patients. In: American Family Physician Vol. 72 (1): 81-88.

Reed, C.E. \& Cox, R.H. (2007). Motives and regulatory style underlying senior athletes' participation in sport. In: Journal of Sport Behavior, Vol. 30(3): 307ff.

Reed-Jones et al. (2012). Vision and Agility Training in Community Dwelling Older Adults: Incorporating Visual Training into Programs for Fall Prevention. In: Gait \& Posture Vol. 35 (4): 585-589.

Röthig, P. \& Prohl, R. (2003). Sport (sport[s]). In: Röthig, P. \& Prohl, R. (Hrsg.) (2003). Sportwissenschaftliches Lexikon. 7., völlig neu bearb. Aufl., Schorndorf: 493 495.

Reicherts, M. \& Horn, A. B. (2009). Emotionen im Sport. In: Schlicht, W. \& Strauß, B. (Hrsg.) Grundlagen der Sportpsychologie. Göttingen: 563-633.

Rheinberg, F. $\left(2002^{4}\right)$. Motivation. Stuttgart.

Rheinberg, F., Vollmeyer, R. \& Engeser, S. (2003). Die Erfassung des FlowErlebens. In: Stiensmeier-Pelster, J. \& Rheinberg, F. (Hrsg.) (2003). Diagnostik von Motivation und Selbstkonzept. Göttingen: 261-279.

Rheinberg, F. (2006). Intrinsische Motivation und Flow-Erleben. In: Heckhausen, H. \& Heckhausen, J. (Hrsg.) (200633). Motivation und Handeln. Heidelberg: 331-354.

Rosenthal, G. (2005). Interpretative Sozialforschung. Eine Einführung. München.

Roth, K. \& Roth, C. (2009). Entwicklung koordinativer Fähigkeiten. In: Baur, J., Bös, K., Conzelmann, A. \& Singer, R. (Hrsg.) (2009²). Handbuch motorische Entwicklung. Schorndorf: 197-225.

Rott, C. (2008). Selbständigkeit in einer Gesellschaft des langen Lebens - die Rolle von körperlicher Aktivität. In: Mess, F., Dugandzic, D. \& Woll, A. (Hrsg.) (2008). Erfolgreiches Altern durch Sport. Konstanz: 9-33.

Schwenkmetzger, P. (2001). Psychologische Aspekte des Gesundheitssports. In: Gabler, H., Nitsch, J.R. \& Singer, R. (2001). Einführung in die Sportpsychologie, Teil 2: Anwendungsfelder. Schorndorf.

Ryan, R.M. \& Deci, E.L. (2007). Active Human Nature: Self-Determination Theory and the Promotion and Maintenance of Sport, Exercise and Health. In: Hagger, M.S. \& Chatzisarantis, N.L.D. (Hrsg.) (2007). Intrinsic Motivation and Self-Determination in Exercise and Sport. Leeds: 1-19.

Schaller, H.-J. \& Wernz, P. (2000). Koordinationstraining für Senioren. Aachen.

Schaller, H.-J. \& Wernz, P. $\left(2010^{3}\right)$. Koordinationstraining für Senioren. Besser orientieren, schneller reagieren, sicherer stehen und gehen. Aachen.

Schlicht, W. \& Schott, N. (2013). Körperlich aktiv altern. Weinheim \& Basel. 
Schmidtbleicher, D. (2009). Entwicklung der Kraft und Schnelligkeit. In: Baur, J., Bös, K., Conzelmann, A. \& Singer, R. (Hrsg.) (2009²). Handbuch motorische Entwicklung. Schorndorf: 149-166.

Schütze, F. (1977). Die Technik des narrativen Interviews in Interaktionsfeldstudien. Bielefeld.

Sebire, S.J., Standage, M. \& Vansteenkiste, M. (2009). Examining Intrinsic Versus Extrinsic Exersice Goals: Cognitive, Affective, and Behavioural Outcomes. In: Journal of Sport \& Exercise Psychology, Vol 31: 189-210.

Seidler, R.D. \& Schott, N. (2013). Mechanismen altersassoziativer Abnahmen im motorischen Lernprozess. In: Zeitschrift für Sportpsychologie, 20 (1): 18-24.

Singer, R. (Hrsg.) (1981). Alterssport. Versuch einer Bestandsaufnahme. Schorndorf.

Spirduso, W.W. (1995). Aging and motor control. In: Gisolfi, C., Lamb, D. \& Neal, E. (Hrsg.) (1995). Perspectives in exercise science and sports medicine: Exercise in older adults. Vol. 8: 53-110.

Staudinger, U. M. (2012). Fremd- und Selbstbild im Alter. Innen- und Außensicht und einige der Konsequenzen. In: Kielmansegg, P. G. \& Häfner, H. (Hrsg.) (2012). Alter und Altern. Wirklichkeiten und Deutungen. Heidelberg.

Stemper, T. (2001). Alter, Altern, Alterssport. Düsseldorf.

Stiggelbout, M., Hopman-Rock, M. \& van Mechelen, W. (2008). Entry Correlates and Motivations of Older Adults Participating in Organized exercise Programs. In: Journal of Aging and Physical Activity, Vol. 16: 342-354.

Stoll, O. Pfeffer, I. \& Alfermann, O. (2010). Lehrbuch Sportpsychologie. Bern.

Strübing, J. (2004). Grounded Theory. Zur sozialtheoretischen und epistemologischen Fundierung des Verfahrens der empirisch begründeten Theoriebildung. Wiesbaden.

Sudeck, G. (2007). Motivation und Volition in der Sport- und Bewegungstherapie. Hamburg.

Sudeck, G., Lehnert, K. \& Conzelmann, A. (2011). Motivbasierte Sporttypen. Auf dem Weg zur Personorientierung im zielgruppenspezifischen Freizeit- und Gesundheitssport. In: Zeitschrift für Sportpsychologie, 18 (1): 1-17.

Tang, A., Eng, J. J. \& Rand, D. (2012). Relationship between perceived and measured changes in walking after stroke. In: Journal of Neurology and Physical Therapy (JNPT). Vol. 30 (3): 119-21.

Tews, H. P. (2001). Aktivität und Altern in der alternden Gesellschaft. In: Daugs, R. et al. (Hrsg.) (2001). Aktivität und Altern. Schorndorf: 27-65. 
Tomasch, M. (1997). Seniorensport - ein Beitrag zum ,erfolgreichen' Altern. 1. Staatsprüfung, Universität Göttingen.

Vallerand, R.J. \& Loisier, G.F. (1999). An Integrative Analysis of Intrinsic and Extrinsic Motivation in Sport. In: Journal of Applied Sport Psychology 11: 142-169.

Vallerand et. al (2003). Les passions de l'âme: On obsessive and harmonious passion. In: Journal of Personality and Social Psychology, Vol. 85: 756-767.

Vansteenkiste, M. \& Deci, E.L. (2003). Competitively Contingent Rewards and Intrinsic Motivation: Can Losers Remain Motivated? In: Motivation and Emotion, Vol. 27/4: 273-300.

Vlachopoulos, S.P. \& Gigoudi, M.A. (2008). Why don't you exercise? Development of the Amotivation Toward Exercise Scale among older inactive individuals. In: Journal of Aging and Physical Activity, Vol. 16(3):316-41 .

Wagner, M. (2011). Motorische Leistungsfähigkeit im Kindes- und Jugendalter. Schorndorf.

Werle, J., Woll, A. \& Tittlbach, S. (2006). Gesundheitsförderung. Körperliche Aktivität und Leistungsfähigkeit im Alter. Stuttgart.

Willimczik, K. (2009). Sportmotorische Entwicklung. In: Schlicht, W. \& Strauß, B. (Hrsg.) Grundlagen der Sportpsychologie. Göttingen.

Willimczik, K. \& Singer, R. (2009). Motorische Entwicklung: Gegenstandsbereich. In: Baur, J., Bös, K., Conzelmann, A. \& Singer, R. (Hrsg.) (2009²). Handbuch motorische Entwicklung. Schorndorf: 15-24.

Wollesen, B. (2009). Gleichgewichts- und Koordinationstraining vs. Nordic Walking. Effekte zur Primärprävention und Sturzprophylaxe bei Senioren. Dissertation Universität Hamburg.

Wollny, R. (2002). Motorische Entwicklung in der Lebensspanne. Schorndorf.

Zarotis, G.F. (1999). Ziel Fitness-Club: Motive im Fitnesssport. Aachen.

\section{Online-Ressourcen:}

Göttinger Statistik Journal 4/2010 unter:

http://www.goesis.goettingen.de/pdf/journal/Journal2010-4.pdf

[Zugriff am 08.07.2013]

Deutscher Olympischer Sportbund (DOSB) unter:

http://www.dosb.de

[Zugriff am 19.05.2014] 
Deutscher Leichtathletikverband (DLV) unter:

http://www.leichtathletik.de

[Zugriff am 15.06.2014]

World Masters Athletics (WMA) unter:

http://www.world-masters-athletics.org

[Zugriff am 14.06.2014]

Deutscher Olympischer Sportbund (DOSB) unter:

http://www.deutsches-sportabzeichen.de

[Zugriff am 21.06.2014]

Self-Determination Theory (SDT) unter:

http://www.selfdeterminationtheory.org

[Zugriff am 03.08.2012]

Statistisches Bundesamt unter:

http://www.destatis.de

[Zugriff am 04.05.2014]

Bundespräsidialamt unter:

http://www.bundespraesident.de

[Zugriff am 14.04.2014]

Deutscher Olympischer Sportbund (DOSB) unter:

http://www.richtigfitab50.de

[Zugriff am 08.02.1014]

Süddeutsche Zeitung unter:

http://www.sueddeutsche.de/wirtschaft/rente-mit-union-und-spd-einigen-sich-imstreit-um-rentenpaket-1.1969098

[Zugriff am 28.05.2014]

Bestandserhebungen des Deutschen Olympischen Sportbundes (DOSB) unter: http://www.dosb.de/de/medien/downloads/statistiken/

[Zugriff am 05.07.2015] 


\section{Anhang}

Transkriptionsregeln (nach Rosenthal 2005: 94)

$\begin{array}{ll} & =\text { kurzes Absetzen, kleine Pause } \\ (3) & =\text { Dauer der Pause in Sekunden } \\ \mathrm{Ja}: & =\text { Dehnung eines Vokals } \\ ((\text { lacht })) & =\text { Reaktionen der Interviewten } \\ \text { /... } & =\text { Kommentar der Interviewerin } \\ \text { Nein } & =\text { betont } \\ \text { Viel- } & =\text { Abbruch des Wortes } \\ \text { (etwas }) & =\text { unsichere Transkription } \\ \text { Ja=ja } & =\text { schneller Anschluss }\end{array}$




\section{Eidesstattliche Erklärung}

Ich versichere, dass ich die eingereichte Dissertation "Motivation von Seniorinnen und Senioren zur sportlichen Betätigung: Eine empirische Untersuchung mittels qualitativer Interviews in und um Göttingen" selbstständig und ohne unerlaubte Hilfsmittel verfasst habe. Anderer als der von mir angegebenen Hilfsmittel und Schriften habe ich mich nicht bedient. Alle wörtlich oder sinngemäß den Schriften anderer Autoren entnommenen Stellen habe ich kenntlich gemacht.

Göttingen, d. 26.01.2015

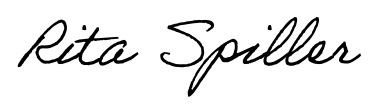

Rita Spiller 